\title{
DOSY External Calibration Curve Molecular Weight \\ Determination as a Valuable Methodology in Characterizing \\ Reactive Intermediates in Solution
}

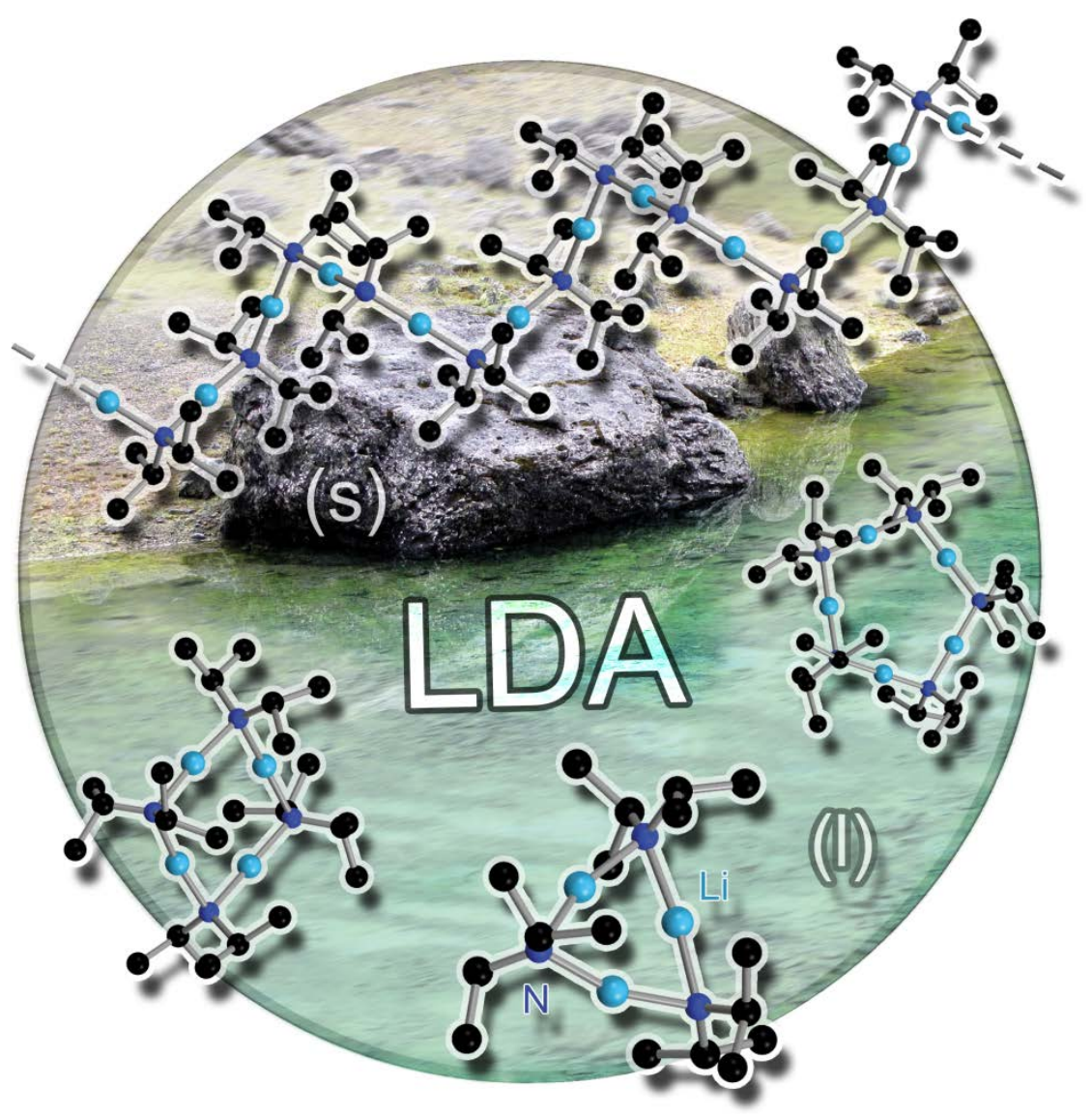

Frontispiece-Cover in Angewandte Chemie Int. Ed.

R. Neufeld, M. John, D. Stalke, Angew. Chem. Int. Ed., 2015, 54, 6994-6998.

The Donor-Base-Free Aggregation of Lithium Diisopropyl Amide in Hydrocarbons Revealed by a DOSY Method

Dissertation zur Erlangung des Doktorgrades

„Doctor rerum naturalium“

der Georg-August-Universität Göttingen

im Promotionsprogramm Chemie

der Georg-August University School of Science (GAUSS)

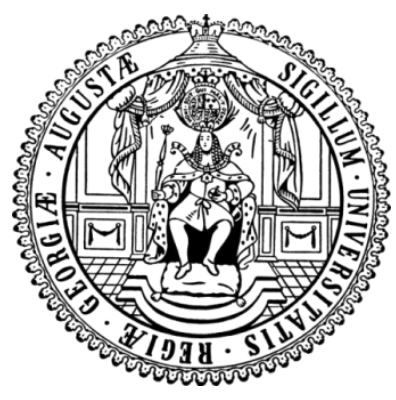

vorgelegt von Roman Neufeld aus Pawlodar 




\section{Betreuungsausschuss}

Prof. Dr. Dietmar Stalke, Institut für Anorganische Chemie

Prof. Dr. Sven Schneider, Institut für Anorganische Chemie

\section{Mitglieder der Prüfungskommission}

Referent: $\quad$ Prof. Dr. Dietmar Stalke

Korreferent: Prof. Dr. Sven Schneider

weitere Mitglieder der Prüfungskommision

Jun. -Prof. Selvan Demir, Institut für Anorganische Chemie

Prof. Ricardo A. Mata, Institut für Physikalische Chemie

Prof. Konrad Koszinowski, Institut für Organische und Biomolekulare Chemie

Dr. Michael John, Institut für Organische und Biomolekulare Chemie

Tag der mündlichen Prüfung: 14.03.2016 

... for my lovely family 

There must be a beginning of any great matter, but the continuing unto the end until it be thoroughly finished yields the true glory. 





\section{Table of Contents}

1 INTRODUCTION 1

1.1 Synthetically Useful Metal Amides $\quad 2$

1.1.1 Structure of LDA $\quad 6$

$\begin{array}{llr}\text { 1.1.1 Structure of LiTMP } & 7\end{array}$

1.1.2 Structure of LiHMDS and its Havier Analoges $\quad 9$

1.1.3 Structure of Hauser- and Turbo-Hauser Bases $\quad 12$

$\begin{array}{ll}1.2 & \text { Diffusion NMR Measurements }\end{array}$

1.2.1 Aspects of Diffusion $\quad 15$

1.2.2 The Hahn Spin-Echo Experiment 16

1.2.3 The Pulsed Gradient Spin-Echo (PFG-SE)-Experiment 17

1.2.4 The Pulsed Gradient Stimulated-Echo (PFG-STE)-Experiment 21

1.2.5 Advancements to the Stimulated-Echo 22

1.2.6 Extracting Diffusion Coefficients 23

$\begin{array}{ll}\text { 1.2.7 } & \text { Molecular Weight Determination } \\ \end{array}$

$\begin{array}{lll}1.3 & \text { Scope of this Thesis } & 30\end{array}$

$2 \quad$ RESULTS AND DISCUSSION 32

2.1 Development of the DOSY-External Calibration Curve MW-Determination Methodology 32

2.1.1 Application of a Normalized Reference System with Fixed Diffusion Coefficients 32

2.1.2 External Calibration Curves (ECC) and Internal References 35

2.1.3 Quality of TOL- $d_{7}$ and THF- $d_{7}$ as Internal Reference $\quad 39$

2.1.4 Influence of Shape $\quad 40$

$\begin{array}{ll}2.1 .5 & \text { Influence of Concentration }\end{array}$

2.1.6 Influence of Temperature 42

2.1.7 Influence of Halides and Molar Density 43

$\begin{array}{lll}\text { 2.1.8 Influence of Deuterated Compounds } & 47\end{array}$

2.2 DOSY-ECC-MW-Determination of Organometallics and Metal Amides 47

$\begin{array}{lll}\text { 2.2.1 Structure of LDA in THF Solution } & 47\end{array}$

2.2.2 Structure of LDA in Toluene Solution 48

2.2.3 Structure of Na-Indenide in THF Solution $\quad 52$

2.2.4 Structure of $[t-\mathrm{BuLi}]_{4} \cdot 4\left[\mathrm{Me}_{2} \mathrm{NC}_{6} \mathrm{H}_{4} \mathrm{Li}\right]_{4}$ in Toluene Solution 54

2.2.5 Structure of MHMDS with Ammonia as Donorbase in Toluene Solution 55

2.2.6 Structure of Hauser Base ${ }^{i} \operatorname{Pr}_{2} \mathrm{NMgCl}$ in THF solution 62

2.2.7 Structure of Turbo-Hauser Base ${ }^{i} \operatorname{Pr}_{2} \mathrm{NMgCl} \cdot \mathrm{LiCl}$ in THF solution $\quad 70$

2.2.8 Structure of Turbo-Hauser Base TMPMgCl-LiCl in THF solution 92

$3 \quad$ SUMMARY AND OUTLOOK $\quad 98$ 
$\begin{array}{ll}4.1 & 104\end{array}$

4.1.1 Handling of Air- and Moisture Sensitive Compounds 104

$\begin{array}{lll}\text { 4.1.2 NMR Experiments } & 104\end{array}$

$\begin{array}{ll}\text { 4.1.3 Computational Details } & 105\end{array}$

$\begin{array}{llr}4.2 & \text { Synthesis and Crystallization } & 106\end{array}$

$\begin{array}{lll}\text { 4.2.1 Donor-Base-Free LDA } & 106\end{array}$

$\begin{array}{ll}\text { 4.2.2 Na-Indenide } & 107\end{array}$

4.2.3 MHMDS-Ammoniacates $(\mathrm{M}=\mathrm{Li}, \mathrm{Na}, \mathrm{K}, \mathrm{Rb}$ and $\mathrm{Cs}) \quad 107$

$\begin{array}{ll}4.2 .4 \quad{ }^{i} \mathrm{Pr}_{2} \mathrm{NMgCl} & 108\end{array}$

$\begin{array}{lrr}4.2 .5 \quad{ }^{i} \mathrm{Pr}_{2} \mathrm{NMgCl} \cdot \mathrm{LiCl} & 108\end{array}$

$\begin{array}{lrr}\text { 4.2.6 TMPMgCl} \cdot \mathrm{LiCl} & 109\end{array}$

$\begin{array}{llr}4.3 & \text { Preparation of DOSY-NMR-Samples } & 110\end{array}$

$\begin{array}{llr}\text { 4.3.1 Internal References } & 110\end{array}$

$\begin{array}{lll}4.3 .2 & \text { LDA } & 110\end{array}$

$\begin{array}{ll}4.3 .3 \quad \text { Na-Indenide } & 110\end{array}$

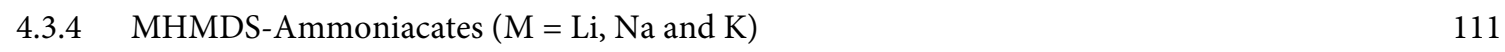

$\begin{array}{ll}\text { 4.3.5 }{ }^{i} \mathrm{Pr}_{2} \mathrm{NMgCl} \text { and }{ }^{\mathrm{i}} \operatorname{Pr}_{2} \mathrm{NMgCl} \cdot \mathrm{LiCl} & 111\end{array}$

$\begin{array}{lll}\text { 4.3.6 TMPMgCl} \cdot \mathrm{LiCl} & 111\end{array}$

$\begin{array}{llr}\text { 4.4-Ray Analysis } & 111\end{array}$

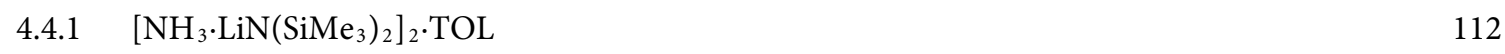

$\begin{array}{llr}4.4 .2 & {\left[\mathrm{NH}_{3} \cdot \mathrm{NaN}\left(\mathrm{SiMe}_{3}\right)_{2}\right]_{2}} & 113\end{array}$

$\begin{array}{llr}4.4 .3 \quad\left[\mathrm{NH}_{3} \cdot \mathrm{KN}\left(\mathrm{SiMe}_{3}\right)_{2}\right]_{2} & 114\end{array}$

$\begin{array}{llr}4.4 .4 \quad\left[\mathrm{NH}_{3} \cdot \mathrm{RbN}\left(\mathrm{SiMe}_{3}\right)_{2}\right]_{2} & 115\end{array}$

$\begin{array}{lll}4.4 .5 & \left(\mathrm{NH}_{3}\right)_{4} \cdot\left[\mathrm{NaN}\left(\mathrm{SiMe}_{3}\right)_{2}\right]_{2} & 116\end{array}$

$\begin{array}{lll}4.4 .6 \quad\left[(\mathrm{THF}) \mathrm{ClMg}\left(\mu-{ }^{i} \operatorname{Pr}_{2} \mathrm{~N}\right)\right]_{2} & 117\end{array}$

5 APPENDIX $r$

$\begin{array}{llr}6 & \text { REFERENCES } & 168\end{array}$

$\begin{array}{lr}\text { DANKSAGUNG } & 176\end{array}$

$\begin{array}{lr}\text { CURRICULUM VITAE } & 178\end{array}$ 


\section{Abbreviations}

\begin{tabular}{|c|c|}
\hline$c \mathrm{Hex}$ & cyclo-hexyl \\
\hline CIP & conact ion pair \\
\hline DMF & $N, N$-dimethylformamide \\
\hline DMSO & dimethyl sulfoxide \\
\hline DOSY & diffusion ordered spectroscopy \\
\hline Et & ethyl \\
\hline $\mathrm{Et}_{2} \mathrm{O}$ & diethyl ether \\
\hline HOESY & heteronuclear overhauser enhancement spectroscopy \\
\hline${ }^{i} \mathrm{Pr}$ & iso-propyle \\
\hline HMDS & 1,1,1,3,3,3-hexamethyldisilazane \\
\hline LDA & lithium diisopropylamide \\
\hline LiTMP & lithium 2,2,6,6-tetramethylpiperidide \\
\hline $\mathrm{Me}$ & methyl \\
\hline MHMDS & alkalimetal 1,1,1,3,3,3-hexamethyldisilazide \\
\hline${ }^{n} \mathrm{BuLi}$ & n-butyllithium \\
\hline NMR & nuclear magnetic resonance \\
\hline $\mathrm{Ph}$ & phenyl \\
\hline PMDETA & $N, N, N^{\prime}, N^{\prime \prime}, N^{\prime \prime}$-pentamethyldiethylenetriamine \\
\hline $\mathrm{RF}$ & radiofrequency \\
\hline SSIP & solvent-separated ion pair \\
\hline${ }^{t} \mathrm{BuLi}$ & tert-butyllithium \\
\hline $\sec$ & secondary \\
\hline TMP & 2,2,6,6-tetramethylpiperidide \\
\hline THF & tetrahydrofurane \\
\hline TOL & toluene \\
\hline TMCDA & $N, N, N, N$-tetramethylcyclohexane-1,2-diamine \\
\hline TMEDA & $N, N, N^{\prime}, N$ '-tetramethylethylene-1,2-diamine \\
\hline
\end{tabular}



Parts of this Ph. D. thesis have been published or submitted:

[1] R. Neufeld, R. Michel, R. Herbst-Irmer, R. Schöne, D. Stalke, "Introducing a Hydrogen Bond Donor Into a Non Nucleophilic Brønsted Base: Structural insights into Ammonia Coordinated Alkali Metal Hexamethyldisilazide (HMDS, Metal = Li, $\mathrm{Na}, \mathrm{K}, \mathrm{Rb}$ and Cs)", Chem. Eur. J. 2016, submitted.

[2] R. Neufeld, T. L. Teuteberg, R. Herbst-Irmer, R. A. Mata, D. Stalke, "Solution Structures of Hauser Base ${ }^{i} \mathrm{Pr}_{2} \mathrm{NMgCl}$ and Turbo-Hauser Base ${ }^{i} \mathrm{Pr}_{2} \mathrm{NMgCl} \cdot \mathrm{LiCl}$ in THF and the Influence of LiCl on the Schlenk-Equilibrium”, JACS 2016, accepted.

[3] R. Neufeld, D. Stalke, "Solution Structure of Turbo-Hauser Base TMPMgCl-LiCl in THF- $d_{8}$ ", Chem. Eur. J. 2016, submitted.

[4] S. Bachmann, R. Neufeld, M. Dzemski, D. Stalke, “New External Calibration Curves (ECCs) for the Determination of Molecular Weights in Various Common NMR Solvents", Chem. Eur. J. 2016, accepted.

[5] R. Neufeld, M. John, D. Stalke, “The Donor-Base-Free Aggregation of Lithium Diisopropyl Amide in Hydrocarbons Revealed by a DOSY Method", Angew. Chem. 2015, 127, 7100-7104; Angew. Chem. Int. Ed. 2015, 54, 6994-6998.

[6] R. Neufeld, D. Stalke, “Accurate Molecular Weight Determination of Small Molecules via DOSY-NMR by Using External Calibration Curves with Normalized Diffusion Coefficients", Chem. Sci. 2015, 6, 3354-3364.

[7] R. Michel, T. Nack, R. Neufeld, J. M. Dieterich, R. A. Mata, D. Stalke, “The Layered Structure of $\left[\mathrm{Na}\left(\mathrm{NH}_{3}\right)_{4}\right]$ [Indenide] Containing a Square-Planar $\mathrm{Na}\left(\mathrm{NH}_{3}\right)_{4}{ }^{+}$Cation", Angew. Chem. 2013, 125, 762-766; Angew. Chem. Int. Ed. 2013, 52, 734-738. 


\section{INTRODUCTION}

Besides synthetically useful organolithium compounds ${ }^{[8]}$ like MeLi, ${ }^{n} \mathrm{BuLi},{ }^{t} \mathrm{BuLi}$ und $\mathrm{PhLi}$, especially secondary organoamides $\left(\mathrm{RR}^{\prime} \mathrm{N}^{-}\right)$play a huge role in organic chemistry. In contrast to organolithiums which may be used as nucleophiles in useful addition or substitution reactions, ${ }^{[9]}$ sterically hindered $s e c$-organoamides are predominantly used as powerful non nucleophilic Brønsted bases in synthetic chemistry. ${ }^{[10]}$ A variety of amidolithium compounds are commercially available. However, their chemical formula is often depicted as monomeric species, although it has been known for a long time that the actual structure is much more complicated. The aggregation of sec-amidolithium compounds differs in respect to their substituents. Studies in solution as well as in the solid state reveal several aggregation modes varying from monomers, cyclic dimers, trimers, tetramers and higher oligomers (see next chapter), furthermore dimeric units can associate laterally to form "ladder" like coordination polymers. ${ }^{[1]}$ Usually, disaggregated amidolithiums show similar to organolithiums an enhanced reactivity and a different chemoselectivity (Scheme 1-1). ${ }^{[12]}$ This is why the knowledge of the structure in the solid state and especially in solution where they operate is extraordinary important to deduce structure-reactivity relationships. ${ }^{[13]}$ In the following chapters a brief synthetical and structural overview of the most important sec-organoamides will be given. Finally, the use of self-diffusion measurements as a valuable tool for the determination of aggregation states will be introduced.

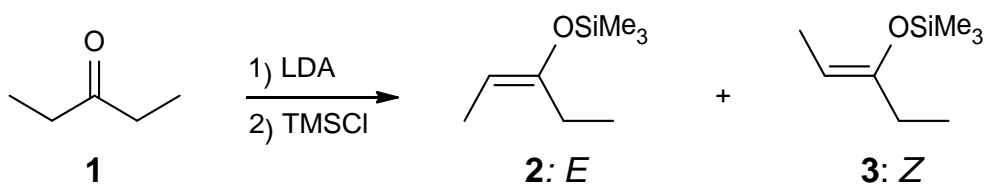

\begin{tabular}{cccc}
\hline Solvent & E/Z ratio & Yield [\%] & Lit. \\
\hline Hexane & $1.0: 1.5$ & 14 & {$[12 \mathrm{a}]$} \\
THF & $0.5: 99.5$ & quant. & {$[12 \mathrm{~b}]$}
\end{tabular}

Scheme 1-1. Enolization of 3-pentanone 1 with lithium diisopropylamide (LDA) in hexane and subsequent trapping with TMSCl affords silylenolates 2 and 3 in a $E / Z$ ratio of 1.0:1.5 in very poor yields $(14 \%) .{ }^{[2 a]}$ In contrast, the same reaction carried out in THF gives highly stereoselectively the (Z)-3-trimethylsiloxy-2pentane 3 in quantitative yield. ${ }^{[12 b]}$ The change in reactivity and stereoselectivity is reflected in the different aggregation states of LDA in both solvents. In hexane LDA forms a distribution of at least three cyclic oligomers, ${ }^{[5]}$ while in THF it forms exclusively a disolvated dimer ${ }^{[14]}$ (for more information about the solution structure of LDA see chapter 1.1.1 and 2.2.2). 


\subsection{Synthetically Useful Metal Amides}

The most important secondary amides used in synthetic chemistry are LDA 4, LiTMP 5, MHMDS 6 with $\mathrm{M}=\mathrm{Li}, \mathrm{Na}$ and $\mathrm{K}$, Hauser bases 7 and 8 and their lithium chloride stabilized Turbo- analogues 9 and 10 (Fig. 1-1).<smiles>CC(C)N([AlH])C(C)C</smiles>

4<smiles>CC1(C)CCCC(C)(C)N1[AlH2]</smiles>

5<smiles>C[Si](C)(C)N([AlH])[Si](C)(C)C</smiles>

6

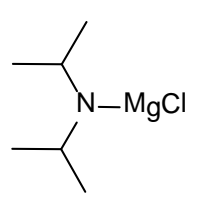

7

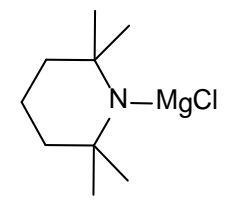

8

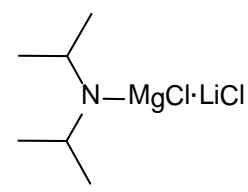

9

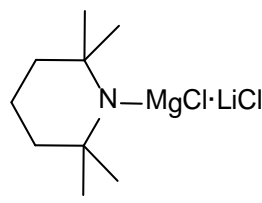

10

Fig. 1-1. Some of most important secondary amides in synthetic chemistry.

Commonly, alkalimetal amides are prepared by treating the secondary amine $\mathrm{R}_{2} \mathrm{NH}$ with an alkyl lithium reagent (e.g. $\left.{ }^{n} \mathrm{BuLi}\right)$ in a cooled THF solution $\left(-78^{\circ} \mathrm{C}\right)$. Diisopropylamide has a $\mathrm{p} K_{\mathrm{a}}$ value of $36 .{ }^{[15]}$ Its conjugate base is therefore suitable for the deprotonation of compounds with lower acidity.

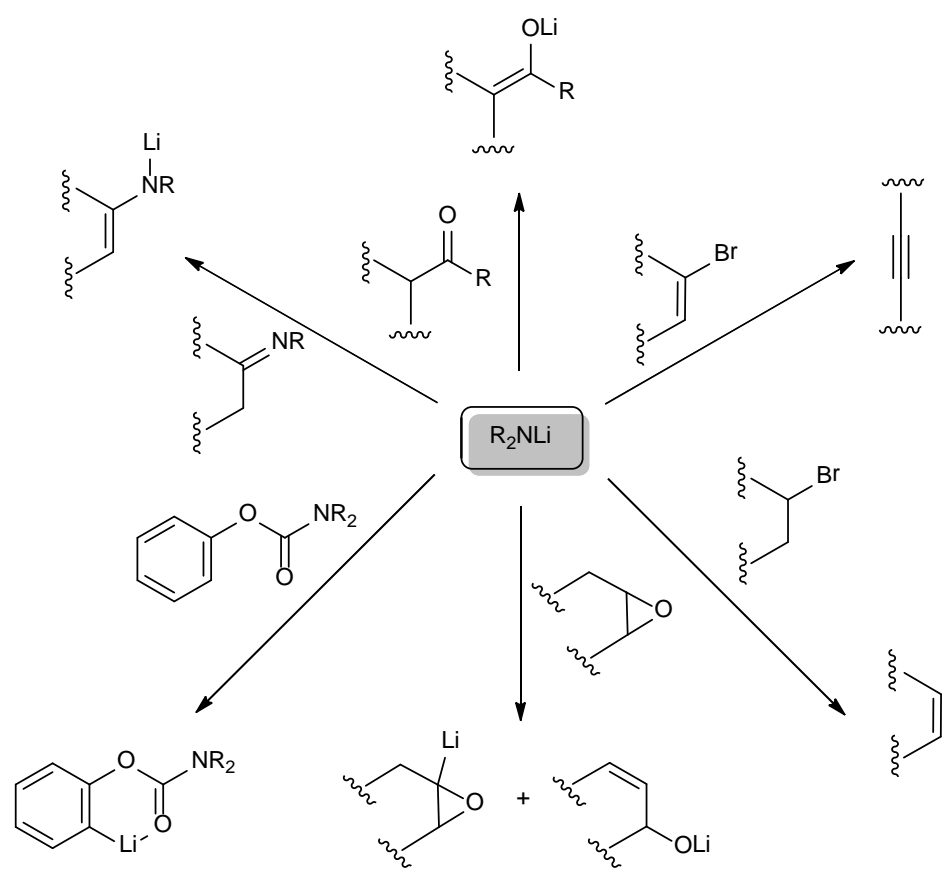

Scheme 1-2. Prominent reactions of lithium amides. ${ }^{[13 c]}$ 
Due to the steric demand of the amide ligand alkalimetal amides represent excellent reagents for the generation of enolates by abstraction of the acidic a-hydrogen atom of a carbonyl function. The most prominent reactions of lithium amides are summarized in Scheme 1-2. The high reactivity of organolithium compounds can also be considered their Achilles heel, since they are routinely used at low temperature (mostly $-78^{\circ} \mathrm{C}$ ). Additionally they often cause competing side reactions (e.g. Chichibabin reactions) and do not tolerate certain synthetically important functional groups like e.g. ester, carbonyl, nitrile, sulphoxide and halide. This is why in the past decades bis-amidomagnesium bases $\left[\left(\mathrm{R}_{2} \mathrm{~N}\right) \mathrm{Mg}\right]$ and organomagnesium halides (at the simplest level described with the formula "RMgX") have found profund improvements in synthetic chemistry. Compared with organolithium reagents the magnesium compounds have more covalent character and therefore less reactive metal-ligand bonds. This is why they display a higher functional group tolerance and a much greater chemoselectivity. ${ }^{[16]}$ Consequently they generally can be used at room temperature (RT) without significant side reactions. In the late $1940 \mathrm{~s}$ Hauser and co-workers succeeded in the development of the amido Grignard reagent $\mathrm{R}_{2} \mathrm{NMgX}$, formally known as Hauser bases, by replacing the alkyl ligand of a Grignard with a secondary amide (Scheme 1-3). ${ }^{[17]}$

$$
\mathrm{RMgCl}+\mathrm{R}_{2} \mathrm{NH} \underset{\mathrm{RT}}{\stackrel{\mathrm{THF}}{\longrightarrow}} \mathrm{R}_{2} \mathrm{NMgCl}+\mathrm{RH}
$$

Scheme 1-3. Typical preparation of Hauser bases.

The breakthrough in synthetic protocols of Hauser bases culminates in the 1980s and 1990s, where Eaton and co-workers introduced ${ }^{i} \operatorname{Pr}_{2} \mathrm{NMgBr}$ and $\mathrm{TMP}_{2} \mathrm{Mg}$, which were shown to ortho-magnesiate carboxamides (Scheme 1-4a). ${ }^{[18]}$ Later, Kondo, Sakamoto and co-workers reported the utility of ${ }^{i} \operatorname{Pr}_{2} \mathrm{NMgX}(\mathrm{X}=\mathrm{Cl}, \mathrm{Br})$ as selective deprotonation reagents (exclusively at the 2-position) for heterocyclic thiophene ${ }^{[19]}$ and phenylsulphonylsubstituted indoles ${ }^{[20]}$ (Scheme 1-4b-c). An important driving force for such reactions is the presence of an ortho-directing group on the substrate, typically Lewis basic heteroatoms within or next to a double or triple bond. The principle of the functional group is known as "Direct ortho Metallation" (DoM) and has been elaborated by Snieckus and Beak. ${ }^{[21]}$ 
a)

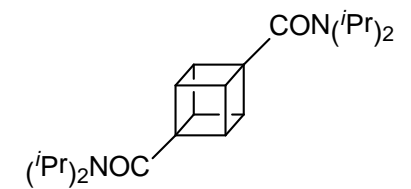

11

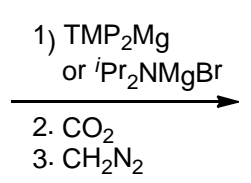

3. $\mathrm{CH}_{2} \mathrm{~N}_{2}$<smiles>COC(=O)c1cc(C(=O)O[Na])ccc1C(=O)ONc1ccccc1</smiles>

12: $80 \%$

b)

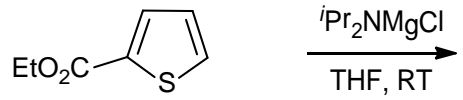

13

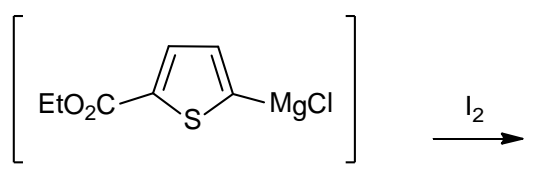

14<smiles>CCOC(=O)c1ccc(I)s1</smiles>

15: $77 \%$

c)<smiles></smiles>

16

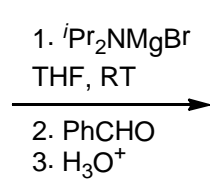

3. $\mathrm{H}_{3} \mathrm{O}$<smiles>O=S(=O)(c1ccccc1)c1cc2ccccc2n1-c1ccccc1</smiles>

17: $83 \%$

Scheme 1-4. a) Compound 12 can be synthesized by a-magnesiation of carboxamide 11 with $\mathrm{TMP}_{2} \mathrm{Mg}$ or ${ }^{i} \mathrm{Pr}_{2} \mathrm{NMgBr}$ followed by the subsequent reaction with $\mathrm{CO}_{2}$ and $\mathrm{CH}_{2} \mathrm{~N}_{2} \cdot{ }^{[18]}$ b) 2-carbethoxythiophene 13 was metalated at the ortho position by using an excess of ${ }^{i} \operatorname{Pr}_{2} \mathrm{NMgCl}$ ( 2 equiv). Afterwards the magnesiated intermediate 14 was treated with iodine to give iodothiophene 15 in $77 \%$ yield. c) Selective magnesiation of indole 16 in ortho position followed by the reaction with benzaldehyde give the substituted indole 17. ${ }^{[20]}$

$$
{ }^{i} \mathrm{PrMgCl}_{\mathrm{LiCl}} \underset{\mathrm{THF}}{\stackrel{\mathrm{R}_{2} \mathrm{NH}}{\longrightarrow}} \mathrm{R}_{2} \mathrm{NMgCl} \cdot \mathrm{LiCl} \underset{\mathrm{THF}}{\stackrel{\mathrm{MgCl}_{2}}{\stackrel{\mathrm{T}}{\mathrm{T}}}} \mathrm{R}_{2} \mathrm{NLi}
$$

Scheme 1-5. Typical preparation of Turbo-Hauser bases.

A huge disadvantage of Hauser bases is their poor solubility in THF. In consequence, the metalation rates are slow and a huge excess of base is required (mostly 10 equiv.). This circumstance complicates the functionalization of the metaled intermediate with an electrophile. It is well known that numerous metallic salts are better soluble when $\mathrm{LiCl}$ is added to the solution. ${ }^{[22]}$ That feature led to the design of LiCl-solubilized Turbo-Hauser Bases TMPMgCl.LiCl 10 and the less bulky Turbo- analogue ${ }^{i} \operatorname{Pr}_{2} \mathrm{NMgCl} \cdot \mathrm{LiCl} 9$ (Scheme 15). ${ }^{[23]}$ Equipped with enhanced kinetic basicity, ${ }^{1}$ these commercially available reagents

\footnotetext{
${ }^{1}$ For the sake of completeness it should be mentioned that in modern synthetic chemistry many other lithium chloride stabilized magnesium amide complexes like e.g. (TMP) ${ }_{2} \mathrm{Mg} \cdot 2 \mathrm{LiCl}, \mathrm{TMPZnCl} \cdot \mathrm{LiCl}(\mathrm{TMP})_{2} \mathrm{Zn} \cdot 2 \mathrm{LiCl}$ or mixed metal ate complexes like e.g. (TMP) ${ }_{2} \mathrm{CuLi}, \mathrm{R}_{2} \mathrm{Zn}(\mathrm{TMP}) \mathrm{Li}, \mathrm{R}_{3} \mathrm{Al}(\mathrm{TMP}) \mathrm{Li}$ are also used as selective deprotonating reagents. For more information see e.g.: a) B. Haag, M. Mosrin, H. Ila, V. Malakhov, P. Knochel, Angew. Chem. 2011, 123, 9968-9999; Angew. Chem. Int. Ed. 2011, 50, 9794-9824. b) R. E. Mulvey, F. Mongin, M. Uchiyama, Y. Kondo, Angew. Chem. 2007, 119, 3876-3899; Angew. Chem. Int. Ed. 2007, 46, 3802-3824.
} 
display a high reactivity even at room temperature (RT) as well as excellent regioselectivity and high functional group tolerance for a large number of aromatic and heteroaromatic substrates (Scheme 1-6). ${ }^{[24]}$

a)<smiles>CCOC(=O)c1cccc(Cl)c1C#N</smiles>

18

$$
\begin{aligned}
& \text { 1) } \mathrm{TMPMgCl} \cdot \mathrm{LiCl} \\
& \mathrm{TH} \mathrm{TH} / \mathrm{Et}_{2} \mathrm{O}(1: 2) \\
& -20^{\circ} \mathrm{C}, 5 \mathrm{~h} \\
& \hline \text { 2) } \mathrm{EtOCOCN} \\
& -40 \text { to } 25^{\circ} \mathrm{C}, 1 \mathrm{~h}
\end{aligned}
$$

b)<smiles>CCOC=CC(=O)OCC</smiles>

$$
\underset{\mathrm{THF},-15^{\circ} \mathrm{C}, 1 \mathrm{~h}}{\stackrel{\text { TMPMgCl} \cdot \mathrm{LiCl}}{\longrightarrow}}
$$

20

c)<smiles>CCOC(=O)c1ccc(Cl)c(C(=O)OCC)c1C#N</smiles>

19: $60 \%$

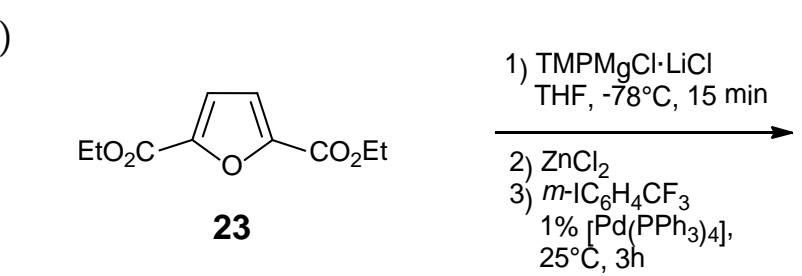
23

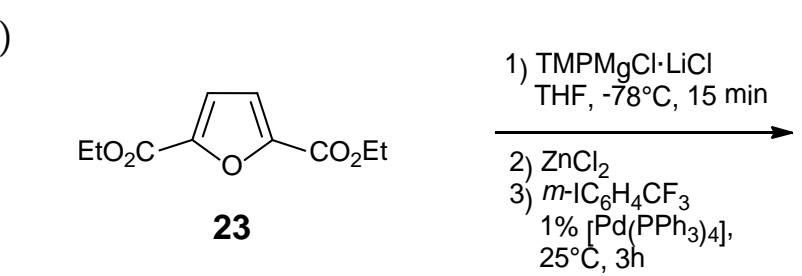

$25^{\circ} \mathrm{C}, 3 \mathrm{~h}$

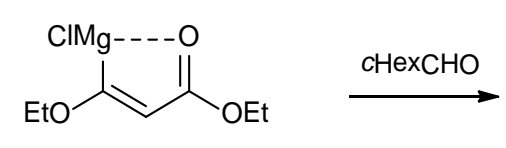

21

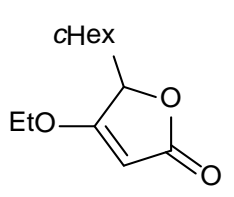

22: $85 \%$

Scheme 1-6. a) Metalation of ethyl 2-cyano,3-chlorobenzoate 18 with $\mathrm{TMPMgCl} \cdot \mathrm{LiCl}(\mathbf{1 0})$ and reaction with EtOCOCN gives the benzene derivative 19 in $60 \%$ yield. ${ }^{[25]}$ b) Compound 10 allows also selective deprotonations of 1,4 unsaturated compounds like e.g. the Michael acceptor 20. The addition of compound 21 to $\mathrm{cHexCHO}$ affords the unsaturated lactone $\mathbf{2 2}$ in $85 \%$ yield. ${ }^{[26]} \mathrm{c}$ ) Metalation of furane $\mathbf{2 3}$ and subsequent transmetalation with $\mathrm{ZnCl}_{2}$ leads to a stable $\mathrm{Zn}$-intermediate that can in presence of $1 \%\left[\mathrm{Pd}\left(\mathrm{PPh}_{3}\right)_{4}\right]$ undergo a cross-coupling with an aryl iodide to give the functionalized furane $24 .{ }^{[27]}$

Interestingly, the diisopropylamido reagent ${ }^{i} \operatorname{Pr}_{2} \mathrm{NMgCl} \cdot \mathrm{LiCl} 9$ can show a very different reactivity compared to its TMP counterpart 10 (Scheme 1-7). While 10 easily metalates ethyl-3-chlorobenzoate $\mathbf{2 6}$ in the C2 position to give after iodation benzene $\mathbf{2 7}$, the same reaction carried out with 9 results in no metalation at all. Instead, an addition-elimination reaction occurs with the formation of $m$-chloro-N,N-diisopropylbenzamide $25 .{ }^{[28]}$ The different reactivity can be attributed to the higher kinetic basicity of compound $\mathbf{1 0}$ compared to its homologous ${ }^{i} \operatorname{Pr}$-Turbo-Hauser base 9. ${ }^{[23]}$ Additionally, this contrasting behaviour could also be reflected in the aggregation state of both magnesium amides in THF solution that will be discussed in chapter 1.1.3. 
<smiles>O=C(Nc1ccccc1)c1cccc(Cl)c1</smiles>

25: $91 \%$
1) ${ }^{i} \mathrm{Pr}_{2} \mathrm{NMgCl} \cdot \mathrm{LiCl}$ $\mathrm{THF}, 0^{\circ} \mathrm{C}$<smiles>CCOC(=O)c1cccc(Cl)c1</smiles>

26

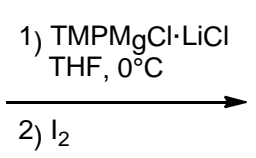

TMPMgCl$\cdot \mathrm{LiCl}$
$\mathrm{THF}, 0^{\circ} \mathrm{C}$
$\mathrm{I}_{2}$<smiles>CCOC(=O)c1cccc(Cl)c1I</smiles>

27: $74 \%$

addition-elimination

Scheme 1-7. Contrasting reactivity of Turbo-Hauser bases ${ }^{i} \mathrm{Pr}_{2} \mathrm{NMgCl} \cdot \mathrm{LiCl} 9$ and TMPMgCl$\cdot \mathrm{LiCl} 10$ for the reaction with ethyl-3-chlorobenzoate 26 in THF solution at $0^{\circ} \mathrm{C} .{ }^{[28]}$

\subsubsection{Structure of LDA ${ }^{2}$}

LDA is one of the most prominent amide reagents that play a key role in organic synthesis, serving as a base par excellence for a broad range of deprotonation reactions. ${ }^{[29]}$ However, its donor-base free solid-state crystal structure 28 was only determined in 1991 by Barnett et al. ${ }^{[30]}$ It consists of an infinite helical chain with four units per turn in the helix (Fig. 1-2). In solution with monodentate donating solvents LDA exists as a single observable aggregate-the disolvated cyclodimer 29. ${ }^{[14]}$ That $\mathrm{Li}_{2} \mathrm{~N}_{2}$ dimer with one donor molecule coordinating each alkali metal atom, afford an overall lithium coordination number of three. Polydentate ligands give isostructural disolvated dimers with one exception: the TMCDA-solvated monomer 30. This is why LDA is an ideal template for studying organolithium reactivity ${ }^{[13 c, 31]}$ and $\mathrm{LDA}$ is one of the best explored lithium amides. ${ }^{[10]}$ Especially Collum et al. provided closer insights into LDA-mediated reaction mechanisms, solution kinetics, structure reactivity relationships, reaction rates and selectivity. ${ }^{[12 a, 32]}$ However, the aggregation of LDA in donor-base free solvents was still unclear. In $1991 \mathrm{Kim}$ and Collum et al. investigated $\left[{ }^{6} \mathrm{Li}\right] \mathrm{LDA}$ and $\left[{ }^{6} \mathrm{Li},{ }^{15} \mathrm{~N}\right] \mathrm{LDA}$ in hexane by ${ }^{6} \mathrm{Li}$ and ${ }^{15} \mathrm{~N}$ NMR spectroscopy. ${ }^{[12 a]}$ They observed a mixture of three major cyclic oligomers and suggested that they could correspond to cyclic dimers, trimers and higher oligomers. Unfortunately they were not able to quantify these observations because "a severe overlap renders the effort required for a detailed study unjustifiable". A further characterization of these oligomers was not possible at that time.

\footnotetext{
${ }^{2}$ Includes revised parts of my publication: R. Neufeld, M. John, D. Stalke, Angew. Chem., 2015, 127, 71007104; Angew. Chem. Int. Ed., 2015, 54, 6994-6998.
} 


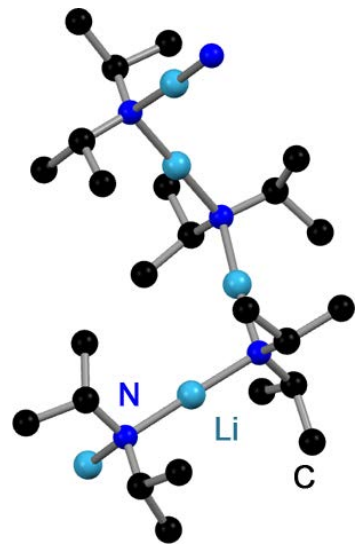

28<smiles>CC(C)N1[SiH](S)N(C(C)C)[Si]1(C(C)C)C(C)(C)C</smiles>

29a $S=T H F$

29b $\mathrm{S}=\mathrm{R}_{2} \mathrm{O}$

29c $S={ }^{n}$ BuOMe

29d S $=\mathrm{Me}_{2} \mathrm{NEt}$

29e $S=d m p u$

29f $S=h m p a$

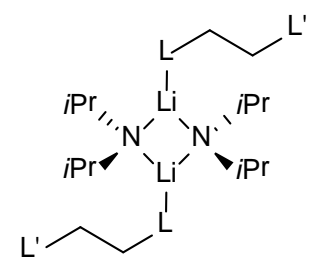<smiles></smiles>

30

Fig. 1-2. Solid state structures $(\mathbf{2 8})^{[30]}$ and solution structures $(\mathbf{2 9}, \mathbf{3 0})$ of LDA. ${ }^{[13 c]}$

\subsubsection{Structure of LiTMP}

In 1983 Lappert and Atwood et al. revealed the solid state structure of unsolvated lithium 2,2,6,6-tetramethylpiperidide to be a cyclotetramer 32 (Fig. 1-3) with an essentially planar $\mathrm{Li}_{4} \mathrm{~N}_{4}$-ring. ${ }^{[33]}$ Later, ${ }^{6} \mathrm{Li},{ }^{15} \mathrm{~N}$ NMR and DOSY spectroscopic studies showed that in hydrocarbon solution cycloteramer and -trimer co-exist in a balanced equilibrium. ${ }^{[34]}$ Three decades after Lapperts report, Hevia and Mulvey et al. finally characterized the concealed cyclotrimeric polymorph 31 in the solid state via X-ray crystallography. ${ }^{[34 a]}$ In donating solvents LiTMP shows a much greater structural diversity. Beside the above mentioned cyclic oligomers LiTMP can form disolvated monomers 33 and $34,{ }^{[35]} \mathrm{di}$ - and tetrasolvated dimers 35 and 36, ${ }^{[36]}$ triple ions $37^{[37]}$ and open dimers 38 and 39 (Fig. 1-4). ${ }^{[38]}$ The latter have shown to be important reactive intermediates since they contain vacant coordination sites for substrate precomplexation and a highly basic lone pair on the nitrogen atom.

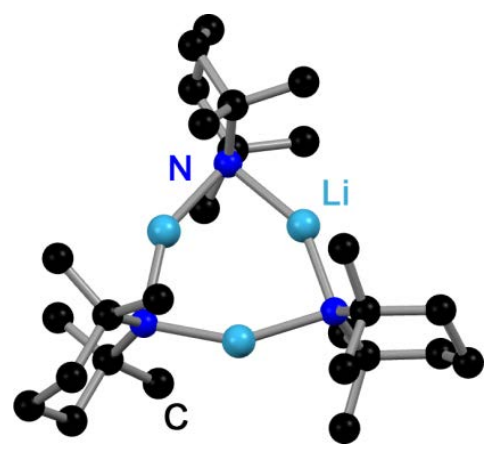

31

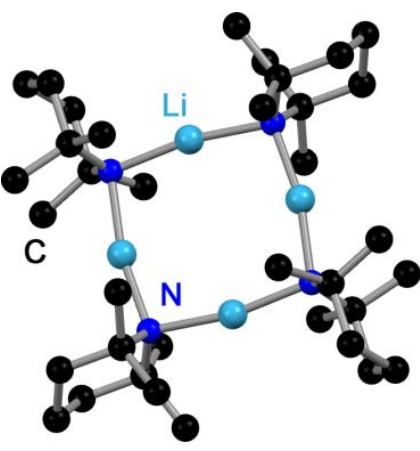

32

Fig. 1-3. Structure of LiTMP in the solid state and in hydrocarbons with hydrogen atoms omitted for clarity. 

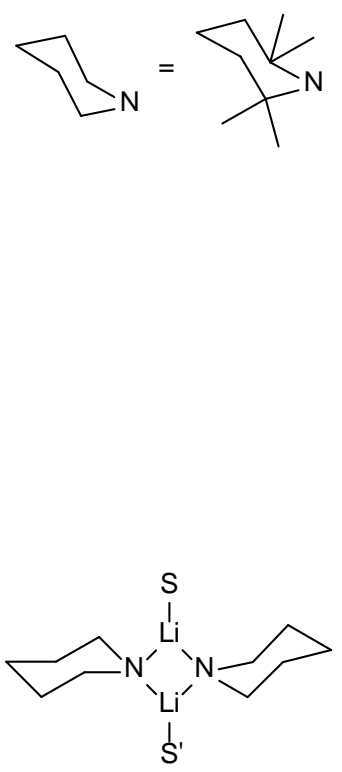

35a $\mathrm{S}=\mathrm{S}^{\prime}=\mathrm{THF}$

35b $S=S^{\prime}=$ HMPA

35c $S=$ THF,$S^{\prime}=$ HMPA

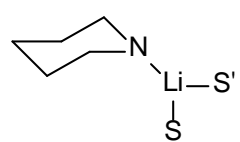

33a $\mathrm{S}=\mathrm{S}^{\prime}=\mathrm{THF}$

33b $\mathrm{S}=\mathrm{S}^{\prime}=\mathrm{HMPA}$

33c $\mathrm{S}=\mathrm{THF}, \mathrm{S}^{\prime}=\mathrm{HMPA}$

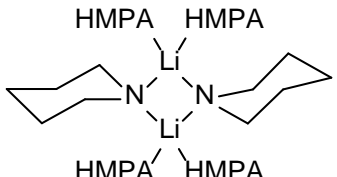

36

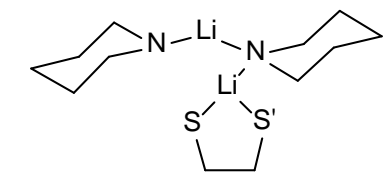

38a $\mathrm{S}=\mathrm{S}^{\prime}=\mathrm{NMe}_{2}$

38b $\mathrm{S}=\mathrm{S}^{\prime}=\mathrm{NEt}_{2}$

38b $\mathrm{S}=\mathrm{S}^{\prime}=\mathrm{N}(\mathrm{Me}) \mathrm{Et}$

38c $\mathrm{S}=\mathrm{S}^{\prime}=\mathrm{N}\left(\mathrm{C}_{4} \mathrm{H}_{8}\right)$

38d $\mathrm{S}=\mathrm{NMe}_{2}, \mathrm{~S}^{\prime}=\mathrm{N}(\mathrm{Me}) \mathrm{Et}$

38e $\mathrm{S}=\mathrm{NMe}_{2}, \mathrm{~S}^{\prime}=\mathrm{OMe}$

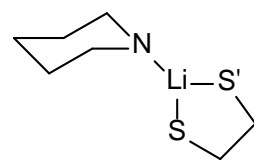

34a $\mathrm{S}=\mathrm{S}^{\prime}=\mathrm{NMe}_{2}$

34b $\mathrm{S}=\mathrm{S}^{\prime}=\mathrm{NEt}_{2}$

34b $\mathrm{S}=\mathrm{S}^{\prime}=\mathrm{N}(\mathrm{Me}) \mathrm{Et}$

34c $\mathrm{S}=\mathrm{S}^{\prime}=\mathrm{N}\left(\mathrm{C}_{4} \mathrm{H}_{8}\right)$

34d $\mathrm{S}=\mathrm{NMe}_{2}, \mathrm{~S}^{\prime}=\mathrm{N}(\mathrm{Me}) \mathrm{Et}$

$34 \mathrm{e} S=\mathrm{NMe}_{2}, \mathrm{~S}^{\prime}=\mathrm{OMe}$

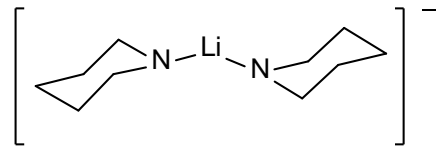

37a $+\left[\mathrm{Li}(\mathrm{HMPA})_{3}(\mathrm{THF})\right]^{+}$

37b $+\left[\mathrm{Li}(\mathrm{HMPA})_{4}\right]^{+}$

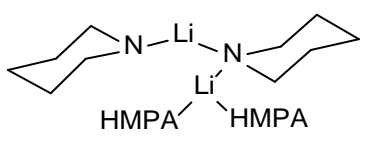

39

Fig. 1-4. Structural diversity of LiTMP in mono- and polydentate solvents.

The equilibrium between monomeric $\mathbf{3 4}$ and open dimeric $\mathbf{3 8}$ highly depends on the used chelating ligand and its concentration. E.g. the ratio of $\mathbf{3 4 \mathbf { d }}$ and $\mathbf{3 8 d}$ is $1: 3$, while the ratio is completely inverted for species $34 \mathbf{e}$ and $38 \mathrm{e}$ ( 0.75 eq. of ligand, at $-100^{\circ} \mathrm{C}$ in a $2: 1$ pentane:toluene solution). ${ }^{[38 a]}$ Most of the solution studies have been carried out on the conformationally locked but isostructural 2,2,4,6,6-pentamethylpiperidide (LiPMP). ${ }^{[38 a]}$ 


\subsubsection{Structure of LiHMDS and its Havier Analoges ${ }^{3}$}

Besides LDA and LiTMP alkali metal 1,1,1,3,3,3-hexamethyldisilazides (MHMDSs), particularly $\mathrm{Li}^{-}$, $\mathrm{Na}-$ and $\mathrm{KHMDS}$ are very important reagents in many synthetic protocols. ${ }^{[10]}$ The high structural variance of MHMDS becomes apparent from the different aggregation motifs in the solid state, in the gas phase and in solution: The solid state structure of $\mathrm{Li}^{\left[{ }^{[39]}\right.}$ and $\mathrm{NaHMDS}^{[40]}$ is a cyclic trimer (40 in Fig. 1-5), whereas the latter also crystallizes as a linear coordination polymer $(42) \cdot{ }^{[41]} \mathrm{K}-{ }^{[42]} \mathrm{Rb}$ - and CsHMDS form dimeric structures (44, without donor base). ${ }^{[43]}$ In the gas phase LiHMDS adopts dimers ${ }^{[44]}(\mathbf{4 4}$, without donor base) and NaHMDS monomers ${ }^{[45]}$ (43, without donor base). The adducts of $\mathrm{Li}_{-},{ }^{[46]} \mathrm{Na}^{-[47]}$ and KHMDS ${ }^{[42 b,}{ }^{48]}$ with monodentate donor bases show dimeric (44) and polymeric structures (polymers of $\mathbf{4 4}$, bridged by donor bases). Monomeric adducts (43) of $\mathrm{Li}^{-[49]}$ and ${ }^{[H M D S}{ }^{[50]}$ have been observed so far only with chelating bases like TMEDA and crown ethers. $\mathrm{Rb}$ - and CsHMDS were characterized as coordination polymers of dimers bridged by dioxane. ${ }^{[47 \mathrm{~d}]}$ In solution LiHMDS shows a complex equilibrium of solvation and aggregation depending on the employed solvent. Kimura and Brown reported in 1971 that LiHMDS exists as a tetramer-dimer mixture in hydrocarbons and as dimer-monomer mixture in THF and $\mathrm{Et}_{2} \mathrm{O} \cdot{ }^{[51]}$ Later, especially Collum and Lucht carried out deeply-rooted mechanistic studies to elucidate the aggregation of LiHMDS with various amine and ether ligands by ${ }^{6} \mathrm{Li},{ }^{15} \mathrm{~N}$, and ${ }^{13} \mathrm{C}$ NMR spectroscopic studies. ${ }^{[52]}$ Williard and Mulvey et al. studied and characterized mixed $\mathrm{Li}^{-}, \mathrm{Na}-$ and KHMDS bases in the solid state and in solution. ${ }^{[46 a, 46 c, 53]}$ Compared to the wide synhetic application, both on laboratory and industrial scale, there still is not known much about the aggregation behavior of higher MHMDSs in solution.

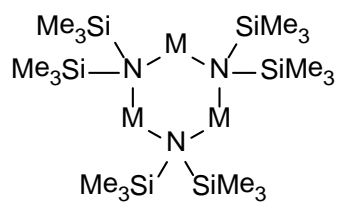

40

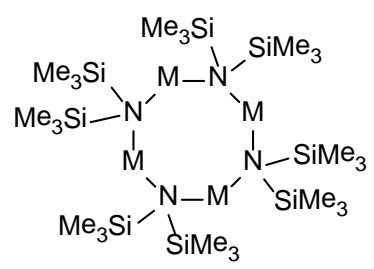

41

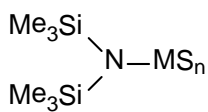

43

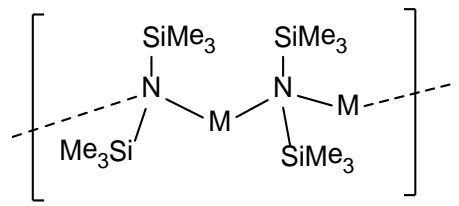

42

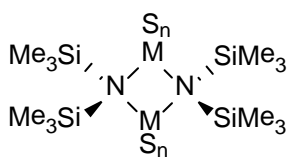

44

Fig. 1-5. Aggregation modes of MHMDS. S = donor base like e.g. THF with $\mathrm{n}=1-3$.

\footnotetext{
${ }^{3}$ Includes revised parts of my publication: R. Neufeld, R. Michel, R. Herbst-Irmer, R. Schöne, D. Stalke, 2016, submitted.
} 


\subsubsection{Structure of MHMDS with Ammonia as Donor Base ${ }^{4}$}

a)

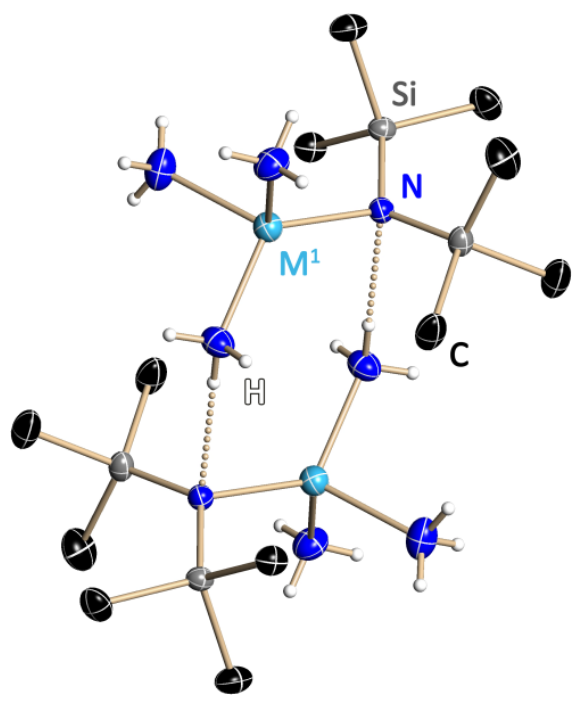

$$
\begin{aligned}
45 \mathrm{M}^{1} & =\mathrm{Li} \\
46 \mathrm{M}^{1} & =\mathrm{Na}
\end{aligned}
$$

c)

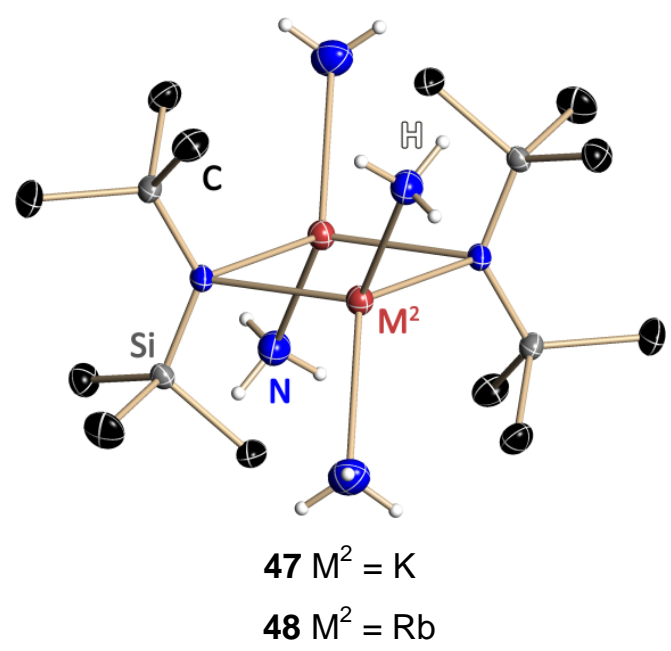

b)

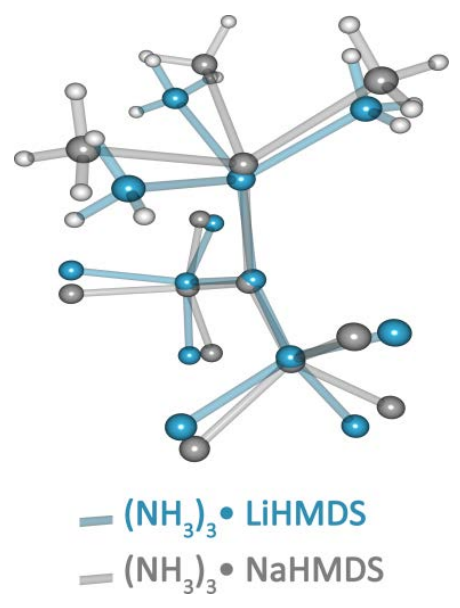

d)

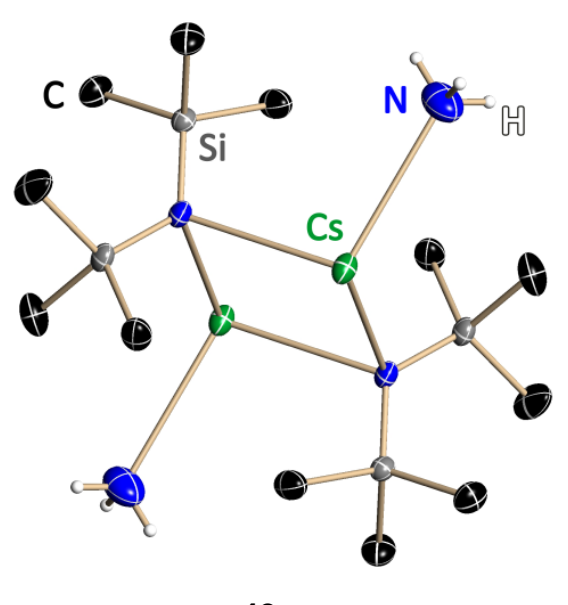

49

Fig. 1- 6. a) Hydrogen bonded monomers of two neighboring $\left(\mathrm{NH}_{3}\right)_{3} \cdot \mathrm{M}^{1} \mathrm{~N}\left(\mathrm{SiMe}_{3}\right)_{2}\left[\mathrm{M}^{1}=\mathrm{Li}(45), \mathrm{Na}(46)\right]$ molecules. b) Both compounds crystallize with the same aggregation motif, but in different space groups. 45 crystallizes in the monoclinic space group $C 2 / c$ and 46 in the primitive monoclinic space group $P 2_{1} / c$. The superposition plot of the asymmetric units shows that both structures do not fit perfectly. Apart from the different $\mathrm{M}-\mathrm{N}_{\text {amide }}$ bond lengths both trimethylsilyl groups and the $\mathrm{NH}_{3}$ molecules are twisted slightly with respect to another. c) Crystal structure of tetrasolvated $\left[\left(\mathrm{H}_{3} \mathrm{~N}\right)_{2} \mathrm{M}^{2} \mathrm{~N}\left(\mathrm{SiMe}_{3}\right)_{2}\right]_{2}\left[\mathrm{M}^{2}=\mathrm{K}(47), \mathrm{Rb}(\mathbf{4 8})\right]$. d) Crystal structure of disolvated CsHMDS dimer 49. Anisotropic displacement parameters are depicted at the $50 \%$ probability level. Carbon bound hydrogen atoms are omitted for clarity. ${ }^{[1]}$

\footnotetext{
${ }^{4}$ Includes revised parts of my publication: R. Neufeld, R. Michel, R. Herbst-Irmer, R. Schöne, D. Stalke,
} Chem. Eur. J. 2016, submitted. 
Like others ${ }^{[54]}$ we decided for ammonia as a convenient solvent as we realized earlier that the structure determining effect by single ammonia molecules is much less pronounced than by e.g. bi- or tridentate donor bases and the probability to isolate monomeric contact ion pairs (CIPs) or even solvent separated pairs (SSIPs) is much higher. ${ }^{[7,55]}$ Page et al. showed for example that ammonia is a promising candidate to replace dipolar aprotic solvents like e.g. DMSO and DMF in a number of industrial processes. ${ }^{[56]} \mathrm{NH}_{3}$ has a boiling point of $-33{ }^{\circ} \mathrm{C}$ and a vapor pressure of 9 bar at $24{ }^{\circ} \mathrm{C},{ }^{[57]}$ so it is much easier to remove or rather to recover, compared to many toxic and harmful chelating solvents like e.g. PMDETA, unwanted in any pharmaceutical or natural product process. The physical properties of liquid ammonia are similar to those of liquid water. $\mathrm{NH}_{3}$ is capable to solve many synthetically useful saltr like e.g. $\mathrm{LiNO}_{3}, 244 \mathrm{~g} / 100 \mathrm{~g}$ at $25^{\circ} \mathrm{C}$ because of its small dielectric constant $\left(\mathrm{NH}_{3}: 16.9, \mathrm{H}_{2} \mathrm{O}: 78.3\right.$ at $\left.25^{\circ} \mathrm{C}\right) .{ }^{[58]}$ With a $p \mathrm{~K}_{\mathrm{a}}$ value of 27.7 at $25^{\circ} \mathrm{C}^{[59]}$ it is not exposed to fast auto protolysis like water $\left(p \mathrm{~K}_{\mathrm{a}}=15.7\right.$ at $\left.25^{\circ} \mathrm{C}\right) .{ }^{[60]} \mathrm{A}$ high dipole moment $(\mu=1.47)$, ${ }^{[61]}$ a small steric demand (compared to e.g. THF, $\mathrm{Et}_{2} \mathrm{O}$, etc.) and a high electron density at the nitrogen atom makes ammonia an advantageous donor base for many metal ions especially for alkali metal ions. ${ }^{[7,55 b, 62]}$ Instead of using net liquid ammonia is also feasible to use it as an additive to classic solvents like THF or toluene by introducing gaseous $\mathrm{NH}_{3}$ to the solution. ${ }^{[7,55 b]}$ Monomeric LiHMDS with monodentate donor bases was only characterized in solution. Anyway, since the first preparation of LiHMDS in 1959 by Wannagat and Niederprüm, ${ }^{[63]}$ all efforts to crystalize monomeric LiHMDS in the absence of chelating ligands failed. In 2016, Neufeld et al. succeeded for the first time the crystallization of the missing monomeric key compound. ${ }^{[1]}$ With ammonia as donor base trisolvated LiHMDS 45 and NaHMDS 46, showing unique hydrogen bond interactions between two metal HMDS monomers, have been characterized. In addition, unprecedented tetrasolvated K- 47 and RbHMDS-dimers 48 as well as disolvated CsHMDS-dimer 49 with very close intermolecular $\mathrm{Si}-\mathrm{CH}_{3}$ …Cs s-block "agostic" interactions have been prepared and characterized by single-crystal X-ray structure analysis. ${ }^{[1]}$ 


\subsubsection{Structure of Hauser- and Turbo-Hauser Bases ${ }^{5}$}

Although there is a great deal of information on the utility of these reagents, very little is known regarding the nature of (Turbo-)Hauser bases in solution. One reason for that lack of information is that Hauser bases show a complex behaviour in solution. It was proposed $^{[64]}$ that it could be similar to the Schlenk-equilibrium of Grignard reagents in ether solution, where more than one magnesium containing species exists.

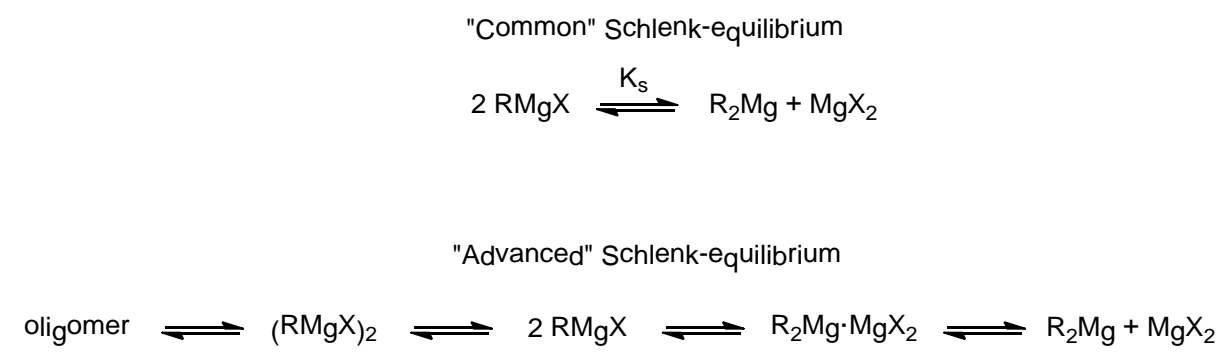

Scheme 1-8. The Schlenk-equilibria.

A rearrangement of the organic ligand takes place and ends up in an equilibrium with the diorganomagnesium and the magnesiumdihalide (Eqn. I in Scheme 1-8). ${ }^{[65]}$ Later, molecular association studies also revealed oligomeric Grignard structures in diethyl ether. This fact complicated the simple Schlenk-equilibrium (I) so oligomeric species would have to be included (Eqn. II in Scheme 1-8). ${ }^{[66]}$ There are a few known solid state structures of Grignard complexes. ${ }^{[67]}$ It was found that the $\mathrm{Mg}$ atoms are predominantly tetrahedrally coordinated and dimeric species are bridged through halide atoms. Especially in the late 60 s a lot of solution structure investigations have been done mainly by ebullioscopic- ${ }^{[66,68]}$ calorimetric $^{[69]}$ and $\mathrm{NMR}^{[70]}$ measurements. The position of the Schlenk-equilibrium is considered to be dependent on the nature of the solvent, the steric of the organic substituent, the type of halogen involved and the temperature. ${ }^{[71]}$ In THF, all alkyl and arylmagnesium halides $(\mathrm{Cl}, \mathrm{Br}, \mathrm{I})$ are found to be monomeric over a wide concentration range. ${ }^{[66,69]}$ In diethyl ether, alkyl and aryl Grignard reagents are mostly monomeric at low concentrations (less than $0.5 \mathrm{M}$ ) and mostly dimeric at higher concentrations (0.5$1.0 \mathrm{M}){ }^{[68 \mathrm{a}]}$

\footnotetext{
${ }^{5}$ Includes revised parts of my publication: R. Neufeld, T. L. Teuteberg, R. Herbst-Irmer, R. A. Mata, D. Stalke, JACS 2016, submitted.
} 


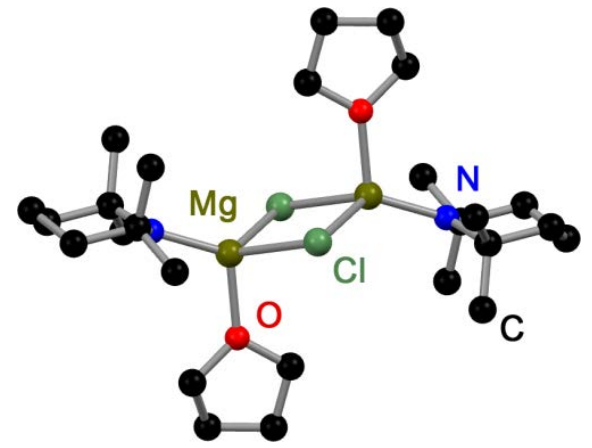

50

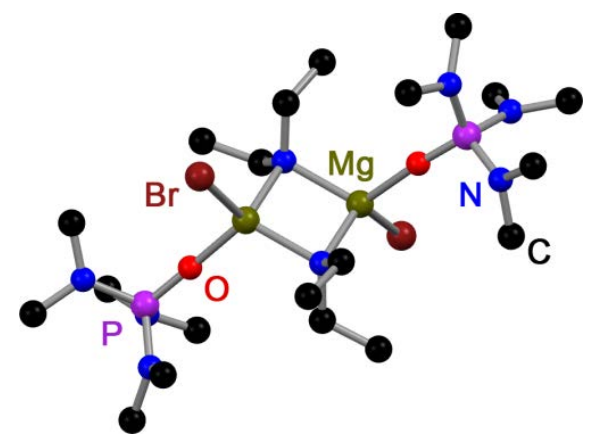

52

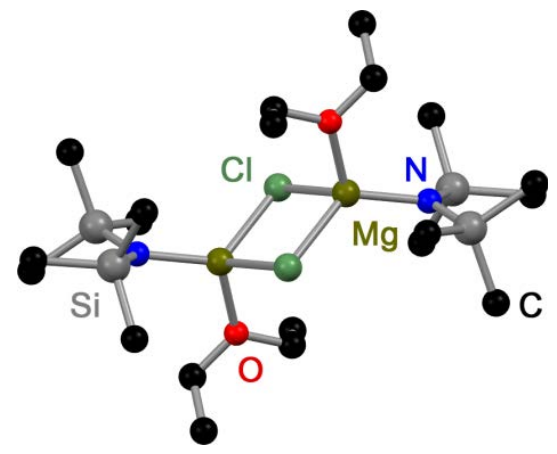

51

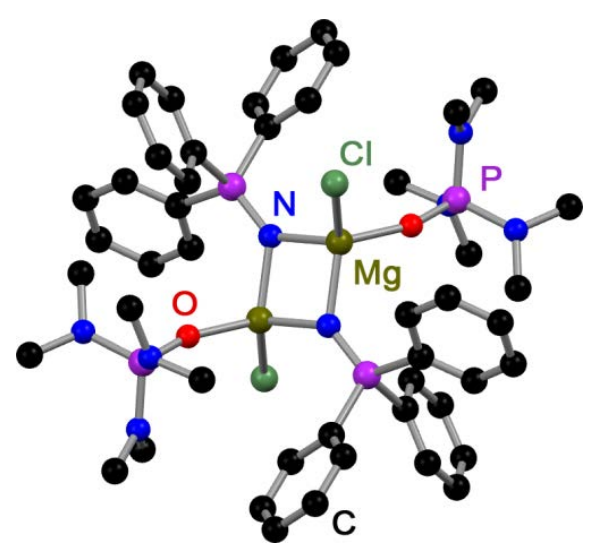

53

Fig. 1-7. Selected structures of dimeric Hauser bases in the solid state with hydrogen atoms omitted for clarity.

However, surprisingly there are no investigations concerning the aggregation of Hauser bases in solution. In the solid state $\operatorname{TMP}^{[72]}(\mathbf{5 0})$ and $\operatorname{HMDS}^{[73]}(\mathbf{5 1})$ Hauser bases as well as all Grignard dimers ${ }^{[67 a, 67 b, 67 e, 74]}$ are bridged by halides (Fig. 1-7). In contrast to Grignard reagents, dimeric amido bridged (Turbo)Hauser bases exist, too. ${ }^{6}$ All have one feature in common: They are bridged by less bulky amido ligands like $\mathrm{Et}_{2} \mathrm{~N}^{-[73 a]}(52), \mathrm{Ph}_{3} \mathrm{P}=\mathrm{N}^{-[75]}$ (53) and ${ }^{i} \operatorname{Pr}_{2} \mathrm{~N}^{-[28]}$ (9) (Fig. 1-7 and Fig. 1-8). At least in the solid state, it can be concluded that the displacement to halide bridges may be influenced by bulky groups on the amide ligand. ${ }^{[64]}$ However, solid state structures may not necessarily maintain in solution. The impact of $\mathrm{LiCl}$ on the solution structure of Grignard reagents and Hauser bases is still unclear. Noteworthy is that the diisopropylamido reagent ${ }^{i} \mathrm{Pr}_{2} \mathrm{NMgCl} \cdot \mathrm{LiCl} 9$ shows a much lower reactivity than its TMP counterpart 10. That difference in reactivity was shown by the deprotonation of isoquinoline in THF solution (Scheme 1-9). While TMPMgCl-LiCl 10 required only $2 \mathrm{~h}$ and 1.1 equivalents, 9 needed $12 \mathrm{~h}$ and 2 equivalents for comparable metalation (54). ${ }^{[23]}$

\footnotetext{
${ }^{6}$ Cambridge Structural Database CSD, version 5.36 (Updated Nov 2014), chelating amido ligands have been
} excluded. 


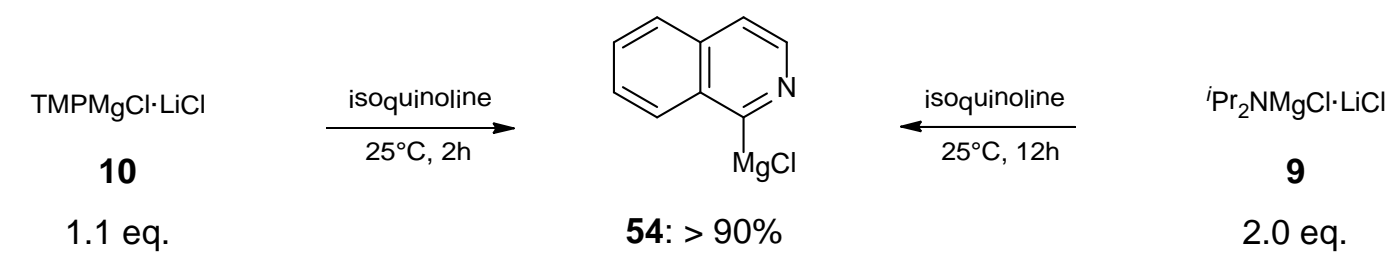

Scheme 1-9. Contrasting reactivity of Turbo-Hauser base 9 and $\mathbf{1 0}$ for ortho metalation of isoquinoline. ${ }^{[23]}$

The differing reactivity could be a result of unequal aggregation states reflected in a different solubility of both reagents. The solubility of TMPMgCl$\cdot \mathrm{LiCl} 10$ is $1.2 \mathrm{M}$, while that of ${ }^{i} \mathrm{Pr}_{2} \mathrm{NMgCl} \cdot \mathrm{LiCl} 9$ is only half of it $(0.6 \mathrm{M}) \cdot{ }^{[23]}$ Actually, in the solid state compound 10 exists as a monomeric dinuclear contact ion pair (CIP) ${ }^{[72]}$ and compound 9 as a dimeric tetranuclear $\mathrm{CIP}^{[28]}$ bridged by two amide ligands (Fig. 1-8). In both structures $\mathrm{LiCl}$ coordinates to the magnesium amides.
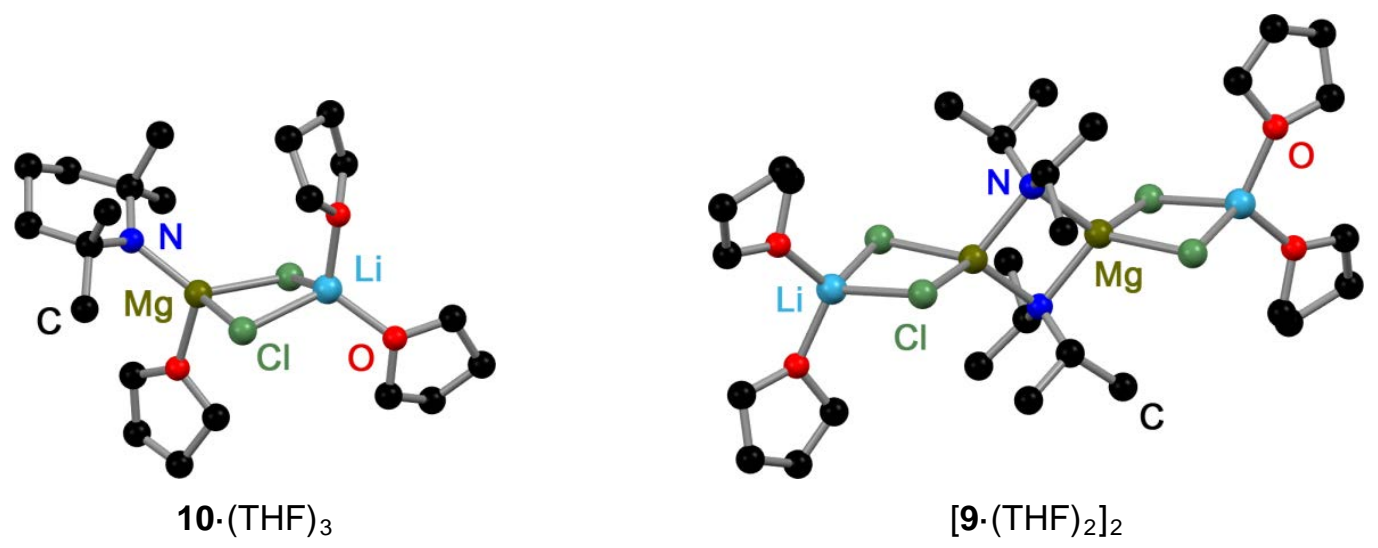

Fig. 1-8. Solid state structures of 10.(THF $)_{3}$ and $\left[\mathbf{9 \cdot}(\mathrm{THF})_{2}\right]_{2}$ with hydrogen atoms omitted for clarity.

However, it is not clear whether the structures aggregated by $\mathrm{LiCl}$ are really stable in solution or just transient species. Knochel has speculated that $\mathrm{LiCl}$ deaggregates $\mathrm{RMgX}$ oligomers $^{[23]}$ and forms a more reactive bimetallic monomer $\mathrm{RMgCl} \cdot \mathrm{LiCl}$ that is supposed to give magnesiate character to the Grignard reagent in the sense of a solvent separated ion pair (SSIP) $\left[\mathrm{Li}(\mathrm{THF})_{4}\right]^{+}\left[\mathrm{RMg}(\mathrm{THF}) \mathrm{Cl}_{2}\right]^{-} \cdot{ }^{[76]}$ Garcia-Álvarez and Mulvey et al. analyzed ${ }^{[28]}$ crystals of 9 and 10 in THF- $d_{8}$ solution at $-50^{\circ} \mathrm{C}$ by employing the diffusion coefficient formula weight (D-FW) analysis that was pioneered by Williard et al. (see chapter 1.2.7.2). ${ }^{[77]}$ Because of signal overlapping problems, the authors had to use inappropriate references, so the molecular weight (MW) determination was prone to a relatively high error of approximately $\pm 30 \%$. This is why they were not able to "clearly establish the exact nature of the solution species". ${ }^{[28]}$ However, the first key conclusion was that the molecular structure of both Turbo-Hauser bases were not retained in THF- $d_{8}$ solution and the second 
was that a SSIP situation like it was proposed by Knochel et al., described by negative charged magnesium ate complexes (like e.g. $\left[\mathrm{RMg}(\mathrm{THF}) \mathrm{Cl}_{2}\right]^{-}$) and free $\left[\mathrm{Li}(\mathrm{THF})_{4}\right]^{+}$ seemed most probable. ${ }^{[28]}$

\subsection{Diffusion NMR Measurements}

Like already mentioned in the previous chapters, chemists have always had a vital interest in the size and aggregation state of molecules in solution. NMR measurements of selfdiffusion coefficients have gained an increasing importance in this area. The physical observable that can be derived from these measurements is the diffusion coefficient $D$ that is sensitive to size, shape and density of the molecular species. ${ }^{[6]}$ In the mid-1960s Stejskal and Tanner developed the pulsed field gradient spin-echo (PFG-SE) sequence that enables the measurement of diffusion coefficients in solution. ${ }^{[78]}$ However, it took at least 30 years until diffusion measurements have become routinely accessible through the introduction of conventional high-resolution NMR spectrometers that are equipped with pulsed field gradient probe heads. Field gradients are necessary to encode the physical location of a molecule and therefore to measure its diffusion in solution. In the beginning of the 1990s a huge varieties of NMR diffusion methods and applications have been developed and applied which were reviewed in several articles. ${ }^{[79]}$ These measurements include applications rising from the fields of biology and pharmacy, ${ }^{[80]}$ polymeric, ${ }^{[81]}$ organic $^{[82]}$ and inorganic $^{[83]}$ chemistry. In the following sections a theoretical background will be given starting from the aspects of diffusion, followed by measuring self-diffusion by applying different pulse sequences and finally the advanced process in molecular weight determination from diffusion NMR measurements will be discussed.

\subsubsection{Aspects of Diffusion}

Self-diffusion arises from random translational motion of molecules in homogeneous solutions (Brownian motion) driven by the thermal energy of the system. This motion may be characterized by the so called self-diffusion-coefficient $D$. The average displacement of a molecule in three dimensions is zero over time, but the mean square displacement is nonzero. The distance of a molecule travelled in a single direction is given by:

$$
Z_{\mathrm{rms}}=\sqrt{2 D t}
$$


where $z_{\text {rms }}$ is the root mean square distance of an ensamble of molecules travelled in time $t$. This is why diffusion coefficients have units of $\mathrm{m}^{2} / \mathrm{s}$.

The probability $(P)$ of finding a particle at position $r$, from the starting position $r_{0}$, over a time $t$, results in a Gaussian function which is a solution to Fick's second law:

$$
P\left(r_{0}, r, t\right)=A \exp \left(\frac{-\left(r-r_{0}\right)^{2}}{4 D t}\right)
$$

where A represents a geometric volume normalization $A=(4 \pi D t)^{3 / 2} \cdot{ }^{[79 b]}$ The PFG-SE diffusion experiment works by expressing $P$ in Eqn. (2) as a function of the nuclear spin phase $\varphi$, instead of the position $r$. The de-phasing caused by pulsed field gradients and the movement of the molecules during the time $t$, results after re-phasing in a NMR signal intensity attenuation that can be directly related to the diffusion coefficient $D .{ }^{[79 b]}$

As already mentioned, diffusion depends on size and shape of molecules which can be shown by the Stokes-Einstein equation:

$$
D=\frac{k_{\mathrm{b}} T}{6 \pi \eta r_{\mathrm{s}}}
$$

where $k_{\mathrm{b}}$ is the Boltzmann constant, $T$ the absolute temperature, $\eta$ the solvent viscosity and $r_{\mathrm{s}}$ the Stokes radius of a spherical particle that has to be much larger than the solvent molecule in a fluid continuum. ${ }^{[84]}$ Often $r_{\mathrm{s}}$ is equalized with the hydrodynamic radius $r_{\mathrm{H}}$ of an equivalent sphere that diffuses with the same diffusion coefficient as the diffusing particle. However, this approximation has to be considered carefully. The scaling factor 6 in Eqn. (3) is invalid for smaller molecules, where the hydrodynamic radius of the solute tends towards that of the solvent molecule and therefore has to be corrected (a detailed description is presented in section 1.2.7.1).

\subsubsection{The Hahn Spin-Echo Experiment}

The NMR signal that is observed after an initial $90^{\circ}$ pulse (Fig. 1-9 and Fig. 1-10b) decays with time due to variations in the magnetic field. Inhomogeneities in the external field due to intramolecular interactions lead the spins to process at different rates that result in a loss of magnetization (dephasing due to $T_{2}$ spin-spin relaxation, Fig. 1-10c). However, this 
dephasing effect can be removed by applying an additional $180^{\circ}$ pulse around the $y$ axis (after a time $\tau$ ) that inverts the relative spin positions (Fig. 1-10d). Because each spin continues to process with its former frequency, all spins rephase perfectly at time $2 \tau$ forming a spin echo (SE, Fig. 1-10e). ${ }^{[85]}$

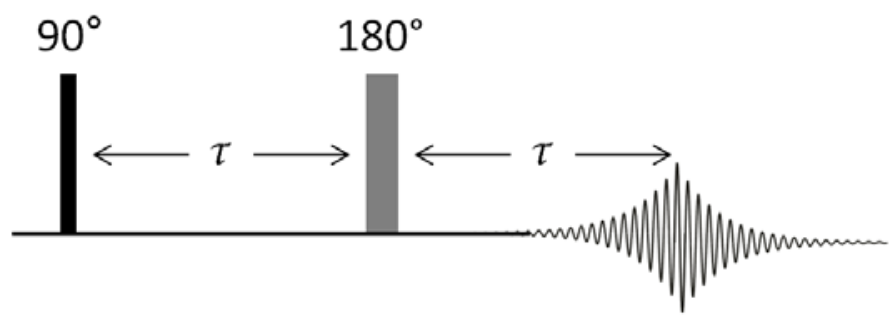

Fig. 1-9. Schematic presentation of the Hahn spin-echo pulse sequence.
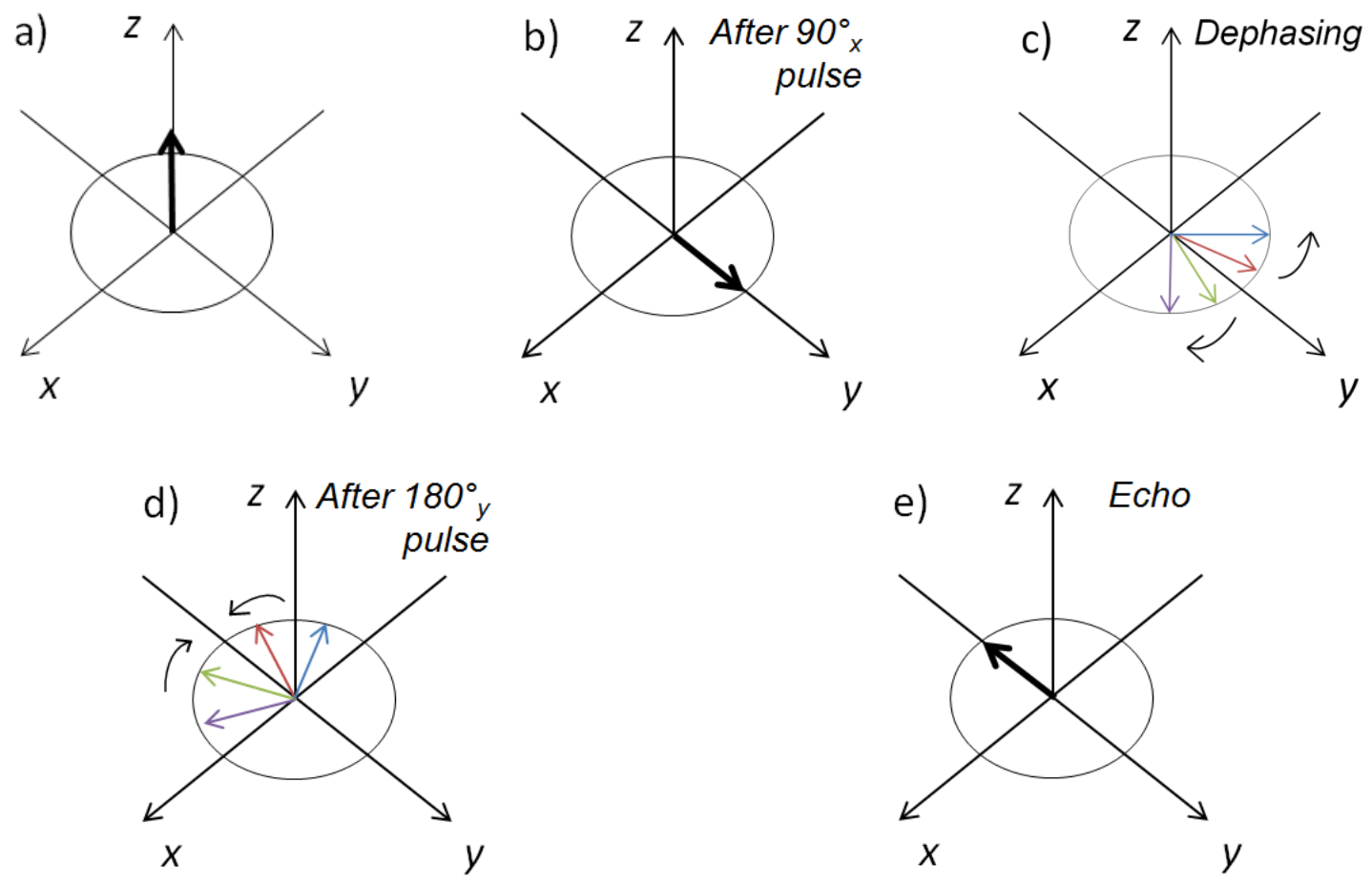

Fig. 1-10. Schematic representation of the Hahn spin echo (SE) pulse sequence and its effects on the spin system.

\subsubsection{The Pulsed Gradient Spin-Echo (PFG-SE)-Experiment}

Before starting with the diffusion experiments, first the effect of gradients on the nuclear spin frequency is described. A gradient is an external magnetic field $B$ whose strength 
changes with position. Today, most commercially available NMR spectrometers include self-shielded gradient coils for use e.g. in automatic shimming experiments. The additional magnetic field $B_{\mathrm{z}}$ is applied along the $\mathrm{z}$-axis that can be described by:

$$
G_{\mathrm{z}}=\frac{\partial B_{\mathrm{z}}}{\partial z}
$$

Assuming that the $B_{\mathrm{z}}$ gradient is constant, the effective Larmor frequency $\omega_{\text {eff }}$ is:

$$
\omega_{\text {eff }}(z)=\gamma\left(B_{0}+G_{\mathrm{z}} \cdot z\right)
$$

where $\gamma$ is the gyromagnetic ratio, $B_{0}$ the strength of the homogeneous, external static magnetic field and $G_{z}$ the gradient strength of the additional inhomogeneous magnetic field. Eqn. (5) shows that the effective Larmor frequency increases when external gradient fields are applied. Due to the inhomogeneity of the latter, the spin frequency varies linearly along the $\mathrm{z}$-axis over the whole sample (Fig. 1-11). ${ }^{[79 b]}$

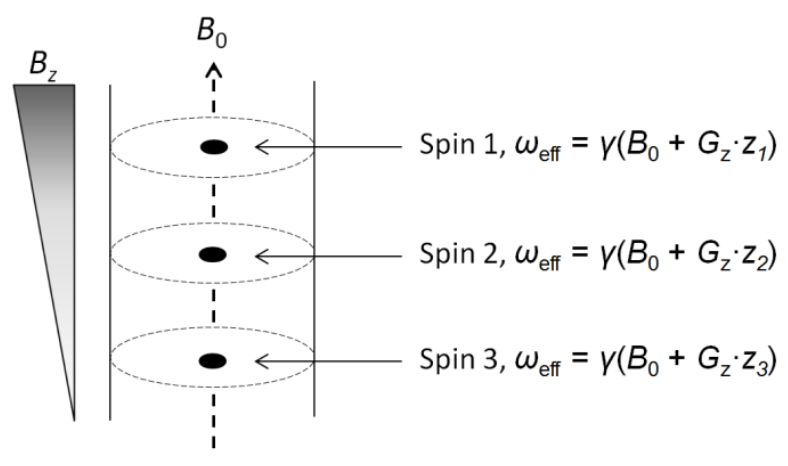

Fig. 1-11. The influence of the external gradient field on the Larmor frequency distribution. The total magnetic field is the sum of the homogeneous static magnetic field and the applied gradient field.

The Hahn spin-echo (SE) pulse sequence, in combination with pulse field gradients (PFG), results in a pulsed field gradient spin-echo (PFG-SE)-experiment that is the cornerstone of NMR diffusion experiments. In Fig. 1-12 a schematic picture of the PFG-SE sequence is displayed. 


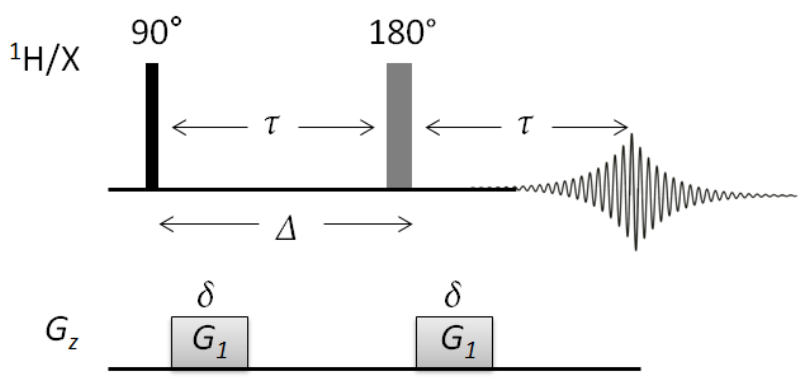

Fig. 1-12. The basic sequence for monitoring self-diffusion (PFG-SE sequence). The diffusion during the period $\Delta$ is obtained by measuring a series of experiments with increasing gradient strengths.

Without gradient pulses this sequence displays a standard spin-echo experiment (Fig. 1-9). When the first gradient $G_{z}$ (encoding gradient) with the length $\delta$ is applied, the magnetization vector will impose a spatially dependent phase, which can be refocused by a second gradient of equal duration and magnitude. Due to the $180^{\circ}$ pulse between them, the effect of the gradient pulses would cancel. However this is only true, if the spins of a molecule remain in the same physical position. If the molecule diffuses away from its initial position during the diffusion delay $\Delta$, then the local field experienced during the second gradient (decoding gradient) does not exactly match with the first PFG. This scenario leads to an attenuation of the spin echo (SE) signal.

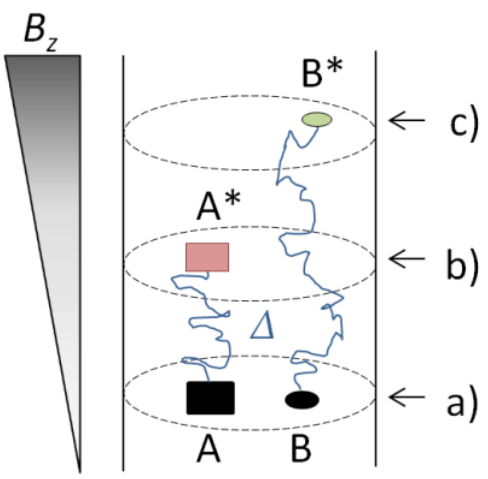

Fig. 1-13. Schematic representation of the spin echo signal attenuation through PFG and molecular diffusion. a) If no diffusion occurrs than the spins of molecule $A$ and B would refocus after the PFG-SE pulse sequence resulting in a full intensity of the spin echo. b) Due to diffusion, the local field experienced by molecule A does not match that experienced during the secound gradient puls $\left(A^{\star}\right)$. The spins do not fully refocus. This provides a reduced magnetization in respect to the z-axis and therefore in a redused SE intensity. c) Greater attanuation is observed for the faster diffusing molecule B that signal attenuation is even bigger than that of molecule A. 


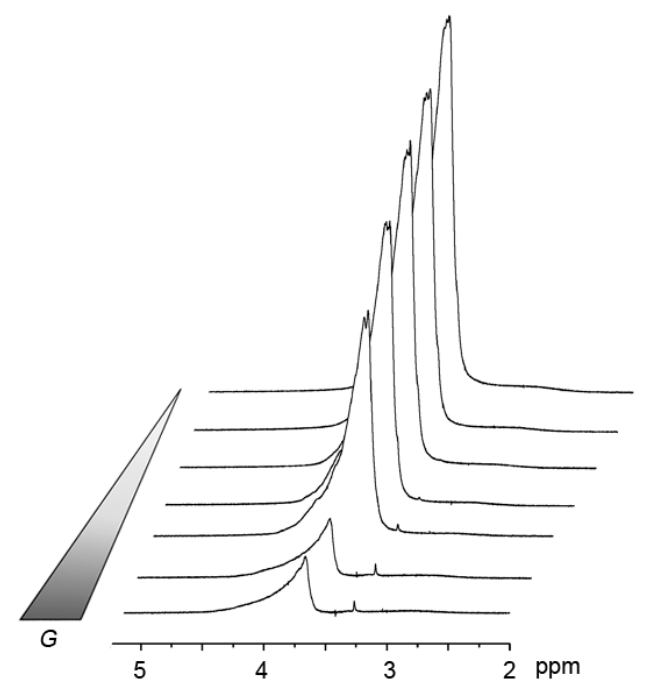

Fig. 1-14. Experimental $1 \mathrm{D}{ }^{1} \mathrm{H}$ diffusion traces. The intensity decay is a result of a progressively increased gradient strength $G$.

To characterize diffusion rates, it is necessary to progressively increase the parameter $\Delta, \delta$ or the strength of the gradient pulses $G_{z}$ to monitor the change in the SE intensities. However, since longer diffusion times provide additional signal loss due to relaxation processes, it is more advantageous to vary the gradient strengths $G$ instead of $\Delta$ and $\delta$ (Fig. 1-14).

At the end of a PFG-SE experiment, the observed signal intensity $I_{\mathrm{G}}$ is given by the socalled Stejskal-Tanner equation: ${ }^{[78]}$

$$
I_{\mathrm{G}}=I_{0} \exp \left(-\frac{2 \tau}{T_{2}}\right) \exp \left[-(\gamma \delta G)^{2} D\left(\Delta-\frac{\delta}{3}\right)\right]
$$

where $I_{0}$ is the signal intensity without the gradient spin-echo, $\tau, \delta$ and $\Delta$ the delays as displayed in Fig. 1-12, $T_{2}$ is the transverse relaxation rate constant, $\gamma$ is the gyromagnetic ratio, $G$ is the gradient strength and $D$ is the diffusion coefficient. For a typical PFG-SE experiment, where the gradient strength $G$ is varied and the total echo time $2 \tau$ is constant a more useful equation is: 


$$
I_{\mathrm{G}}=I_{\mathrm{G}=0} \exp \left[-(\gamma \delta G)^{2} D\left(\Delta-\frac{\delta}{3}\right)\right]
$$

Since the constants $\gamma, \delta$ and $\Delta$ are known, the diffusion coefficient $D$ can be calculated directly from linear or non-linear regression. Optionally the data can be presented in the pseudo-2D-DOSY format (Fig. 1-17 in chapter 1.2.6).

\subsubsection{The Pulsed Gradient Stimulated-Echo (PFG-STE)-Experiment}

In the above described PFG-SE-experiment the magnetization is transverse during diffusion. Therefore this sequence depends highly on transverse (spin-spin) relaxation times $T_{2}$ (see Eqn. (6)).

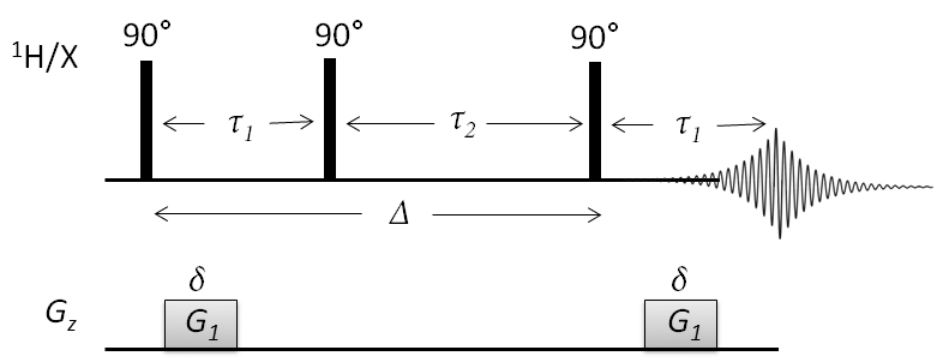

Fig. 1-15. PFG stimulated-echo (PFG-STE)-sequence

This relaxation loss can be very problematic, because very large molecules require long diffusion delays $(\Delta)$ and simultaneously show increased $T_{2}$ relaxation rates. In the alternative PFG stimulated-echo (PFG-STE)-sequence a second $90^{\circ}$ pulse sets the magnetization along the $\mathrm{z}$-axis during the diffusion encoding time (Fig. 1-15). ${ }^{[86]}$ In this period $\left(\tau_{2}\right)$ the relaxation is dictated by the potentially slower longitudinal (spin-lattice) relaxation rate $T_{1}$, while $T_{2}$ relaxation is only present during the relatively short gradient periods $\tau_{1}$. After the diffusion period a third $90^{\circ}$ pulse returns the magnetization with reversed sign to the transverse $x-y-p l a n e$ (Fig. 1-10), so the spin moments can refocus again. The observed signal intensity for the stimulated echo (STE) is given by: 


$$
I_{\mathrm{G}}=\frac{I_{0}}{2} \exp \left(-\frac{2 \tau_{1}}{T_{2}}-\frac{\tau_{2}}{T_{1}}\right) \exp \left[-(\gamma \delta G)^{2} D\left(\Delta-\frac{\delta}{3}\right)\right]
$$

When the gradient strength is the only varying parameter, again Eqn. (7) can be used to describe the STE-intensity. Compared with the PFG-SE-sequence (Eqn. (6)) it may be seen that the signal intensity is reduced by a factor 2 (Eqn. (8)), because shortly after the second $90^{\circ}$ pulse a portion of the magnetization dissipates due to transverse relaxation. ${ }^{[87]}$ This is why the difference between $T_{1}>T_{2}$ is important and the initial signal has to be strong enough to allow a sufficient PFG-STE-experiment.

\subsubsection{Advancements to the Stimulated-Echo}

Over the years, the PFG-STE sequence led to the development of advanced diffusion NMR pulse sequences with the aim to minimize lineshape distortions, to reduce experiment time and to suppress convection disturbance. In following, the benefits of some advanced pulse sequences will be displayed in a sense of a short overview:

1) BPP-LED: The key feature of this sequence is the replacement of single gradient pulses in the STE sequence by a pair of accurately matched pulses having different polarities that are separated by a $180^{\circ}$ pulse. These so called bipolar pulse pairs (BPP) reduce eddy current distortions and minimize residual background gradients that lead to reduced lineshape disturbances. ${ }^{[88]}$ In addition the "longitudinal-eddycurrent delay" (LED) pulse sequence reduces also effects of eddy currents in diffusion measurements by introducing an eddy current delay period prior to detection. ${ }^{[89]}$

2) ONE-SHOT: The use of balanced asymmetrical bipolar gradient pairs in addition to purge gradients leads to rapid diffusion measurements and often to higher signal-to-noise ratios (compared to the BPP-LED pulse sequence). ${ }^{[90]}$

3) D-STE: The so called "double-stimulated-echo" experiment is the most effective sequence that cancels the unwanted effect of convection during the diffusion measurement by dividing the total diffusion period in two STE sequences. ${ }^{[91]}$ However, the signal intensity is reduced by an additional factor of 2 . 
4) Pure Shift: The Zangger-Sterk pulse sequence suppresses multiplet structures in the spectral dimension that minimize signal overlap problems. ${ }^{[92]}$

\subsubsection{Extracting Diffusion Coefficients}

Once the diffusion data set is collected (typically consisting as a set of 16-32 1D spectra) the diffusion coefficients of the species of interest have to be extracted from the data. The data analysis relies on fitting the attenuated intensity of a component as a function of the applied gradient strength. The diffusion coefficient $D$ can be extracted either from nonlinear Gaussian- (Fig. 1-16a), exponential- (Fig. 1-16b) or linear- (Fig. 1-16c) regression fits. However, because of a less accuracy of $\ln \left(I / I_{0}\right)$ the linear fits are less accurate than the non-linear fits.

a)

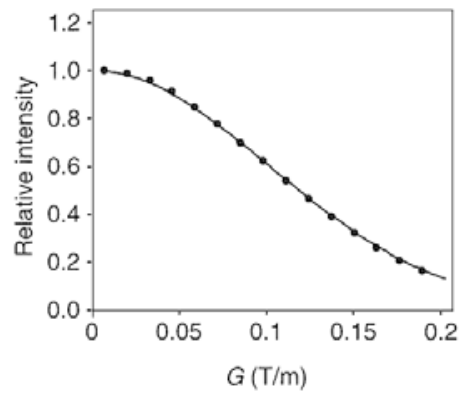

b)

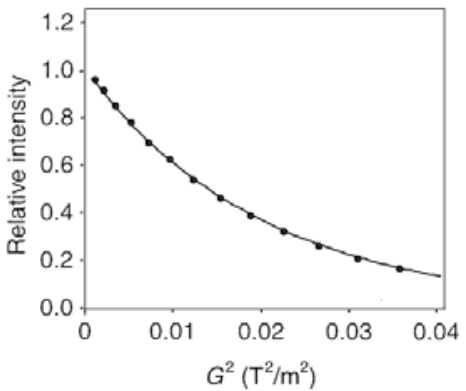

c)

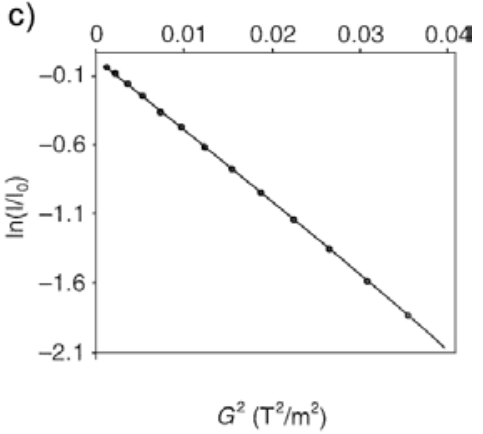

Fig. 1-16. Regression fit analysis to extract the diffusion coefficient by fitting a) $I_{\mathrm{G}}$ vs. $G$ (Gaussian decay profile); b) $I_{\mathrm{G}}$ vs. $G^{2}$ (exponential decay profile) and c) $I_{\mathrm{G}} / I_{\mathrm{G}=0}$ vs. $G^{2}$ (linear decay profile).

A highly aesthetic presentation of the diffusion data is the pseudo 2D spectrum introduced by Morris and Johnson in 1992. ${ }^{[93]}$ The first dimension represents the regular chemical shifts $\delta$ and the second dimension gives the diffusion coefficients $D$ of species separated by their particle size (Fig. 1-17). The authors referred this pseudo 2D experiment as "Diffusion-Ordered NMR Spectroscopy" (DOSY). ${ }^{[93]}$ Typically the diffusion coefficients are extracted from exponential fits that are described above, while the peak width reflects the magnitude of the fitting error. Since its invention in 1992, the DOSY "NMR Chromatography" experiment has gained huge popularity as an advanced tool for identifying individual species in a multicomponent solution (Fig. 1-18). 


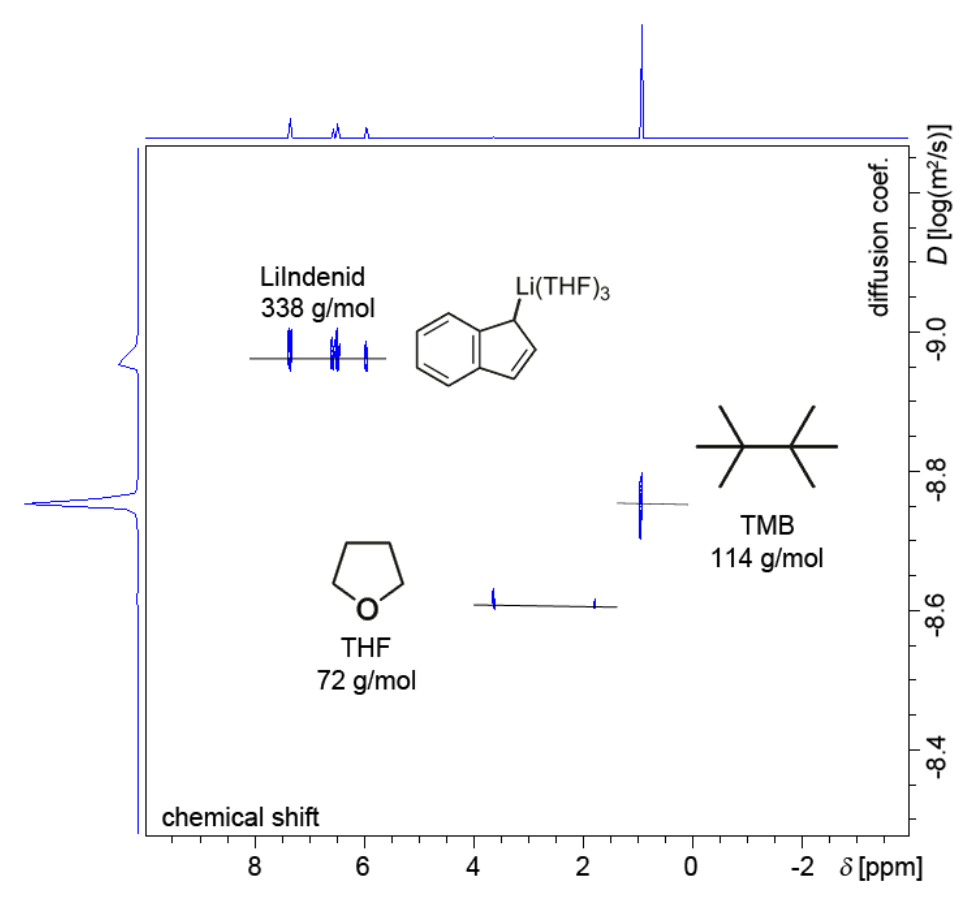

Fig. 1-17. Example of a pseudo 2D-DOSY-spectrum with three compounds with unequal sizes and therefore different diffusion coefficients.

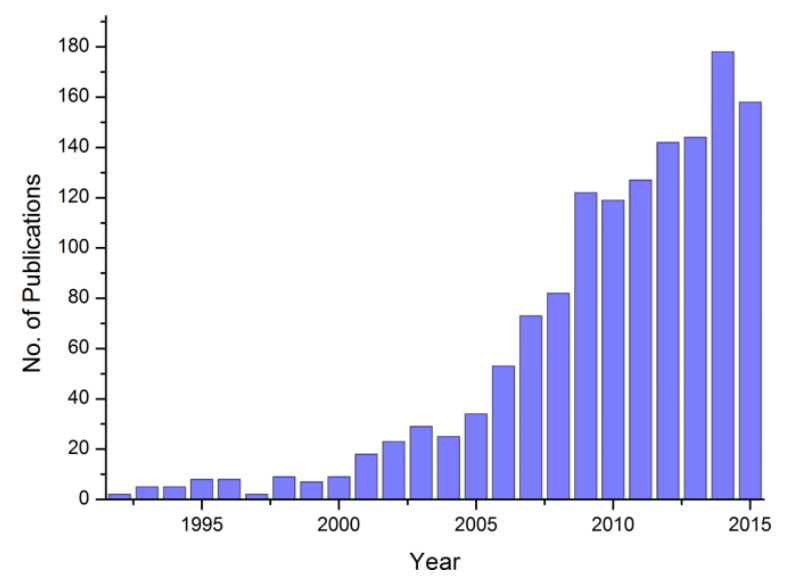

Fig. 1-18. Number of publications using the concept of DOSY. ${ }^{7}$

It is essentially important that signals show a desirable distribution, so no signal overlap is present. However, for weak overlap a range of more complicated mathematical procedures like e.g. CONTIN, DECRA and CORE are available. In cases of high signal overlap the apparent diffusion coefficient will be an intermediate result of both components. An alternative for signal overlapping problems may be the use of single shift pulse sequences ${ }^{[92]}$ or hybrid diffusion sequences such as COSY-IDOSY ${ }^{[94]}$, HSQC-IDOSY[95] or TOCSY$\operatorname{DOSY}^{[96]}$ (so called 3D-DOSY experiments; IDOSY means the implementation of an

${ }^{7}$ Derived from a scifinder ${ }^{\circledast}$ search with the keyword "DOSY". 
INEPT transfer into a BPP-STE sequence, where the signal detection is performed on the heteroatom while the diffusion encoding is performed on ${ }^{1} \mathrm{H}$ magnetization).

\subsubsection{Molecular Weight Determination}

\subsubsection{Molecular Weights Derived from the Stokes-Einstein Equation}

Usually species are identified by comparing the hydrodynamic radius of a reference molecule with that of the solute. The hydrodynamic radius $r_{s}$ of the solute can be directly calculated from the Stokes-Einstein equation:

$$
r_{\mathrm{s}}=\frac{k_{\mathrm{b}} T}{6 \pi \eta D_{\mathrm{s}}}
$$

where $k_{\mathrm{b}}$ is the Boltzmann constant, $T$ the absolute temperature, $\eta$ the solvent viscosity and $D_{s}$ is the diffusion coefficient. ${ }^{[84]}$ For a quantitative relation of the hydrodynamic radius with the molecular weight (MW) of a particle the following equation can be used: ${ }^{[97]}$

$$
r_{\mathrm{s}}=\left(\frac{3 M v}{4 \pi N_{\mathrm{A}}}\right)^{\frac{1}{3}}
$$

where $N_{\mathrm{A}}$ is the Avogadro constant, $M$ is the MW and $v$ is the partial specific volume of the particle. Further it is possible to relate the diffusion coefficients of two molecules $D_{\mathrm{s}}$ and $D_{\text {ref }}$ to their MWs $M_{\mathrm{s}}$ and $M_{\text {ref: }}{ }^{[97]}$

$$
\frac{D_{\mathrm{s}}}{D_{\mathrm{ref}}}=\left(\frac{M_{\mathrm{ref}}}{M_{\mathrm{s}}}\right)^{\frac{1}{3}}
$$

However, equations (9) to (11) are only true for ideal spherical particles that have to be much larger than the solvent in a fluid continuum. For non-spherical molecules with a slightly larger size than the solvent, the additional factors $c_{s}$ for size- and $f_{s}$ for shape correction, respectively have been added to the Stokes-Einstein equation: ${ }^{[79 c]}$

$$
r_{\mathrm{s}}=\frac{k_{\mathrm{b}} T}{c_{\mathrm{s}} f_{\mathrm{s}} \pi \eta D_{\mathrm{s}}}
$$


Gierer and Wirtz (Eqn. (13)) $)^{[98]}$ and Chen et al. (Eqn. (14)) $)^{[99]}$ derived the factor $c_{\mathrm{s}}$ as a function of $r_{\text {solv }} / r_{\mathrm{s}}$ by microfrictional theory and semi-empirically methods (Fig. 1-19a):

$$
\begin{gathered}
c_{\mathrm{S}}(\mathrm{GW})=\frac{6}{\frac{3 r_{\mathrm{solv}}}{2 r_{\mathrm{s}}}+\frac{1}{1+\frac{r_{\mathrm{solv}}}{r_{\mathrm{s}}}}} \\
c_{S}(\text { Chen })=\frac{6}{1+0.695\left(\frac{r_{\text {solv }}}{r_{s}}\right)^{2.234}}
\end{gathered}
$$

The dependence of $c$ on $r_{s}$ taking methylene chloride as solvent is demonstrated in Fig. 119a. Further the shape of the molecule can be accounted for the shape factor $f_{\mathrm{s}}$ that was developed by Perrin: ${ }^{[100]}$

For prolate ellipsoids:

$$
f_{\mathrm{s}}=\frac{\sqrt{1-\left(\frac{b}{a}\right)^{2}}}{\left(\frac{b}{a}\right)^{\frac{2}{3}} \ln \frac{1+\sqrt{1-\left(\frac{b}{a}\right)^{2}}}{\left(\frac{b}{a}\right)}}
$$

For oblate ellipsoids:

$$
f_{\mathrm{S}}=\frac{\sqrt{\left(\frac{b}{a}\right)^{2}-1}}{\left(\frac{b}{a}\right)^{\frac{2}{3}} \arctan \sqrt{\left(\frac{b}{a}\right)^{2}-1}}
$$

where $a$ is the major and $b$ is the minor semiaxis of an ellipsoidal molecule. The dependence of $a / b$ on $f_{\mathrm{s}}$ is demonstrated in Fig. 1-19b. 
a)

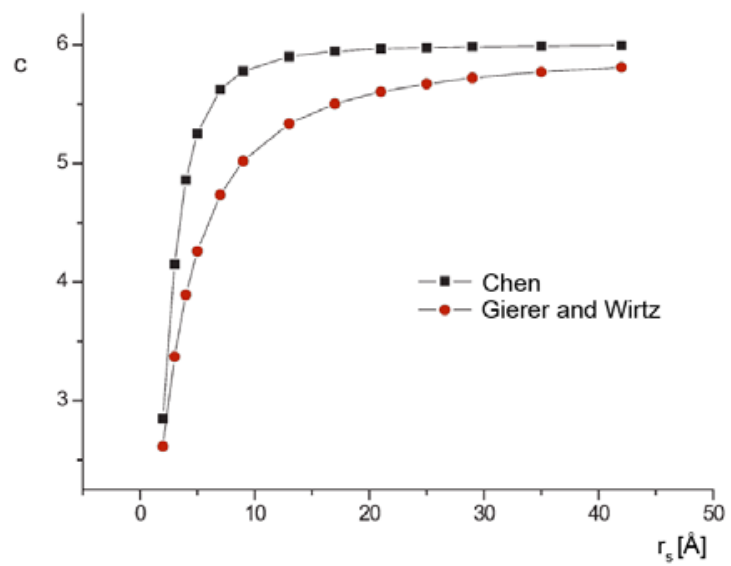

b)

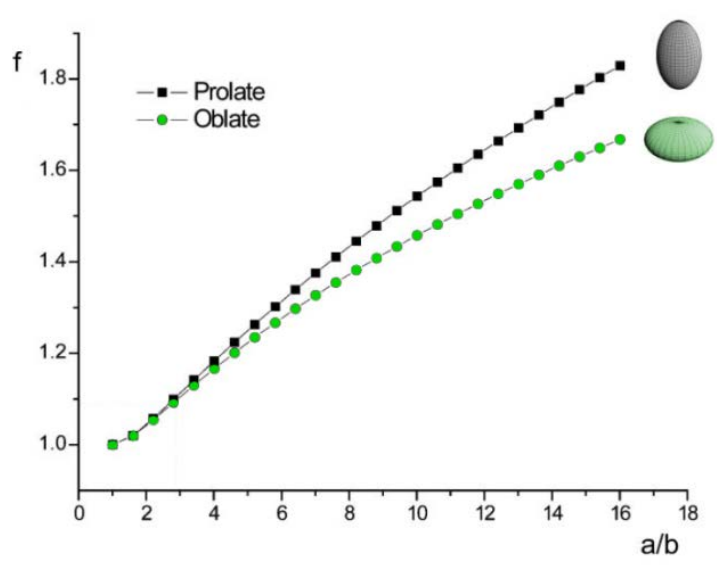

Fig. 1-19. a) Dependence of the size factor $c$ on the hydrodynamic radius of the solute $r_{\mathrm{s}}$ using methylene chloride as solvent. According to Chen as well as to Gierer and Wirtz the size coefficient $c$ tend to the value 6 with $r_{\mathrm{s}}$ values greater than $40 \AA$. b) Dependence of the ratio a/b on the shape factor $f_{\mathrm{s}}$ for prolate and oblate ellipsoidal molecules. ${ }^{[79 c]}$

Both, the Gierer-Wirtz as well the Chen modification assume the knowledge of the solvent radius $r_{\text {solv. }}$ Evans et al. modified the Gierer-Wirtz Eqn. (13) by using the effective solvent density $\rho_{\text {eff }}$ instead of the solvent radius $r_{\text {solv }}$ assuming that all small molecules have the same effective density, the same shape, solvation and flexibility. ${ }^{[101]}$ The estimation of $D$ is therefore given as:

$$
D=\frac{k_{\mathrm{b}} T\left(\frac{3 \alpha}{2}+\frac{1}{1+\alpha}\right)}{6 \pi \eta\left(\frac{3 M W_{\mathrm{s}}}{4 \pi \rho_{\mathrm{eff}} N_{\mathrm{A}}}\right)^{\frac{1}{3}}}, \text { with } \alpha=\left(\frac{M W_{\text {solv }}}{M W_{\mathrm{s}}}\right)^{\frac{1}{3}}
$$

Where $\eta$ is the viscosity, $M W_{\text {solv }}$ the molecular weight of the solvent and $M W_{\mathrm{s}}$ the molecular weight of the solute. The authors showed also that the MW-prediction of small molecules, like e.g. toluene, anthracene and cholesteryl acetate, using the unmodified Stokes-Einstein equation (9) produced an average root mean square error of RMS $=45 \%$. The application of Evan's method increases the accuracy dramatically with an average error of RMS $=15 \%$ (Tab. $1-1$ see vide infra $).{ }^{[101]}$ 


\subsubsection{Molecular Weights Derived from a Power Law}

Besides the above mentioned Stokes-Einstein-modifications, especially the empirically derived power law is probably one of the most powerful classes of relations which correlate the MW and the diffusion coefficient according to:

$$
D=K \cdot M W^{\alpha}
$$

where $D$ is the diffusion coefficient, $M W$ the molecular weight of the solute and $K$ and $\alpha$ are compound dependent constants. $\alpha$ is often related to the Flory exponent that comes from the fractal theory and can be described as a measure of compactness of a molecular shape. A Flory exponent of $-\alpha=0.33$ notes that the space is totally filled and no holes are left. On the other hand a Flory exponent of $-\alpha=1$ means that the molecule is completely one-dimensional and extends linearly like a rigid rod. ${ }^{[81 a]}$ A double logarithmic plot of the diffusion coefficients from appropriate standards against their MWs leads to a linear calibration plot (Fig. 1-20) that can be used to calculate molecular masses from diffusion coefficients.

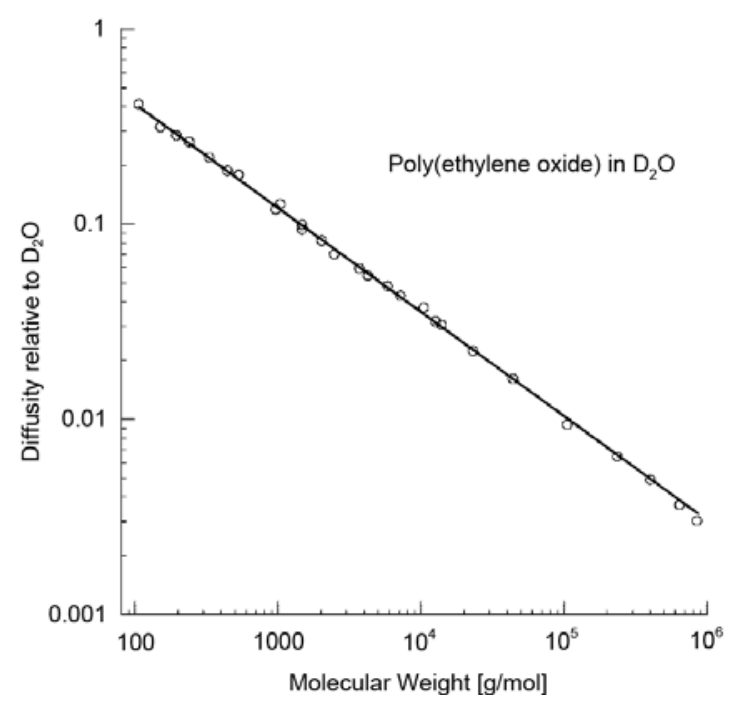

Fig. 1-20. A plot of diffusion vs. MW for a series of poly(ethylene oxide) samples in $\mathrm{D}_{2} \mathrm{O}$ provides a linear relationship. ${ }^{[81 \mathrm{a}]}$

This power law gives good results but is restricted to a specific class of compounds. ${ }^{[81 a]}$ Especially the polymer community has applied this power law to estimate the MW distribution of polymer solutions such as globular proteins, ${ }^{[81 \mathrm{a}]}$ oligosaccharides, ${ }^{[81 \mathrm{~b}]}$ polyethyleneoxides $^{[81 c]}$ and denatured peptides ${ }^{[102]}$ in various solvents. ${ }^{[6]}$ In 2007 Crutchfield and Harris showed that even small molecules correlate to the power law. ${ }^{[103]}$ In their work 
they illustrated that using the power law a relatively good MW-determination was possible with an average error of $11 \%$ (Tab. 1-1). Unfortunately, the maximum error was still very high $( \pm 35 \%){ }^{[103]}$

Tab. 1-1. RMS errors for MW-determination of small molecules $(<1100 \mathrm{~g} / \mathrm{mol})$ by different methods.

\begin{tabular}{lc}
\hline Method & RMS error / \% \\
\hline Stokes-Einstein $^{[101]}$ & 45 \\
Gierer-Wirtz $^{[101]}$ & 28 \\
Chen $^{[101]}$ & 18 \\
Evans $^{[101]}$ & 15 \\
Crutchfield $^{[103]}$ & 11
\end{tabular}

In diffusion measurements the absolute diffusion coefficient is affected by various sources of errors like e.g. diversities in temperature, fluctuation, convection, viscosity and concentration effects. In addition NMR device constants like e.g. gradient strength and pulse duration influence the absolute diffusion coefficient. To overcome this problem Williard et al. introduced several internal references (at least 3) with known MWs to the analyte sample. ${ }^{[104]}$ Since all of them experience the same physical environment the above mentioned distortions on the diffusion coefficient vanish for this specific NMR sample. By plotting the diffusion coefficients of the internal references against their MWs, an internal calibration curve (ICC) can be derived, which can be used to calculate the MW of the analyte. Using the so called "Diffusion Coefficient-Formula Weight (D-FW) Analysis" a few authors were able to characterize several organometallic compounds in solution. ${ }^{[105]}$ A great review on the practical use of this method is given in reference [77]. Unfortunately the ICC-method has some important disadvantages: on the one hand the ICC employs just a few references (mostly 3 ) and is often based on a small molecular weight distribution. On the other hand each ICC is only useful for one NMR sample. Another disadvantage is that all of the internal references have to fulfil several key factors:

(a) They should be inert to the analyte in solution.

(b) Their NMR signals should not overlap with other components.

(c) The internal references should have no coordinating ability to the analyte.

(d) All references should be well soluble in the solvent.

(e) The internal references should have a well set molecular weight distribution. 
Therefore, it is a big challenge to choose the appropriate internal references, because often at least one of the above-mentioned exigencies would not be met.

Since the authors did not take the shape of the references and the analyte into account, the accuracy the D-FW analysis is in the same range of Crutchfield's method with a maximum error of approximately $\pm 35 \%$. For example the MW prediction of acetone- $d_{6}$ gives good results $\left(M W_{\text {calc }}\left(\right.\right.$ actetone- $\left.d_{6}\right)=64 \mathrm{~g} / \mathrm{mol}, M W_{\text {det }}\left(\right.$ actetone- $\left.d_{6}\right)=67 \mathrm{~g} / \mathrm{mol}, M W_{\text {err }}$ (actetone$\left.d_{6}\right)=5 \%$ ) but that of the very flat and expended molecule chrysene produces an error of $-35 \%\left[M W_{\text {calc }}\left(\right.\right.$ chrysene- $\left.d_{12}\right)=240 \mathrm{~g} / \mathrm{mol}, M W_{\text {det }}\left(\right.$ chrysene- $\left.d_{12}\right)=158 \mathrm{~g} / \mathrm{mol}, M W_{\text {err }}($ chrysene- $\left.\left.d_{12}\right)=-35 \%\right]^{[104 \mathrm{a}]}$

\subsection{Scope of this Thesis}

The aggregation and solvation numbers of organometallics and organoamides play a dominant role in reaction mechanisms and product forming. Therefore, the knowledge of reactive aggregates especially in solution where they operate is a prerequisite to understand how these molecules react and why they yield which products. Diffusion measurements and related molecular weight (MW) determinations proved to be very helpful tools for identifying reactive intermediates in solution. However, the available methods require either several mathematical corrections or the addition of multiple internal standards. However, especially for small molecules these methods provide a significant error in MW determination.

The main scope of this thesis was the development of a straight forward methodology which determines MWs from diffusion coefficients with viable accuracy.

The "original" Stokes-Einstein equation (9) has been modified by various correction factors. These take for example the size of the solute in respect to the solvent and the shape of the solute into account. In comparison, for power law derived MW determinations the analytes have been divided into specific classes of compounds, like e.g. proteins, polymers of glycosides or poly(ethylene oxides) and the class of "small molecules" with MWs $<1100 \mathrm{~g} / \mathrm{mol}$. All of them show a linear relationship between MW and diffusion coefficient. However, the MW determination of small molecules sill results in huge maximum errors of approximately $\pm 35 \%$. Taking the shape and the density of small molecules into account could lead to improved accuracies and smaller errors. In addition, 
the D-FW analysis pioneered by $L i$ and Williard et al. is directed on multiple internal references which might interfere with the analyte. This is why the method is restricted to a handful internal references and many analytes cannot be examined due to signal overlap problems. Therefore, it would be highly beneficial if only one internal reference would be sufficient for accurate MW determinations. Furthermore, there are no investigations concerning the impact of molecule density and the absolute temperature on power law derived MW predictions.

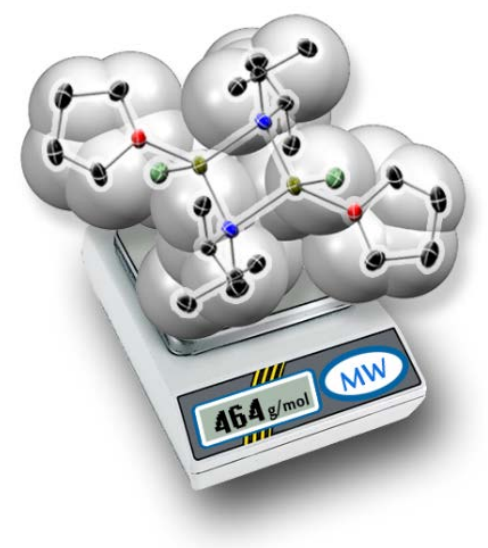

Fig. 1-21. Illustration: MW determination of dimeric Hauser base $[7 \cdot \mathrm{THF}]_{2}$ in THF solution.

Finally, the new developed MW determination methodology should be used to characterize the solution structure of prominent organometallics like e.g. LDA in toluene solution or Hauser bases (Fig. 1-21) and their Turbo-analogues in THF solution, whose solution structures were still unclear due to the absence of appropriate analytical methods. 


\section{RESULTS AND DISCUSSION}

\subsection{Development of the DOSY-External Calibration Curve MW-Determination Methodology ${ }^{8}$}

\subsubsection{Application of a Normalized Reference System with Fixed Diffusion Coefficients}

Like already mentioned in the introduction, absolute diffusion coefficients are affected by various sources of errors like e.g. diversities in temperature, fluctuation, convection, viscosity and concentration effects. Additionally the NMR-device properties and constants like gradient strength and pulse duration have an influence on the absolute diffusion coefficient (Fig. 2-1). This is why the latter can only be compared under ceteris paribus conditions. To overcome the complications of these effects and to enable tabulated diffusion coefficients, the use of an internal standard is necessary. Those standards provide more resilient diffusion coefficients for any changes in the NMR sample. A ratio, often termed as relative diffusivity $D_{\text {rel }}$ is defined as:

$$
D_{\text {rel }}=\frac{D_{\mathrm{x}}}{D_{\text {ref }}}
$$

where ref and $\mathrm{x}$ correspond to the reference and analyte, respectively. This approach reduces the impact of viscosity, temperature, NMR-device properties and provides more robust data. Besides the abovementioned advantages this method has a disadvantage. Eqn. (19) produces relative diffusion values that always depend on the one reference molecule it has been referenced for. This reference has no strict or defined diffusion value. Therefore it would be more advantageous to employ relative diffusion coefficients with fixed diffusion values. Empirically derived results showed that logarithmic diffusion values are connected approximately linearly, independent of gradient and magnetic field strength, gyromagnetic ratio, gradient pulse duration, and changes in temperature or viscosity.

\footnotetext{
${ }^{8}$ A revised version of my publication: R. Neufeld, D. Stalke, Chem. Sci. 2015, 6, 3354-3364.
} 
That is why the linear Eqn. (20) was empirically derived:

$$
\log D_{\mathrm{x}, \text { norm }}=\log D_{\text {ref,fix }}-\log D_{\text {ref }}+\log D_{\mathrm{x}}
$$

Where $\log D_{\text {reffix }}$ is the fixed value of the reference $\log D_{\text {ref }}$ the measured diffusion coefficient of the reference, $\log D_{\mathrm{x}}$ the diffusion coefficient of analyte $\mathrm{x}$ and $\log D_{\mathrm{x}, \text { norm }}$ the relative diffusion value of the analyte $\mathrm{x}$ normalized to the reference. Eqn. (20) ensures that all molecules get a normalized diffusion coefficient relative to the internal reference. For diffusion measurements for all TOL- $d_{8}$ solvates adamantane (ADAM) and for all THF- $d_{8}$ solvates 2,2,3,3-tetramethylbutane (TMB) was used as internal references (Table 2-1).

Table 2-1. $\log D_{\text {ref,fix }}$ values of the used internal references.

\begin{tabular}{cc}
\hline internal reference & $\log D_{\text {reffix }}{ }^{\text {a) }}$ \\
\hline ADAM in TOL- $d_{8}{ }^{\text {b) }}$ & -8.8454 \\
TMB in THF- $d_{8}{ }^{c)}$ & -8.7749
\end{tabular}

a) Each diffusion coefficient was estimated by using the middle $\log D$ value of 15 DOSY measurements of $15 \mathrm{mM}$ solutions at $25^{\circ} \mathrm{C}$.

b) ADAM has two signals in the ${ }^{1} \mathrm{H}-\mathrm{NMR}$-spectrum. For determining the diffusion coefficient, the signal of the $-\mathrm{CH}_{2}$ groups with the highest intensity was used.

c) The chemical shift of one ADAM signal is very close to the THF- $d_{7}$ signal at $1.73 \mathrm{ppm}$. Therefore TMB was used as internal reference for all THF- $d_{8}$ solvates.

The validity of Eqn. (20) was proved by measuring DOSY spectra of 18 model compounds on two different NMR spectrometer devices, where one spectrometer had calibrated and the other uncalibrated gradients. Although differences in gradient calibration, temperature and concentration automatically give deviations in absolute diffusion (Fig. 2-1), Eqn. (20) provides excellent results for the normalized diffusion coefficient $\log D_{\mathrm{x}, \text { norm }}$ independent of the used NMR spectrometer with an average standard deviation of only $\sigma\left(\log D_{\mathrm{x}, \text { norm }}\right)=$ 0.003 in TOL- $d_{8}$ and 0.002 in THF- $d_{8}$ (A-Table land A-Table 2 in the appendix). ${ }^{9}$ This experiment demonstrates the robustness of Eqn. (20) and the normalized diffusion coefficients.

\footnotetext{
${ }^{9}$ Derived from 18 independent measurements of 18 molecules with MWs ranging from $70 \mathrm{~g} / \mathrm{mol}$ (cyclopentane) to $623 \mathrm{~g} / \mathrm{mol}$ (BINAP).
} 
a)

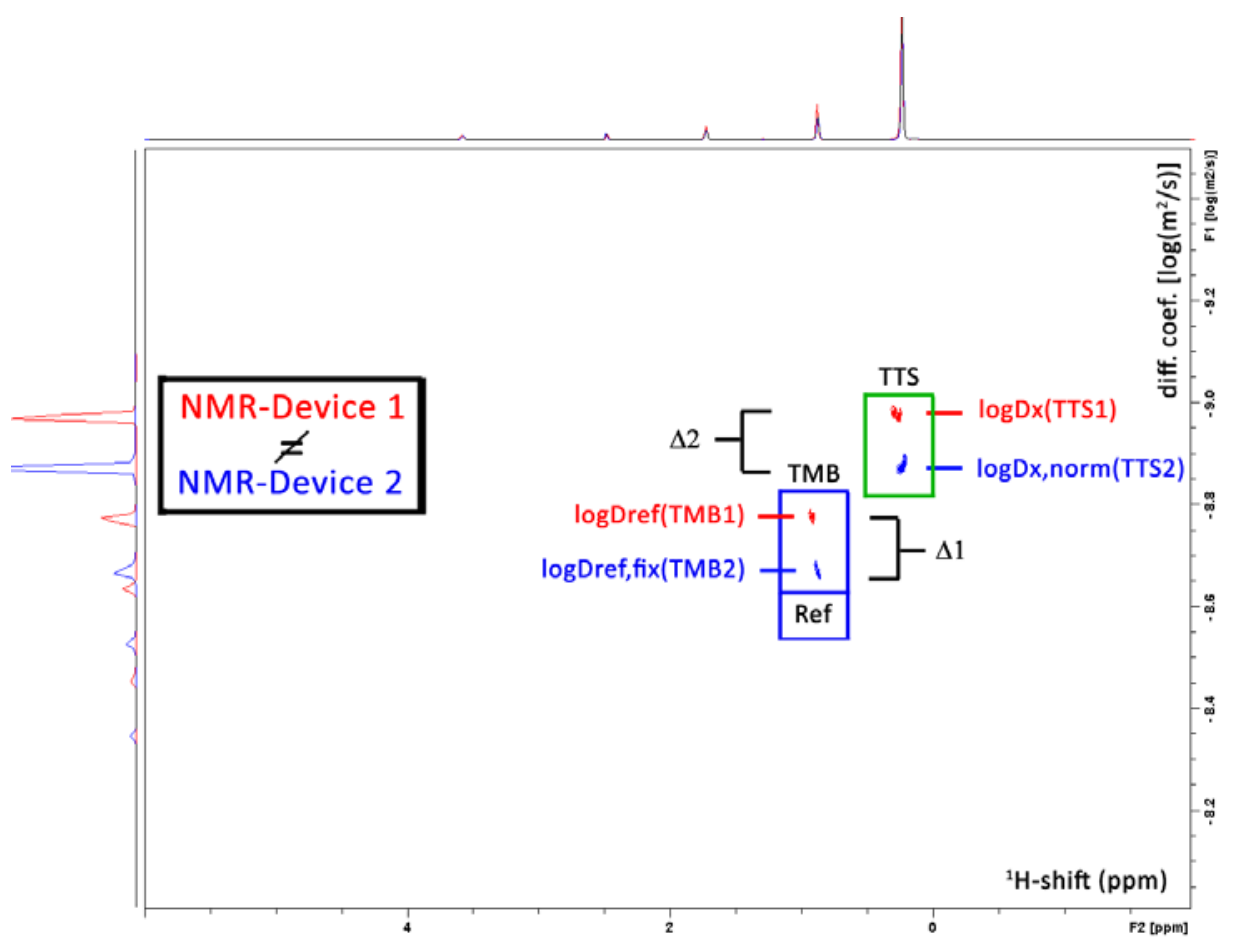

b)

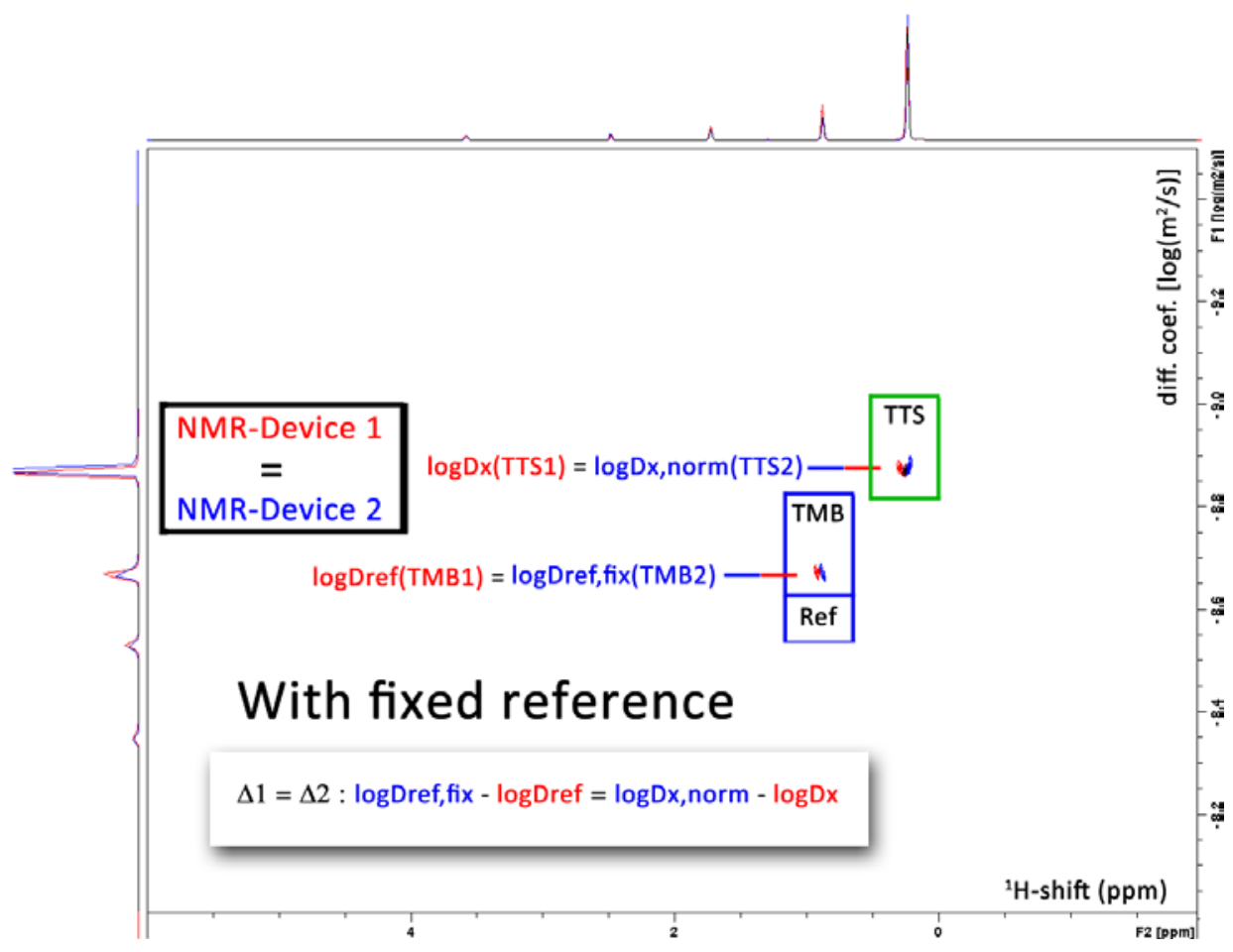

Fig. 2-1. Superposition plot of two DOSY spectra measured on two different NMR devices (red and blue). a) The absolute diffusion coefficients of $\mathrm{Si}\left(\mathrm{SiMe}_{3}\right)_{4}$ (TTS) are uneven $\log D(\mathrm{TTS} 1) \neq \log D(\mathrm{TTS} 2)$ and $\log D(\mathrm{TMB} 1) \neq \log D(\mathrm{TMB} 2)$ due to different gradient calibrations in the NMR devices and e.g. diversity in viscosity and/or temperature. b) The signal of the references has been shifted to a fixed value and the signals of TTS have been moved by the same increment of $\Delta 1=\Delta 2$. With that referencing method it is possible to obtain the same diffusion values for analyte $\mathrm{x}$ independent of the used NMR device or changes in solution properties. 


\subsubsection{External Calibration Curves (ECC) and Internal References}

The D-FW-determination developed by $L i$ and Williard et al. relies on an internal calibration curve (ICC) that has been generated by a single DOSY measurement where all references are present in the same NMR sample. The calibration curves which will be presented in this work were generated by measuring 28 model compounds in independent NMR samples. This is why these calibration curves are named "external calibration curves" (ECC). These calibration curves have been plotted the common way by linearizing the power law (18) with taking the logarithm of both sides: ${ }^{[104 d]}$

$$
\log D_{\mathrm{x}, \text { norm }}=\log K+\alpha \log M W_{\text {calc }}
$$

Plotting $\log D_{\text {x.norm }}$ versus $\log M W_{\text {calc }}$ of all model molecules in one diagram gives a linear correlation but with a significant deviation especially for the very low and higher weight molecules that prevents an accurate MW-determination (Fig. 2-2a).

a)

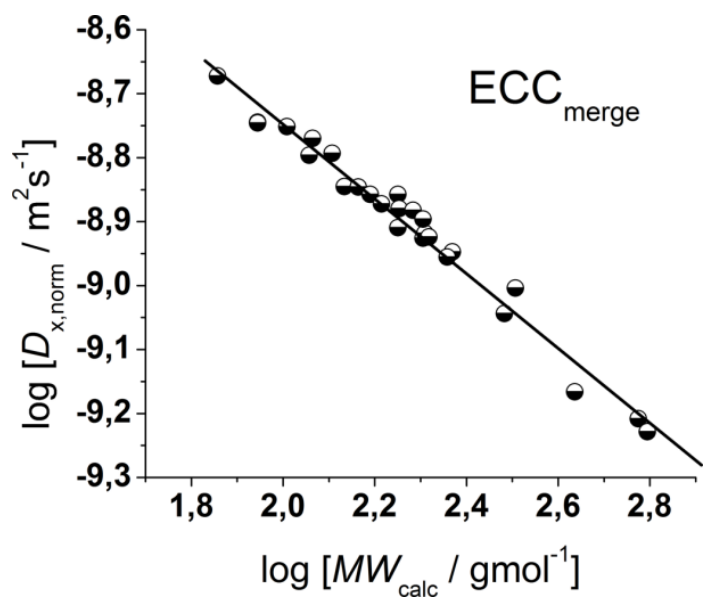

b)

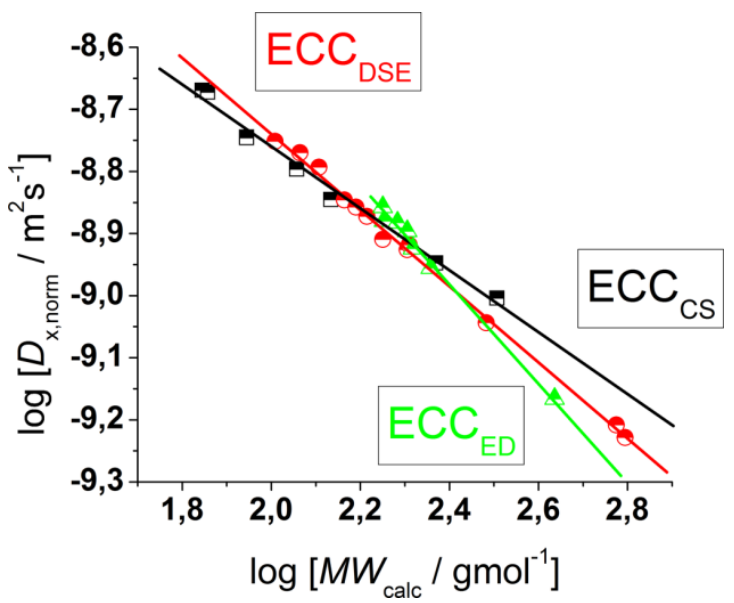

Fig. 2-2. ECC-plot: $\log D_{\mathrm{x}, \text { norm }}$ vs. $\log M W_{\text {calc }}$ of 28 model compounds in TOL- $d_{8}$. All compounds were normalized to $\log D_{\text {ref,fix }}(A D A M)=-8.8454$, see Table 2-1. a) The diffusion coefficients show a linear correlation. However, some compounds display significant deviations from a linear behavior (corr. $\mathrm{R}^{2} \leq 0.97$ ). b) Taking the shape (DSE, CS and ED) into account gives much better correlations between diffusion coefficients and MWs (corr. $\mathrm{R}^{2} \geq 0.99$ ). The same trend is observable in THF- $d_{8}$ solution.

To find a better correlation between diffusion coefficient and the MW three dimensional, shape optimized models of all model compounds were generated (A-Table 3 in the appendix). By comparing the diffusion coefficient of each molecule with its shape and compactness features, one can see a significant disparity. 
The molecules can be classified in three different types:
a) compact spheres (CS)
b) dissipated spheres and ellipsoids (DSE)
c) expanded discs (ED)

Of course the transitions between those types occur across a foggy line but there are clear systematic trends that can be rationalized. From Fig. 2-3 it is obvious, that CS have nearly the same radius in all dimensions with a high-density space like for example the compact molecules ADAM or $\mathrm{Si}\left(\mathrm{SiMe}_{3}\right)_{4}$.

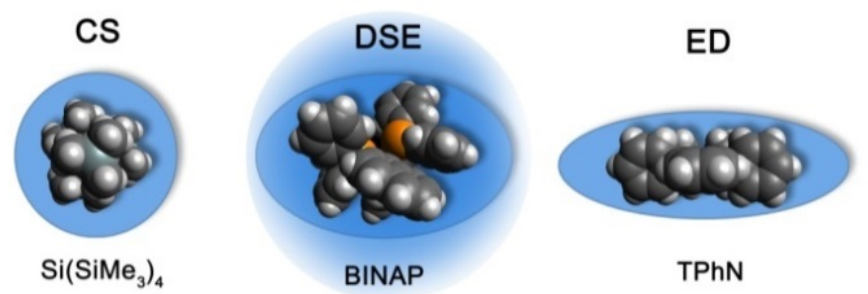

Fig. 2-3. Example molecules that were classified in the ECC as CS, DSE and ED like molecules.

However, the majority of small molecules diffuse like DSE. These are either spherical-like molecules that are less compact than CS (e.g. compounds with dative bonds like $\left[\mathrm{Li}(\mathrm{THF})_{4}\right]^{+}$) and/or ellipsoidal molecules like e. g. tetramethoxypropane or 2,2-bis (diphenylphosphino)-1,1-binaphthyl (BINAP). Small aromatic compounds like toluene, indene or naphthalene with $\mathrm{MW}<160 \mathrm{~g} / \mathrm{mol}$ diffuse also like DSE molecules. The significance of two dimensional shapes begins approximately at $\mathrm{MW}>178 \mathrm{~g} / \mathrm{mol}$. This is the region where the type of the ED begins, including molecules like anthracene $(178 \mathrm{~g} / \mathrm{mol})$ or tetraphenylnaphthalene $(\mathrm{TPhN}, 433 \mathrm{~g} / \mathrm{mol})$. Depending on these different molecular types ${ }^{10}$ the ECC plot gives excellent linear fits (Fig. 2-2b) with a small error and very high $\mathrm{R}^{2}$ values of $\mathrm{R}^{2} \approx 1$ (A-Figure 1 and A-Figure 2 in the appendix). These fits result in six different ECCs: $\mathrm{ECC}_{\mathrm{CS}}, \mathrm{ECC}_{\mathrm{DSE}}$ and $\mathrm{ECC}_{\mathrm{ED}}$ for each solvent (TOL- $d_{8}$ and THF- $d_{8}$ ). The linear fit parameters are summarized in Table 2-2. Merged calibration curves were also generated, for MW-determinations of compounds with unknown molecular shapes.

\footnotetext{
${ }^{10} \mathrm{~A}$ fourth type of molecule shape is also thinkable that should distinguish from the already mentioned ones: rod shaped molecules. However, in this Ph.D. thesis the focus is on CS, DSE and ED molecules.
} 
Table 2-2. Linear fit parameter for the four ECCs each for TOL- $d_{8}$ and THF- $d_{8}$ solutions

\begin{tabular}{ccccc}
\hline & \multicolumn{2}{c}{ TOL- $d_{8}$} & \multicolumn{2}{c}{ THF- $d_{8}$} \\
& $-\log K$ & $-\alpha$ & $-\log K$ & $-\alpha$ \\
\hline ECC $_{\mathbf{C S}}^{\mathbf{S}}$ & 7.7581 & 0.5018 & 7.7427 & 0.4943 \\
ECC $_{\mathbf{D S E}}^{\mathbf{S}}$ & 7.5197 & 0.6098 & 7.5360 & 0.5824 \\
ECC $_{\mathbf{E D}}^{\mathbf{S}}$ & 7.1008 & 0.7836 & 7.1205 & 0.7519 \\
ECC $_{\text {merge }}^{\mathbf{S}}$ & 7.4595 & 0.6318 & 7.4664 & 0.6095
\end{tabular}

When internal calibration curves (ICC) are used, then all references have to be in the same sample. The diffusion coefficients of those internal references show a linear dependency. In the above presented external calibration curves (ECC), (where each model compound has been measured with $\mathrm{ADAM}$ or TMB as single internal reference), a linear behavior is observable, too. These compounds behave as they were all measured in the same NMR sample. Therefore the idea occurred that beside ADAM and TMB, basically all model compounds could act as internal references for the ECCs, according to:

$$
\log D_{\mathrm{x}, \mathrm{norm}}=\log D_{\text {ref,fix }}^{*}
$$

Consequently DOSYs were measured with some model compounds (e.g. ADAM + $\mathrm{Si}\left(\mathrm{SiMe}_{3}\right)_{4}+$ naphthalene in TOL- $\left.d_{8}\right)$ in the same NMR sample. Every compound was used as internal reference by applying equation (22). In fact it is possible to determine accurate MWs of all compounds by using the normalized $\log D_{\mathrm{x}, \text { norm }}$ value as fixed reference (Table 2-3). The MWs can be calculated by using the ECC-parameters from Table 2-2 and following equation:

$$
M W_{\text {det }}=10^{\left(\frac{\log D_{\mathrm{x}, \mathrm{norm}-\log K}}{\alpha}\right)}
$$

And the deviation of the determined MW $\left(M W_{\text {det }}\right)$ from the calculated MW $\left(M W_{\text {calc }}\right)$ is estimated according to:

$$
M W_{\mathrm{err}}=\left[1-\frac{M W_{\mathrm{det}}}{M W_{\mathrm{calc}}}\right] \cdot 100 \%
$$

Utilizing e.g. the residual proton signal TOL- $d_{7}$ as internal reference, $\mathrm{ADAM}, \mathrm{Si}\left(\mathrm{SiMe}_{3}\right)_{4}$ and naphthalene can be determined with an average deviation of $\pm 5 \%$. Or in other words, the "real" molecular weight was missed by only $5 \%$, although a calibration curve was used which relies on the basis of many references that were not present in this NMR sample. 
Table 2-3. Mixed composition of compounds (each $15 \mathrm{~mm}$ ) in TOL- $d_{8}$ acting them self as internal reference for the DOSY-ECC-MW-determination.

\begin{tabular}{|c|c|c|c|c|c|c|c|c|c|}
\hline \multirow[b]{2}{*}{ Analyte } & \multirow[b]{2}{*}{$\begin{array}{l}M W_{\text {calc }} \\
{[\mathrm{g} / \mathrm{mol}]}\end{array}$} & \multicolumn{2}{|c|}{$\begin{array}{c}\operatorname{Ref} 1 \\
\text { TOL- } d_{7}\end{array}$} & \multicolumn{2}{|c|}{$\begin{array}{c}\text { Ref } 2 \\
\text { ADAM }\end{array}$} & \multicolumn{2}{|c|}{$\begin{array}{c}\operatorname{Ref3} \\
\mathrm{Si}\left(\mathrm{SiMe}_{3}\right)_{4}\end{array}$} & \multicolumn{2}{|c|}{$\begin{array}{c}\text { Ref } 4 \\
\text { Naphthalene }\end{array}$} \\
\hline & & $\begin{array}{c}M W_{\mathrm{det}} \\
{[\mathrm{g} / \mathrm{mol}]}\end{array}$ & $\begin{array}{c}M W_{\text {err }} \\
{[\%]}\end{array}$ & $\begin{array}{c}M W_{\mathrm{det}} \\
{[\mathrm{g} / \mathrm{mol}]}\end{array}$ & $\begin{array}{c}M W_{\text {err }} \\
{[\%]}\end{array}$ & $\begin{array}{c}M W_{\text {det }} \\
{[\mathrm{g} / \mathrm{mol}]}\end{array}$ & $\begin{array}{c}M W_{\text {err }} \\
{[\%]}\end{array}$ & $\begin{array}{c}M W_{\text {det }} \\
{[\mathrm{g} / \mathrm{mol}]}\end{array}$ & $\begin{array}{c}M W_{\text {err }} \\
{[\%]}\end{array}$ \\
\hline TOL- $d_{7}{ }^{\mathrm{b})}$ & 99 & 96 & 3 & 97 & 2 & 96 & 3 & 97 & 2 \\
\hline $\mathrm{ADAM}^{\mathrm{a})}$ & 136 & 144 & -6 & 147 & -8 & 144 & -6 & 145 & -7 \\
\hline $\mathrm{Si}\left(\mathrm{SiMe}_{3}\right)_{4}{ }^{\text {a) }}$ & 321 & 304 & 5 & 309 & 4 & 303 & 5 & 305 & 5 \\
\hline Naphthalene ${ }^{b)}$ & 128 & 122 & 5 & 124 & 3 & 122 & 5 & 122 & 5 \\
\hline
\end{tabular}

a) $\left.\operatorname{ECC}_{C S}^{T O L}, b\right) \operatorname{ECC}_{D S E}^{T O L}$ were used to calculate the MW.

With the ECC-method it is possible to simulate a bench of internal references by adding just one of them to the NMR sample. All 28 compounds behave like they were all measured in the same NMR sample. Inert compounds that are suitable to act as internal reference and their $\log D_{\text {ref,fix }}^{*}$ values are summarized in Table 2-4. In the next chapter the use of the residual solvent signal of TOL- $d_{8}$ and THF- $d_{8}$ as internal reference is examined in detail.

Table 2-4. Overview of all ECC-adapted inert references that fulfill the requirement of internal references for $15 \mathrm{mM}$ solutions.

\begin{tabular}{cccc}
\hline $\begin{array}{c}M W_{\text {calc }} \\
{[\mathrm{g} / \mathrm{mol}]}\end{array}$ & Compound $^{\text {a) }}$ & $\begin{array}{c}\text { TOL- } d_{8} \\
\log D_{\text {ref,fix }}^{*}\end{array}$ & $\begin{array}{c}\text { THF- } d_{8} \\
\log D_{\text {ref,fix }}^{*}\end{array}$ \\
\hline 70 & Cyclopentane & -8.6694 & -8.6437 \\
79 & THF- $d_{7}$ b,f) & -- & -8.6335 \\
88 & TMS & -8.7445 & -8.7018 \\
92 & TOL & -- & -8.6742 \\
99 & TOL- d $_{7}$ d,f) & -8.7289 & -- \\
114 & TMB & -8.7963 & $-8.7749^{\text {c) }}$ \\
116 & Indene & -8.7698 & -8.7325 \\
128 & Naphthalene & -8.7932 & -8.7461 \\
136 & ADAM $)$ & $-8.8454^{\mathrm{c})}$ & -- \\
178 & Diphenylacetylene & -8.9095 & -8.8535 \\
178 & Anthracene & -8.8574 & -8.8129 \\
192 & 9-Methylanthracene & -8.8824 & -8.8440 \\
202 & Pyrene & -8.8960 & -8.8457 \\
204 & 1-Phenylnaphthalene & -8.9184 & -8.8812 \\
228 & Triphenylene & -8.9552 & -8.8869 \\
321 & Si(SiMe $)_{3}$ & -9.0038 & -8.9773 \\
433 & Tetraphenylnaphthalene & -9.1660 & -9.1054
\end{tabular}


a) When a compound had more than one ${ }^{1} \mathrm{H}$ signal, the average diffusion coefficient was used.

b) Due to the very high access of the solvent, the signal of THF- $d_{7}$ can be used as internal reference, but a higher $M W_{\text {det }}$ error can occur, when the solvent is coordinating to e.g. a metal.

c) The "original" $\log D_{\text {ref,fix }}$ values that were used for all ECCs.

d) A the middle diffusion coefficients of the three aromatic protons was calculated. The final diffusion coefficient was calculated by middling this value with the diffusion coefficient of the methyl group at $1.73 \mathrm{ppm}$.

e) $\quad \mathrm{ADAM}$ has two signals in the ${ }^{1} \mathrm{H}$-spectrum. For determining the diffusion coefficient, the signal of the $-\mathrm{CH}_{2}$ groups with the highest intensity was used.

f) The residual solvent signal of TOL- $d_{8}$ and THF- $d_{8}$ that arises from the proton of isotopomers containing one less deuterium atom than the perdeuterated solvent ${ }^{[106]}$ are referred as TOL- $d_{7}$ and THF- $d_{7}$.

\subsubsection{Quality of TOL- $d_{7}$ and THF- $d_{7}$ as Internal Reference}

To compare the quality of the ECCs, MWs of all model compounds were calculated by using the $\log D_{\text {ref,fix }}^{*}$ values of TOL- $d_{7}$ and THF- $d_{7}$ as internal references (Table 2-4). The MWs of the model compounds were determined by using the appropriate ECCs. The MW of cyclopentane (Fig. 2-4, very left point, $M W_{\text {err }}=7 \%$ ) was e.g. determined by using $\mathrm{ECC}_{\mathrm{CS}}$

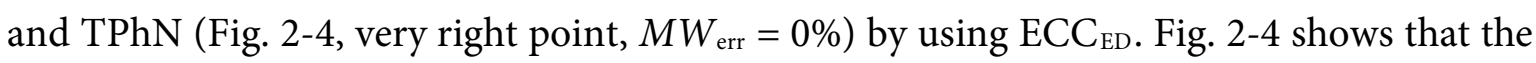
quality of MW-determination is reference-independent. It does not matter if ADAM/TMB or TOL- $d_{7} /$ THF- $d_{7}$ were used as internal reference. Both give excellent MW-predictions with a standard deviation of only $\sigma=4 \%$. The maximum error in both solvents is $M W_{\text {err }} \leq \pm 9 \%$. Therefore it can be postulated that this is the maximum resolution of this DOSY-ECC-MW-determination method.

a)

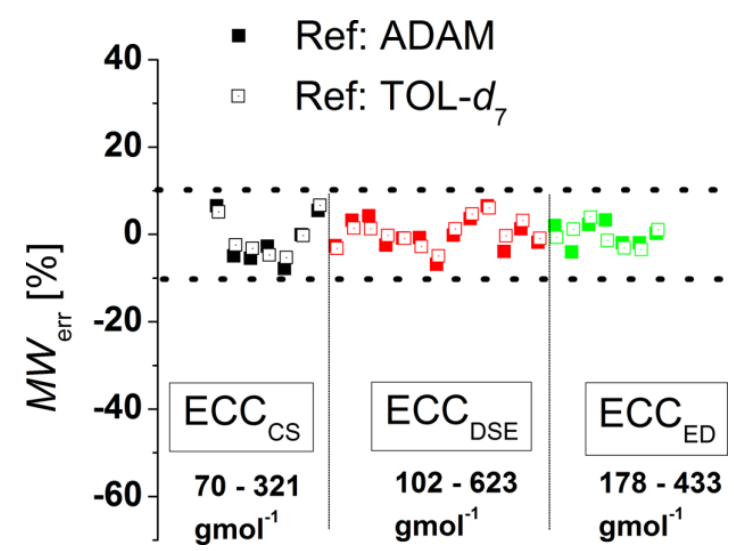

b)

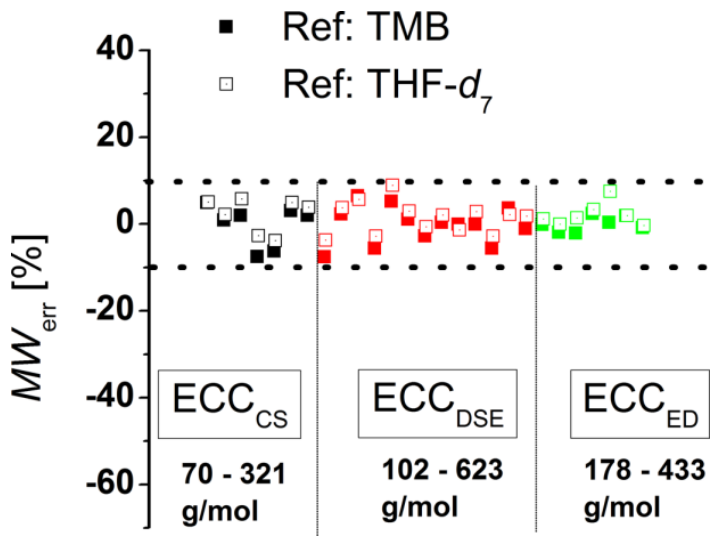

Fig. 2-4. Using a) ADAM or TOL- $d_{7}$ and b) TMB or THF- $d_{7}$ as internal reference (15 mM) gives a good MW-determination with a standard deviation of only $4 \%$. 


\subsubsection{Influence of Shape}

There are several modifications of the Stokes-Einstein equation which take the molecule's shape into account (by adding e.g. correction factors). ${ }^{[79,99,101]}$ The power law derived MWdeterminations distinguish mostly between compound classes, ${ }^{[81 a]} \operatorname{larg} \mathrm{e}^{[107]}$ and small molecules, ${ }^{[103]}$ but not directly between different shapes within those molecular classes. In this chapter it will be demonstrated that the accuracy of the power law derived MWprediction is highly affected, also for small molecules, by the analyte's shape. To validate this issue external calibration curves $\mathrm{ECC}_{\mathrm{ED}}, \mathrm{ECC}_{\mathrm{DSE}}$ and $\mathrm{ECC}_{\mathrm{ED}}$ were used for MWdetermination of all molecules without considering their true shape (Fig. 2-5). When for example the $\mathrm{ECC}_{\mathrm{CS}}$ (that is for compact spherical molecules) is used on expanded disc like molecules, the determined MW will have a massive error especially for big molecules (Fig. $2-5 \mathrm{~A})$.

a)

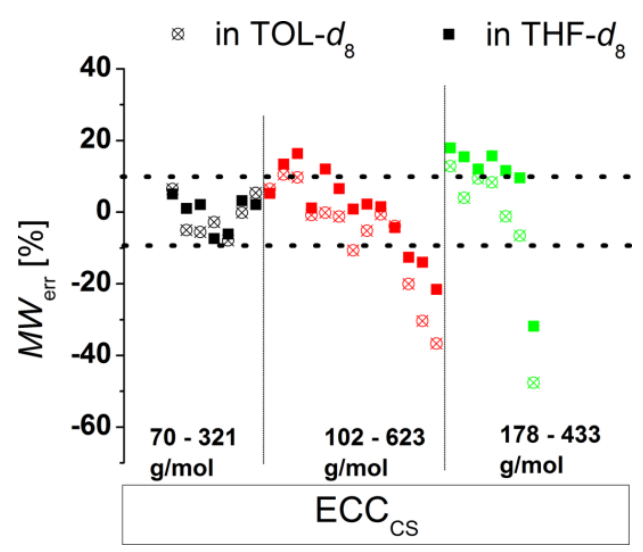

c)

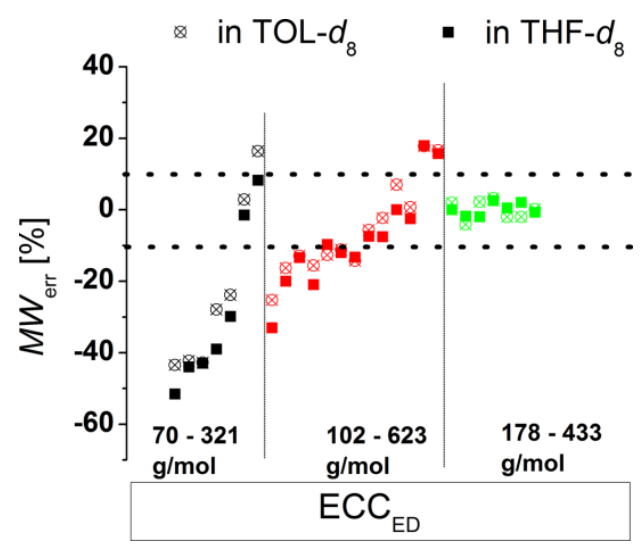

b)

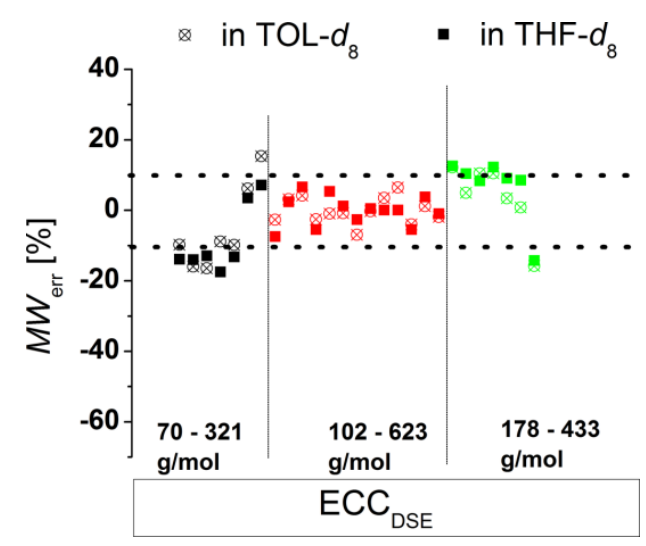

d)

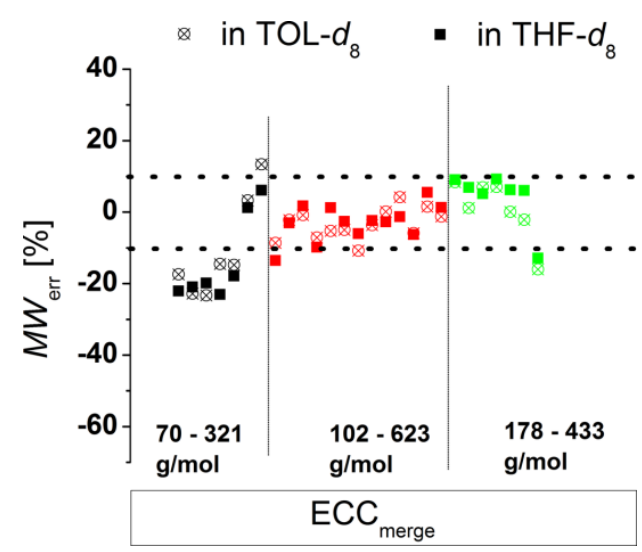

Fig. 2-5. Using exclusively a) ECC $C S$, b) ECC $C_{D S E}$ c) ECC $E_{E D}$ and d) $E C_{\text {merge }}$ on all model compounds (15 mM) in TOL- $d_{8}$ and THF- $d_{8}$. E.g. the MW-determination of TPhN (an expanded flat disc, $M W_{\text {calc }}=433 \mathrm{~g} / \mathrm{mol}$ ) in TOL- $d_{8}$ with spherically references $\left(\mathrm{ECC}_{\mathrm{CS}}\right.$ ) would produce a $\mathrm{MW}$ of $M W_{\mathrm{det}}=639 \mathrm{~g} / \mathrm{mol}$ with an error of $M W_{\text {err }}=48 \%$. This is why it is very important to use appropriate references with right shapes for accurate MW-determinations. 
Anticipating for example TPhN (an expanded flat disc, $433 \mathrm{~g} / \mathrm{mol}$ ) in TOL- $d_{8}$ to be spherical would produce a $\mathrm{MW}$ of $M W_{\text {det }}=639 \mathrm{~g} / \mathrm{mol}$ that is a $48 \%$ overestimated mass (Fig. 2-5A). Using e.g. the $\mathrm{ECC}_{\mathrm{ED}}$ for non-oblate molecules would produce especially for small molecules with $\mathrm{MW}_{\text {calc }}<170 \mathrm{~g} / \mathrm{mol}$ a large error (Fig. 2-5C). The merged calibration curve $\mathrm{ECC}_{\text {merge }}$ (Fig. 2-5D) determines $\mathrm{MWs}$ in a range of $M W_{\text {err }}= \pm 23 \%$. However, the deviation is much smaller in the region of approximately $120-200 \mathrm{~g} / \mathrm{mol}$. On one hand that means that in this MW-region all molecules diffuse more or less independently from their shape. On the other hand the MW-determination (and the self-diffusion) of molecules that are outside that region, is increasingly influenced by the analyte's shape. Using the wrong calibration curve (or for example wrong references for an ICC) can produce highly erroneous MW-values. This is the reason why other calibration curves ${ }^{[101,103,108]}$ that correlate a bundle of different molecules without considering the right shape, lead to bigger deviations from the correct MWs in the range of $M W_{\text {err }}= \pm 30 \%$. By taking the correct shape into account it is possible to determine more accurate MWs with a deviation of $M W_{\text {err }} \leq \pm 9 \%$ (A-Table 6 and A-Table 7 in the appendix).

\subsubsection{Influence of Concentration}

All above mentioned ECCs were derived from diluted solutions (15 mM). It was important to validate how good the ECC-MW-determination works, when the concentration is much higher than $15 \mathrm{mM}$. Therefore DOSYs of some model compounds have been measured at significantly higher concentrations of $240 \mathrm{mM}$ (reference and analyte each $120 \mathrm{~mm}$ ). The results are shown in Fig. 2-6.

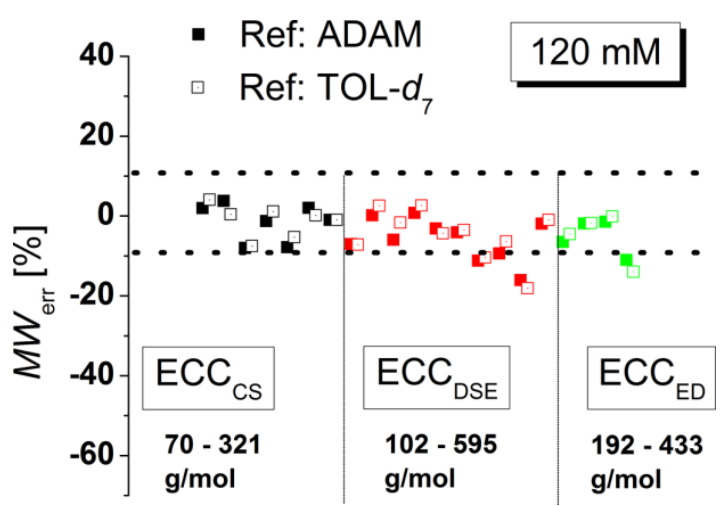

Fig. 2-6. DOSY-ECC-MW-determination of some model compounds at a concentration of $120 \mathrm{mM}$ at $25^{\circ} \mathrm{C}$ in TOL- $d_{8}$. ADAM was used in equimolar concentration as internal reference. 
The Stokes-Einstein equation is only valid for infinite diluted solutions. Therefore the error should be bigger with high concentrated solutions. However, the average deviation of the estimated MW is only a little higher and most of the compounds are still in the $\pm 9 \%$ range. The biggest error arises probably due to intermolecular interactions that result in higher estimated MWs. Especially $\pi$-interactions of big aromatic systems, at high concentrations could significantly increase the error of estimated MWs (A-Table 8 in the appendix). Anyway, all compounds without aromatic rings have been determined within $\pm 9 \%$ deviation. The power law seems to be valid even for higher concentrations, if inter- or intramolecular interactions can be excluded. At lower temperatures inter- or intramolecular interactions increase. Therefore the error of MW-determination, especially for huge aromatic systems should also increase with high concentrations. This is why for low temperature measurements diluted samples should be used. Thanks to the internal reference, the ECC-MW-determination.

\subsubsection{Influence of Temperature}

According to the Stokes-Einstein equation the self-diffusion should be inversely proportional to the viscosity. Indeed, increasing the solvent viscosity by cooling the NMR sample from room temperature to $-75^{\circ} \mathrm{C}$ leads to an increase of the diffusion coefficient by almost two magnitudes! Thanks to the internal reference, the ECC-MW-determination of $\mathrm{Si}\left(\mathrm{SiMe}_{3}\right)_{4}$ (TTS, $321 \mathrm{~g} / \mathrm{mol}$ ) gives in the full range of $-75^{\circ} \mathrm{C}$ to $+100^{\circ} \mathrm{C}$ still good results (Fig. 2-7).

a)

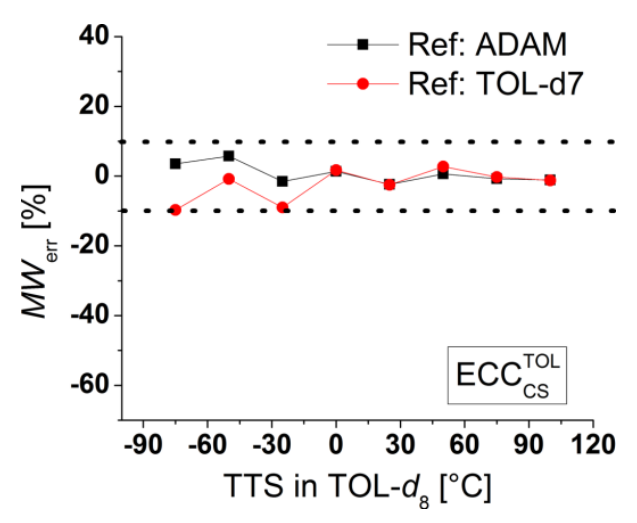

b)

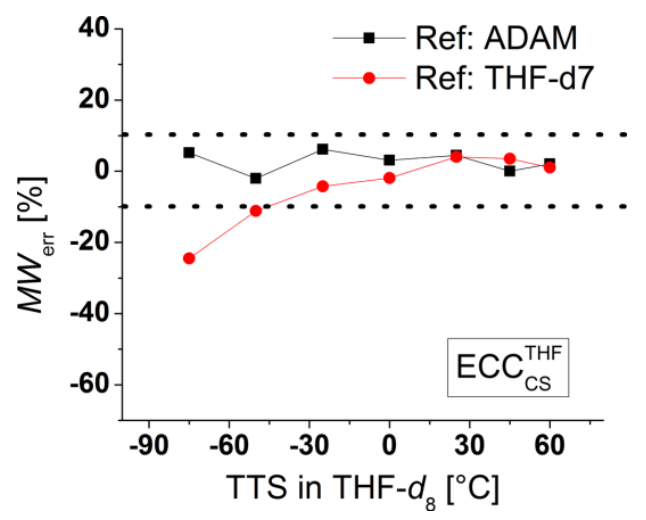

Fig. 2-7. DOSY-ECC-MW-determination of $\mathrm{Si}\left(\mathrm{SiMe}_{3}\right)_{4}$ (TTS, $15 \mathrm{mM}$ ) in a) TOL- $d_{8}$ and in b) THF- $d_{8}$ at different temperatures. Thanks to the internal reference, the ECC-MW-determination of TTS gives in the full temperature range good results. However, below $-50^{\circ} \mathrm{C}$ the use of the residual THF proton signal THF- $d_{7}$ can be problematically, due to hydrogen bonding with residual water molecules. 
The internal reference is able to compensate for viscosity changes in the solvent (see also A-Table 9 in the appendix). Notably, the MW-determination is still possible at temperatures close to the boiling point of the solvent. This would give the opportunity to observe species that are forming during reactions at elevated temperatures. Furthermore, it is obvious that the signal of TOL- $d_{7}$ is a useful internal reference for both high and low temperatures. However, using more polar THF- $d_{7}$ below $-50^{\circ} \mathrm{C}$ can get problematically. This probably results from solvent-solvent interactions and hydrogen bonding with residual water molecules.

\subsubsection{Influence of Halides and Molar Density}

In the Stokes-Einstein equation the diffusion coefficient relies on the shape and on the hydrodynamic radius of the particle. The latter can be described by the volume of the surrounding solvent molecules, the electron cloud and also the volume of the atoms. One has to keep in mind that the volume of an atom is not proportional to its atom weigh. Especially halides, compared to their atomic radii, have very high atomic masses and therefore a high mass density. For instance a potassium cation has an ion radius of $138 \mathrm{pm}$ and an atom weight of $39 \mathrm{~g} / \mathrm{mol}$. An iodine atom has almost the same radius of $133 \mathrm{pm}$ but an atom weight of $127 \mathrm{~g} / \mathrm{mol}$ that is $320 \%$ higher than that of the $\mathrm{K}^{+}$cation. ${ }^{[109]}$ The herein presented ECCs were elaborated with references that consist of hydrocarbons with some heteroatoms of the third period like silicon, phosphor and sulfur. Therefore especially compounds containing heavy halides will be underestimated in MW. While chlorine containing compounds are estimated with good accuracies the much denser bromides show bigger deviations from the correct MWs (Table 2-5).

Table 2-5. DOSY-ECC-MW-determination of molecules with halides.

\begin{tabular}{ccccc}
\hline Compound & $\begin{array}{c}M W_{\text {calc }} \\
{[\mathrm{g} / \mathrm{mol}]}\end{array}$ & $\begin{array}{c}M W_{\text {det }} \\
\text { a) } \\
{[\mathrm{g} / \mathrm{mol}]}\end{array}$ & $\begin{array}{c}M W_{\text {err }} \\
{[\%]}\end{array}$ & $\begin{array}{c}M D_{\mathrm{w}} / 10^{29} \\
{\left[\mathrm{~g} /\left(\mathrm{mol} \cdot \mathrm{m}^{3}\right)\right]}\end{array}$ \\
\hline 1-Hexylchloride & 120 & 117 & 2 & 5.49 \\
1-Octylchloride & 149 & 143 & 4 & 5.29 \\
1-Decylchloride & 177 & 176 & 1 & 5.13 \\
1-Propylbromide & 123 & 82 & 34 & 9.66 \\
Triphenylmethylbromide & 323 & 283 & 12 & 6.45 \\
9,10-Dibromoanthracene & 336 & 194 b) & 42 & 8.71 \\
1-Butyliodide & 184 & 102 & 45 & 11.15
\end{tabular}

a) $\mathrm{ECC}_{\mathrm{DSE}}$ and b) $\mathrm{ECC}_{\mathrm{ED}}$ was used to determine the MW 
Especially increasing halide to carbon ratio leads to bigger errors. For example triphenylmethylbromide with one bromine atom is underestimated by $12 \%$. But 9,10 dibromoanthracene with two bromides, a small carbon backbone and therefore a very high molar density is underestimated in MW by $42 \%$. These examples demonstrate that the power law depends not only on the shape of the molecules but also heavily on the molecular density.

There are more or less extensive ways to calculate the density of a molecule. However, a simple but robust equation that correlates the MW to the approximated volume of a compound would be highly beneficial. Therefore equation (25) was derived:

$$
M D_{\mathrm{W}}=\frac{M W_{\text {calc }}}{V_{\text {sph }}}, \text { with } V_{\mathrm{sph}}=\sum V_{W}=\sum_{i=1}^{n} \frac{3}{4} \pi r_{w, i}^{3}
$$

where $M D_{\mathrm{W}}$ is the molar van der Waals density, $M W_{\text {calc }}$ the calculated molecular weight of a compound, $V_{\mathrm{W}}$ the van der Waals volume and $r_{\mathrm{W}}$ the van der Waals radius of $n$ atoms. In respect to this equation the van der Waals volumes ${ }^{[110]}$ of all atoms of a compound were calculated and summed up to one single van der Waals-sphere $\left(V_{\mathrm{Sph}}\right)$ (A-Table 10 in the appendix). Of course this method is just an approximation without considering the real covalent bond-bond distances and the shape of the compounds. But the ratio between the MW and the sum of all van der Waals volumes ( $V_{\mathrm{w}}$, see Table 2-7, vide infra) leads to a 


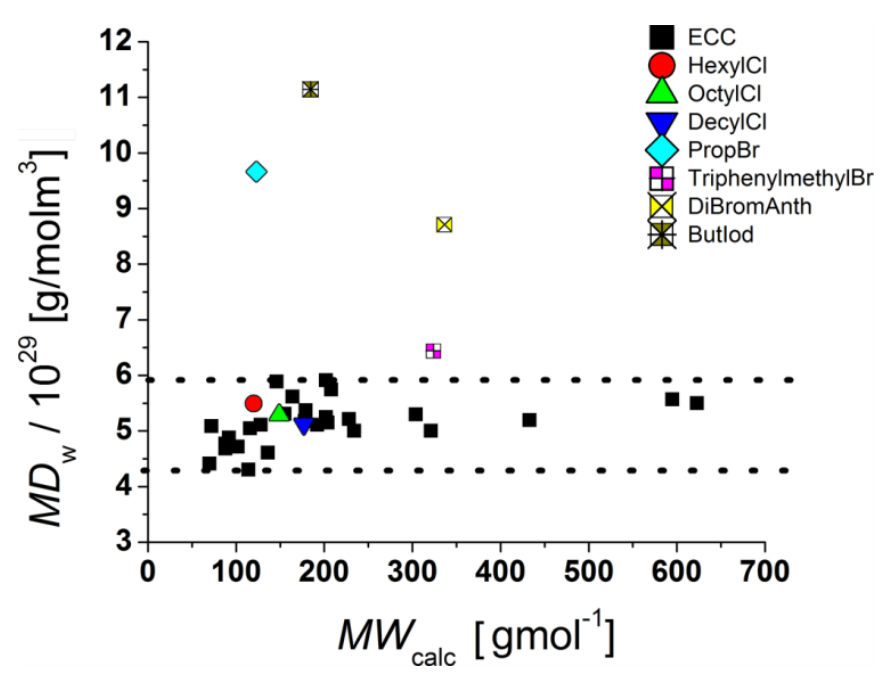

Fig. 2-8. Molar van der Waals-density $\left(M D_{\mathrm{W}}\right)$ distribution in the model compounds and molecules with heavy atoms. The ECCs presented in this thesis work well with molecules with a molar density between $4.3 \cdot 10^{29} \mathrm{~g} /\left(\mathrm{mol} \cdot \mathrm{m}^{3}\right)$ and $5.9 \cdot 10^{29} \mathrm{~g} /\left(\mathrm{mol} \cdot \mathrm{m}^{3}\right)$.

value that represents approximately a molar van der Waals density $\left(M D_{\mathrm{W}}\right)$ in a unit of $\mathrm{g} /\left(\mathrm{mol} \cdot \mathrm{m}^{3}\right)$. Plotting $M D_{\mathrm{W}}$ against $M W_{\text {calc }}$ give for all model compounds an average density distribution of around $5.2 \cdot 10^{29} \mathrm{~g} /\left(\mathrm{mol} \cdot \mathrm{m}^{3}\right)$ (Fig. 2-8). Obviously the ECCs presented in this thesis work well with molecules with a molar density between $4.3 \cdot 10^{29} \mathrm{~g} /\left(\mathrm{mol} \cdot \mathrm{m}^{3}\right)$ and $5.9 \cdot 10^{29} \mathrm{~g} /\left(\mathrm{mol} \cdot \mathrm{m}^{3}\right)$. Higher molar van der Waals densities like for example 9,10dibromoanthracene $\left[M D_{\mathrm{W}}=8.7 \cdot 10^{29} \mathrm{~g} /\left(\mathrm{mol} \cdot \mathrm{m}^{3}\right)\right.$, Table 2-5] will be underestimated and lower $M D_{\mathrm{w}}$ values will be overestimated in MW. To obtain accurate MWs for molecules with high densities it is necessary to measure new calibration curves with references of comparable molar densities and shapes. However, in order to apply a density correction on the $M W_{\text {det }}$ value of highly densed molecules a correction factor $X_{\text {corr }}$ can be calculated for each ECC (Table 2-6).

$$
X_{\text {corr }}=\frac{M W_{\text {calc }}}{M W_{\text {det }}}
$$

Table 2-6. DOSY-ECC-MW-determination of various compounds (DSE shape, $20 \mathrm{mM}$ in THF- $d_{8}$ ) with high molar van der Waals densities.

\begin{tabular}{cccccc}
\hline & Formula & $\begin{array}{c}M D_{\text {w }} \\
{\left[\mathrm{g} /\left(\mathrm{mol} \cdot \mathrm{m}^{3}\right)\right]}\end{array}$ & $\begin{array}{c}M W_{\text {calc }} \\
{[\mathrm{g} / \mathrm{mol}]}\end{array}$ & $\begin{array}{c}M W_{\text {det }} \\
{[\mathrm{g} / \mathrm{mol}]}\end{array}$ & $X_{\text {corr }}$ \\
\hline 1-Butylbromide & $\mathrm{C}_{4} \mathrm{H}_{9} \mathrm{Br}$ & $8.62 \mathrm{E}+29$ & 137 & 93 & 1.473 \\
1-Propylbromide & $\mathrm{C}_{3} \mathrm{H}_{7} \mathrm{Br}$ & $9.66 \mathrm{E}+29$ & 123 & 82 & 1.500 \\
1-Pentylbromide & $\mathrm{C}_{5} \mathrm{H}_{11} \mathrm{Br}$ & $7.92 \mathrm{E}+29$ & 151 & 106 & 1.425 \\
1-Butyliodide & $\mathrm{C}_{4} \mathrm{H}_{9} \mathrm{I}$ & $1.12 \mathrm{E}+30$ & 184 & 102 & 1.804
\end{tabular}




$\begin{array}{cccccc}\mathrm{FeCp}_{2} & \mathrm{C}_{10} \mathrm{H}_{10} \mathrm{Fe}_{2} & 6.89 \mathrm{E}+29 & 186 & 153 & 1.216 \\ \text { Triphenylmethylbromide } & \mathrm{C}_{19} \mathrm{H}_{15} \mathrm{Br} & 6.45 \mathrm{E}+29 & 323 & 290 & 1.114 \\ \text { 2-Chlorobenzoxazole } & \mathrm{C}_{7} \mathrm{H}_{4} \mathrm{ClNO} & 6.66 \mathrm{E}+29 & 146 & 124 & 1.177\end{array}$

Plotting e.g. the $X_{\text {corr }}$ factor of molecules with DSE shape (Table 2-6) against their molar van der Waals density $M D_{\mathrm{w}}$ gives a linear dependency (Fig. 2-9):

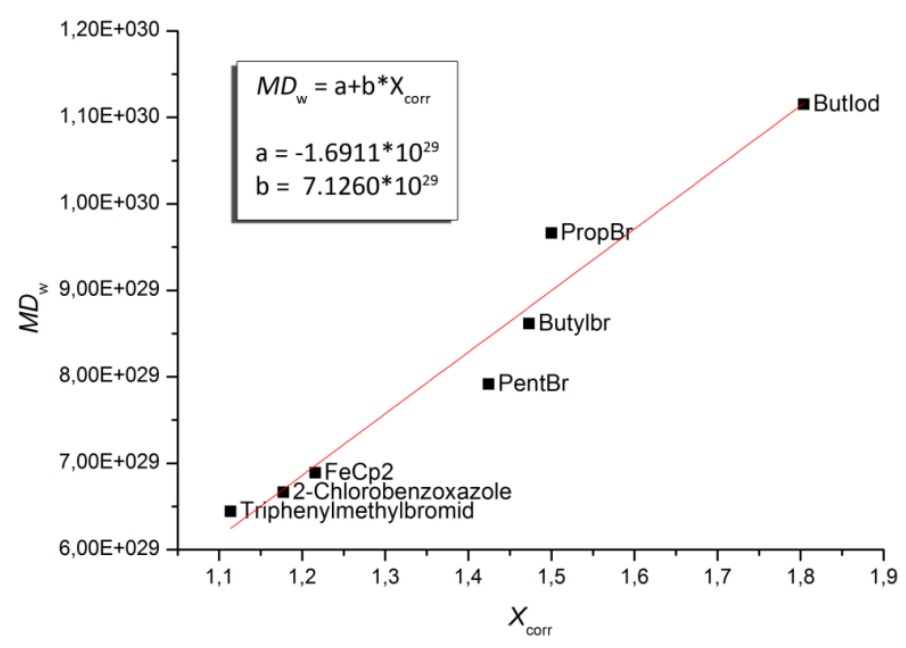

Fig. 2-9. Plot of $M D_{\mathrm{w}}$ vs. $X_{\text {corr }}$ for density correction of molecules with molar van der Waals densities above $5.9 \cdot 10^{29} \mathrm{~g} /\left(\mathrm{mol} \cdot \mathrm{m}^{3}\right)$

With parameter $a$ and $b$ it is possible to apply a density correction on $M W_{\text {det }}$ values for molecules with molar van der Waals densities above $5.9 \cdot 10^{29} \mathrm{~g} /\left(\mathrm{mol} \cdot \mathrm{m}^{3}\right)$ :

$$
M W_{\mathrm{det}, \mathrm{corr}}=M W_{\mathrm{det}} \cdot X_{\mathrm{corr}}
$$

However, equation (27) should be used with caution, since its ability was not proved on a significant amount of model compounds with high molar densities.

Table 2-7. Van der Waals Volumes of selected elements. ${ }^{[11]}$

\begin{tabular}{cc|cc}
\hline Element & $V_{\mathrm{W}}\left[\mathrm{m}^{3}\right]$ & Element & $V_{\mathrm{W}}\left[\mathrm{m}^{3}\right]$ \\
\hline $\mathrm{H}$ & $5.575 \mathrm{E}-30$ & $\mathrm{O}$ & $1.471 \mathrm{E}-29$ \\
$\mathrm{Li}$ & $2.525 \mathrm{E}-29$ & $\mathrm{Si}$ & $3.879 \mathrm{E}-29$ \\
$\mathrm{Na}$ & $4.900 \mathrm{E}-29$ & $\mathrm{P}$ & $2.443 \mathrm{E}-29$ \\
$\mathrm{~K}$ & $8.711 \mathrm{E}-29$ & $\mathrm{~S}$ & $2.443 \mathrm{E}-29$ \\
$\mathrm{Rb}$ & $1.165 \mathrm{E}-28$ & $\mathrm{Cl}$ & $2.245 \mathrm{E}-29$ \\
$\mathrm{Cs}$ & $1.690 \mathrm{E}-28$ & $\mathrm{Br}$ & $2.652 \mathrm{E}-29$
\end{tabular}




\begin{tabular}{cc|cc}
$\mathrm{Mg}$ & $2.169 \mathrm{E}-29$ & $\mathrm{I}$ & $3.252 \mathrm{E}-29$ \\
$\mathrm{C}$ & $2.058 \mathrm{E}-29$ \\
$\mathrm{~N}$ & $1.560 \mathrm{E}-29$ & &
\end{tabular}

\subsubsection{Influence of Deuterated Compounds}

The MW-estimation of the residual solvent peak (THF- $d_{7}, 79 \mathrm{~g} / \mathrm{mol}$ ) gives a MW of $63 \mathrm{~g} / \mathrm{mol}$ that would be underestimated in MW $\left(M W_{\text {err }}=20 \%\right)$. The determined MW fits much better to the protonated THF- $\mathrm{h}_{8}$ species $\left(72 \mathrm{~g} / \mathrm{mol}, M W_{\text {err }}=8 \%\right)$. This is congruent to the nearly similar atomic radius of $\mathrm{D}$ compared to $\mathrm{H}$. According to the abovementioned correlation of the atomic volume and the corresponding diffusion coefficient, it is clear that deuterated molecules diffuse approximately like their protonated counterparts, although they have slightly higher MWs. In the case of TOL- $d_{7}(99 \mathrm{~g} / \mathrm{mol})$ this effect is less pronounced $\left(M W_{\text {det }}=96 \mathrm{~g} / \mathrm{mol}, M W_{\text {err }}=3 \%\right.$ rel. to TOL- $d_{7}$ and $M W_{\text {err }}=-5 \%$ rel. to TOL- $\mathrm{h}_{8}$ ) due to the relative higher MW of toluene. Moreover, especially in the case of multiple THF- $d_{8}$-coordination it is advisable to use the molecular weight of THF- $\mathrm{h}_{8}$ instead of THF- $d_{8}$ to have an accurate MW-determination.

\subsection{DOSY-ECC-MW-Determination of Organometallics and Metal Amides}

On the one hand organometallic compounds tend to aggregate via coordinative bonds that are significantly longer than covalent bonds. Additionally solvent molecules can associate and dissociate in solution. ${ }^{[12]}$ Therefore the space between all atoms is less packed than in

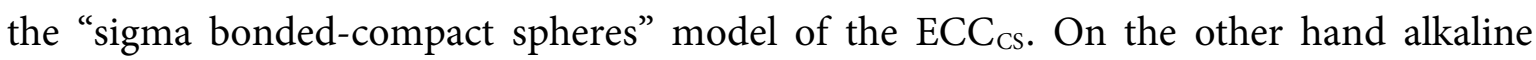
organometallics frequently adopt spherical and ellipsoidal shapes, according to the ringstacking and laddering principle. ${ }^{[1]}$ This is why ECC ${ }_{\text {DSE }}$ for "dissipated spheres and ellipsoids" should be the best calibration curve for the most organometallic compounds. ${ }^{[6]}$

\subsubsection{Structure of LDA in THF Solution}

THF solvated LDA is known to exist exclusively as a disolvated dimer 29 (Fig. 2-10, see also chapter 1.1.1). ${ }^{[14]}$ As a proof of principle a DOSY-ECC-MW-determination of LDA in THF- $d_{8}$ solution $(15 \mathrm{~mm})$ was investigated. In fact, using ECC $\mathrm{DSE}_{\mathrm{D}}$ for the MW- 
determination of LDA $\left(M W_{\text {calc }}=358 \mathrm{~g} / \mathrm{mol}\right)$ in THF- $d_{8}$ gives a MW of $M W_{\text {det }}=347 \mathrm{~g} / \mathrm{mol}$ with a deviation of only $3 \%$ from the calculated MW (Fig. 2-10). ${ }^{[6]}$

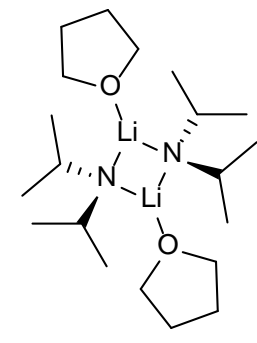

29
DOSY-ECC-MW-determination:

$M W_{\text {calc }}=358 \mathrm{~g} / \mathrm{mol}$

$M W_{\text {det }}=347 \mathrm{~g} / \mathrm{mol}$

$M W_{\text {err }}=3 \%$

Fig. 2-10. DOSY-ECC-MW-determination of LDA $(15 \mathrm{~mm})$ in THF- $d_{8}$ solution by applying ECC $\mathrm{DSE}_{\mathrm{DSE}}$ PhN $(15 \mathrm{~mm})$ was used as internal reference with $D_{\text {ref,fix }}=-8.8812$.

\subsubsection{Structure of LDA in Toluene Solution ${ }^{11}$}

LDA is a very prominent reagent that plays a key role in organic synthesis, serving as a base par excellence for a broad range of deprotonation reactions (see e.g. Scheme 1-2). However, the state of aggregation in solution in the absence of donor bases was unclear. Kim and Collum et al. analyzed [ $\left[{ }^{6} \mathrm{Li}\right] \mathrm{LDA}$ and $\left[{ }^{6} \mathrm{Li},{ }^{15} \mathrm{~N}\right] \mathrm{LDA}$ in hexane by ${ }^{6} \mathrm{Li}$ and ${ }^{15} \mathrm{~N} \mathrm{NMR}$ spectroscopy and observed a mixture of as many as five cyclic oligomers. ${ }^{[12 a]}$ They anticipated that the main cyclic aggregates corresponded to dimers, trimers and higher oligomers. Unfortunately a quantification of these observations was not possible because " $a$ severe overlap renders the effort required for a detailed study unjustifiable". This is why these oligomers have not been characterized further.

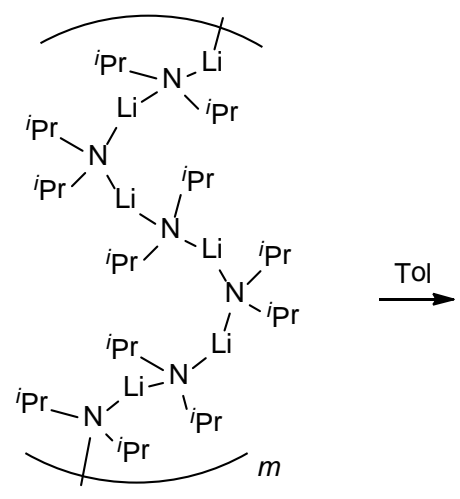

55

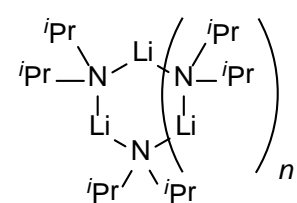

56a: $n=1$

56b: $n=2$

56c: $n=3$

56d: $n=4$

Scheme 2-1. LDA in the solid state and in toluene solution.

\footnotetext{
${ }^{11}$ A revised version of my publication: R. Neufeld, M. John, D. Stalke, Angew. Chem., 2015, 127, 7100-7104;
} Angew. Chem. Int. Ed., 2015, 54, 6994-6998. 
LDA is polymeric in the solid state and shows little solubility in toluene (Scheme 2-1). The highest concentrations that could be observed at RT were in the range of 7-15 mm. In the ${ }^{7} \mathrm{Li}$ spectrum only one broad signal at $2.81 \mathrm{ppm}$ is present that broadens up with decreasing temperature. This circumstance makes it impossible to distinguish between any oligomers from the ${ }^{7} \mathrm{Li}-\mathrm{NMR}$ experiment (Fig. 2-11a). However, at RT in the ${ }^{1} \mathrm{H}$ spectrum two sets of two main signals corresponding to the $\mathrm{a}-\mathrm{CH}(3.12 \mathrm{ppm}$ and $3.01 \mathrm{ppm})$ and $\mathrm{CH}_{3}$ group (1.14 ppm and $1.11 \mathrm{ppm}$ ) are present (species $\mathbf{A}$ and $\mathbf{B}$ Fig. 2-11b). A third compound with very low intensity was also evidenced by an additional $\alpha-\mathrm{CH}$ signal at $3.19 \mathrm{ppm}$ (species C). Due to its poor intensity it was not possible to determine the diffusion coefficient of this third compound at RT. Although, the other two main signals at $3.12 \mathrm{ppm}$ and $3.01 \mathrm{ppm}$ show some overlap, their self-diffusion was measureable via DOSY-NMR.

a)

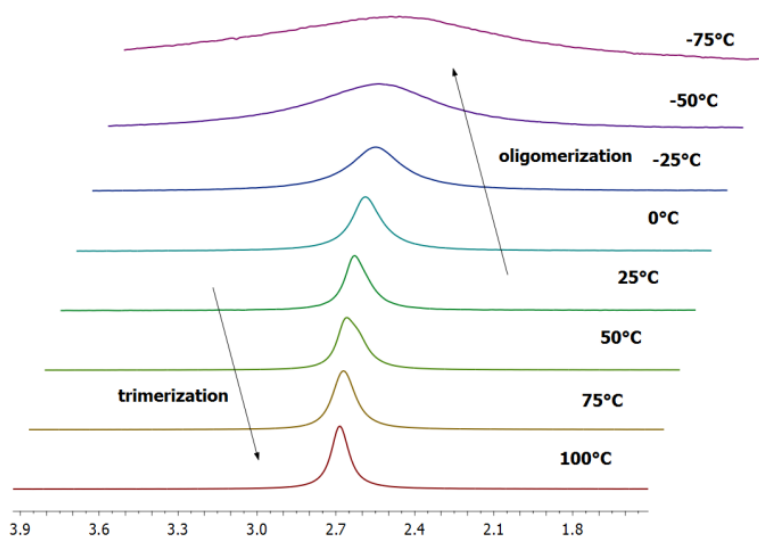

b)

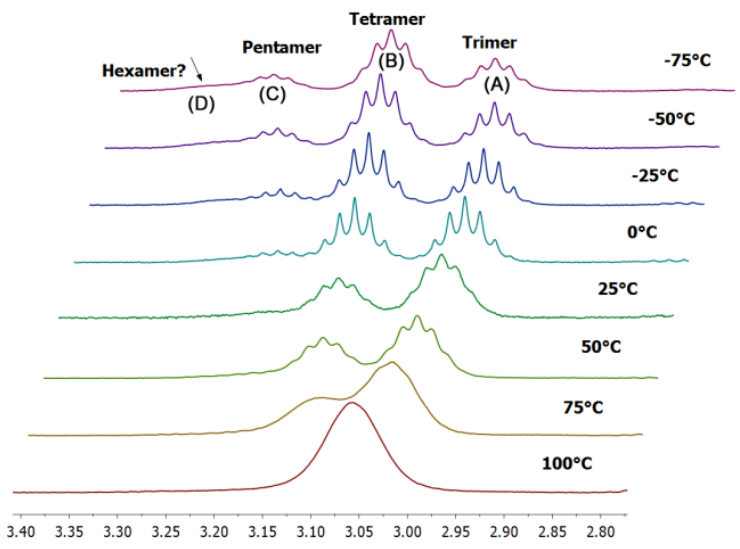

Fig. 2-11. a) ${ }^{7} \mathrm{Li}$ - and b) ${ }^{1} \mathrm{H}$-superposition plot of LDA and its $a-\mathrm{CH}$ signals in TOL- $d_{8}$ at different temperatures.

From DOSY-ECC-MW-determination (Table 2-8B) species A agrees best with a trimer 56a $\left(M W_{\text {calc }}=321 \mathrm{~g} / \mathrm{mol}, M W_{\text {det }}=318 \mathrm{~g} / \mathrm{mol}, M W_{\text {err }}=1 \%\right.$, Scheme 2-1 $)$ and species B with tetramer $56 \mathbf{b}\left(M W_{\text {calc }}=428 \mathrm{~g} / \mathrm{mol}, M W_{\text {det }}=390 \mathrm{~g} / \mathrm{mol}, M W_{\text {err }}=9 \%\right)$. Careful integration of both signals at $25^{\circ} \mathrm{C}$ reveals that 56a and $\mathbf{5 6 b}$ exist together in a ratio of 2:1 (A-Table 12 in the appendix). It is also evident that dimers, like those proposed by Kim et al. are not present in the mixture $\left(M W_{\text {err }}=-48 \%\right.$ and $\left.-143 \%\right)$ at any temperature. The other low field shifted species $\mathbf{C}$ with weak intensity has to be a bigger aggregate than the tetramer. At $-50^{\circ} \mathrm{C}$ the integral of $\mathbf{C}$ increases significantly at the expense of species $\mathbf{A}$. The signal separation was suitable for the DOSY-ECC-MW-determination (A-Figure 3 in the 
appendix). Table 2-8a illustrates that $\mathbf{C}$ shows the best agreement with a pentameric LDAaggregate 56c $\left(M W_{\text {calc }}=536 \mathrm{~g} / \mathrm{mol}, M W_{\text {det }}=520 \mathrm{~g} / \mathrm{mol}, M W_{\text {err }}=3 \%\right)$.

Table 2-8. DOSY-ECC-MW-determination of LDA in TOL- $d_{8}$ at various temperatures. ${ }^{\text {a) }}$

\begin{tabular}{lcccccc}
\hline & & Dimer & Trimer & Tetramer & Pentamer & Hexamer \\
& & $(214 \mathrm{~g} / \mathrm{mol})$ & $(321 \mathrm{~g} / \mathrm{mol})$ & $(428 \mathrm{~g} / \mathrm{mol})$ & $(536 \mathrm{~g} / \mathrm{mol})$ & $(643 \mathrm{~g} / \mathrm{mol})$ \\
& $M W_{\text {det }}$ & $M W_{\text {err }}$ & $M W_{\text {err }}$ & $M W_{\text {err }}$ & $M W_{\text {err }}$ & $M W_{\text {err }}$ \\
{$[\mathrm{g} / \mathrm{mol}]$} & {$[\%]$} & {$[\%]$} & {$[\%]$} & {$[\%]$} & {$[\%]$} \\
\hline a) $-50^{\circ} \mathrm{C}$ & & & & & & \\
Species A & 332 & -55 & $-\mathbf{3}$ & 22 & 38 & 48 \\
Species B & 423 & -98 & -32 & $\mathbf{1}$ & 21 & 34 \\
Species C & 520 & -143 & -62 & -21 & $\mathbf{3}$ & 19 \\
\hline b) $+25^{\circ} \mathrm{C}$ & & & & & & \\
Species A & 318 & -48 & $\mathbf{1}$ & 26 & 41 & 51 \\
Species B & 390 & -82 & -21 & $\mathbf{9}$ & 27 & 39 \\
\hline c) $+100^{\circ} \mathrm{C}$ & & & & & & \\
Species A & 333 & -56 & -4 & 22 & 38 & 48
\end{tabular}

a) $\mathrm{ECC}_{\mathrm{DSE}}^{\mathrm{TOL}}$ was used to determine the MWs. The accuracy of this method is in the range of $M W_{\text {err }} \leq \pm 9 \%$. None of the species show accordance with the dimer $\left(M W_{\text {err }} \geq-48 \%\right)$.

The MW estimation of the residual diisopropyl amine present in solution $(\mathrm{DA}(\mathrm{H})$, $M W_{\text {calc }}=101 \mathrm{~g} / \mathrm{mol}, M W_{\text {det }}=100 \mathrm{~g} / \mathrm{mol}, M W_{\text {err }}=1 \%$, A-Table 11 in the appendix) was also possible. The good agreement with the calculated MW gives evidence that DA(H) does not coordinate to any oligomeric species. This result is consistent with previous investigations, which showed that $\mathrm{DA}(\mathrm{H})$ is a very poor ligand for $\mathrm{LDA} \cdot{ }^{[14 a]}$ At $-50^{\circ} \mathrm{C}$ an additional multiplet appears that belongs to oligomer $\mathbf{D}$ at the left hand side of the signal attributed to the pentamer $\mathbf{C}$ (Fig. 2-11a). Unfortunately this signal was too weak for a MW-determination. Further cooling did not improve the signal-to-noise ratio due to a reduced solubility of LDA in toluene at temperatures below $-50^{\circ} \mathrm{C}$. In 1999 , Rutherford and Collum showed by low temperature ${ }^{6} \mathrm{Li}$ and ${ }^{15} \mathrm{~N}$ NMR spectroscopy that the lighter congener of LDA, lithium diethyl amide (LiDEA) can exist as several oligomers in THF and oxetane solutions (Fig. 2-12). ${ }^{[13]}$ In neat THF or oxetane LiDEA is a cyclic dimer 57. At lower donor base concentrations cyclic oligomers appear. At low THF concentrations (2-10 equiv.) a cyclic trimer $\mathbf{5 8}$ and a four-rung-ladder $\mathbf{5 9}$ are formed. Higher order ladders were not observed within the solubility limits of LiDEA, but at sub-stoichiometric oxetane concentrations they noticed a relatively complex LiDEA equilibrium of cyclic dimers, trimers and ladders of tetramers, pentamers and hexamers (57-61). While LiDEA tend to 
form ladder sructures, an increased bulk of the R groups favors cyclic arrangements. E.g. the donor base-free lithium hexamethyldisilazide adopts a cyclic trimeric structure $\mathbf{4 0}$ in the solid state $\mathrm{e}^{[39 b]}$ and exists as a cyclic tetramer- dimer mixture in hydrocarbons (see chapter 1.1.2) ${ }^{[51]}$

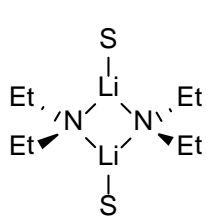

57

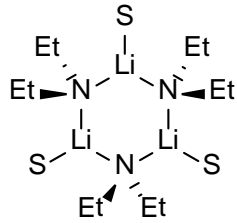

58

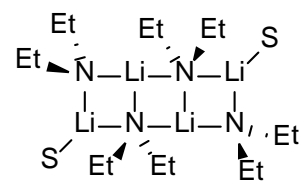

59

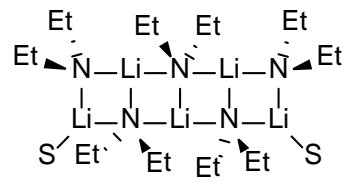

60

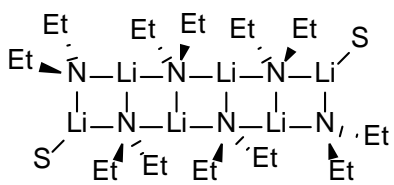

61

Fig. 2-12. Aggregation of lithium diethyl amide (LiDEA) in neat oxetane or pentane/toluene mixtures as cosolvent. ${ }^{[113]}$

Similarly lithium tetramethylpiperidide adopts a cyclic trimer $\mathbf{3 1}$ and tetramer $\mathbf{3 2}$ in the solid state ${ }^{[33-34]}$ and appears to form both cyclic oligomers in pentane (see chapter 1.1.1). ${ }^{[34 b]}$ In view of this trend and the bulkiness of the ${ }^{i} \mathrm{Pr}$-groups, the assumption that the signal from oligomer D stems from the cyclic LDA-hexamer 56d appears to be valid. Cooling the sample shifts the position of the oligomer-equilibrium. While the tetramer concentration increases, that of the trimer decreases. Obviously low temperatures stabilize the higher aggregates, due to entropy. The conversion of the trimer to the corresponding oligomers is also reflected in the ${ }^{7} \mathrm{Li}$ NMR spectrum (Fig. 2-11a). The ${ }^{7} \mathrm{Li}$ signal becomes broader at lower temperature. This could be due to a relatively faster quadrupolar relaxation or additionally due to the increase of oligomeric structures. Warming up the solution causes the opposite trend. The oligomer concentration decreases, while the trimer concentration increases. At $+50^{\circ} \mathrm{C}$ a shoulder at the main ${ }^{7} \mathrm{Li}$ signal is apparent, revealing two main species: the trimer 56a and the tetramer 56b. In the ${ }^{1} \mathrm{H}$ NMR spectrum at $+100^{\circ} \mathrm{C}$ all aggregates coalesce to one set of signals at $3.06 \mathrm{ppm}$ and $1.10 \mathrm{ppm}$, respectively. The ECC$\mathrm{MW}$-determination estimates a $\mathrm{MW}$ of $M W_{\mathrm{det}}=333 \mathrm{~g} / \mathrm{mol}$ that fits best to the trimeric LDA species 56a with an MW-deviation of only $\mathrm{MW}_{\text {err }}=-4 \%($ Table $2-8 \mathrm{c}){ }^{\left[{ }^{[14]}\right.}$ 


\subsubsection{Structure of $\mathrm{Na}$-Indenide in THF Solution ${ }^{12}$}

Alkali metal indenides are important precursors for the synthesis of metallocenes of the main group and transition metals. Without donating ligands like ethers or chelating crown ethers they build up polymeric stack structures. ${ }^{[115]}$ The solid-state structure of donor basefree Na-indenide is unknown. With donating ligands like PMDETA or crone ethers Li- and Na-indenide form contact ion pairs (CIP). ${ }^{[16]}$ With ammonia solvent separated ion pairs (SSIP) are formed. ${ }^{[7]}$ However, the aggregation of Na-indenide in solution is still unclear. One reason for that may be the relative bad NMR properties of the sodium nucleus that has a spin of 3/2. This quadrupole results in broad lines that get even broader with asymmetry in the environment of the sodium nucleus. ${ }^{1} \mathrm{H}$-DOSY experiments are independent of that nucleus. Therefore, $\mathrm{Na}$-indenide is an interesting candidate for discovering its aggregation in THF solution by employing the new DOSY-ECC-MW-determination methodology. The most feasible species are the THF solvated monomers (M1-M4) and the dimers D1-D2 bearing $n$ THF molecules (Fig. 2-13).

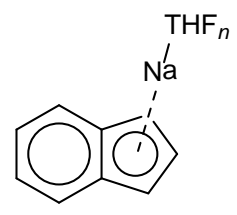

62

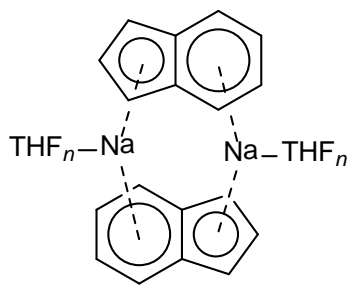

63

M1: $n=1$

M2: $n=2$

M3: $n=3$

M4: $n=4$

D1: $n=1$

D2: $n=2$

Fig. 2-13. Most plausible Na-indenide species in THF solution.

The molar density for all species is between $M D_{\mathrm{w}}=5.07$ and $5.43 \cdot 10^{29} \mathrm{~g} /\left(\mathrm{mol} \cdot \mathrm{m}^{3}\right)$ which ensures that those aggregates are suitable for all developed ECCs. At room temperature (RT) the DOSY-ECC-MW-determination estimates a $M W$ of $M W_{\text {det }}=331 \mathrm{~g} / \mathrm{mol}$. The comparison of the estimated MW with the most likely Na-indenide species is shown in Table 2-9. Both dimers with two- $\left(\right.$ D1: $\left.M W_{\text {err }}=21 \%\right)$ and four THF molecules (D2: $\left.M W_{\text {err }}=41 \%\right)$ can be excluded. The same is true for the mono- $\left(\right.$ M1: $\left.M W_{\text {err }}=-57 \%\right)$ and

\footnotetext{
${ }^{12}$ Includes revised parts of my publication: R. Neufeld, D. Stalke, Chem. Sci. 2015, 6, 3354-3364.
} 
Table 2-9. DOSY-ECC-MW-determination of Na-indenide ( $15 \mathrm{mM})$ in THF- $d_{8}$ at various temperatures. TMB (15 mM) was used as internal reference and $\mathrm{ECC}_{\mathrm{DSE}}$ to determine the MWs. ${ }^{\text {a) }}$

\begin{tabular}{cccccc}
\hline Species & $\mathrm{n}$ & $\begin{array}{c}M W_{\text {calc }} \\
{[\mathrm{g} / \mathrm{mol}]}\end{array}$ & \multicolumn{3}{c}{$\begin{array}{c}M W_{\text {err }} \\
{[\%]}\end{array}$} \\
\hline M1 & 1 & 210 & -84 & -57 & -36 \\
M2 & 2 & 282 & -37 & -17 & -1 \\
M3 & 3 & 354 & -9 & 7 & 19 \\
M4 & 4 & 426 & 10 & 22 & 33 \\
D1 & 1 & 420 & $8^{\text {b) }}$ & 21 & 32 \\
D2 & 2 & 564 & 32 & 41 & 49 \\
\hline Indene & & 111 & 4 & 2 & -30 \\
HMDS(H) & & 161 & -5 & -5 & -1
\end{tabular}

a) DOSY-ECC-MW-determination of Na-Indenide in THF- $d_{8}$ solution gave following results: $M W_{\text {det }}\left(-50^{\circ} \mathrm{C}\right)=386 \mathrm{~g} / \mathrm{mol}, M W_{\text {det }}\left(25^{\circ} \mathrm{C}\right)=331 \mathrm{~g} / \mathrm{mol}, M W_{\text {det }}\left(+60^{\circ} \mathrm{C}\right)=286 \mathrm{~g} / \mathrm{mol}$.

b) The disolvated dimer D1 $\left(M W_{\text {err }}=8 \%\right)$ would also fit to the estimated $\mathrm{MW}$, but this aggregation makes in this context chemically not much sense.

disolvated monomers (M2: $\left.M W_{\text {err }}=-17 \%\right)$ that can also be easily excluded. The trisolvated monomer $\left(\right.$ M3: $\left.M W_{\text {err }}=7 \%\right)$ gives the best match. Such a three-fold THF coordination fits perfectly many crystal structures of THF solvated sodium cyclopentadienide derivatives. ${ }^{[117]}$ Without difficulty it is also possible to identify the signals of remaining indene $\left(M W_{\mathrm{err}}=2 \%\right)$ and hexamethyldisilazane $\left(\operatorname{HMDS}(\mathrm{H}), M W_{\mathrm{err}}=-5 \%\right)$. Those very accurate MWs indicate that the exchange of the latter with Na-indenide M3 is very slow or not present. Otherwise the estimated MWs of indene or HMDS(H) should be much higher. At $-50^{\circ} \mathrm{C}$ it is obvious that the equilibrium of $\mathrm{Na}$-indenide changes to a higher $\mathrm{MW}$ of $M W_{\mathrm{det}}=386 \mathrm{~g} / \mathrm{mol}$. That $\mathrm{MW}$ is right in between three- $\left(\mathbf{M} 3: M W_{\mathrm{err}}=-9 \%\right)$ and four-fold (M4: $M W_{\text {err }}=10 \%$ ) THF-coordinated Na-indenide, indicating that a fourth THF coordination is attractive at low temperatures. Again, indene $\left(M W_{\text {err }}=4 \%\right)$ and $\operatorname{HMDS}(\mathrm{H})\left(M W_{\text {err }}=-5 \%\right)$ are not involved in that Na-indenide-THF equilibrium. By warming up the THF solution to $+60^{\circ} \mathrm{C}$ the opposite trend is evident. The ECC-MWdetermination estimates for Na-indenide a much lower $M W$ of $M W_{\text {det }}=286 \mathrm{~g} / \mathrm{mol}$ that would fit to a disolvated Na-indenide monomer $\left(\mathbf{M} 2: M W_{\text {err }}=-1 \%\right)$ but additionally the MW of indene rises significantly to $\left(M W_{\text {det }}=158 \mathrm{~g} / \mathrm{mol}, M W_{\text {err }}=-37 \%\right)$. This behavior indicates a rapid exchange of $\mathrm{Na}$-indenide and indene at high temperatures producing a merged MW for both. Anyway, $\operatorname{HMDS}(\mathrm{H})$ is still not involved in that equilibrium $\left(M W_{\text {err }}=-1 \%\right)$ most likely due to its higher basicity and steric demand, compared with indene $\left(\mathrm{p} K_{\mathrm{s}}=26\right.$ vs 20$){ }^{\left[{ }^{[118]}\right.}$ 


\subsubsection{Structure of $[t-B u L i]_{4} \cdot 4\left[\mathrm{Me}_{2} \mathrm{NC}_{6} \mathrm{H}_{4} \mathrm{Li}\right]_{4}$ in Toluene Solution ${ }^{13}$}

In 2012 A.-C. Pöppler et al. showed that ortho lithium dimethylanilide $\left(\mathrm{Me}_{2} \mathrm{NC}_{6} \mathrm{H}_{4} \mathrm{Li}\right)$ crystallizes in the presence of ${ }^{t} \mathrm{BuLi}$ as a separated lithium organic aggregate $[t-\mathrm{BuLi}]_{4} \cdot 4\left[\mathrm{Me}_{2} \mathrm{NC}_{6} \mathrm{H}_{4} \mathrm{Li}\right]_{4} 64$ in the same crystal (Fig. 2-14). ${ }^{[119]}$ Dissolving crystals of 64 in TOL- $d_{8}$ resulted in an unexpectedly complicated ${ }^{7} \mathrm{Li}-\mathrm{NMR}$ spectrum that shows five relatively sharp distinguishable signals over a range of nearly $2.5 \mathrm{ppm}$. In the ${ }^{7} \mathrm{Li}$-DOSY experiment these species display a monotonically decreasing diffusion coefficient (Fig. 2$15)$.
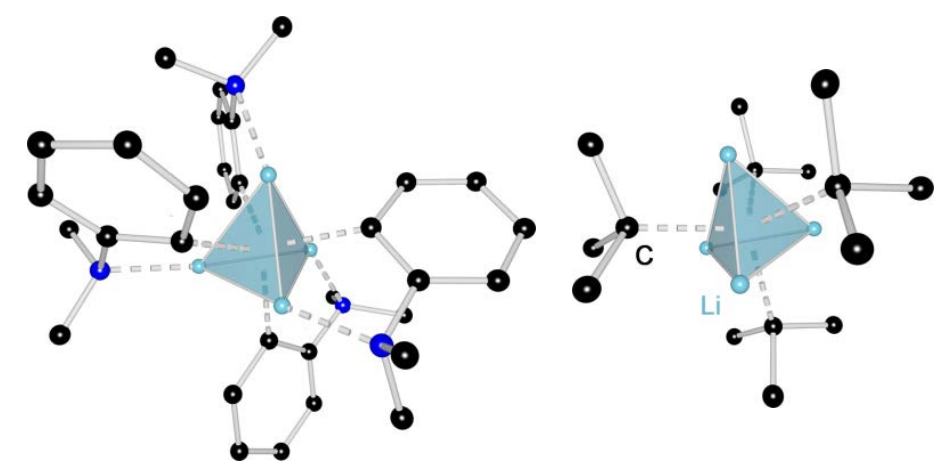

64

Fig. 2-14. Crystal structure of $[t-\mathrm{BuLi}]_{4} \cdot 4\left[\mathrm{Me}_{2} \mathrm{NC}_{6} \mathrm{H}_{4} \mathrm{Li}\right]_{4} 64$ with hydrogen atoms omitted for clarity.

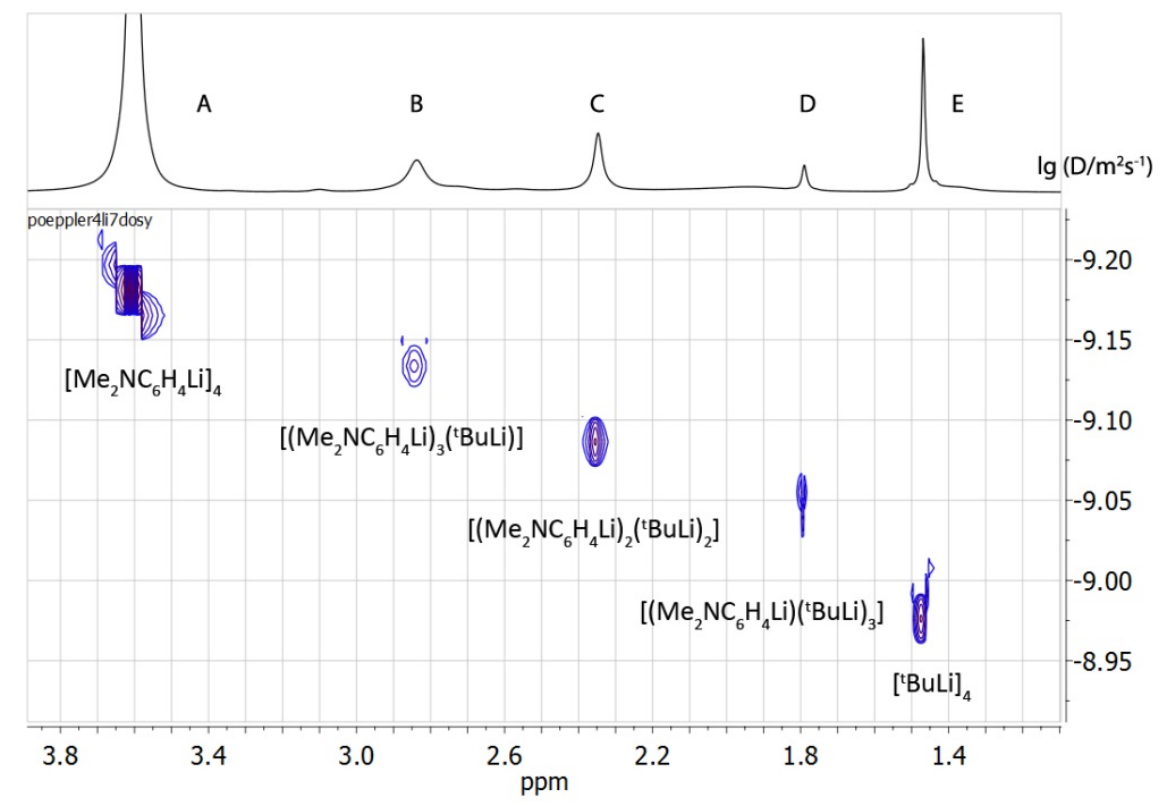

Fig. 2-15. ${ }^{7} \mathrm{Li}$-DOSY spectrum of $[t \text {-BuLi }]_{4} \cdot 4\left[\mathrm{Me}_{2} \mathrm{NC}_{6} \mathrm{H}_{4} \mathrm{Li}\right]_{4} 64$ in TOL- $d_{8}$ solution.

\footnotetext{
${ }^{13}$ Includes revised parts of my publication: R. Neufeld, D. Stalke, Chem. Sci. 2015, 6, 3354-3364.
} 
It was anticipated that each species (A-E) represents a tetramer with a consecutive substitution of one ortho lithium anilide by one ${ }^{t} \mathrm{BuLi}$ moiety. However, no verifications of these results using quantitative MW determinations were performed. Reinvesting in that issue and using the diffusion measurements of A.-C. Pöppler et al. it was possible to determine the MWs of all species a posteriori by applying the DOSY-ECC-MWdetermination. Taking the residual proton signal of the solvent (TOL- $\left.d_{7}\right)$ as internal reference gives very good results with the proposed structures A-E with an error smaller $\pm 5 \%$ (Table 2-10).

Table 2-10. DOSY-ECC-MW-determination of crystalline $[t-\mathrm{BuLi}]_{4} \cdot 4\left[\mathrm{Me}_{2} \mathrm{NC}_{6} \mathrm{H}_{4} \mathrm{Li}\right]_{4} 64$ in TOL- $d_{8}$ solution. $E C C_{D S E}$ and TOL- $d_{7}$ as internal reference with $\log D_{\text {ref, fix }}=-8.7289$ were used to determine the MWs.

\begin{tabular}{ccccc}
\hline & Species & $\begin{array}{c}M W_{\text {calc }} \\
{[\mathrm{g} / \mathrm{mol}]}\end{array}$ & $\begin{array}{c}M W_{\text {det }} \\
{[\mathrm{g} / \mathrm{mol}]}\end{array}$ & $\begin{array}{c}M W_{\text {err }} \\
{[\%]}\end{array}$ \\
\hline A & {$\left[\mathrm{Me}_{2} \mathrm{NC}_{6} \mathrm{H}_{4} \mathrm{Li}\right]_{4}$} & 508 & 527 & -4 \\
B & {$\left[\left(\mathrm{Me}_{2} \mathrm{NC}_{6} \mathrm{H}_{4} \mathrm{Li}\right)_{3}\left({ }^{t} \mathrm{BuLi}\right)\right]$} & 445 & 435 & 2 \\
$\mathrm{C}$ & {$\left[\left(\mathrm{Me}_{2} \mathrm{NC}_{6} \mathrm{H}_{4} \mathrm{Li}\right)_{2}\left({ }^{(} \mathrm{BuLi}\right)_{2}\right]$} & 382 & 367 & 4 \\
$\mathrm{D}$ & {$\left[\left(\mathrm{Me}_{2} \mathrm{NC}_{6} \mathrm{H}_{4} \mathrm{Li}\right)\left({ }^{t} \mathrm{BuLi}\right)_{3}\right]$} & 319 & 316 & 1 \\
E & {$\left[{ }^{\mathrm{BuLi}}\right]_{4}$} & 256 & 244 & 5
\end{tabular}

\subsubsection{Structure of MHMDS with Ammonia as Donorbase in Toluene Solution}

Previous investigations on MHMDS with ammonia as donor base revealed unprecedented aggregation motifs in the solid state (Fig. 2-16): ${ }^{[1]}$
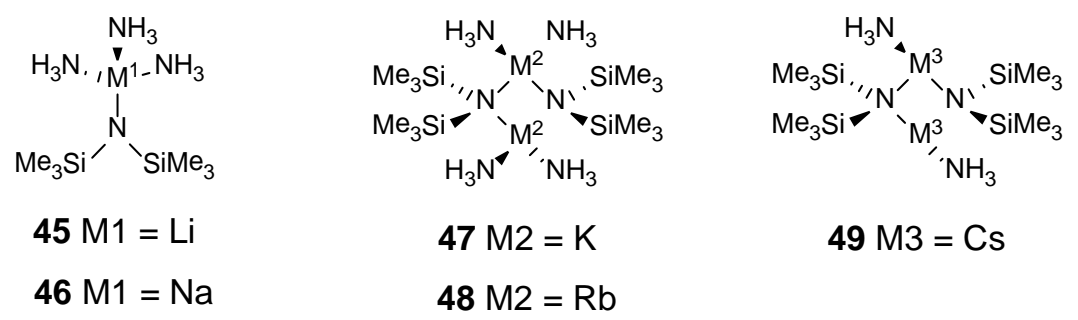

Fig. 2-16. Aggregation motifs of MHMDS ( $\mathrm{M}=\mathrm{Li}, \mathrm{Na}, \mathrm{K}, \mathrm{R}$ band $\mathrm{Cs})$ with ammonia as donor base in the solid state. ${ }^{[1]}$

Li- and NaHMDS form trisolvated monomers 45 and 46, stabilized by intermolecular hydrogen bonds (HB) enabling the possibility of HB-stabilized transition states in synthesis. Ammoniacates of KHMDS and RbHMDS form tetrasolvated dimers $\mathbf{4 7}$ and $\mathbf{4 8}$ 
with two ammonia molecules which coordinate each metal cation. In the case of CsHMDS the solvation number of ammonia is decreased leading to the disolvated CsHMDS dimer 49, where open coordination sides are stabilized by several s-block agnostic-like $\mathrm{Si}-\mathrm{CH}_{3} \cdots \mathrm{Cs}$ interactions. ${ }^{[1]}$ All above mentioned crystal structures were derived from concentrated ammonia solutions where an excess of liquid ammonia was present. However, addition of toluene to the mother liquor and warming up the solution to $25^{\circ} \mathrm{C}$ and subsequent crystallization at $-45^{\circ} \mathrm{C}$ resulted in the formation of further crystal structures which will be presented in the following section.

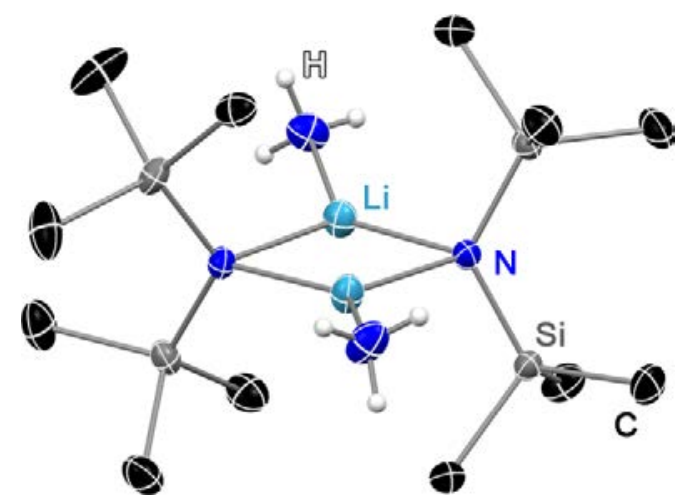

65

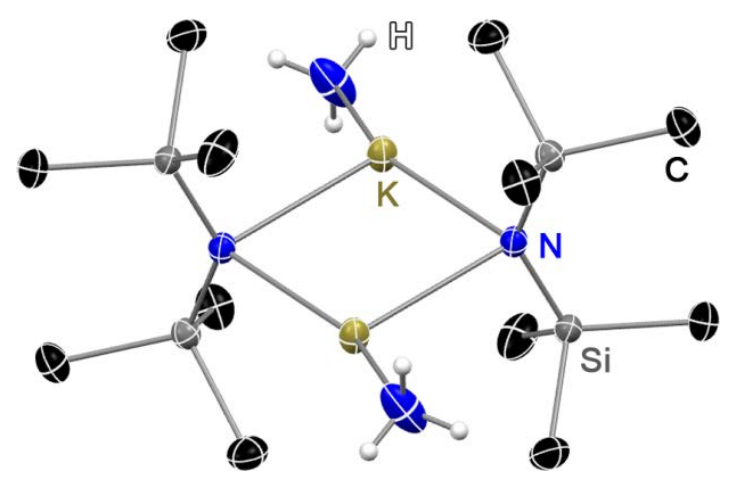

67

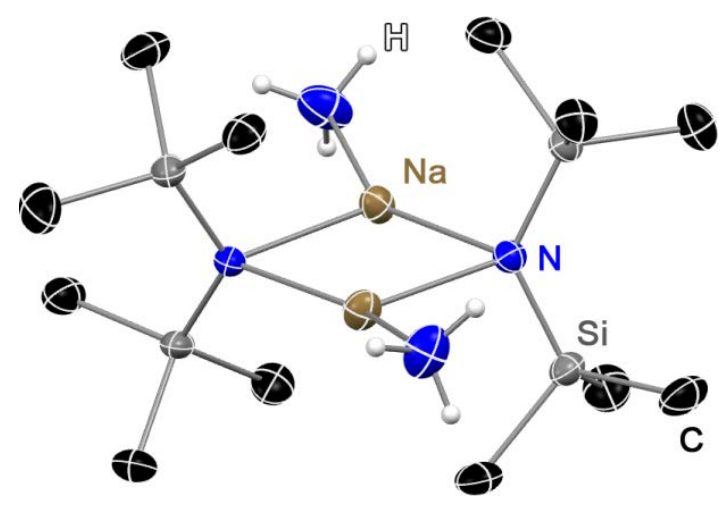

66

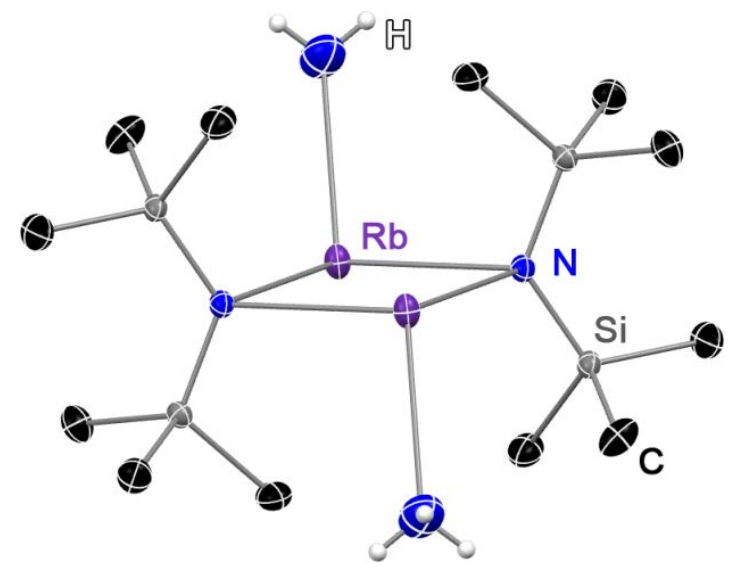

68

Fig. 2-17. Dimeric structure of MHMDS $(\mathrm{M}=\mathrm{Li}, \mathrm{Na}, \mathrm{K}$ and $\mathrm{Rb})$ with one equivalent of $\mathrm{NH}_{3}$ in the solid state. Anisotropic displacement parameters are depicted at the 50\% probability level. Carbon attached hydrogen atoms are omitted for clarity. Selected bond lengths, angles and crystallographic details are displayed in chapters 4.4 .1 to 4.4 .4 . 


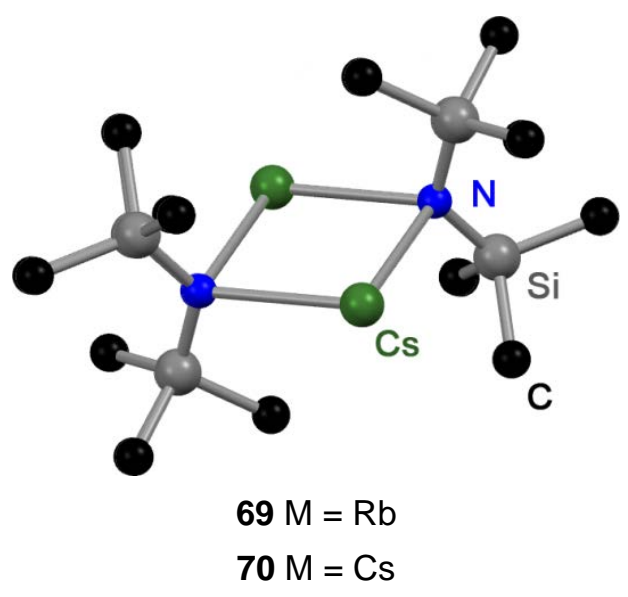

Fig. 2-18. Dimeric structure of donor-base-free $\mathrm{Rb}$ - and CsHMDS in the solid state. ${ }^{[43]}$ Carbon bound hydrogen atoms are omitted for clarity.

a)

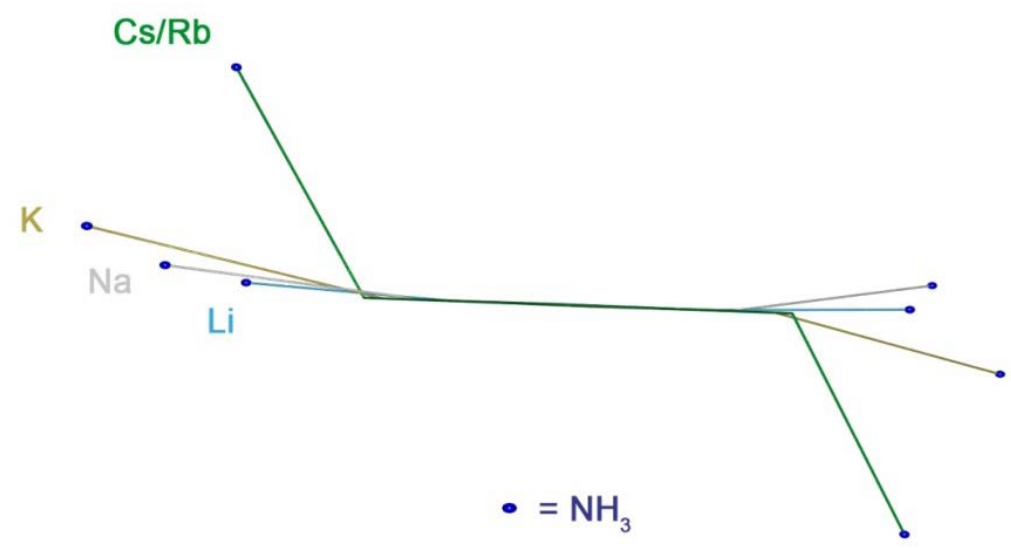

b)

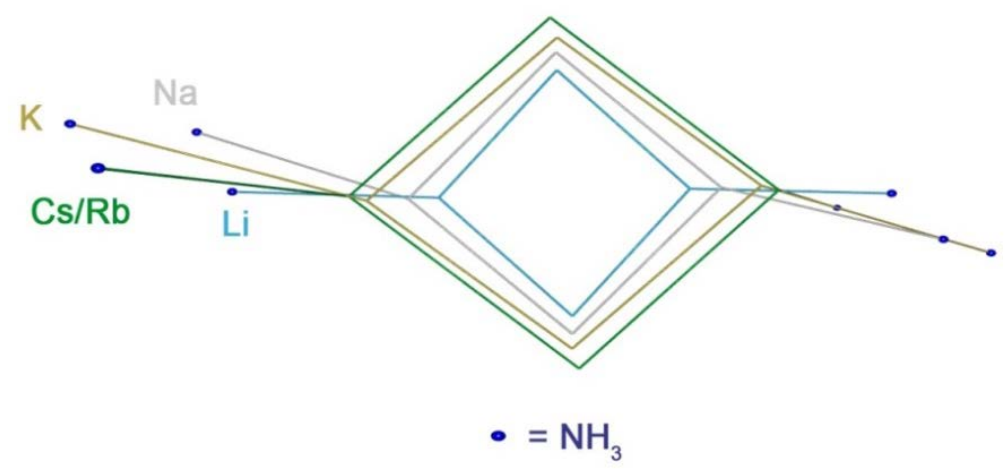

Fig. 2-19. Superposition plot of all disolvated $\left[\left(\mathrm{NH}_{3}\right) \mathrm{MHMDS}\right]_{2}(\mathrm{M}=\mathrm{Li}, \mathrm{Na}, \mathrm{K}, \mathrm{Rb}$ and $\mathrm{Cs})$ ammoniacates. a) View along the planar $\left[\mathrm{N}_{2} \mathrm{M}_{2}\right]$-ring. b) View orthogonal to the planar $\left[\mathrm{N}_{2} \mathrm{M}_{2}\right]$-ring. With increasing metal size the coordination angle of $\mathrm{NH}_{3}$ increases, too, due to inter- and intramolecular Si-CH $\mathrm{CH}_{3} \cdots \mathrm{M}$ interactions which occupy the coordination sphere of the alkali metal. 
Due to the increased reaction temperature $\left(25^{\circ} \mathrm{C}\right.$ instead of $\left.-33^{\circ} \mathrm{C}\right)$ excess ammonia was allowed to evaporate. Stochiometric amounts of ammonia and MHMDS ( $\mathrm{M}=\mathrm{Li}, \mathrm{Na}, \mathrm{K}$ and $\mathrm{Rb}$ ) yielded disolvated dimers 65-69 (Fig. 2-17) with an aggregation mode similar to that of $\left[\left(\mathrm{H}_{3} \mathrm{~N}\right) \mathrm{CsN}\left(\mathrm{SiMe}_{3}\right)_{2}\right]_{2} 49$ in Fig. 2-16. In the case of CsHMDS a donor-base-free dimer 70 without any coordination of ammonia was identified (Fig. 2-18). This structure was already characterized by Neander and Behrens in 1999. ${ }^{[43]}$ Interestingly, at least in the solid state at low ammonia concentrations the Lewis soft cesium cation prefers Lewis soft $\mathrm{Si}-\mathrm{CH}_{3}$..Cs interactions over the coordination of Lewis hard ammonia molecules. Since there are no conspicuous bond lengths in respect to the already characterized MHMDS ammoniacates $^{[1]}$ a detailed bond length discussion was redundant. However, selected bond lengths, angles and crystallographic details are given in chapters 4.4.1 to 4.4.4. In respect to the planar $\left[\mathrm{M}_{2} \mathrm{~N}_{2}\right]$-ring the coordination angle of the ammonia molecules grows with increasing metal size (Fig. 2-19). This is due to inter- and intramolecular $\mathrm{Si}-\mathrm{CH}_{3} \cdots \mathrm{M}$ interactions which saturate the coordination sphere of the metal cation. The same was already observed in $\left[\left(\mathrm{H}_{3} \mathrm{~N}\right) \mathrm{CsN}\left(\mathrm{SiMe}_{3}\right)_{2}\right]_{2}$ 49. ${ }^{[1]}$

A unique NaHMDS intermediate $\mathbf{7 1}$ was crystallized from a solution which was warmed up to $0^{\circ} \mathrm{C}$ instead of $+25^{\circ} \mathrm{C}$ (Fig. 2-20). Compound 71 represents an open dimer that is stabilized by an intramolecular HB of a second NaHMDS molecule.

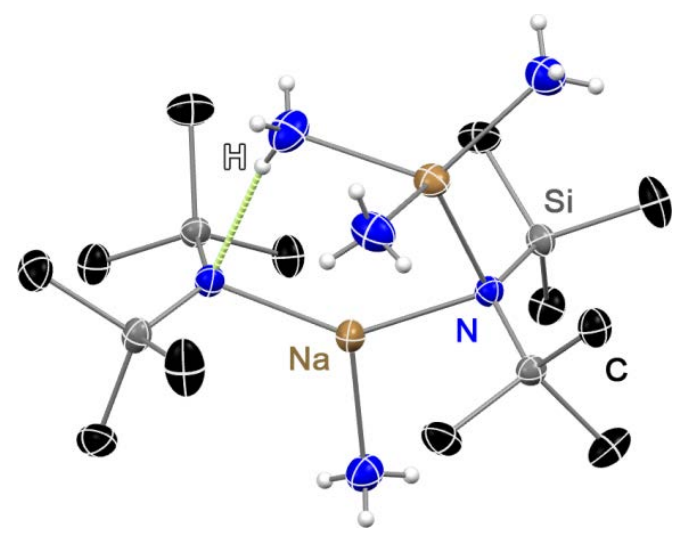

71

Fig. 2-20. Structure of NaHMDS intermediate $\mathbf{7 1}$ in the solid state. Anisotropic displacement parameters are depicted at the $50 \%$ probability level. Carbon bound hydrogen atoms are omitted for clarity. Selected bond lengths and angles and crystallographic details are displayed in chapter 4.4.5.

From MHMDS ammoniacates 65-71 an aggregation-deaggregation mechanism can be proposed that is depicted in Scheme 2-2. Successive addition of ammonia to dimer $\mathbf{A}$ would produce the monosolvated ${ }^{[120]}$ compound $\mathbf{B}$, disolvated $\mathbf{C}$ and trisolvated $\mathbf{D}$. The latter structure was observed in NaHMDS.3THF and also in mixed alkalimetal 
HMDS.3THF aggregates (e.g. M1 $=\mathrm{Li}$ and $\mathrm{M} 2=\mathrm{Na}, \mathrm{K}) .{ }^{[53]} \mathrm{A}$ fourth addition of $\mathrm{NH}_{3}$ would result in the tetrasolvated structure $\mathbf{E}$ that was identified in compound $\mathbf{4 7}$ and $\mathbf{4 8}$. Migration of one $\mathrm{NH}_{3}$ molecule to the second metal, followed by a M-N cleavage leads to structure $\mathbf{F}$ that represents the open dimer $\mathbf{7 1}$. Since the coordination sphere on one metal is still unsaturated (coordination number $=3$ ), a fifths $\mathrm{NH}_{3}$ molecule would coordinate to that metal giving structure G. Finally, the addition of a sixth $\mathrm{NH}_{3}$ molecule would be accompanied by a dissociation of the dimeric structure leading to a trisolvated monomer $\mathbf{H}$ that structural motif was identified in ammoniacates 45 and 46.

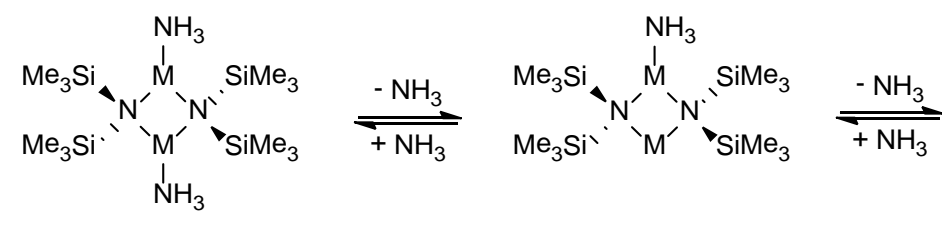

C

B

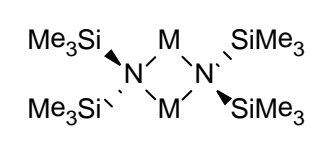

A

$65 \mathrm{M}=\mathrm{Li}$

$66 \mathrm{M}=\mathrm{Na}$

$67 \mathrm{M}=\mathrm{K}$

$68 \mathrm{M}=\mathrm{Rb}$

$$
-\mathrm{NH}_{3} \|+\mathrm{NH}_{3}
$$

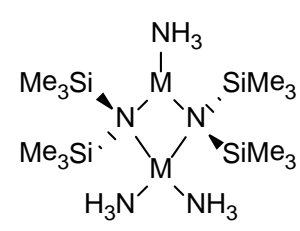

D

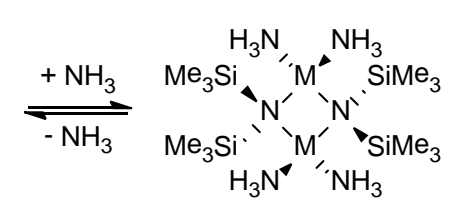

E

$$
\begin{aligned}
& 47 M=K \\
& 48 M=R b
\end{aligned}
$$

$$
\uparrow
$$

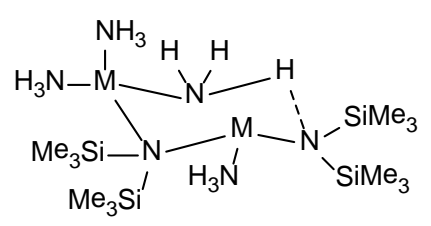

F

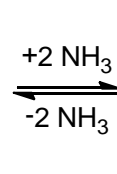<smiles></smiles>

H
$69 \mathrm{M}=\mathrm{Rb}$

$70 \mathrm{M}=\mathrm{Cs}$ 
Scheme 2-2 illustrates the impressive structural diversity of MHMDS ammoniacates in the solid state. Especially the HBs in the monomers and open dimers display a very interesting feature. HBs play a very important role in natural product synthesis. E.g. enzymes take advantage of HBs to achieve highly chemoselective reactions without the necessity of chiral groups on the substrate. This is why it would be very advantageously if the HBs observed in ammoniacates 45, 46 and 71 would still be present in solution. Unfortunately, crystals from the latter are not stable at temperatures higher than $-33^{\circ} \mathrm{C}$. This is why it was not possible to investigate DOSY-ECC-MW-determinations of re-dissolved ammoniacate crystals. Instead, another strategy was used to obtain MHMDS in the presence of ammonia in toluene solution. Therefore, crude MHMDS was solved in TOL- $d_{8}$ (15 mM). Afterwards gaseous $\mathrm{ND}_{3}$ was introduced to the solution (approximately one minute at $+25^{\circ} \mathrm{C}$ ). Finally, the NMR-tubes were sealed and DOSY measurements were performed at RT. DOSY measurements were investigated with Li-, Na- and KHMDS at RT. All ${ }^{1} \mathrm{H}$-signals are highly broadened (Fig. 2-21), indicating that more than one species could be present in solution.

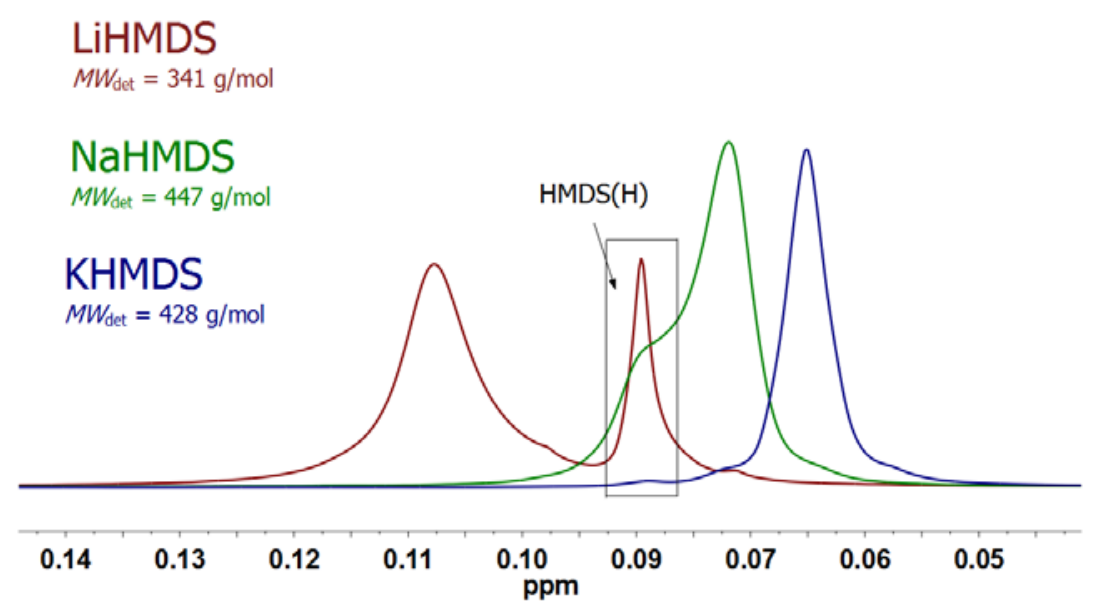

Fig. 2-21. ${ }^{1} \mathrm{H}-\mathrm{NMR}$-superposition plot of MHMDS $(\mathrm{M}=\mathrm{Li}, \mathrm{Na}$ and $\mathrm{K} ; 15 \mathrm{~mm})$ in TOL- $d_{8}$ with ammonia as donor base. The signals are significant broaden, indicating that more than one species could be present in solution.

In the solutions of Li- and NaHMDS there is also a significant concentration of protonated amine $\operatorname{HMDS}(\mathrm{H})$ that could be a results from partial protonation of MHMDS (e.g. via deprotonation of $\mathrm{ND}_{3}$ ). However, DOSY-ECC-MW-determination on LiHMDS predicts a MW of $M W_{\text {det }}=341 \mathrm{~g} / \mathrm{mol}$ giving the hint that LiHMDS could be present as dimeric structure $\mathbf{A}, \mathbf{B}$ or $\mathbf{C}$ with up to two molecules of ammonia $\left(M W_{\mathrm{err}}=-2 \%\right.$ to $7 \%$, A-Table 14 in the appendix). In the case of NaHMDS the determined MW is significantly higher $\left(M W_{\text {det }}=447 \mathrm{~g} / \mathrm{mol}\right.$, A-Table 15 in the appendix $)$. This MW matches with dimeric tri- to 
penta- solvated NaHMDS (D-G, $M W_{\text {err }}=-7 \%$ to $1 \%$ ). This result at least does not rule out the possibility of intramolecular $\mathrm{HBs}$ to be present in solution, since structure $\mathbf{F}$ corresponds to the open dimer $\mathbf{7 1}$ that was observed in the solid state.

The MW-prediction of KHMDS gives a MW of $M W_{\text {det }}=428 \mathrm{~g} / \mathrm{mol}$ that fits to dimeric mono- to- tetra solvated KHMDS (B-E, $M W_{\text {err }}=-3 \%$ to $8 \%$, A-Table 16 in the appendix). Structure $\mathbf{E}$ would confirm the solid state structure of $\left[\left(\mathrm{NH}_{3}\right)_{2} \cdot \mathrm{KN}\left(\mathrm{SiMe}_{3}\right)_{2}\right]_{2}(47)$.

Unfortunately these results have to be considered critically. Several factors could infect the determined MW so several aspects have to be investigated more in detail:

1) Intermolecular HBs between distinct species would increase the MW.

2) It was not cleared if HMDS(H) interacts with MHMDS ammoniacates.

3) Traces of ammonia could react with Li or NaHMDS.

4) The concentration of $\mathrm{NH}_{3}$ and MHMDS should have a significant influence on the solution structures.

5) Low temperature measurements should give further information about the aggregation states. 


\subsubsection{Structure of Hauser Base ${ }^{i} \mathrm{Pr}_{2} \mathrm{NMgCl}$ in THF solution ${ }^{14}$}

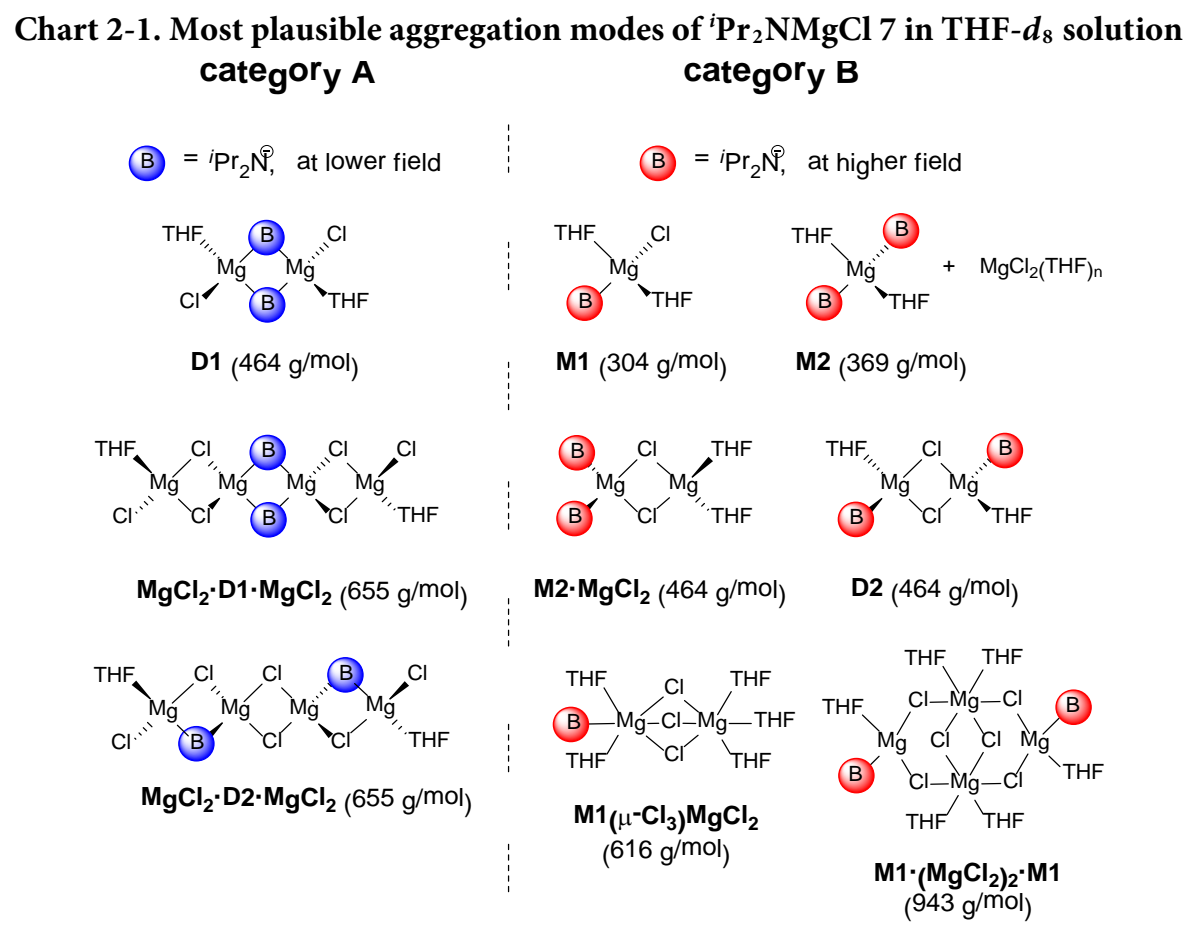

One of the most widely utilized classes of synthetic reagents are Grignard compounds that can be at the simplest level described by "RMgX" (where $\mathrm{R}$ is an organic group and $\mathrm{X}$ a halide). Today especially Grignard reagents with an amido ligand of the type $R_{1} R_{2} N M g X$, so called Hauser bases, and their Turbo derivate $\mathrm{R}_{1} \mathrm{R}_{2} \mathrm{NMgX} \cdot \mathrm{LiCl}$ play a huge role in modern chemistry (see chapter 1.1.3).

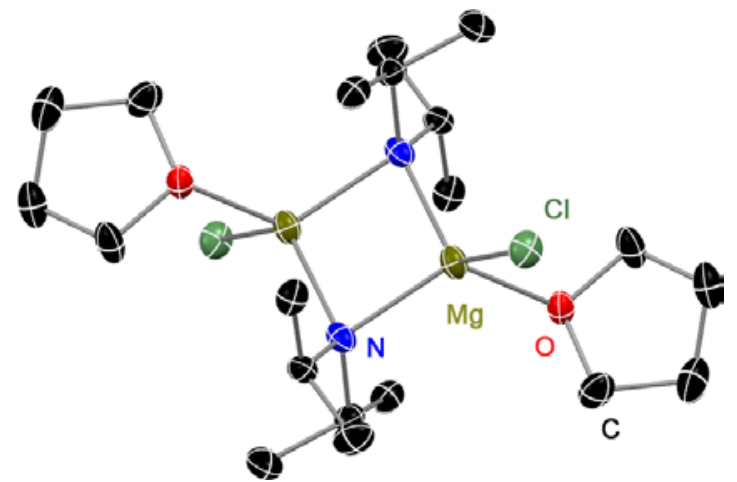

Fig. 2-22. Solid state structure of $[7 \cdot \mathrm{THF}]_{2}$ (D1) with hydrogen atoms and disorder omitted for clarity. Anisotropic displacement parameters are depicted at the $50 \%$ probability level. Selected bond lengths and angles are given in chapter 4.4.6.

\footnotetext{
${ }^{14}$ A revised version of my publication: R. Neufeld, T. L. Teuteberg, R. Herbst-Irmer, R. A. Mata, D. Stalke, JACS 2016, submitted.
} 
However, because of their complex solution behavior, where Schlenk-type equilibria are involved, very little is known about their structure in solution. To shed light on the solution structure of ${ }^{i} \operatorname{Pr}_{2} \mathrm{NMgCl} 7$ first the prominent Hauser base ${ }^{i} \mathrm{Pr}_{2} \mathrm{NMgCl} 7$ was synthesized and crystallized from a THF/toluene $1: 1$ mixture at $-6^{\circ} \mathrm{C}$ (see chapter 0 ). The crystal structure of $[7 \cdot \mathrm{THF}]_{2}$ is shown in Fig. 2-22. TMP $^{[72]}$ and $\operatorname{HMDS}^{[73]}$ Hauser bases alike all Grignard dimers ${ }^{[67 \mathrm{a}, 67 \mathrm{~b}, 67 \mathrm{e}, 74]}$ show the halides in the bridging position in the solid state (like D2, category B in Chart 2-1). In contrast, $[7 \cdot \mathrm{THF}]_{2}$ dimerises featuring the amido ligands in the bridging position (D1, category A in Chart 2-1). Searching the Cambridge Crystallography Database for Hauser bases reveals that there are only three other dimeric amido bridged Hauser bases in the literature. ${ }^{15}$ All have one in common: They feature less bulky amido ligands like $\mathrm{Et}_{2} \mathrm{~N}^{-},{ }^{[73 a]} \mathrm{Ph}_{3} \mathrm{P}=\mathrm{N}^{-[75]}$ and ${ }^{i} \mathrm{Pr}_{2} \mathrm{~N}^{-}$. ${ }^{[28]}$ It can be concluded that in the solid state the switch from the halide to the amido bridge seems advantageous. ${ }^{[64]}$ However, solid state structures may not necessarily be maintained in solution. In synthesis organometallic compounds are predominantly used in solution. Therefore it is highly important to estimate the solution structure of ${ }^{i} \operatorname{Pr}_{2} \mathrm{NMgCl} 7$. The most plausible aggregation modes of a Hauser base in THF solution are demonstrated in Chart 2-1. A dissolved crystal of $[7 \cdot \mathrm{THF}]_{2}$ in THF can on the one hand retain its dimeric status D1 or isomerize to the chloride bridged dimer D2. On the other hand these dimers can dissociate to the monomer M1 or rearrange according to the Schlenk-equilibrium to the diorgano-magnesium $\mathbf{M} 2$ and $\mathrm{MgCl}_{2}$. When an excess of $\mathrm{MgCl}_{2}$ is present, $\mathrm{MgCl}_{2}$ cocoordinated species like $\mathbf{M 1}\left(\boldsymbol{\mu}-\mathrm{Cl}_{3}\right) \mathbf{M g C l}, \quad \mathbf{M} 2 \cdot \mathbf{M g C l}, \quad \mathbf{M g C l}_{2} \cdot \mathbf{D} 1(\mathrm{D} 2) \cdot \mathbf{M g C l}_{2}$ or $\mathbf{M 1} \cdot\left(\mathrm{MgCl}_{2}\right)_{2} \cdot \mathbf{M 1}$ might also be present in solution. All of the mentioned species can be either distinguished by their MW or additionally by the chemical environment of the ${ }^{i} \mathrm{Pr}$ groups that is reflected in the chemical shift $\delta$. Compared to terminal amido ligands (category B, Chart 2-1), bridging ligands (category A, Chart 2-1) show due to the presence of additional electron withdrawing metals a significant low field shift. At RT the ${ }^{1} \mathrm{H}$ NMR spectrum of $[7 \cdot \mathrm{THF}]_{2}$ (Fig. 2-23) shows one broad signal set corresponding to a single species $\mathbf{a} 1$ at high field (2.94/1.01 ppm for $\mathrm{a}-\mathrm{CH} / \mathrm{CH}_{3}$, category B).

\footnotetext{
${ }^{15}$ Cambridge Structural Database CSD, version 5.36 (Updated Nov 2014), chelating amido ligands have been excluded.
} 

Chart 2-1. Most plausible aggregation modes of ${ }^{i} \mathrm{Pr}_{2} \mathrm{NMgCl} 7$ in THF- $d_{8}$ solution
category A
cate $_{\mathrm{g}}$ or $_{\mathrm{y}} \mathrm{B}$
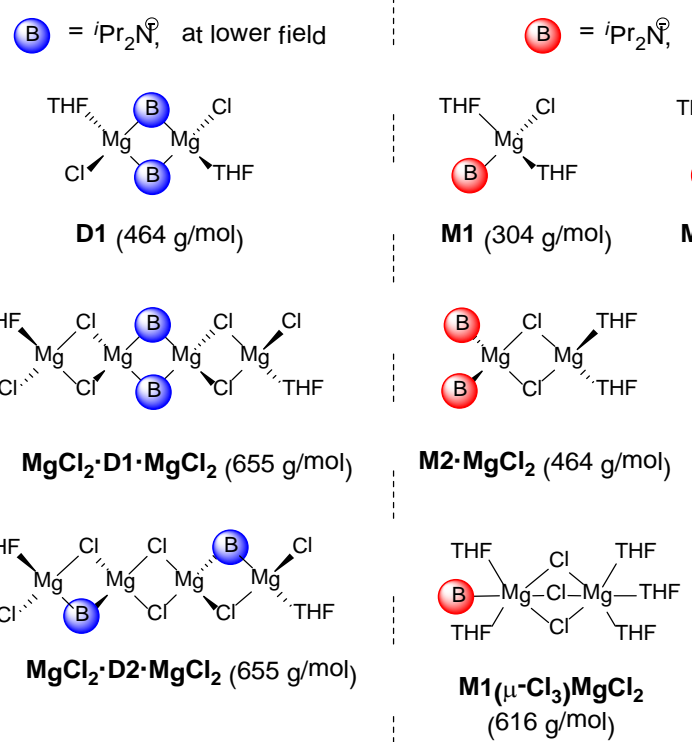
THF THF THF $\mathrm{Cl}-\mathrm{Mg}-\mathrm{Cl}$
(B) Cl-Mg-Cl THF THF THF
$\mathrm{M} 1 \cdot\left(\mathrm{MgCl}_{2) 2} \cdot \mathrm{M} 1\right.$ $(943 \mathrm{~g} / \mathrm{mol})$

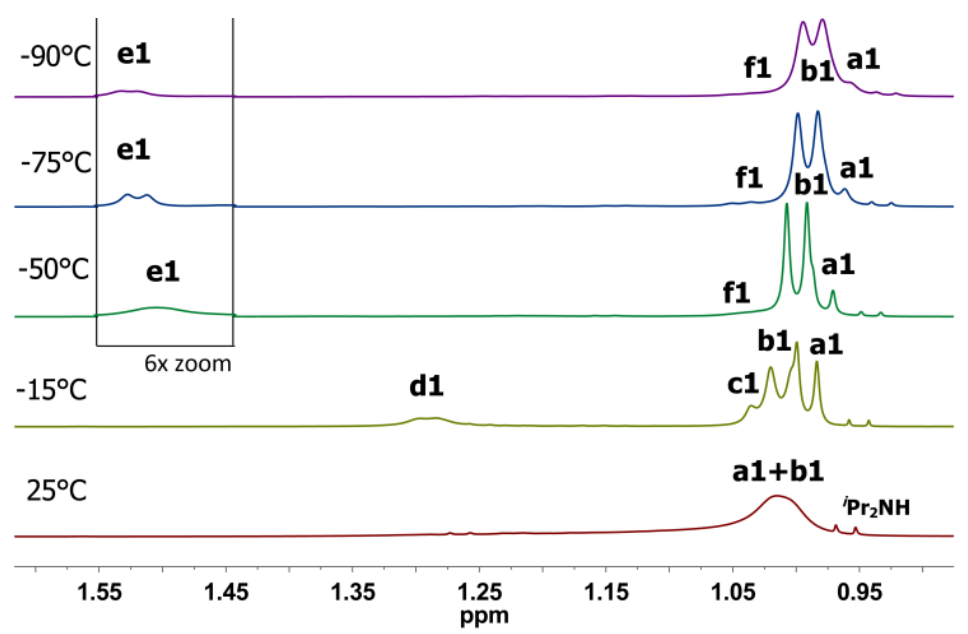

Fig. 2-23. ${ }^{1} \mathrm{H}$ superposition plot of crystalline $[7 \cdot \mathrm{THF}]_{2}\left(0.10 \mathrm{M},-\mathrm{CH}_{3}\right.$ region $)$ re-dissolved in THF- $d_{8}$ at various temperatures. For signal assignment see also Scheme 2-3. A spectrum including the $\alpha-\mathrm{CH}$ region is displayed in A-Figure 4 in the appendix. 
The ${ }^{1} \mathrm{H}$-DOSY-ECC-MW-determination agrees best with the heteroleptic monomer M1 $\left(M W_{\text {calc }}=304 \mathrm{~g} / \mathrm{mol}, M W_{\text {det }}=310 \mathrm{~g} / \mathrm{mol}, \mathrm{MW}_{\text {err }}=-2 \%\right)^{16}$ while homoleptic monomer M2 $\left(M W_{\text {err }}=16 \%\right)$, dimeric $\mathbf{D 2}, \mathbf{M} 2 \cdot \mathbf{M g C l}_{2}$ and bigger aggregates can be easily excluded $\left(M W_{\text {err }} \geq 33 \%\right)$. Below $0^{\circ} \mathrm{C}$ two additional species b1 (3.10/1.02 ppm, category B), c1 (3.24/1.01 ppm, category B) at high field and d1 (3.43/1.29 ppm, category A) at low field appear. From NMR studies on alkyl Grignard reagents it is known that homoleptic dialkylmagnesium monomers $\mathbf{M} 2$ resonance at lower field, compared to heteroleptic monomers M1. ${ }^{[121]}$ In fact, MW-determination for species b1 agree perfectly with the homoleptic diorganomagnesium M2 $\left(M W_{\text {calc }}=369 \mathrm{~g} / \mathrm{mol}, M W_{\text {det }}=356, M W_{\text {err }}=4 \%\right)$. The MW of c1 matches to dimeric D2 and $\mathbf{M} 2 \cdot \mathbf{M g C l}_{2}\left(M W_{\text {calc }}=464 \mathrm{~g} / \mathrm{mol}, M W_{\text {det }}=\right.$ $\left.450 \mathrm{~g} / \mathrm{mol}, M W_{\text {err }}=3 \%\right)$ that have similar MWs. Both have a comparable chemical environment and cannot be distinguished by their MWs. Both species could be present in solution but it is plausible that the equilibrium should be significantly on the side of dimer D2 since it displays a less steric hindrance compared to $\mathbf{M} 2 \cdot \mathbf{M g C l}_{\mathbf{2}}$. In order to investigate the structure of the dimer in solution structure, electronic structure calculations were carried out by Teuteberg and Mata. The latter were performed with the B3LYP-D3 method, ${ }^{[122]}$ including solvent corrections through the use of the COSMO continuum model. ${ }^{[123]}$ Free energy differences confirm $\mathbf{M} \mathbf{2} \cdot \mathbf{M g C l}_{\mathbf{2}}$ to be disfavored relative to the D2 species by $53.1 \mathrm{~kJ} / \mathrm{mol}$ (A-Table 24 in the appendix). The most stable structure found corresponds in fact to a cis-isomer of D2, with both bases orientated to the same side of the $\mathrm{Mg}_{2} \mathrm{Cl}_{2}$ ring. This arrangement optimizes dispersion interactions between both the propyl moieties and the THF rings on each side. The trans configuration is slightly higher in energy by $7.2 \mathrm{~kJ} / \mathrm{mol}$. However, this marginal difference is not to be taken as granted since weak interactions with the solvent (which in our computations is only included as a dielectric continuum) could easily counterbalance this effect. The optimized structure of $\mathbf{M} 2 \cdot \mathbf{M g C l}_{2}$ shows a large $\mathrm{N}-\mathrm{Mg}-\mathrm{N}$ angle of about $146^{\circ}$ illustrating the steric stain of both diisopropylamido groups when coordinated to the same metal center adding to the energetic disfavor (A-Figure 13 in the appendix). Species d1, with the highly low field shifted signal is in good agreement with the amido bridged dimer D1 $\left(M W_{\text {calc }}=464 \mathrm{~g} / \mathrm{mol}\right.$, $\left.M W_{\text {det }}=435 \mathrm{~g} / \mathrm{mol}, M W_{\text {err }}=6 \%\right)$ that is similar to the crystal structure of $[7 \cdot \mathrm{THF}]_{2}$ in Fig. 2-22. Lowering the temperature dramatically influences the Schlenk-equilibrium of Hauser base 7 . While at RT the monomer M1/M2 ratio was 4:1, this ratio switches completely at $-75^{\circ} \mathrm{C}$ (Table 2-11, 0.100 M).

\footnotetext{
${ }^{16}$ All $M W_{\text {det }}$ values are dispayed for each species as an average value, derived from DOSY-ECC-MW measurements at various temperatures, see A-Table 17 in the appendix.
} 

Chart 2-1. Most plausible aggregation modes of ${ }^{i} \mathrm{Pr}_{2} \mathrm{NMgCl} 7$ in THF- $d_{8}$ solution
categor $\mathrm{A}$
cate ${ }_{\mathrm{y}} \mathrm{rr}_{\mathrm{y}} \mathrm{B}$
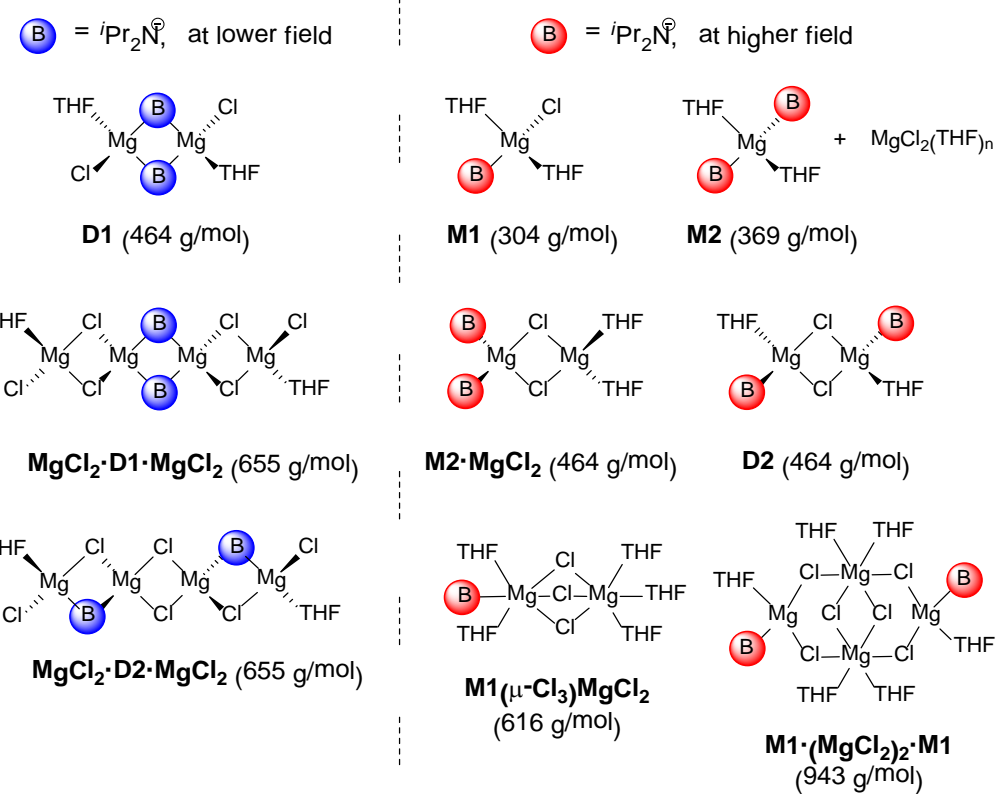

Table 2-11. The Schlenk equilibrium “constant" a) $K_{s}$ for the reaction $\left({ }^{i} \mathrm{Pr}_{2} \mathrm{~N}\right)_{2} \mathrm{Mg}(\mathrm{M} 2)+\mathrm{MgCl}_{2} \rightleftharpoons 2{ }^{i} \operatorname{Pr}_{2} \mathrm{NMgCl}(\mathrm{M1})$

\begin{tabular}{ccc}
\hline $\begin{array}{c}\text { Temp. } \\
{\left[{ }^{\circ} \mathrm{C}\right]}\end{array}$ & $\begin{array}{c}K_{s} \\
(0.015 \mathrm{M})\end{array}$ & $\begin{array}{c}K_{s} \\
(0.100 \mathrm{M})\end{array}$ \\
\hline 25 & 25.00 & 16.00 \\
0 & 8.16 & 4.43 \\
-15 & 2.97 & 1.49 \\
-25 & 1.00 & 0.68 \\
-50 & 0.18 & 0.10 \\
-75 & 0.12 & 0.05
\end{tabular}

a) Usually the Schlenk-equilibrium constant $K_{\mathrm{s}}$ is derived from ${ }^{1} \mathrm{H}$ integrals of $\alpha-\mathrm{CH}$ protons with $K_{\mathrm{s}}=$ $\left[{ }^{i} \mathrm{Pr}_{2} \mathrm{NMgCl}\right]^{2} /\left[\left({ }^{i} \mathrm{Pr}_{2} \mathrm{~N}\right)_{2} \mathrm{Mg}\right]^{2}$ with the approximation: $\left[\left({ }^{i} \mathrm{Pr}_{2} \mathrm{~N}\right)_{2} \mathrm{Mg}\right] \approx\left[\mathrm{MgCl}_{2}\right]$. However, our results show that the concentration of $\left({ }^{i} \operatorname{Pr}_{2} \mathrm{~N}\right)_{2} \mathrm{Mg}$ is not equal to $\mathrm{MgCl}_{2}$ because the latter is involved in further reactions with Hauser base 7 . This is also reflected in our determined $K_{\mathrm{s}}$ values that show therefore a concentration dependency. 
The huge population of homoleptic monomer $\mathbf{M} 2$ at low temperatures stays in good agreement with the work of Smith and Becker who showed that the formation of $\mathrm{RMgCl}$ from $\mathrm{R}_{2} \mathrm{Mg}$ and $\mathrm{MgCl}_{2}$ is endothermic in THF solution. ${ }^{[69]}$ Same seems to be true for Hauser base 7. The equilibrium constants of the Schlenk-equilibrium are summarized in Table 2-11, showing that the equilibrium moves to the side of homoleptic $\mathbf{M} 2+\mathrm{MgCl}_{2}$ with increasing concentration and decreasing temperature. The formation of M2 is accompanied by the release of $\mathrm{MgCl}_{2}$ to the solution, most probably as a monomer with up to four THF molecules. ${ }^{[124]}$ Therefore it might not be surprising when free $\mathrm{MgCl}_{2}$ coordinates to some complexes at low temperatures. At $-50^{\circ} \mathrm{C}$ dimers D1 (d1) and D2 (c1) dissipate and two new species el (3.39/1.50 ppm, category A) and f1 (3.21/ $1.04 \mathrm{ppm}$, category B) that have an even more low field shift appears. This intense shift can be attributed to an additional coordination of $\mathrm{MgCl}_{2}$ to $\mathbf{M 1}, \mathbf{D 1}$ or D2. When magnesium chloride coordinates to monomer $\mathbf{M 1}$ than a structure like $\mathbf{M 1}\left(\boldsymbol{\mu}-\mathbf{C l}_{3}\right) \mathbf{M g C l}_{\mathbf{2}}$ (Chart 2-1, category B) would be feasible. This coordination mode was suggested for methyl magnesium chloride by Sakamoto and Imamoto et al. ${ }^{[125]}$ With the help of coldspray ionization mass spectrometry (CSI-MS), they proposed that the $\mu-\mathrm{Cl}_{3}$ bridged Grignard reagent was coordinated by four to six THF molecules, whereas the species with five THF's was the major component. ${ }^{[125]}$ Additional support is given by several crystal structures of cationic $\left[(\mathrm{THF})_{3} \mathrm{Mg}\left(\mu-\mathrm{Cl}_{3}\right) \mathrm{Mg}(\mathrm{THF})_{3}\right]^{+}$where $\mathrm{MgCl}_{2}$ is also coordinated in that $\mu-\mathrm{Cl}_{3}$ coordination mode. ${ }^{[125]}$ The DOSY-ECC-MW-investigation shows similar results: At $-70^{\circ} \mathrm{C}$ the MW-determination gives for signal f1 a MW of $M W_{\text {det }}=512 \mathrm{~g} / \mathrm{mol}$ that fits to $\mathbf{M 1}\left(\boldsymbol{\mu}-\mathbf{C l}_{3}\right) \mathbf{M g C l}_{2}$ with four THF molecules $\left(M W_{\text {calc }}=544 \mathrm{~g} / \mathrm{mol}, M W_{\text {det }}=512 \mathrm{~g} / \mathrm{mol}\right.$, $\left.M W_{\text {err }}=6 \%\right)$. At $-80^{\circ} \mathrm{C}$ the $\mathrm{MW}$ increases significantly to $M W_{\mathrm{det}}=616 \mathrm{~g} / \mathrm{mol}$ that matches perfectly with the THF-five-fold solvated $\mathbf{M 1}\left(\boldsymbol{\mu}-\mathbf{C l}_{3}\right) \mathbf{M g C l}_{2}\left(M W_{\text {calc }}=616 \mathrm{~g} / \mathrm{mol}, M W_{\text {det }}=\right.$ $616 \mathrm{~g} / \mathrm{mol}, M W_{\text {err }}=0 \%$ ), indicating that a higher solvation is favoured at lower temperatures. Unfortunately, below $-80^{\circ} \mathrm{C}$ the signal was too low in intensity for further MW-determination investigations. In the literature there are several crystal structures of the type M1·( $\left.\mathbf{M g C l}_{2}\right)_{2} \cdot \mathbf{M 1}$ (Chart 2-1, category B), where two monomers $\mathbf{M 1}$ are bridged by two magnesium dichlorides in an open cubic aggregation mode. ${ }^{[125-126]}$ However, in solution of 7 it seems that the dissociation into smaller parts is favoured over an open cubic arrangement $\left(M W_{\text {calc }}=943 \mathrm{~g} / \mathrm{mol}, M W_{\text {det }}=616 \mathrm{~g} / \mathrm{mol}, M W_{\text {err }}=35 \%\right)$, which was already proposed by D. Seyferth in 2009. ${ }^{[71]}$ 
Additionally to f1, species el appears at lower field (category A). The DOSY-ECC-MWdetermination shows a temperature dependent MW distribution $\left(M W_{\text {det }}=409 \mathrm{~g} / \mathrm{mol}\right.$ at $-50^{\circ} \mathrm{C}, 446 \mathrm{~g} / \mathrm{mol}$ at $-70^{\circ} \mathrm{C}$ and $578 \mathrm{~g} / \mathrm{mol}$ at $-80^{\circ} \mathrm{C}$ ). The addition of $\mathrm{MgCl}_{2}$ to dimers $\mathbf{D 1}$ or D2 would produce aggregates like $\mathbf{M g C l}_{2} \cdot \mathbf{D} 1 \cdot \mathbf{M g C l}_{\mathbf{2}}$ or $\mathbf{M g C l}_{2} \cdot \mathbf{D} 2 \cdot \mathbf{M g C l}_{\mathbf{2}}$ (Chart 2-1, category A). However, several other $\mathrm{MgCl}_{2}$ coordinated, amido bridged species would also be thinkable. Unfortunately, in the literature there are no crystal structures of $\mathrm{MgCl}_{2}$ coordinated, ligand bridged Hauser bases or Grignard reagents that indicate the most plausible aggregate. The high $\mathrm{MW}$ of $578 \mathrm{~g} / \mathrm{mol}$ at $-80^{\circ} \mathrm{C}$ gives much room for interpretation. This is why we can only speculate how the composition of aggregate e1 could look like. Noticeable is that in contrast to all other species, the $-\mathrm{CH}_{3}$ signal of e1 shifts to lower field with lower temperature (Fig. 2-23). This behaviour could be a result of a successive addition of $\mathrm{MgCl}_{2}$ to $\mathrm{D} 1$ or $\mathbf{D} 2$. At $-80^{\circ} \mathrm{C}$ the shift to lower field stops and the MW of $\mathbf{e l}$ matches with dimeric $\left.\mathbf{M g C l}_{2} \cdot \mathbf{D 1} \mathbf{( D 2}\right) \cdot \mathbf{M g C l}_{2}\left(M W_{\text {calc }}=655 \mathrm{~g} / \mathrm{mol}, M W_{\text {det,corr }}=\right.$ $703 \mathrm{~g} / \mathrm{mol}, M W_{\text {err }}=-7 \%$, after molar density correction due to highly increased molar van der Waals density $\left(M D_{\mathrm{W}}\right)$ of this species, see A-Table 21 and A-Table 22 in the appendix). However, like already mentioned, this MW-agreement has to be considered with caution. Anyway, it seems that at low temperatures free, monomeric $\mathrm{MgCl}_{2}$ is disadvantageous in solution of Hauser base 7. Instead, the coordination of $\mathrm{MgCl}_{2}$ to monomeric and/or dimeric $\mathrm{RMgCl}$ molecules should be favoured like it is e.g. the case for $\mathrm{LiCl}$ that will be discussed in the following chapter. 


\subsubsection{Structure of Turbo-Hauser Base ${ }^{i} \mathrm{Pr}_{2} \mathrm{NMgCl} \cdot \mathrm{LiCl}$ in THF solution $^{17}$}

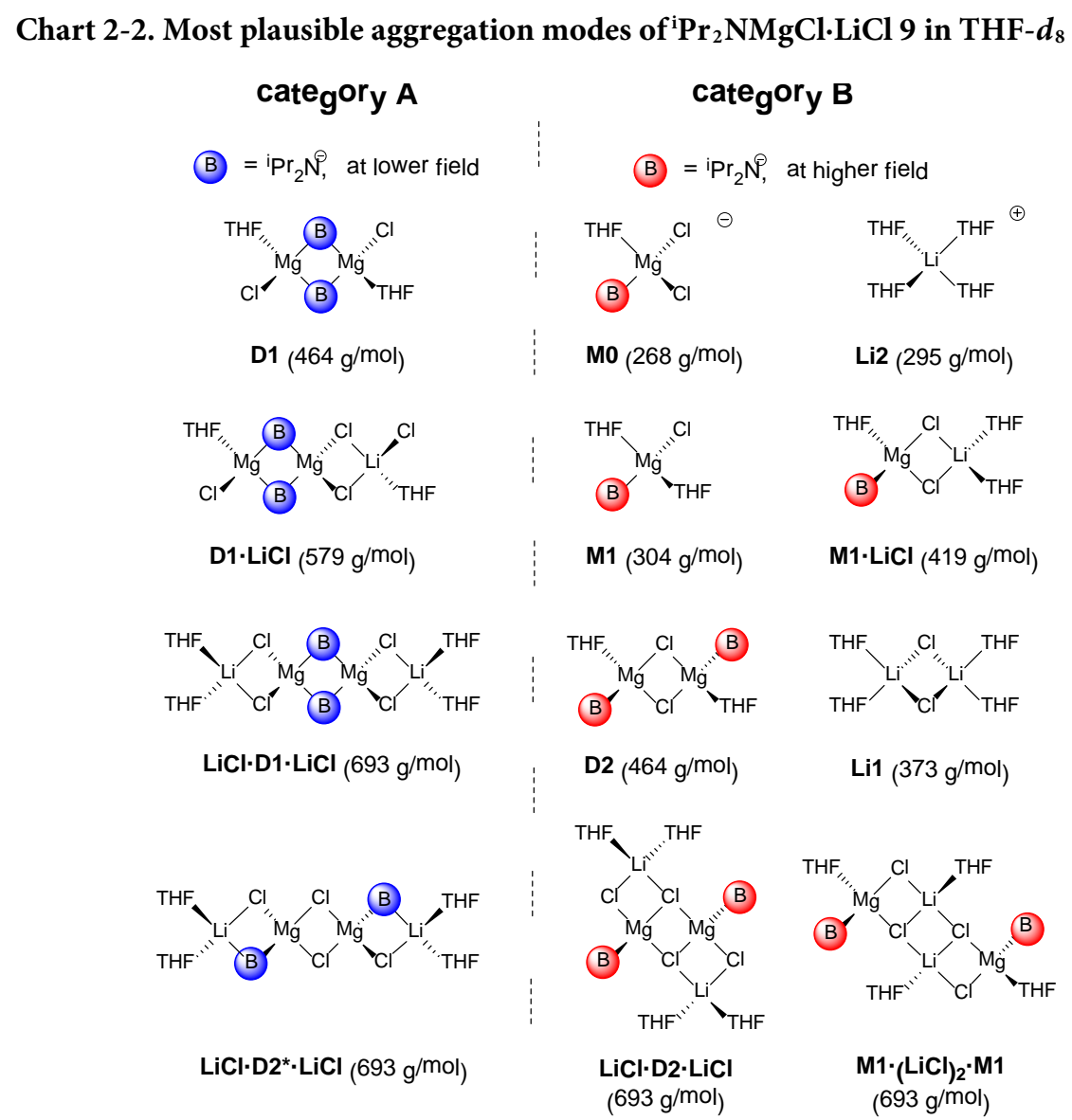

The impact of $\mathrm{LiCl}$ on the solution structure of Grignard reagents and Hauser bases is still vigorously discussed. Knochel et al. suggest that $\mathrm{LiCl}$ deaggregates $\mathrm{RMgX}$ oligomers ${ }^{[23]}$ and forms a more reactive bimetallic monomer $\mathrm{RMgCl} \cdot \mathrm{LiCl}$ that is supposed to furnish magnesiate character to the Grignard reagent in the sense of a solvent separated ion pair (SSIP) $\left[\mathrm{Li}(\mathrm{THF})_{4}\right]^{+}\left[\mathrm{RMg}(\mathrm{THF}) \mathrm{Cl}_{2}\right]^{-} \cdot{ }^{[76]}$ Crystallographic evidence of the Turbo-Hauser bases $\mathrm{TMPMgCl} \cdot \mathrm{LiCl}^{[72]}\left(\mathbf{M 1} \cdot \mathbf{L i C l}\right.$, Chart 2-2 with $\left.\mathrm{B}=\mathrm{TMP}^{-}\right)$and ${ }^{i} \mathrm{Pr}_{2} \mathrm{NMgCl} \mathrm{LiCl}^{[28]} 9$ (LiCl-D1·LiCl, Chart 2-2) supports the contact ion pair (CIP) coordination mode in the solid state. But still it was not clear whether the mixed metal structure really is maintained in solution or just a transient species. García-Álvarez and Mulvey et al. analyzed ${ }^{[28]}$ crystals of $[9 \cdot \mathrm{THF}]_{2}$ in THF- $d_{8}$ solution at $-50^{\circ} \mathrm{C}$ by employing the internal calibration curve (ICC) D-FW analysis that was pioneered by $L i$ and Williard et al. ${ }^{[77]}$ Because of the lack of

\footnotetext{
${ }^{17}$ A revised version of my publication: R. Neufeld, T. L. Teuteberg, R. Herbst-Irmer, R. A. Mata, D. Stalke, JACS 2016, submitted.
} 
appropriate references the accuracy of the method was not sufficient and they concluded that they were not able to "clearly establish the exact nature of the solution species". ${ }^{[28]}$ However, the first key conclusion was that the molecular structure of crystalline $[\mathbf{9} \cdot \mathrm{THF}]_{2}$ $(\mathbf{L i C l} \cdot \mathbf{D 1} \cdot \mathbf{L i C l})$ was not retained in THF- $d_{8}$ solution and the second was that a SSIP situation like it was proposed by Knochel, described by negative charged magnesium ate complexes (like e.g. M0) and free $\left[\mathrm{Li}(\mathrm{THF})_{4}\right]^{+}(\mathbf{L i 2})$ seemed most probable (category B in Chart 2-2). ${ }^{[28]}$ In this chapter some light will be shed on the complex solution structure of 9. Additionally, it will be shown that both above mentioned key conclusions have to be revised. Again the DOSY-ECC-MW-determination hand-in-hand with theoretical calculations will be applied to clarify the influence of $\mathrm{LiCl}$ on the Schlenk-equilibrium of 7 .

Primarily it seems advisable to a priori rationalize which species are feasible to be present in the solution of ${ }^{i} \mathrm{Pr}_{2} \mathrm{NMgCl} \cdot \mathrm{LiCl}$ 9. A dissolved crystal of $[9 \cdot \mathrm{THF}]_{2}$ in THF can either retain the coordination $\mathbf{L i C l} \cdot \mathbf{D} \mathbf{1} \cdot \mathbf{L i C l}$ or isomerize to $\mathbf{L i C l} \cdot \mathbf{D} 2^{\star} \cdot \mathbf{L i C l} \mathbf{L i C l} \cdot \mathbf{D} 2^{*} \cdot \mathbf{L i C l}(\mathrm{Chart}$ 2 -2). In the first aggregation mode the ${ }^{i} \mathrm{Pr}$-groups are neighboured by compact chloride ligands, enabling a free rotation of the alkyl groups, whereas in the latter they are neighboured to a sterically demanding THF molecule that would result in a steric repulsion. This is why species $\mathbf{L i C l} \cdot \mathbf{D 1} \cdot \mathbf{L i C l}$ should be highly favoured over $\mathbf{L i C l} \cdot \mathbf{D} 2^{\star} \cdot \mathbf{L i C l}$. That is again supported by calculations (A-Table 25 in the appendix). The free energy difference between $\mathbf{L i C l} \cdot \mathbf{D 1} \cdot \mathbf{L i C l}$ and $\mathbf{~ L i C l} \cdot \mathbf{D} 2^{\star} \cdot \mathbf{L i C l}$ is $55-56 \mathrm{~kJ} / \mathrm{mol}$ (the former being more stable) in the temperature range from $-90^{\circ} \mathrm{C}$ to $+25^{\circ} \mathrm{C}$. Inspection of the optimized structures confirms the steric hindrance (A-Figure 17 in the appendix). In the LiCl-D1.LiCl structure the $\mathrm{Mg}^{2+}$ cations are aligned with the $\mathrm{Li}^{+}$, resulting in a highsymmetric structure with a close to tetrahedral coordination at each metal atom. In the $\mathbf{L i C l} \cdot \mathbf{D} 2 * \cdot \mathbf{L i C l}$ case, the $\mathrm{Li}^{+}$cations are forced out of this axis in order to accommodate the isopropyl groups at both ends. Another possibility to consider is that the dimer of $[\mathbf{9} \cdot \mathrm{THF}]_{2}$ can dissociate into monomeric units M1.LiCl. These monomers could recombine via a four membered $\mathrm{Mg}_{2} \mathrm{Cl}_{2}$ ring $(\mathbf{L i C l} \cdot \mathbf{D} 2 \cdot \mathbf{L i C l})$ or by a smaller $\mathrm{Li}_{2} \mathrm{Cl}_{2} \operatorname{ring}\left(\mathbf{M l} \cdot(\mathbf{L i C l})_{2} \cdot \mathbf{M 1}\right)$ in the centre. It is also possible that $\mathrm{LiCl}$ dissociates as a well-known dimer ${ }^{[127]}$ $\left[(\mathrm{THF})_{2} \mathrm{Li}(\mu \mathrm{Cl})_{2} \mathrm{Li}(\mathrm{THF})_{2}\right]$ Lil to produce $\mathrm{LiCl}$-free species M1, D1 or D2 or that lithium dissociates as a solvent separated ion pair $\mathrm{Li}(\mathrm{THF}){ }_{4}{ }^{+} \mathbf{L i} 2$ that would produce an SSIP atecomplex like e.g. M0, where two chlorides are coordinated to the magnesium ion (Chart 22). 
Chart 2-2. Most plausible aggregation modes of ${ }^{\mathrm{i}} \mathrm{Pr}_{2} \mathrm{NMgCl} \cdot \mathrm{LiCl} 9$ in THF- $d_{8}$ solution. category A cate or $_{\mathbf{B}} \mathrm{B}$

(B) $=\operatorname{ipr}_{2} \mathrm{~N}$, at lower field

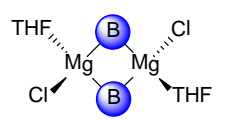

D1 $(464 \mathrm{~g} / \mathrm{mol})$
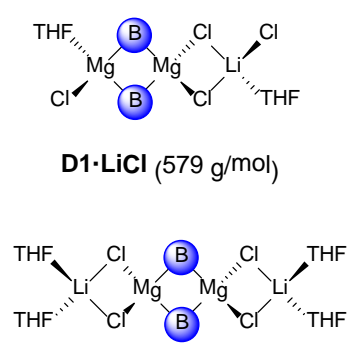

LiCl·D1· LiCl $(693 \mathrm{~g} / \mathrm{mol})$

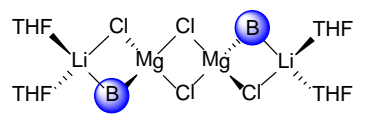

LiCl·D2* LiCl $(693 \mathrm{~g} / \mathrm{mol})$
(B) $={ }^{i} r_{2} \mathrm{~N}$, at higher field

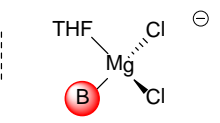

M0 (268 g/mol)

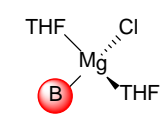

M1 (304 g/mol)

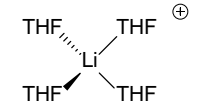

Li2 (295 g/mol)

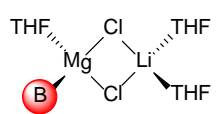

M1- LiCl (419 g/mol)

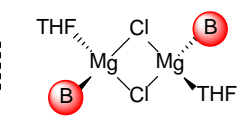

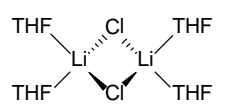

D2 (464 g/mol)<smiles>[TeH-][Te][IH+]</smiles>

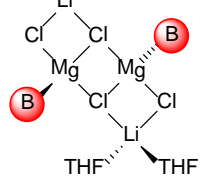

LiCl'D2'LiCl (693 $\mathrm{g} / \mathrm{mol}$ )
Li1 (373 g/mol)

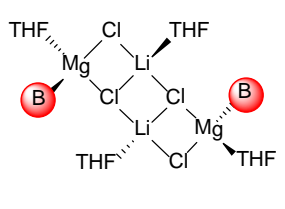

$\mathrm{M1} \cdot(\mathrm{LiCl})_{2} \cdot \mathrm{M} 1$

(693 $\mathrm{g} / \mathrm{mol})$

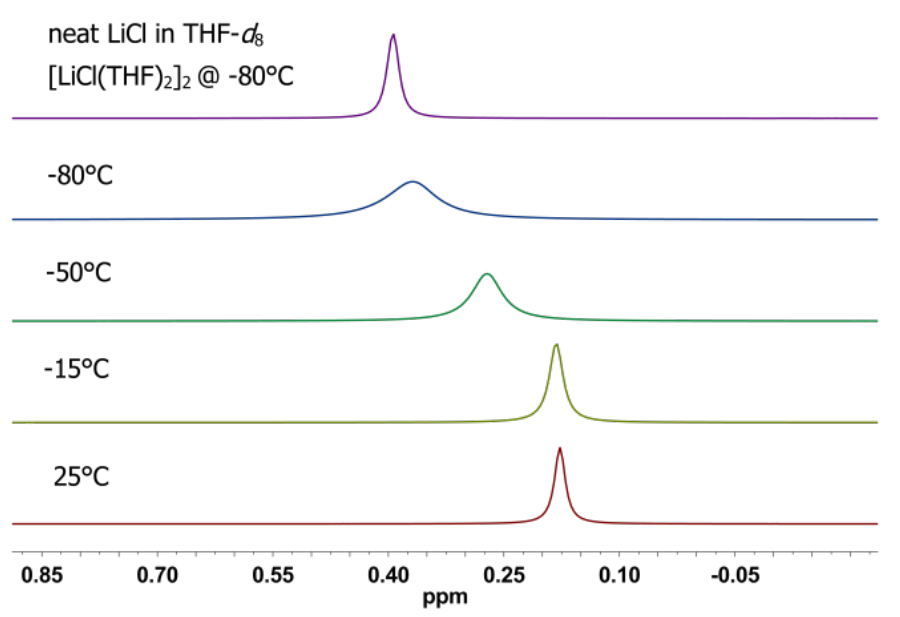

Fig. 2-24. ${ }^{7} \mathrm{Li}$ superposition plot of crystalline $9(0.1 \mathrm{M})$ re-dissolved in THF- $d_{8}$ at various temperatures. On the top: ${ }^{7} \mathrm{Li}$-spectrum of neat $\mathbf{L i 1}$ in THF- $d_{8}$ at $-80^{\circ} \mathrm{C}$. 
The ${ }^{7} \mathrm{Li}$ spectra show at RT a singlet at $0.18 \mathrm{ppm}\left(25^{\circ} \mathrm{C}\right)$ that shifts to lower field with decreasing temperature $\left(0.38 \mathrm{ppm}\right.$ at $-100^{\circ} \mathrm{C}$, Fig. $\left.2-24\right)$. The presence of LDA, where lithium coordinates directly to the diisopropyl amide, can therefore be excluded since LDA resonances in the ${ }^{7} \mathrm{Li}$ NMR experiment temperature independently at about $2.0 \mathrm{ppm}$ (AFigure 11 in the appendix). Additionally, the solvent separated cation $\left[\mathrm{Li}(\mathrm{THF})_{4}\right]^{+} \mathbf{L i} 2$ can be excluded to be populated at detactable concentrations too, since it is known that the solvent separated lithium ion $\mathbf{L i} 2$ resonances predominately at a negative chemical shift $\left(-1.1 \mathrm{ppm}\right.$ in THF). ${ }^{[128]}$ Further support is given by the crystal structure of 9 where the lithium cation is located near to the ${ }^{i} \mathrm{Pr}$-groups in a middle distance of $4.49 \AA$ to the closest $-\mathrm{CH}_{3}$-protons. ${ }^{[72]}$ When lithium is coordinating next to the magnesium amide than such relatively close distance should be visible in a ${ }^{1} \mathrm{H}-{ }^{7} \mathrm{Li}-\mathrm{HOESY}$ experiment. In fact the ${ }^{1} \mathrm{H}$ ${ }^{7} \mathrm{Li}-\mathrm{HOESY}$-spectra show at high temperature a cross peak between the ${ }^{7} \mathrm{Li}$ signal and the $-\mathrm{CH}_{3}$ signals of species $\mathbf{a} 2, \mathbf{b} 2$ and $\mathbf{c 2}$, indicating that lithium does coordinate to Hauser base 7 (Fig. 2-25).

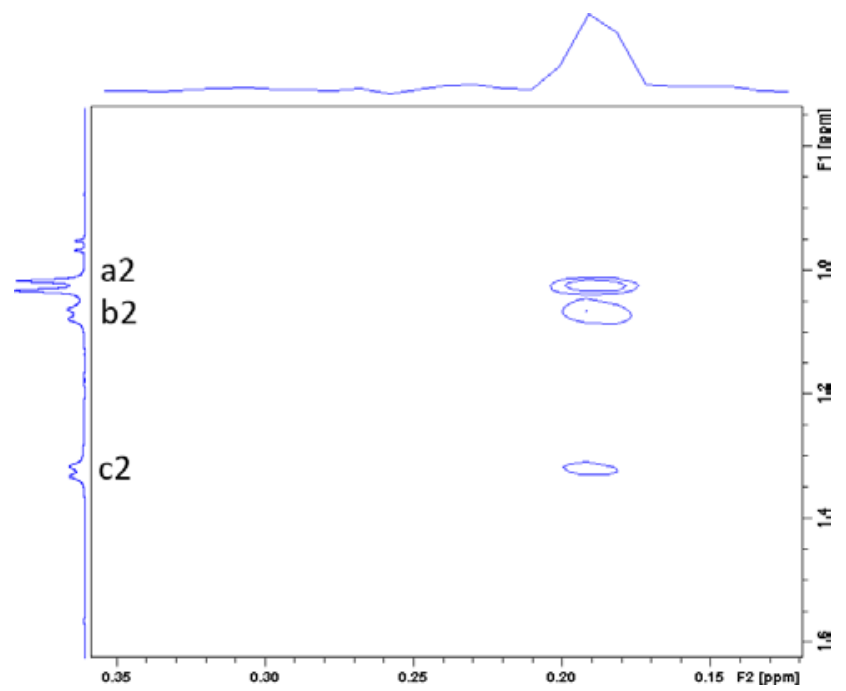

Fig. 2-25. ${ }^{1} \mathrm{H}-{ }^{7} \mathrm{Li}-\mathrm{HOESY}$ of crystalline $9(0.03 \mathrm{M})$ re-dissolved in THF- $d_{8}$ at $0^{\circ} \mathrm{C}$. 


\section{Chart 2-2. Most plausible aggregation modes of ${ }^{\mathrm{i}} \mathrm{Pr}_{2} \mathrm{NMgCl} \cdot \mathrm{LiCl} 9$ in THF- $d_{8}$ solution.} category A

(B) $={ }^{i} \mathrm{Pr}_{2} \mathrm{~N}$, at lower field

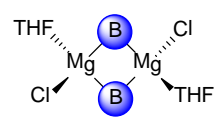

D1 $(464 \mathrm{~g} / \mathrm{mol})$

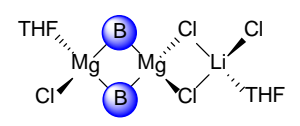

D1. $\mathrm{LiCl}(579 \mathrm{~g} / \mathrm{mol})$

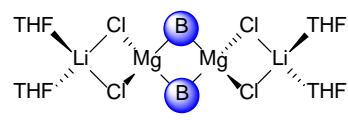

LiCl·D1· LiCl $(693 \mathrm{~g} / \mathrm{mol})$

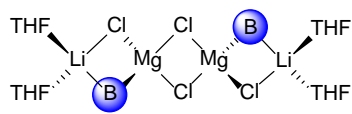

LiCl·D2*' $\mathrm{LiCl}(693 \mathrm{~g} / \mathrm{mol})$

\section{category $B$}

(B) $={ }^{2} \mathrm{Pr}_{2} \mathrm{~N}$, at higher field
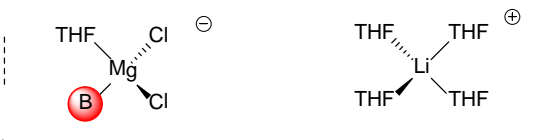

M0 (268 g/mol)

Li2 (295 g/mol)

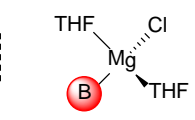

M1 (304 g/mol)

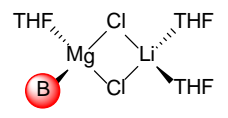

M1- LiCl (419 $\mathrm{g} / \mathrm{mol})$
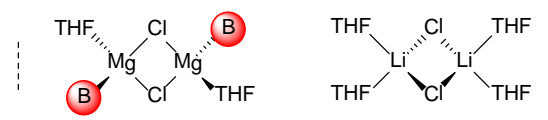

D2 $(464 \mathrm{~g} / \mathrm{mol})$

Li1 (373 g/mol)
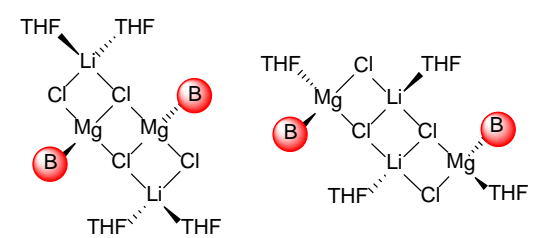

LiCl·D2· LiCl (693 $\mathrm{g} / \mathrm{mol})$
M1·( LiCl $_{2} \cdot \mathrm{M} 1$

$(693 \mathrm{~g} / \mathrm{mol})$

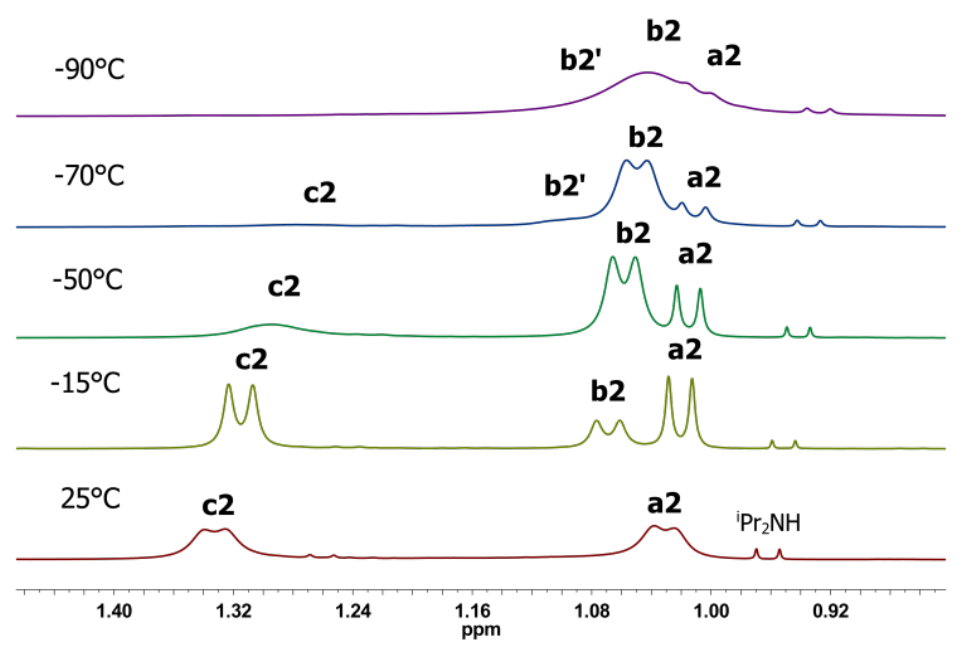

Fig. 2-26. ${ }^{1} \mathrm{H}$ superposition plot of crystalline $9\left(0.1 \mathrm{M},-\mathrm{CH}_{3}\right.$ region) re-dissolved in THF- $d_{8}$ at various temperatures. For signal assignment see also Scheme 2-6. A spectrum including the $\mathrm{a}-\mathrm{CH}$ signals is displayed in A-Figure 6 in the appendix. 
At RT two different species (a2: 2.92/1.03 ppm, category B and c2: 3.42/1.33 ppm, category A) can be identified in the ${ }^{1} \mathrm{H}$ NMR spectrum of 9 (Fig. 2-26). The MW-determination of a2 gives at all temperatures a $\mathrm{MW}$ that fits perfectly to the $\mathrm{LiCl}$ coordinated monomer M1.LiCl $\left(M W_{\text {calc }}=419 \mathrm{~g} / \mathrm{mol}, M W_{\text {det }}=425 \mathrm{~g} / \mathrm{mol}, M W_{\text {err }}=-2 \%\right)$. Compared with the salt free monomer M1 (a1), the metal chloride co-coordinated monomers $\mathbf{M 1}\left(\boldsymbol{\mu}-\mathbf{C l}_{3}\right) \mathbf{M g C l}_{\mathbf{2}}$ (f1) and M1.LiCl (a2) are shifted to lower field $(\Delta \delta \approx 0.02 \mathrm{ppm})$, due to an additional coordination of electron withdrawing metal chloride. The same is true for signal $\mathbf{c} 2$ that appears at the dimer region of D1 (category A, Chart 2-2) but also low field shifted by $0.02 \mathrm{ppm}$ (compared to $\mathrm{LiCl}-$ free dimer D1) indicating that $\mathrm{c} 2$ corresponds to $\mathrm{LiCl}$ cocoordinated dimer LiCl.D1.LiCl. At $25^{\circ} \mathrm{C}$ the determined $\mathrm{MW}$ of $\mathbf{c 2}\left(M W_{\text {calc }}=693 \mathrm{~g} / \mathrm{mol}\right.$, $\left.M W_{\text {det }}=525 \mathrm{~g} / \mathrm{mol}, M W_{\text {err }}=24 \%\right)$ is smaller than expected. However, with decreasing temperature the MW grows significantly $\left(M W_{\text {det }}=598 \mathrm{~g} / \mathrm{mol}\right.$ at $-15^{\circ} \mathrm{C}, 618 \mathrm{~g} / \mathrm{mol}$ at $-40^{\circ} \mathrm{C}$ and $635 \mathrm{~g} / \mathrm{mol}$ at $\left.-50^{\circ} \mathrm{C}\right)$ till it stops growing further at $-60^{\circ} \mathrm{C}\left(M W_{\text {det }}=661 \mathrm{~g} / \mathrm{mol}\right)$. The same trend was already observed for species $\mathbf{M g C l}_{\mathbf{2}} \cdot \mathbf{D 1}$ (D2) $\cdot \mathbf{M g C l}_{\mathbf{2}}$ (e1) of $\mathrm{LiCl}$-free Hauser base 7. In contrast to all other species of Turbo-Hauser base $\mathbf{9}$, the $\mathrm{a}-\mathrm{CH}$ signal of c2 shifts like e1 to lower field with lower temperature (A-Figure 6 in the appendix). This is why a successive coordination of $\mathrm{LiCl}$ to $\mathrm{D} 1$ could again explain this behaviour $\left(25^{\circ} \mathrm{C}\right.$ to $-40^{\circ} \mathrm{C}$ : av $\left.. M W_{\text {det }}=582 \mathrm{~g} / \mathrm{mol}, M W_{\text {calc }}(\mathbf{D 1} \cdot \mathbf{L i C l})=579 \mathrm{~g} / \mathrm{mol}, M W_{\text {err }}=-1 \%\right) .{ }^{18}$ Finally between $-60^{\circ} \mathrm{C}$ and $-70^{\circ} \mathrm{C}$ it is possible to determine the $\mathrm{MW}$ of tetra nuclear dimer LiCl.D1.LiCl with two lithium chlorides coordinated to dimer D1 $\left(M W_{\text {calc }}=693 \mathrm{~g} / \mathrm{mol}\right.$, $\left.M W_{\text {det }}=661 \mathrm{~g} / \mathrm{mol}, M W_{\text {err }}=5 \%\right)$. A monomer-dimer equilibrium of $\mathbf{a} 2$ and $\mathbf{c} 2$ was already suggested by García-Álvarez and Mulvey et al. ${ }^{[28]}$ Concentration experiments showed that species a2 dominates at lower concentrations whereas $\mathbf{c 2}$ is mostly populated at higher concentrations. ${ }^{[28]}$ Below $-70^{\circ} \mathrm{C}$ the solubility limit is reached and signal c2 disappears. With decreasing temperature a third species next to $\mathbf{a} 2$ is forming (b2: 3.13/1.07 ppm, category B). Further cooling results in a shift of the oligomer equilibrium. The integral of $\mathbf{b} 2$ increases significantly at the expense of $\mathbf{a} 2$ and $\mathbf{c} 2$. The MW-determination of $\mathbf{b} \mathbf{2}$ offers at all temperatures a MW that fits to the $\mathrm{LiCl}$ free dimer D2 $\left(M W_{\text {calc }}=464 \mathrm{~g} / \mathrm{mol}, M W_{\text {det }}=\right.$ $\left.461 \mathrm{~g} / \mathrm{mol}, M W_{\text {err }}=1 \%\right)$.

\footnotetext{
${ }^{18}$ This behaviour could be also attributed to a fast exchange of $\mathbf{L i C l} \cdot \mathbf{D} \mathbf{1} \cdot \mathbf{L i C l}$ with its lithium free counterpart D2 or to a dissociation into monomeric M1.LiCl. Both equilibria would produce a smaller effective MW.
} 
Chart 2-2. Most plausible aggregation modes of ${ }^{\mathrm{i}} \mathrm{Pr}_{2} \mathrm{NMgCl} \cdot \mathrm{LiCl} 9$ in THF- $d_{8}$ solution.
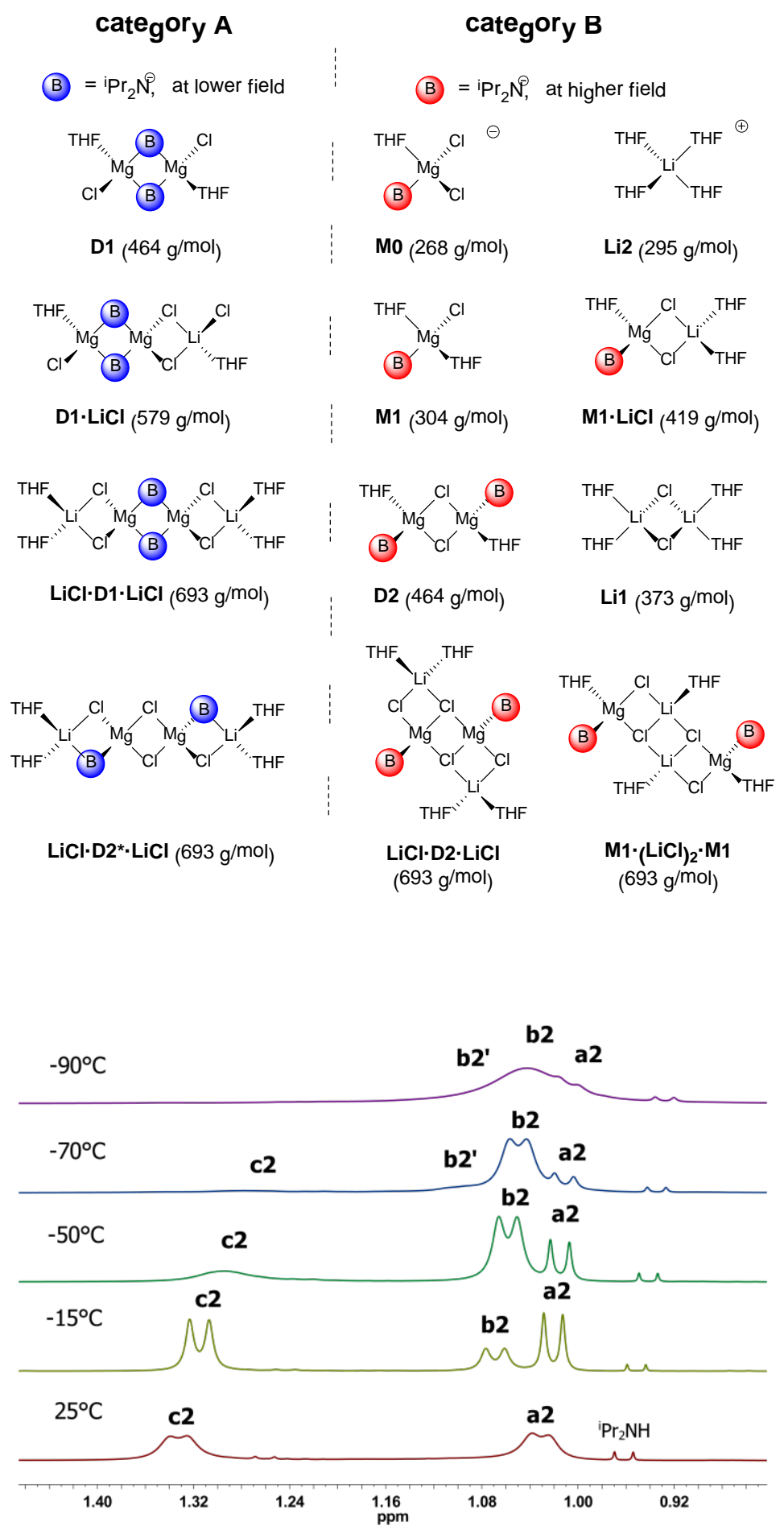

Fig. 2-26. ${ }^{1} \mathrm{H}$ superposition plot of crystalline $9\left(0.1 \mathrm{M},-\mathrm{CH}_{3}\right.$ region $)$ re-dissolved in THF- $d_{8}$ at various temperatures. 
The ${ }^{1} \mathrm{H}-{ }^{7} \mathrm{Li}$ HOESY experiment indicates at $0^{\circ} \mathrm{C}$ the interaction of $\mathbf{b} 2$ with the lithium cation. However, the cross peak vanishes at lower temperatures when $\mathbf{b} \mathbf{2}$ becomes the most populated species, indicating that $\mathbf{b} 2$ would not coordinate strongly to $\mathrm{LiCl}$. At $-70^{\circ} \mathrm{C}$, next to b2, a fourth species b2' is detectable in the ${ }^{1} \mathrm{H}$ experiment. At $-100^{\circ} \mathrm{C}$ species b2' can be deconvoluted in the DOSY NMR experiment (Fig. 2-27) giving a $M W_{\text {det }}$ value that fits to $\mathrm{LiCl}$ coordinated species $\mathbf{L i C l} \cdot \mathbf{D 2} \cdot \mathbf{L i C l} / \mathbf{M 1} \cdot(\mathbf{L i C l})_{2} \cdot \mathbf{M 1}(\mathbf{b 2})\left(M W_{\text {calc }}=693 \mathrm{~g} / \mathrm{mol}, M W_{\text {det }}=\right.$ $\left.641 \mathrm{~g} / \mathrm{mol}, M W_{\text {err }}=7 \%\right)$.

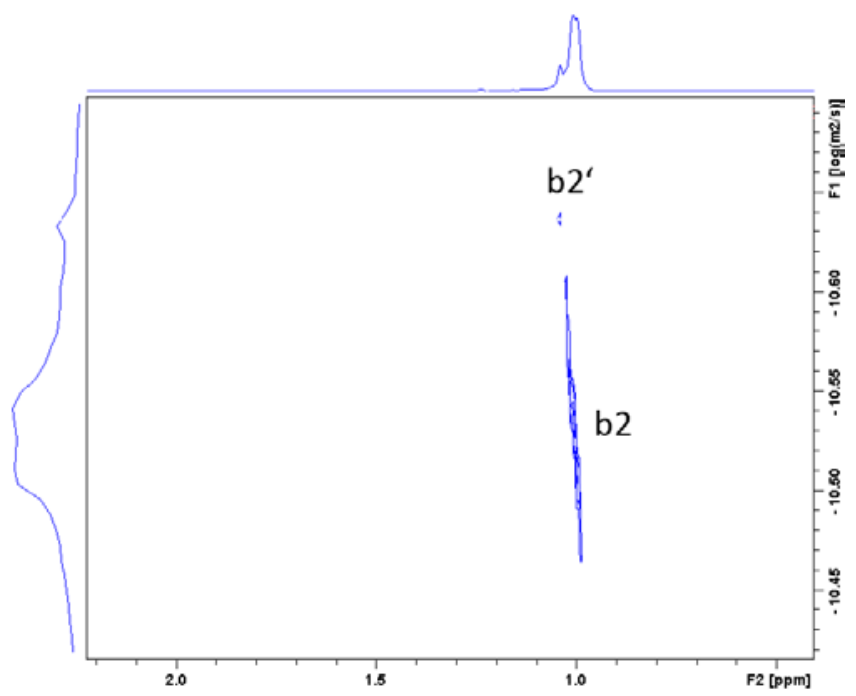

Fig. 2-27. ${ }^{1} \mathrm{H}$ DOSY spectrum of ${ }^{\mathrm{i}} \mathrm{Pr}_{2} \mathrm{NMgCl} \cdot \mathrm{LiCl} 9(0.10 \mathrm{M})$ at $-100^{\circ} \mathrm{C}$ : Region of signal $\mathbf{b 2}$ and b2'.

The ${ }^{7} \mathrm{Li}$-DOSY-ECC-MW-determination experiments give further information about the aggregation behavior of 9 . All species produce only one signal that broadens up and shifts to lower field with decreasing temperature (Fig. 2-24). Only at $0^{\circ} \mathrm{C}$ there is a small shoulder at the ${ }^{7} \mathrm{Li}$-signal, verifying that more than one $\mathrm{Li}$ species is present in solution of $\mathbf{9}$. From $25^{\circ} \mathrm{C}$ to $-40^{\circ} \mathrm{C}$ the ${ }^{7} \mathrm{Li}$-DOSY-ECC-MW-determination gives an average-, temperature dependent MW that reflects an average value produced by all three lithium aggregates $\left(M W_{\text {det }}=454-496 \mathrm{~g} / \mathrm{mol}\right)$. Below $-60^{\circ} \mathrm{C}$ the $\mathrm{MW}$ decreases significantly without further change $\left(M W_{\text {det }}=382 \mathrm{~g} / \mathrm{mol}\right.$ at $-60^{\circ} \mathrm{C}$ to $\left.-100^{\circ} \mathrm{C}\right)$. 
Chart 2-2. Most plausible aggregation modes of ${ }^{\mathrm{i}} \mathrm{Pr}_{2} \mathrm{NMgCl} \cdot \mathrm{LiCl} 9$ in $\mathrm{THF}-d_{8}$ solution. cate or $_{\text {y } A}$ category $_{\text {B }}$

(B) $=\operatorname{ipr}_{2} \mathrm{~N}$, at lower field

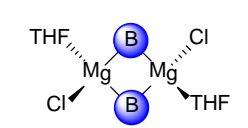

D1 $(464 \mathrm{~g} / \mathrm{mol})$

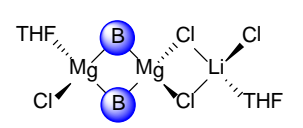

D1· LiCl $(579 \mathrm{~g} / \mathrm{mol})$

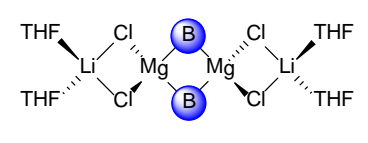

$\mathrm{LiCl} \cdot \mathrm{D} 1 \cdot \mathrm{LiCl}(693 \mathrm{~g} / \mathrm{mol})$

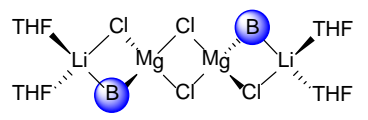

LiCl·D2*'LiCl (693 g/mol)
(B) $=\operatorname{ipr}_{2} \mathrm{~N}$, at higher field

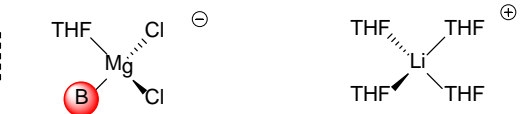

M0 (268 g/mol)

Li2 (295 g/mol)

(B) ${ }^{\mathrm{Mg}}{ }^{\mathrm{Cl}} \mathrm{THF}$

M1 (304 g/mol)

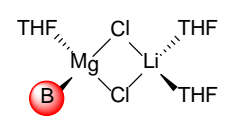

M1· $\mathrm{LiCl}(419 \mathrm{~g} / \mathrm{mol})$

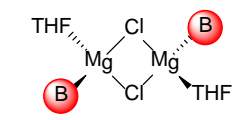

THF

D2 (464 g/mol)

Li1 (373 g/mol)

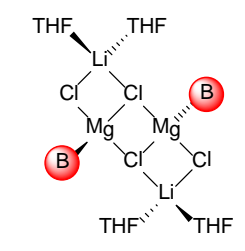

LiCl'D2'LiCl

(693 $\mathrm{g} / \mathrm{mol}$ )

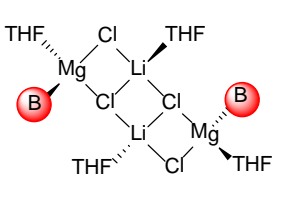

M1 $\cdot\left(\mathrm{LiCl}_{2}\right) \cdot \mathrm{M} 1$
$(693 \mathrm{~g} / \mathrm{mol})$

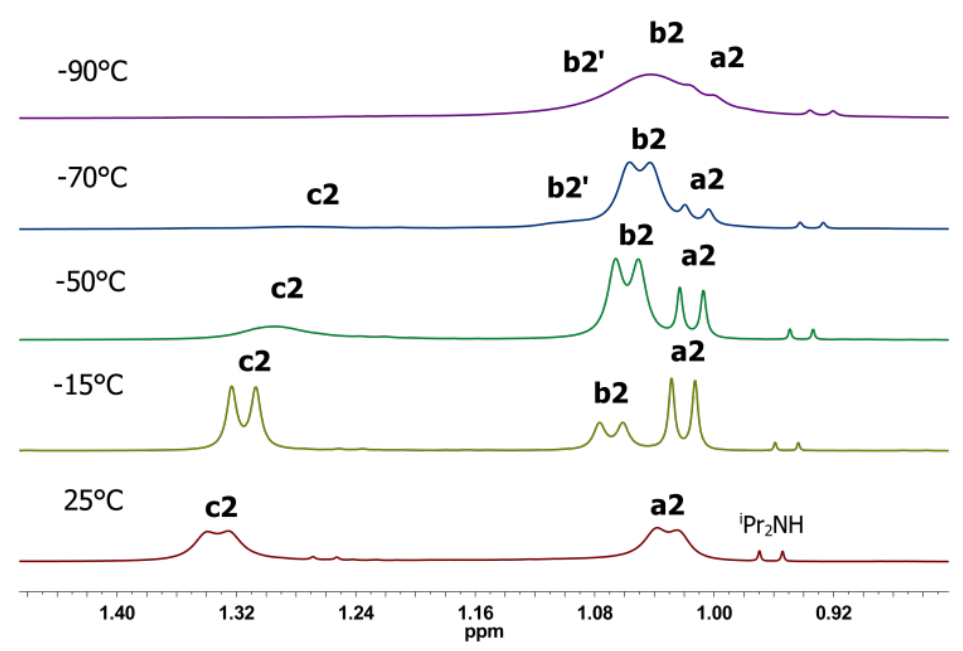

Fig. 2-26. ${ }^{1} \mathrm{H}$ superposition plot of crystalline $9\left(0.1 \mathrm{M},-\mathrm{CH}_{3}\right.$ region) re-dissolved in THF- $d_{8}$ at various temperatures. 
This can be attributed to first LiCl.D1·LiCl (c2) precipitating from solution and the concentration of M1.LiCl (a2) decreasing significantly to very small amounts and finally second to the residual lithium chloride present in solution as the well-known $\left[(\mathrm{THF})_{2} \operatorname{Li}(\mu-\mathrm{Cl})_{2} \operatorname{Li}(\mathrm{THF})_{2}\right]$-dimer Li1 $\left(M W_{\text {calc }}=373 \mathrm{~g} / \mathrm{mol}, M W_{\text {det }}=382 \mathrm{~g} / \mathrm{mol}, M W_{\text {err }}=\right.$ $-2 \%)$ that becomes the most populated Li-species at low temperatures. ${ }^{19]}$ The formation of significant amounts of Lil is also reflected in the chemical shift of the ${ }^{7} \mathrm{Li}$ nucleus. With decreasing temperature the Lil concentration increases. This is why the ${ }^{7} \mathrm{Li}$ signal moves towards the chemical shift of the LiCl dimer Lil (Fig. 2-24). Further, the signal gets very broaden. ${ }^{20}$ At low temperature the decreased solubility could be one reason for the ${ }^{7} \mathrm{Li}$ signal broadening. But additionally the formation of $\mathbf{L i C l} \cdot \mathbf{D} 2 \cdot \mathbf{L i C l} / \mathbf{M} \mathbf{1} \cdot(\mathbf{L i C l})_{2} \cdot \mathbf{M} 1$ from the reaction of $\mathrm{LiCl}(\mathbf{L i 1})$ and dimer $\mathbf{D} 2$ could have even a bigger impact on the signal broadening, since the chemical environment of lithium in $\mathbf{L i C l} \cdot \mathbf{D} 2 \cdot \mathbf{L i C l} / \mathbf{M} \mathbf{r} \cdot(\mathbf{L i C l})_{2} \cdot \mathbf{M} \mathbf{1}$ differs from that in Li1. But, how would the reaction of Lil and D2 occur? Tetrameric structures $\mathbf{L i C l} \cdot \mathbf{D} 2 \cdot \mathbf{L i C l}$ and $\mathbf{M 1} \cdot(\mathbf{L i C l})_{2} \cdot \mathbf{M 1}$ (Chart 2-2) were already proposed as products. In both structures two chlorides at the magnesium atoms show a coordination number of three. Searching the Cambridge Crystallography Database ${ }^{21}$ reveals that magnesium chlorides, with a $\mu-\mathrm{Cl}_{3}$ coordination predominantly form cubic aggregation modes in the solid state. ${ }^{[126,129]}$

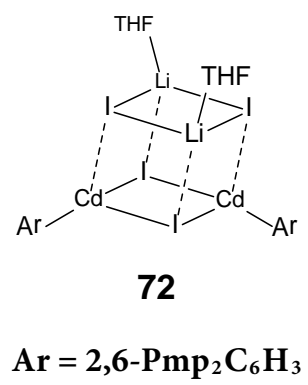

Fig. 2-28. An equimolar reaction of $\left[2,6-\mathrm{Pmp}_{2} \mathrm{C}_{6} \mathrm{H}_{3} \mathrm{Li}\right]_{2}\left(\mathrm{Pmp}=2,3,4,5,6-\mathrm{Me}_{5} \mathrm{C}_{6}\right)$ with $\mathrm{CdI}_{2}$ and crystallization from a saturated hexane solution at $-30^{\circ} \mathrm{C}$ yields crystals of cubane $72 .{ }^{[130]}$

\footnotetext{
${ }^{19}$ To prove the structure of $\mathrm{LiCl}$ in THF, DOSY measurements have been performed on anhydrous $\mathrm{LiCl}$ in THF- $d_{8}$. The ${ }^{7} \mathrm{Li}$-ECC-MW-determination confirmes the dimeric structure (Li1) of LiCl in THF solution (from $25^{\circ} \mathrm{C}$ to $-75^{\circ} \mathrm{C}$, in av.: $M W_{\text {calc }}=373 \mathrm{~g} / \mathrm{mol}, M W_{\text {det }}=381 \mathrm{~g} / \mathrm{mol}, M W_{\text {err }}=-2 \%$, see A-Table 20 in the appendix. That result stays in a very good agreement with previous work made by Reich et al. who categorized LiCl as a sturdy dimer (Li1) according to HMPA titrations: Reich, H. J.; Borst, J. P.; Dykstra, R. R.; Green, P. D. J. Am. Chem. Soc. 1993, 115, 8728-8741.

${ }^{20}$ However, a significant formation of the SSIP Li $[\mathrm{THF}]_{4}+\mathbf{L i 2}$ can be excluded $\left(M W_{\text {calc }}=295 \mathrm{~g} / \mathrm{mol}, M W_{\text {det }}=\right.$ $\left.382 \mathrm{~g} / \mathrm{mol}, M W_{\text {err }}=-29 \%\right)$.

${ }^{21}$ Based on statistics from the Cambridge Structural Database CSD, version 5.36 (Updated Nov 2014).
} 
Chart 2-2. Most plausible aggregation modes of ${ }^{\mathrm{i}} \mathrm{Pr}_{2} \mathrm{NMgCl} \cdot \mathrm{LiCl} 9$ in $\mathrm{THF}-d_{8}$ solution. cate $_{\text {gory }} \mathrm{A}$ category $_{\text {B }}$

(B) $=\operatorname{ipr}_{2} \mathrm{~N}$, at lower field

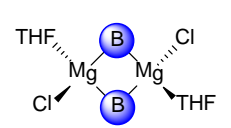

D1 $(464 \mathrm{~g} / \mathrm{mol})$

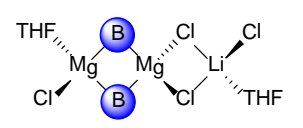

D1· LiCl $(579 \mathrm{~g} / \mathrm{mol})$

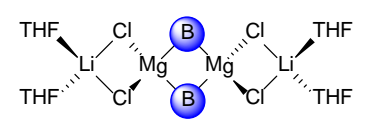

$\mathrm{LiCl} \cdot \mathrm{D} 1 \cdot \mathrm{LiCl}(693 \mathrm{~g} / \mathrm{mol})$

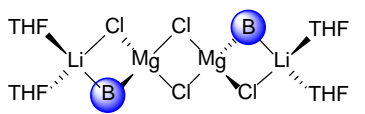

LiCl·D2*'LiCl (693 g/mol)
(B) $={ }^{i} \mathrm{Pr}_{2} \mathrm{~N}$, at higher field

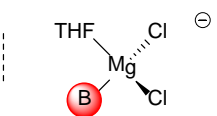

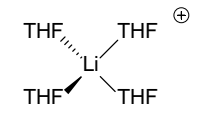

M0 (268 g/mol)

Li2 (295 g/mol)

(B) ${ }^{\mathrm{Mg}} \mathrm{THF}_{\mathrm{THF}}^{\mathrm{Cl}}$

M1 (304 g/mol)

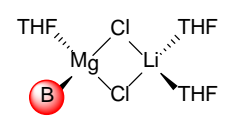

M1· LiCl $(419 \mathrm{~g} / \mathrm{mol})$

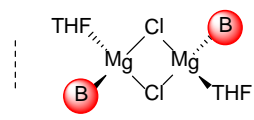

${ }_{\mathrm{THF}^{\prime}}^{\mathrm{THF}} \mathrm{Cl}_{\mathrm{CI}} \mathrm{Cl}^{\mathrm{Cl}} \mathrm{Li}_{\mathrm{THF}} \mathrm{THF}^{\mathrm{THF}}$

D2 $(464 \mathrm{~g} / \mathrm{mol})$

Li1 (373 g/mol)

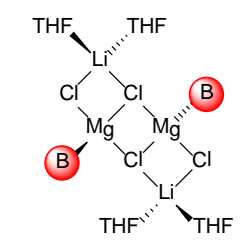

LiCl'D2' LiCl (693 $\mathrm{g} / \mathrm{mol})$

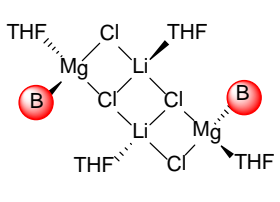

$\mathrm{M} 1 \cdot(\mathrm{LiCl})_{2} \cdot \mathrm{M} 1$

(693 $\mathrm{g} / \mathrm{mol})$

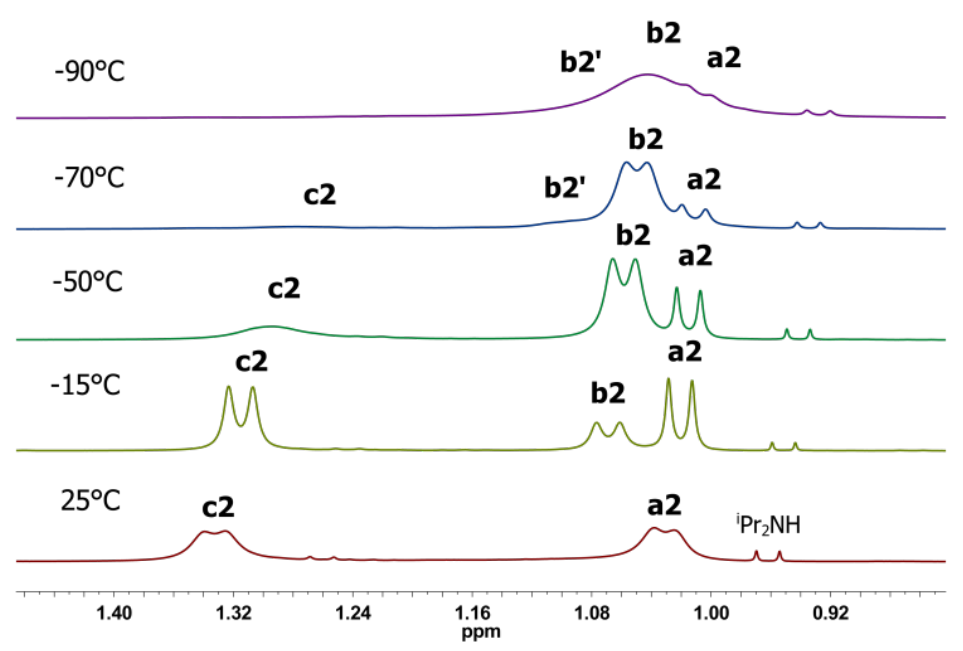

Fig. 2-26. ${ }^{1} \mathrm{H}$ superposition plot of crystalline $9\left(0.1 \mathrm{M},-\mathrm{CH}_{3}\right.$ region $)$ re-dissolved in THF- $d_{8}$ at various temperatures. 
Bickelhaupt and Solà et al. have shown that the most stable isomer of methylmagnesium chloride tetramers, $\left(\mathrm{CH}_{3} \mathrm{MgCl}\right)_{4}$, is a $\mathrm{T}_{d}$-symmetric tetranuclear cluster with a cubic $(\mathrm{MgCl})_{4}$ core and terminal $-\mathrm{CH}_{3}$ groups on the magnesium vertices. ${ }^{[131]} \mathrm{Li}$ halides are also known to build stable cubic tetramers $[\mathrm{LiX}]_{4}(\mathrm{X}=\mathrm{Cl}, \mathrm{Br}, \mathrm{I})^{[132]}$ and even heteroleptic cubanes in the solid state (see compound 72 in Fig. 2-28). ${ }^{[130,133]}$ This is why the interaction of the LiCl-dimer Li1 with dimer D2 could occur via a cubic transition state.

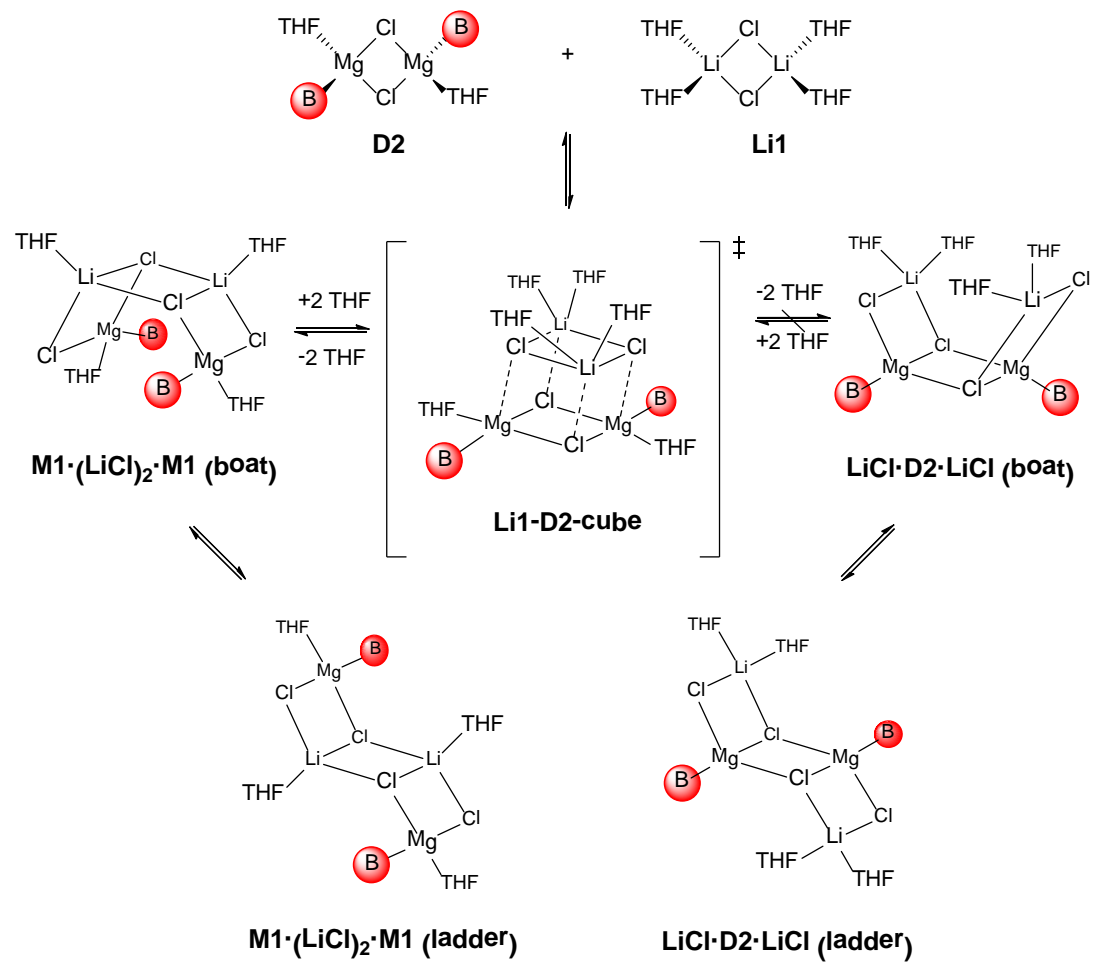

Scheme 2-4. Proposed interaction of the LiCl dimer Lil with the Hauser base dimer D2.

Since, the ring size of $[\mathrm{LiCl}]_{2}$ and $[\mathrm{MgCl}]_{2}$ differ from each other, a fast dissociation of the cubane back to the corresponding dimers would be plausible. Once the cubane is formed, it could open on one side to produce the already mentioned aggregates $\mathbf{L i C l} \cdot \mathbf{D} 2 \cdot \mathbf{L i C l}$ with a $[\mathrm{MgCl}]_{2}$ and M1.(LiCl $)_{2} \cdot \mathbf{M 1}$ with a $[\mathrm{LiCl}]_{2}$ core (Scheme $\left.2-4\right)$. To find out which of those structures is the most stable in solution further calculations were carried out. These will be discussed later in the text. Additional support for the interaction of Li1 with D2 is given by the absence of LiCl-free species M1 $\left(M W_{\text {calc }}=304 \mathrm{~g} / \mathrm{mol}\right)$ and homoleptic diorganomagnesium M2 $\left(M W_{\text {calc }}=369 \mathrm{~g} / \mathrm{mol}\right.$, see Chart 2-1, $M W_{\text {det }}($ all species $)=$ $425-661 \mathrm{~g} / \mathrm{mol}, M W_{\text {err }} \geq 28 \%$ and $M W_{\text {err }} \geq 13 \%$ ). At low temperatures M2 (Chart 2-1) is the main species in the solution of Hauser base 7 . 
Chart 2-2. Most plausible aggregation modes of ${ }^{\mathrm{i}} \mathrm{Pr}_{2} \mathrm{NMgCl} \cdot \mathrm{LiCl} 9$ in $\mathrm{THF}-d_{8}$ solution.

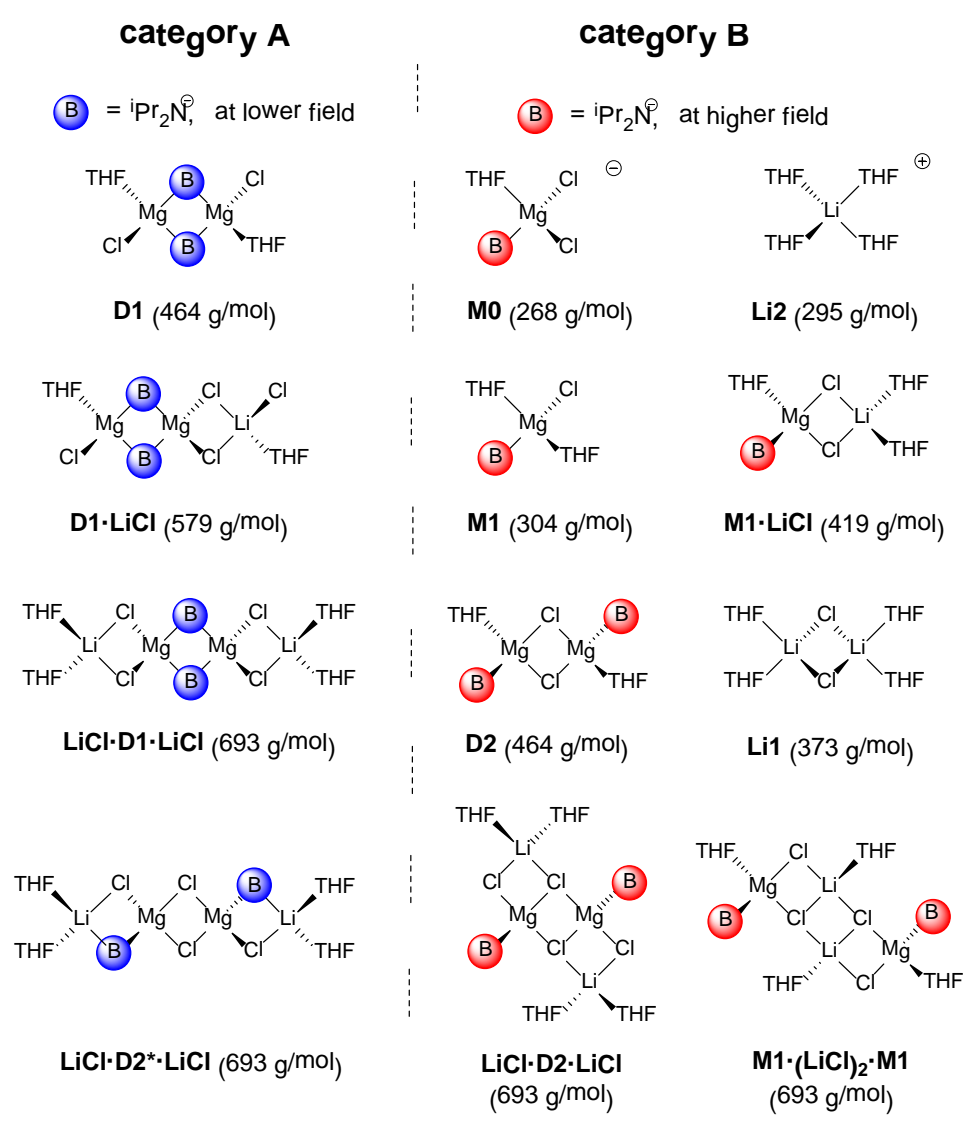


Ashby et al. proposed for the formation of M2 a dimer based mechanism (Scheme 2-5a). ${ }^{[68 a]}$ A rearrangement of dimer aII to an asymmetric dimer aIII forms two excellent leaving groups, which dissociate into the corresponding diorganomagnesium $\mathrm{R}_{2} \mathrm{Mg}$ and $\mathrm{MgCl}_{2}$ (aIV in Scheme 2-5a). In contrast, with $\mathrm{LiCl}$ as additive it is possible to rationalize several structures like bI-bVIII. Interestingly, in those structures the $\mathrm{LiCl}$ coordinated monomer bI, the LiCl-dimer bII and dimeric bIII represent always the best leaving groups (Scheme 2-5b), especially at low temperatures where monomeric $\mathrm{LiCl}$ and $\mathbf{M 1}$ are highly unfavoured.

a)

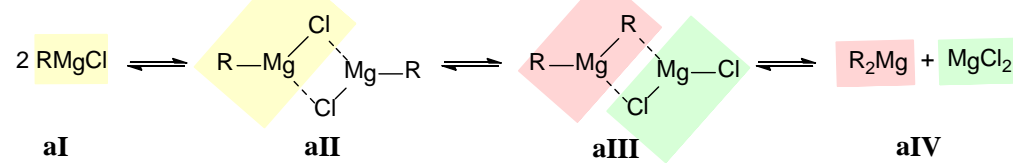

b)

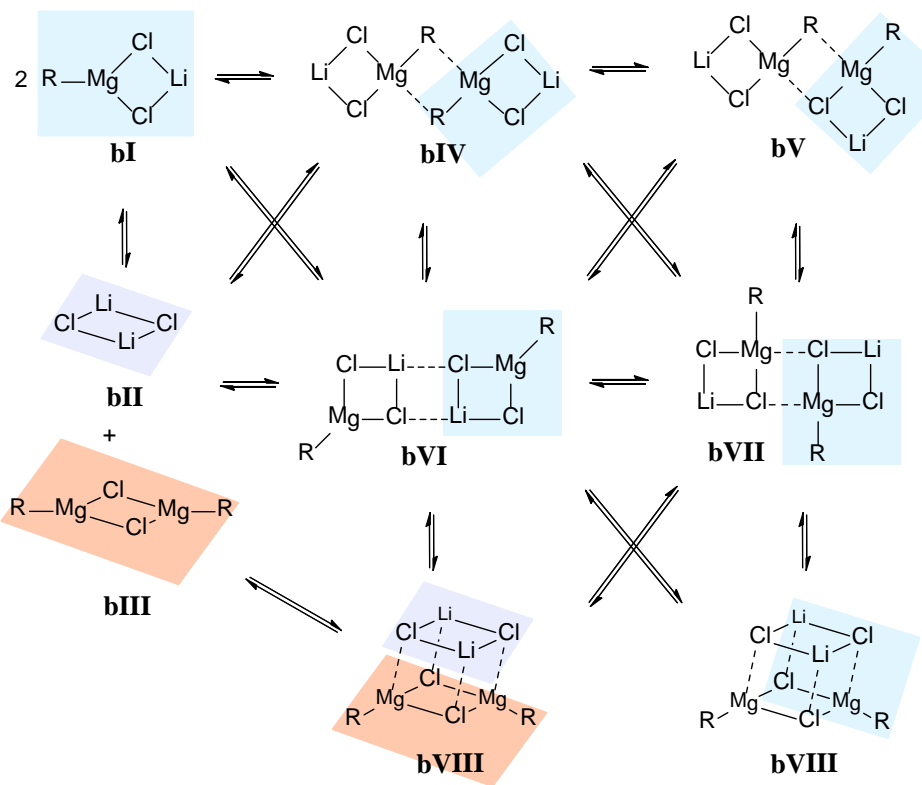

Scheme 2-5. a) Mechanism on the formation of $\mathrm{R}_{2} \mathrm{Mg}$ and $\mathrm{MgCl}_{2}$ via an asymmetric dimer aIII proposed by Ashby. b) In the presence of $\mathrm{LiCl}$ in various aggregation modes of $\mathbf{9}, \mathbf{b I}, \mathbf{b I I}$ and $\mathbf{b I I I}$ always the best leaving groups, suppressing the formation of homoleptic $\mathrm{R}_{2} \mathrm{Mg}$ and $\mathrm{MgCl}_{2}$ aIV; any solvation omitted for clarity. 
Chart 2-2. Most plausible aggregation modes of ${ }^{\mathrm{i}} \mathrm{Pr}_{2} \mathrm{NMgCl} \cdot \mathrm{LiCl} 9$ in $\mathrm{THF}-d_{8}$ solution.

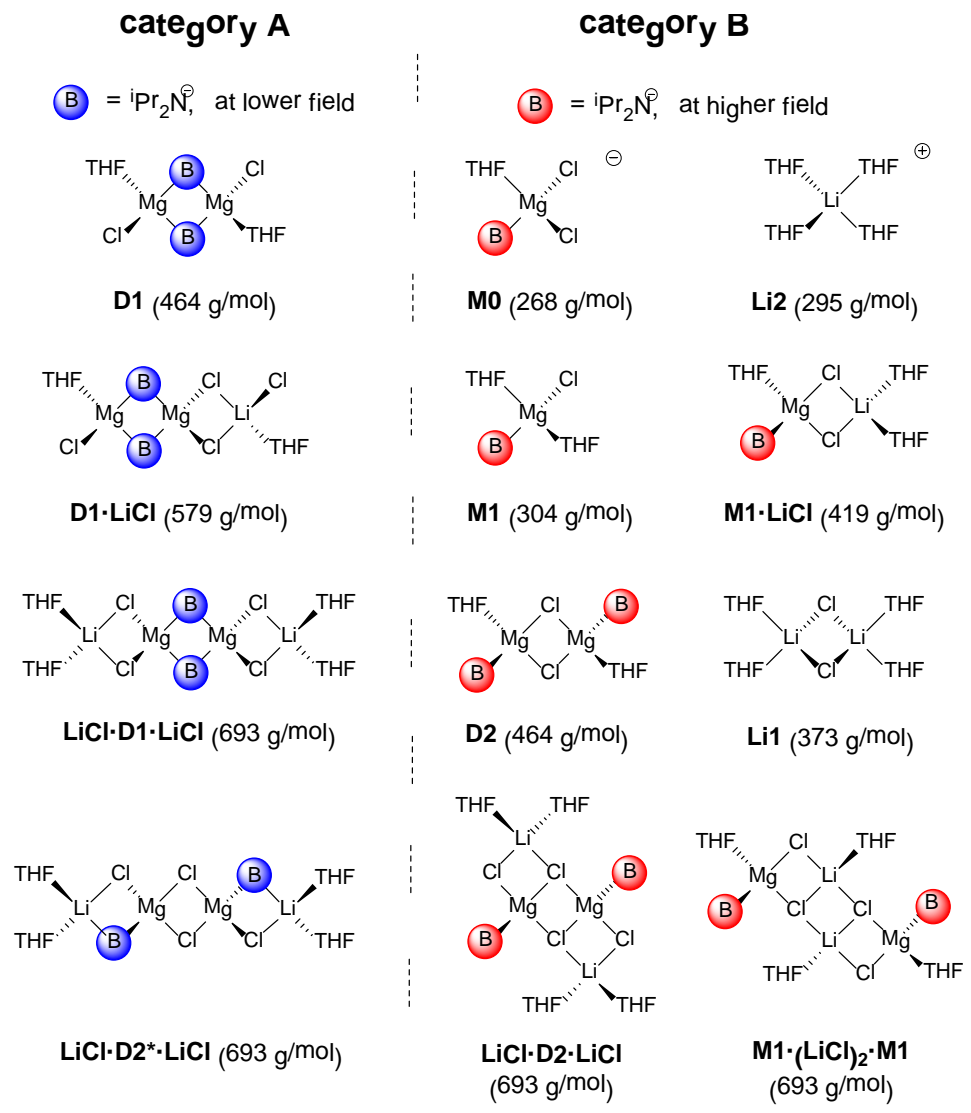


Further, the cleavage of $\mathrm{MgCl}_{2}$ or $\mathrm{R}_{2} \mathrm{Mg}$ would be accompanied by a release of solvent separated ions that would be very unfavourable since no solvent separated species were observable in solution of Hauser base $\mathbf{1}$ and 2. ${ }^{22}$ If the $\mathrm{LiCl}$ dimer Lil (bII) would not communicate with dimer D2 (bIII) than the formation of homoleptic M2 (aIV) should be observable which is not the case. ${ }^{[71]}$ Without $\mathrm{LiCl}$ the dimer $\mathbf{D 2}$ is only stable above $-50^{\circ} \mathrm{C}$. But with $\mathrm{LiCl}$ it is the main species below $-50^{\circ} \mathrm{C}$ in Hauser base 2 most probably because of its interaction with the $\mathrm{LiCl}$ dimer Li1. This contrasting behaviour could be reflected in the different aggregation modes of $\mathrm{LiCl}$ and $\mathrm{MgCl}_{2}$ in $\mathrm{THF}$ solution. The former is predominately dimeric, enabling a sufficient overlap of the $[\mathrm{LiCl}]_{2}$ ring with dimer $\mathbf{D 2}$, while $\mathrm{MgCl}_{2}$ is predominately monomeric. ${ }^{[124 b]}$ This is why $\mathrm{MgCl}_{2}$ stabilize more efficiently in a terminal coordination mode like e.g in $\mathbf{M} \mathbf{1}\left(\boldsymbol{\mu}-\mathbf{C l}_{3}\right) \mathbf{M g C l}_{2}$ and $\mathbf{M g C l}_{2} \cdot \mathbf{D} 1(\mathbf{D} 2) \cdot \mathbf{M g C l}_{2}$. To address some of the open questions regarding the interaction and aggregation of Li1 and D2 in THF solution, we carried out further calculations on the complexes. We started by computing the relative free energies for $\mathbf{M 1} \cdot(\mathbf{L i C l})_{2} \cdot \mathbf{M} 1$ and $\mathbf{L i C l} \cdot \mathbf{D} 2 \cdot \mathbf{L i C l}$ (depicted in Scheme 2-4). The results show that the ladder and boat configurations, both for the $\mathbf{M 1} \cdot(\mathbf{L i C l})_{2} \cdot \mathbf{M} 1$ and the $\mathbf{L i C l} \cdot \mathbf{D} 2 \cdot \mathbf{L i C l}$ isomers are very close in energy (A-Table 25 in the appendix), with differences below $3 \mathrm{~kJ} / \mathrm{mol}$ (at $25^{\circ} \mathrm{C}$ ). This is definitely within the uncertainty of the method and would indicate no particular conformational preference. However, the structures $\mathbf{M 1} \cdot(\mathbf{L i C l})_{2} \cdot \mathbf{M} \mathbf{1}$ with an inner $[\mathrm{LiCl}]_{2}$ ring are identified as the by $42.2 \mathrm{~kJ} / \mathrm{mol}$ in free energy most stable species.

$$
\begin{aligned}
& \mathrm{D} 2+\mathrm{Li1} \quad \mathrm{M1} \cdot(\mathrm{LiCl})_{2} \cdot \mathrm{Ml}+2 \mathrm{THF} \\
& (-3.3 \text { to }-14.3 \mathrm{~kJ} / \mathrm{mol}) \\
& 2 \mathrm{M} 1 \cdot \mathbf{L i C l} \rightleftharpoons \mathbf{M 1} \cdot(\mathbf{L i C l})_{2} \cdot \mathbf{M} 1+2 \mathrm{THF} \\
& (17.3 \text { to } 3.9 \mathrm{~kJ} / \mathrm{mol}) \\
& \mathrm{D} 2+\mathrm{Li1} \quad \Longrightarrow \quad \text { LiCl.D1·LiCl }+2 \mathrm{THF} \\
& (-58.5 \text { to }-68.1 \mathrm{~kJ} / \mathrm{mol}) \\
& 2 \mathrm{M1} \cdot \mathrm{LiCl} \quad \Longrightarrow \quad \text { LiCl.D1·LiCl }+2 \mathrm{THF} \\
& (-37.9 \text { to }-49.9 \mathrm{~kJ} / \mathrm{mol})
\end{aligned}
$$

\footnotetext{
${ }^{22}$ Grignard reagents are also known to be very weak electrolytes, see Seyferth, Organometallics, 2009, 28, 1598-1605.
} 
Chart 2-2. Most plausible aggregation modes of ${ }^{\mathrm{i}} \mathrm{Pr}_{2} \mathrm{NMgCl} \cdot \mathrm{LiCl} 9$ in $\mathrm{THF}-d_{8}$ solution.

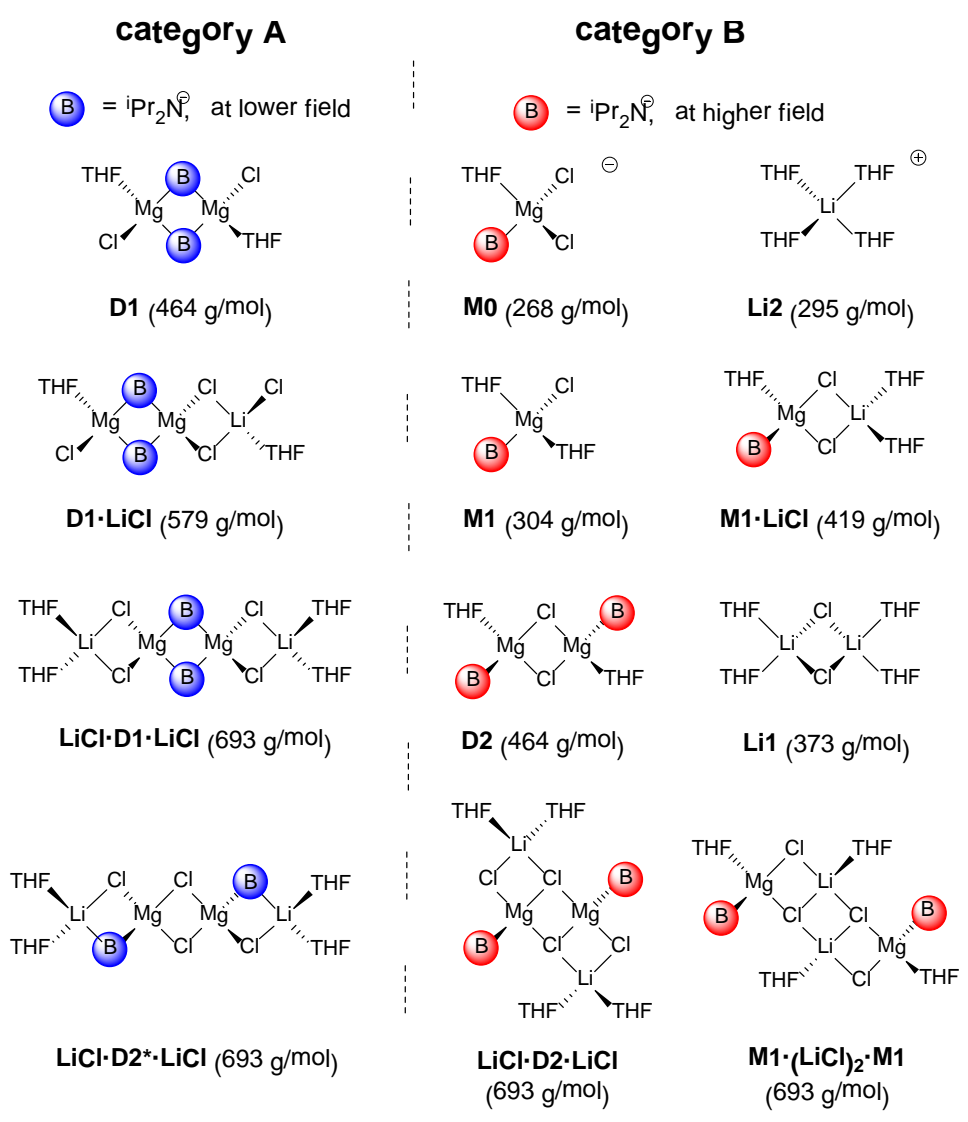


The next question is whether the formation of $\mathbf{M 1} \cdot(\mathbf{L i C l})_{2} \cdot \mathbf{M 1}$ is favoured in solution as well. This complex can be formed e.g. by the reaction of D2 with Li1 (III) or by dimerization of two M1· $\mathbf{L i C l}$ molecules (IV). The free energies for the mixed Li-Mg dimer formation were computed in a range from $-90^{\circ} \mathrm{C}$ to $+25^{\circ} \mathrm{C}$, revealing that the dimerization process of $\mathbf{D 2}$ and $\mathbf{L i 1}$ (III) to give $\mathbf{M 1} \cdot(\mathbf{L i C l})_{2} \cdot \mathbf{M 1}$ at low temperature is slightly exergonic ( -3.3 to $-14.3 \mathrm{~kJ} / \mathrm{mol}$, from lower to higher temperature, A-Table 27 in the appendix). Species M1·( $\mathbf{L i C l})_{2} \cdot \mathbf{M 1}$ (b') could, as such, be formed in the solution of Turbo-Hauser base 9. In contrast, the dimerization of two $\mathbf{M 1} \cdot \mathbf{L i C l}$ molecules (IV) is unfavoured due to an endergonic reaction (17.3 to $3.9 \mathrm{~kJ} / \mathrm{mol}$, A-Table 29 in the appendix). Obviously such values have to be considered with certain reservations, since the molarity is changing (formally, two THF molecules are released into solution during the process, so the formation of 3 molecules from 2 starting products is present). The quantum chemical result is, therefore, strongly influenced by the translation entropy. In the gas phase, the latter can be computed from the harmonic vibrational partition function. This does not apply to the case in solution, since solvated molecules are not free to move and possess lower translational entropy than in the gas. The theoretical calculations already include a correction as suggested by Ardura et al. on the basis of a cell model for the change in translational degrees of freedom. ${ }^{[134]}$ However, this little energy gain for the formation of M1 $(\mathbf{L i C l})_{2} \cdot \mathbf{M 1}$ stays in good agreement with the presented NMR experiments, showing that D2 and Lil interact and communicate with each other but still the equilibrium is highly on the side of free $\mathbf{D} 2$ and Li1. In tune with the crystal structure of $[\mathbf{9} \cdot \mathrm{THF}]_{2}$ the highest energy gain was identified for the formation of LiCl-D1·LiCl (V and VI). Again the reaction of $\mathbf{D} 2$ and $\mathbf{L i 1}$ is preferred over the dimerization of two $\mathbf{M 1} \cdot \mathbf{L i C l}$ molecules by $19.4 \mathrm{~kJ} / \mathrm{mol} .{ }^{23}$ Interestingly, the solubility limit of $\mathbf{L i C l} \cdot \mathbf{D} \mathbf{1} \cdot \mathbf{L i C l}$ is reached at approximately $-70^{\circ} \mathrm{C}$ providing small amounts of $\mathbf{M 1} \cdot(\mathbf{L i C l})_{2} \cdot \mathbf{M 1}$ that is thermodynamically less stable.

One additional open question is the existence of cubic intermediates, through which the larger mixed $\mathrm{Li}-\mathrm{Mg}$ aggregates could be formed. Since there is no experimental data available on such transient species, one can only postulate about different coordination motifs. Four possible cubane structures Li1·D2-cube(A-D) are proposed (Fig. 2-29), with two available coordination sites at each $\mathrm{Li}$ and additional two at each $\mathrm{Mg}$ site. The calculations clearly identify complexes $\mathbf{B}$ and $\mathbf{C}$ as the most unstable, given the low coordination number of the $\mathrm{Mg}$ sites.

\footnotetext{
${ }^{23}$ Again with a correction to the translational entropy.
} 

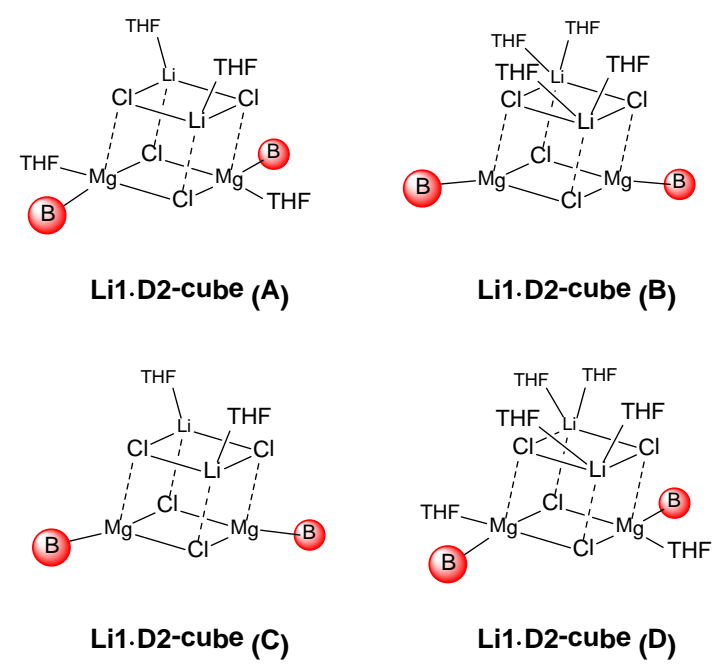

Fig. 2-29. Overview of possible cube structures of Turbo-Hauser base 9.

Chart 2-2. Most plausible aggregation modes of ${ }^{\mathrm{i}} \mathrm{Pr}_{2} \mathrm{NMgCl} \cdot \mathrm{LiCl} 9$ in $\mathrm{THF}-\boldsymbol{d}_{8}$ solution.

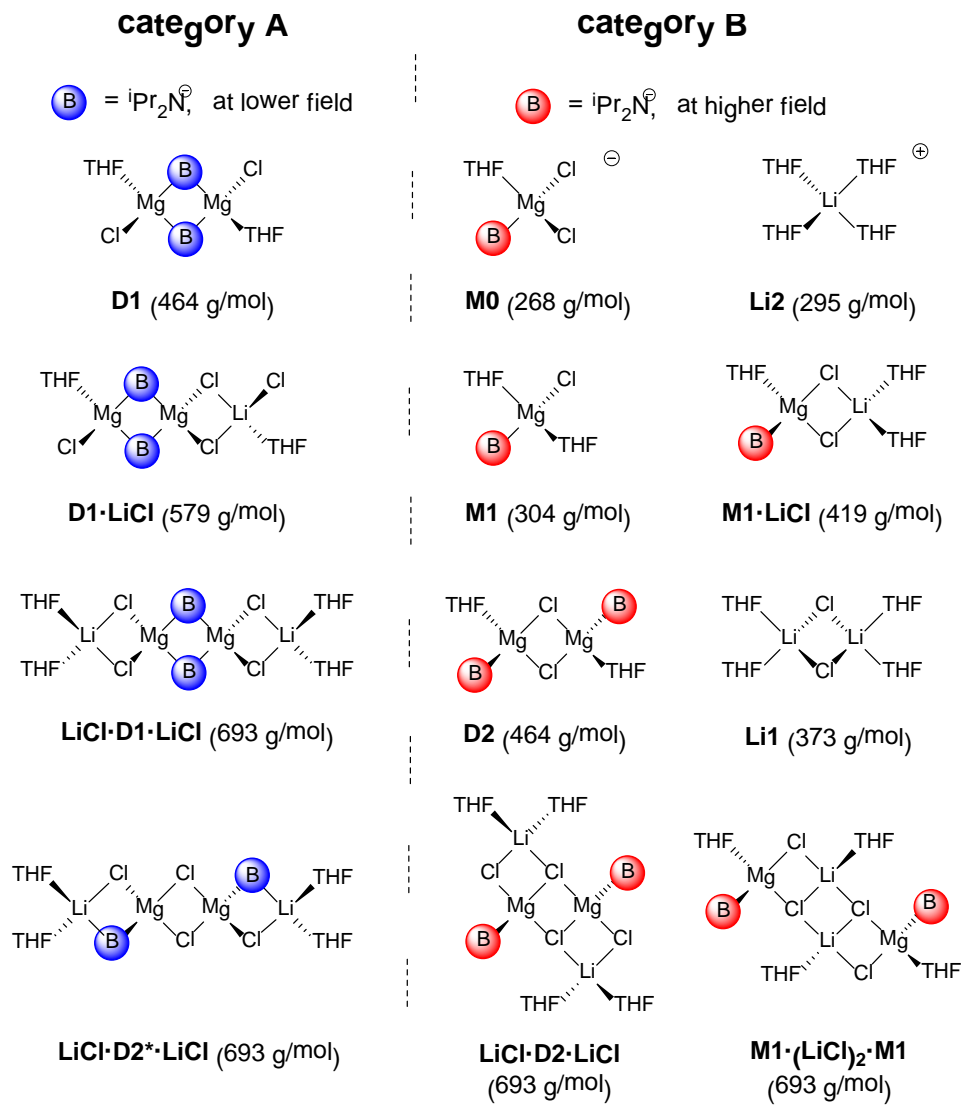


A direct comparison can be in fact established between Li1·D2-cube(A) and Li1·D2cube(B), with $\mathbf{A}$ being favoured over $\mathbf{B}$ in the considered temperature range by about $10 \mathrm{~kJ} / \mathrm{mol}$ (A-Table 24 in the appendix). For the preferred cubane structures $\mathbf{A}$ and $\mathbf{D}$ one might consider the associated equilibria given by the general equations:

$$
\begin{aligned}
& \mathrm{D} 2+\text { Li1 } \rightleftharpoons \quad \text { LiCl.D2-cube }+n \mathrm{THF} \\
& 2 \mathrm{M} 1 \cdot \mathbf{L i C l} \quad \Longrightarrow \quad \text { LiCl} \cdot \mathbf{D} 2-\text { cube }+n \mathrm{THF}
\end{aligned}
$$

with $n=0$ for $\mathbf{D}$ and 2 for $\mathbf{A}$. As mentioned before, the change in molarity is an obstacle in computing the free energies. By considering two equilibria simultaneously, where in one case the molarity change entropically promotes the products (A) and in the other the reactants (D). The computed free energies for dimerization according to equilibrium (VII) are given in Fig. 2-30. The dimerization of two $\mathbf{M 1} \cdot \mathbf{L i C l}$ molecules to form Li1·D2-cube(AD) (VIII) is in average $19.4 \mathrm{~kJ} / \mathrm{mol}$ higher in energy (A-Table 26 and A-Table 28 in the appendix).

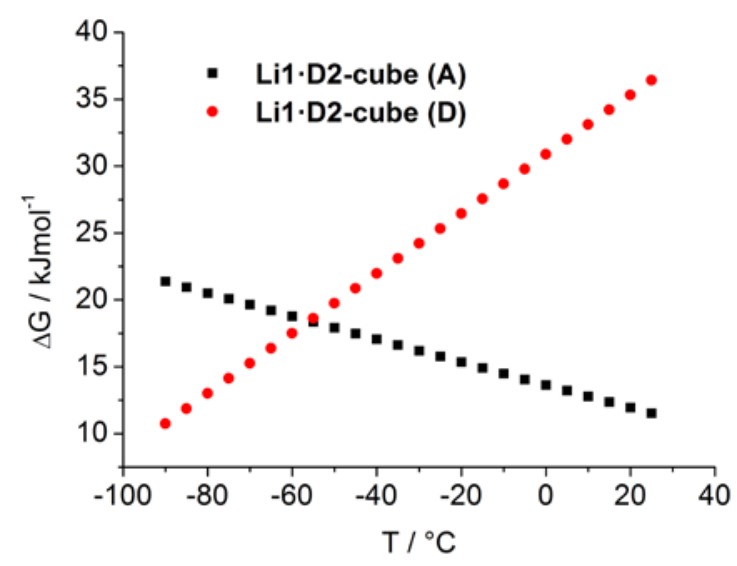

Fig. 2-30. Computed free energies for dimerization of D2 + Lil to give LiCl·D2-cube (A) and LiCl-D2cube(D).

As expected, the formation of complex $\mathbf{D}$ would be most favourable at lower temperatures. The crossing point is at about $-55^{\circ} \mathrm{C}$. Although the equilibrium is always shifted towards the reactants, the differences can be relatively small, as low as $10.7 \mathrm{~kJ} / \mathrm{mol}$, such that the cubic intermediate should be accessible, even if not stable relative to the metal dimer complexes D2 and Li1. 
Chart 2-2. Most plausible aggregation modes of ${ }^{\mathrm{i}} \mathrm{Pr}_{2} \mathrm{NMgCl} \cdot \mathrm{LiCl} 9$ in $\mathrm{THF}-d_{8}$ solution.

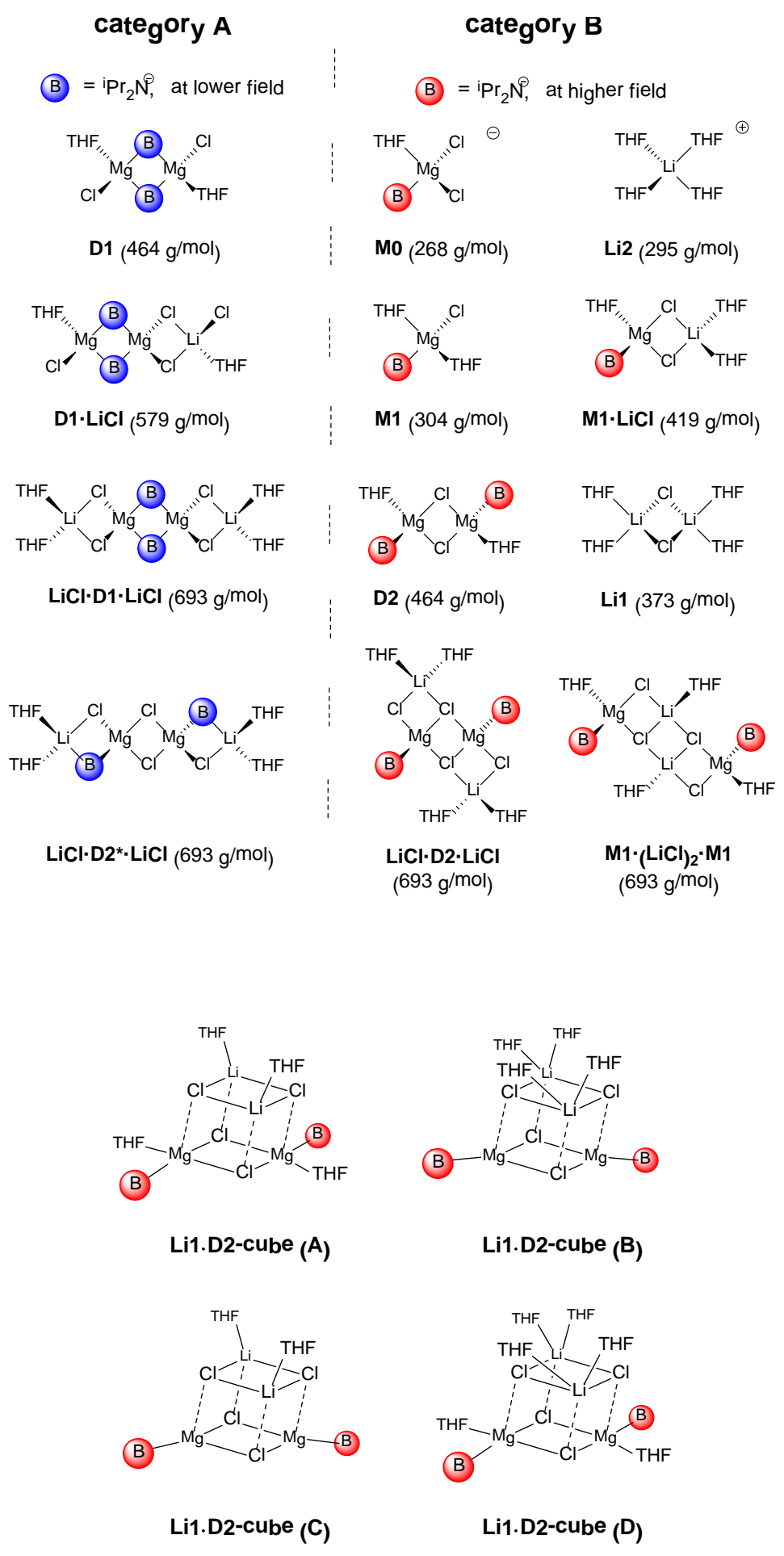

Fig. 2-29. Overview of possible cube structures of Turbo-Hauser base 9. 
The presented results show that the formation of small amounts of $\mathbf{M 1} \cdot(\mathbf{L i C l})_{2} \cdot \mathbf{M} 1$ (Scheme 2-6) is thermodynamically possible. Most probably, this complex is formed by dimerization of D2 with Li1 via a cubic intermediate, presumably Li1·D2-cube(D) at temperatures below $-55^{\circ} \mathrm{C}$. In addition, the $\mathrm{NMR}$ investigations show also that ${ }^{i} \operatorname{Pr}_{2} \mathrm{NMgCl} \cdot \mathrm{LiCl} 9$ does not produce SSIPs in detectable concentrations. ${ }^{24}$ Further, the proposal that the impact of $\mathrm{LiCl}$ on the higher reactivity of Turbo bases rests on the deaggregation of $\mathrm{RMgX}$ oligomers to monomers has to be revised. Most reactions of $\mathrm{RMgX}$ reagents proceed in THF solution and it is shown for long time that alkyl and aryl Grignard reagents are monomeric in THF solution. ${ }^{[66]}$ The results show that same seems to be true for Hauser base 7.

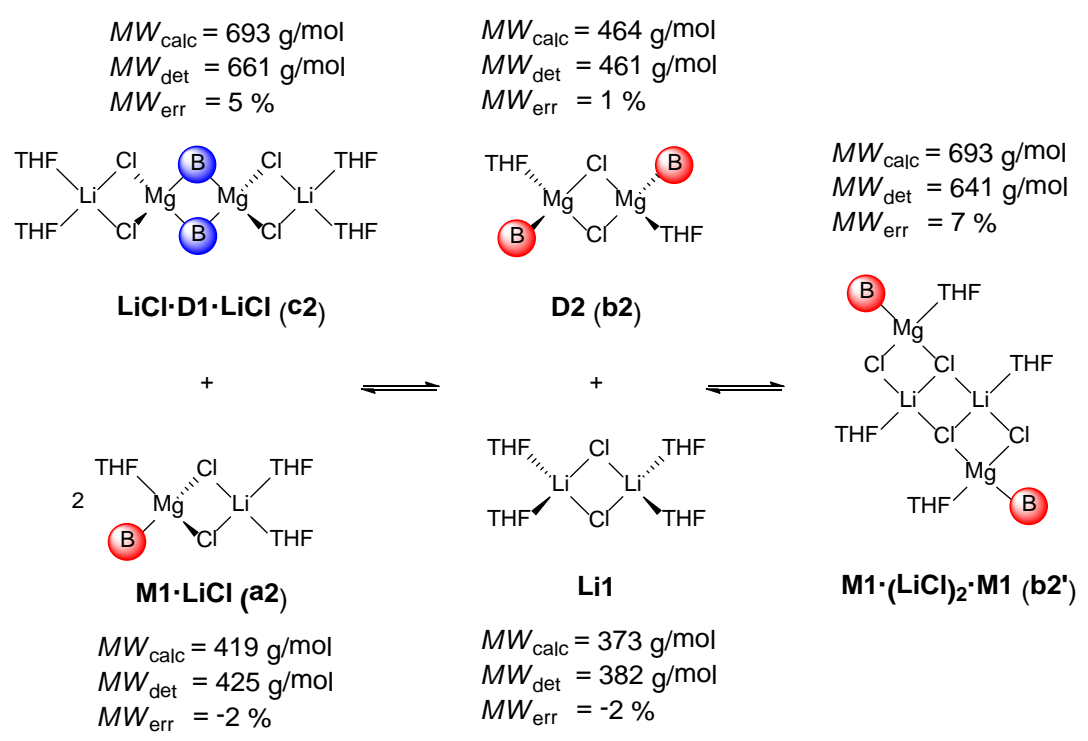

Scheme 2-6. Schematic representation of the solution structure of 9 in THF- $d_{8}$ solution. The MWs were derived from ${ }^{1} \mathrm{H}$ - and ${ }^{7} \mathrm{Li}$-DOSY-ECC-MW-determinations. At RT the equilibrium of $\mathbf{9}$ is highly located on the left side. At low temperature it moves significantly to the middle side.

Moreover, the reason for the lower reactivity of LiCl- free Hauser bases should be reflected in the Schlenk-equilibrium. At low temperatures the equilibrium in THF solution is mostly shifted to the side of homoleptic diamidomagnesium $\mathrm{R}_{2} \mathrm{Mg}$ where the amide ligands are highly sterically hindered in comparison with the heteroleptic $\mathrm{RMgCl}$ monomers and $\mathrm{MgCl}_{2}$ co-coordinated species. These compounds that represent the most reactive species in a Hauser base solution are only present at low concentrations. This explains why it is necessary to use a large excess of Hauser bases (2-12 fold) to achieve high conversions in synthesis. ${ }^{[23]}$ The big advantage of $\mathrm{LiCl}$ is the ability to shift the Schlenk-equilibrium from

\footnotetext{
${ }^{24}$ Additionally, a significant formation of the SSIP Li[THF $]_{4}+\mathbf{L i} 2$ can be excluded $\left(M W_{\text {calc }}=295 \mathrm{~g} / \mathrm{mol}\right.$, $\left.M W_{\text {det }}=382 \mathrm{~g} / \mathrm{mol}, M W_{\text {err }}=-29 \%\right)$
} 
the homoleptic to the heteroleptic side, especially at low temperatures. The high concentration of bimetallic complexes (like monomeric $\mathbf{M 1} \cdot \mathbf{L i C l}(\mathbf{a} \mathbf{1})$ as well as dimeric LiCl·D1·LiCl (c2), D2 (b2) and M1·(LiCl) ${ }_{2} \cdot \mathbf{M 1}(\mathbf{b 2}$ ') should provide the most influence on the reactivity, chemoselectivity and complex induced proximity effects (CIPE) ${ }^{[21]}$ of TurboHauser bases. It is possible that this concept could also be applied to Turbo-Grignard reagents.

\subsubsection{Structure of Turbo-Hauser Base TMPMgCl- $\mathrm{LiCl}$ in THF solution $^{25}$}

Chart 2-3. The most plausible solution structures of 9 and 10 in THF-d d $_{8}$ solution

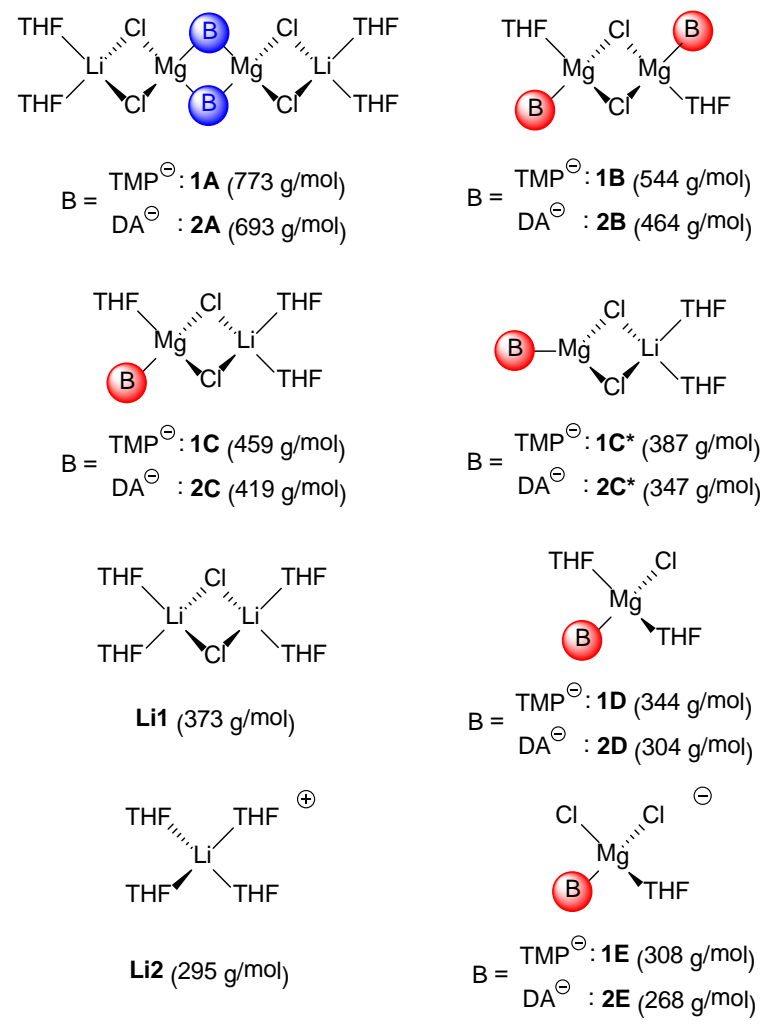

Turbo-Hauser bases ${ }^{i} \mathrm{Pr}_{2} \mathrm{NMgCl} \cdot \mathrm{LiCl} 9$ and TMPMgCl$\cdot \mathrm{LiCl} 10$ show a different reactivity and regioselectivity. ${ }^{[23]}$ To understand this property and to be able to deduce informative structure-reactivity relationships it is extraordinary important to explore the solution structure of 10. García-Álvarez and Mulvey et al. analyzed crystals of 1C in THF- $d_{8}$ solution $^{[28]}$ by diffusion ordered spectroscopy (DOSY) and the diffusion coefficient formula weight (D-FW) analysis that was pioneered by $L i$ and Williard et al. ${ }^{[77,104 a]}$ This method is

\footnotetext{
${ }^{25}$ A revised version of my manuscript: R. Neufeld, D. Stalke, Chem. Eur. J. 2016, submitted.
} 
based on internal calibration curves (ICC), where many internal standards which may interfere with the reactive metal complexes are required. Because of peak overlap problems the authors had to use inappropriate internal standards, ${ }^{26}$ so the molecular weight (MW) determination was prone to a relatively high error of approximately $\pm 30 \%$. Consequently García-Álvarez and Mulvey et al. stated that they were not able to "clearly establish the exact nature of the solution species". ${ }^{[28]}$ Predominantly it could not be established whether or not lithium chloride is co-coordinated to the magnesium amide. Anyhow it was concluded that a SSIP situation appeared to be the most feasible. In the following section it will be proved that $\mathrm{LiCl}$ does indeed coordinate to Turbo-Hauser Base $\mathbf{1 0}$.

Primarily it seems advisable to a priori rationalize which species are feasible to be present in the solution of $\mathbf{1 0}$. The most obvious question to be addressed in s-block organometallics of course is the amount of coordinating THF molecules to be present in the solution structure of 10. Even from a vast number of solid state structures it is known that polar solvents like THF are necessary to coordinate such highly ionic compounds. A re-dissolved crystal of $\mathbf{1 0}$ in net THF could on the one hand retain its solid state structure (1C) or even aggregate further to combine to dimers $(\mathbf{1 A}, \mathbf{1 B})$ as well as dissociate to a number of smaller molecules $\left(\mathbf{1 C}^{\star}-\mathbf{1 E}\right.$, Chart 2-3). $\mathrm{LiCl}$ can either coordinate to the magnesium amide or dissociate as a well-known $\left[(\mathrm{THF})_{2} \mathrm{Li}(\mu-\mathrm{Cl})_{2} \mathrm{Li}(\mathrm{THF})_{2}\right] \operatorname{dimer}^{[127]} \mathbf{L i 1}$. A solvent separated ion pair (SSIP) $\mathrm{Li}(\mathrm{THF})_{4}{ }^{+} \mathbf{L i} 2$ would promote the formation of an atecomplex 1E where two chloride ions coordinate a single magnesium cation. From the crystal structure of $\mathbf{1 0}$ it is known that the lithium cation is located at an average distance of $4.68 \AA$ to the closest $\mathrm{CH}_{3}$-protons of the TMP ligand. ${ }^{[72]}$ This relatively close distance should be detectable in a ${ }^{1} \mathrm{H}-{ }^{7} \mathrm{Li}-\mathrm{HOESY}$ experiment when the structure is retained in solution. ${ }^{[13 b]}$ Indeed the ${ }^{1} \mathrm{H}-{ }^{7} \mathrm{Li}-\mathrm{HOESY}$-spectra at all temperatures display a cross peak between lithium and the $\mathrm{CH}_{3}$ groups confirming the lithium coordination to the magnesium amide (Fig. 2-31). Similar results where observed for Hauser base 9.

\footnotetext{
${ }^{26}$ The error of the DOSY-MWanalysis depends highly on the shape of the used references as well on that of the analyte (see chapter 2.1.4). Hauser bases have an ellipsoidal shape. Therefore ellipsoid references should be used for an accurate MW-analysis. However, García-Álvarez et al. used references with different shapes (spherical, ellipsoidal and flat discs) that dramatically decreased the accuracy of the MW-determination.
} 


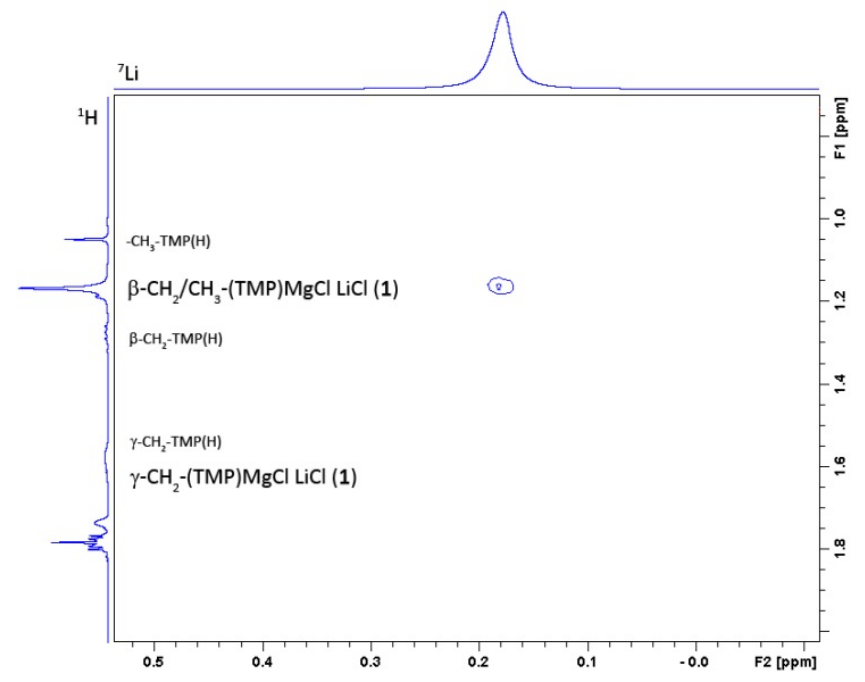

Fig. 2-31. ${ }^{1} \mathrm{H}-{ }^{7} \mathrm{Li}-\mathrm{HOESY}$ at $-50^{\circ} \mathrm{C}$ of crystalloid 10 re-dissolved in THF- $d_{8}(20 \mathrm{~mm})$ at various temperatures.

Chart 2-3. The most plausible solution structures of 9 and 10 in THF-d Th $_{8}$ solution

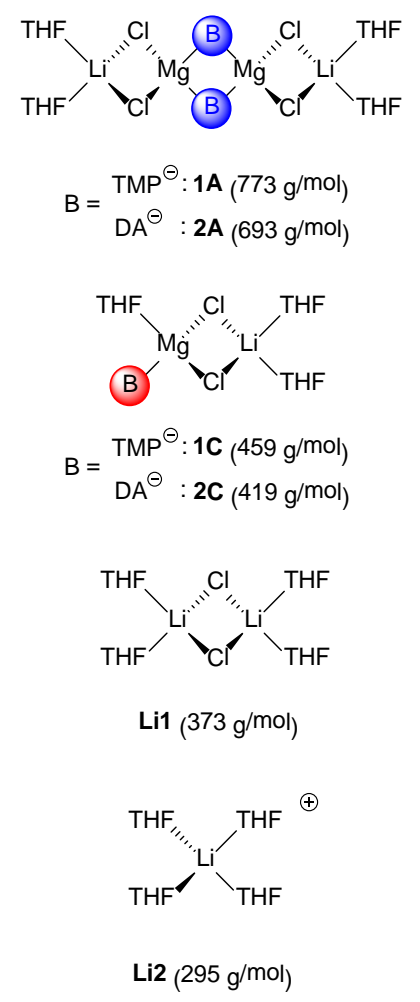

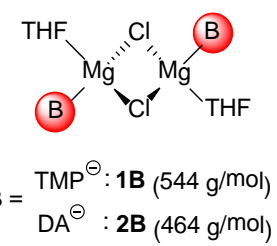

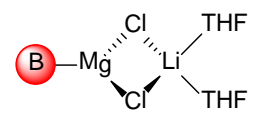

$B=\begin{aligned} & \operatorname{TMP}^{\ominus}: 1 C^{*}(387 \mathrm{~g} / \mathrm{mol}) \\ & \operatorname{DA}^{\ominus}: 2 C^{*}(347 \mathrm{~g} / \mathrm{mol})\end{aligned}$

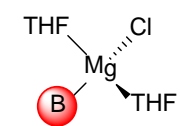

$B=\begin{aligned} & \operatorname{TMP}^{\ominus}: 1 D(344 \mathrm{~g} / \mathrm{mol}) \\ & D^{\ominus}: 2 D(304 \mathrm{~g} / \mathrm{mol})\end{aligned}$

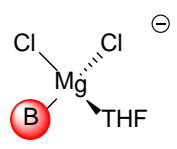

$B=\begin{aligned} & \operatorname{TMP}^{\ominus}: 1 E(308 \mathrm{~g} / \mathrm{mol}) \\ & \operatorname{DA}^{\ominus}: 2 E(268 \mathrm{~g} / \mathrm{mol})\end{aligned}$ 


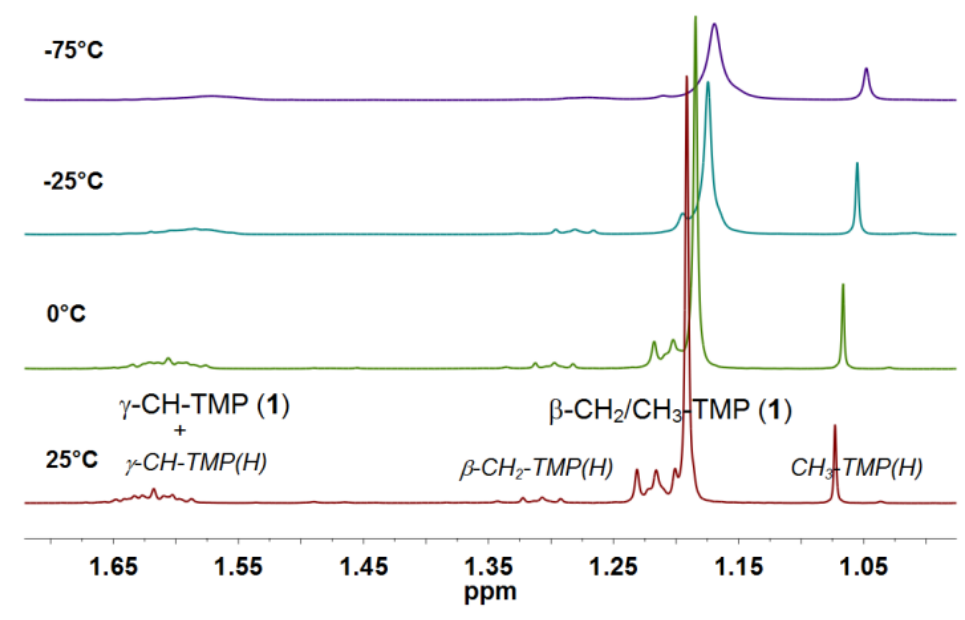

Fig. 2-32. Superposition of ${ }^{1} \mathrm{H}$ NMR spectra of crystalloid 10 re-dissolved in THF- $d_{8}$ at various temperatures.

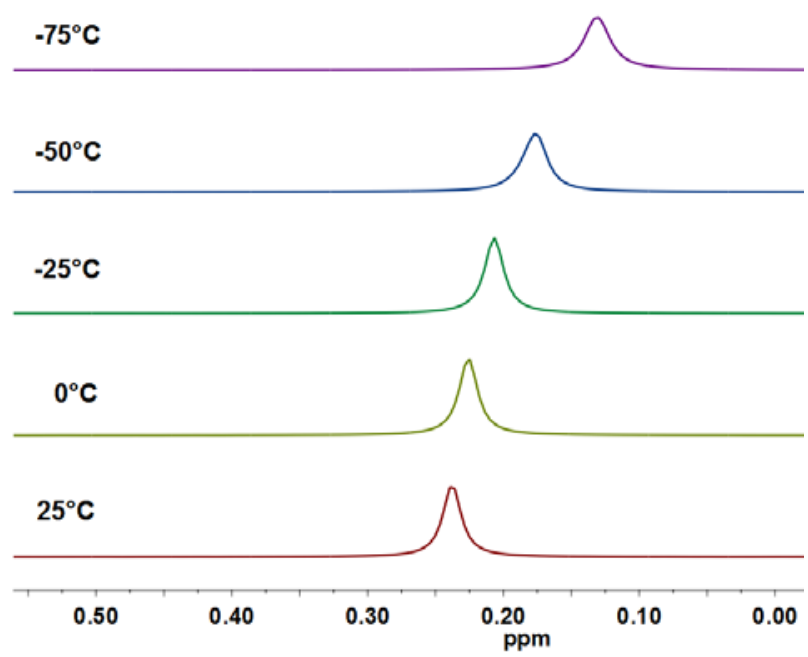

Fig. 2-33. Superposition of ${ }^{7} \mathrm{Li}$ NMR spectra of crystalloid 10 re-dissolved in THF- $d_{8}(20 \mathrm{~mm})$ at various temperatures.

For NMR spectroscopic measurements diluted solutions of $10(20 \mathrm{~mm})$ where used by dissolving the crystals in THF- $d_{8}$. The ${ }^{1} \mathrm{H}$ NMR spectra of 10 show at all temperatures $\left(25^{\circ} \mathrm{C}\right.$ to $\left.-75^{\circ} \mathrm{C}\right)$ only one single type of TMP ligand $\left(\delta=1.19 / 1.22 / 1.62 \mathrm{ppm}\right.$ for $\mathrm{CH}_{3} / \beta$ $\mathrm{CH}_{2} / \gamma$-CH, Fig. 2-32) while the ${ }^{i} \operatorname{Pr}$-Turbo-Hauser base 9 displays several oligomers. ${ }^{27}$ The ${ }^{7} \mathrm{Li}$ NMR spectra show one singlet at about $0.2 \mathrm{ppm}$ in the whole temperature range (Fig. 2-33). The presence of remaining LiTMP can be excluded since it resonances at $0.7 \mathrm{ppm}$ (monomer) or at $1.3 \mathrm{ppm}$ (dimer), respectively. ${ }^{[35 \mathrm{~b}]}$ Additionally, the lithium

\footnotetext{
${ }^{27} \mathrm{~A}$ small amount of protonated amine TMP(H) is also present in solution. The ECC-MW-determination predicts at temperatures between $0^{\circ} \mathrm{C}$ and $-75^{\circ} \mathrm{C}$ an accurate $\mathrm{MW}$ (in av.: $\mathrm{MW}_{\text {calc }}=141 \mathrm{~g} / \mathrm{mol}, \mathrm{MW}_{\text {det }}=$ $\left.140 \mathrm{~g} / \mathrm{mol}, \mathrm{MW}_{\mathrm{err}}=1 \%\right)$. Interestingly, at RT the $\mathrm{MW}$ is slightly overestimated $\left(\mathrm{MW}_{\mathrm{det}}=167 \mathrm{~g} / \mathrm{mol}, \mathrm{MW}_{\mathrm{err}}=\right.$ $-18 \%$ ), indicating an small exchange between the amide and the amine. Anyway, this interaction is neglectible at temperatures below $0^{\circ} \mathrm{C}$, see $\mathrm{A}$-Table 32 in the appendix.
} 
cation $\left[\mathrm{Li}(\mathrm{THF})_{4}\right]^{+} \mathbf{L i 2}$ in a SSIP can also be excluded to be present at significant concentrations since it is known to resonate at negative field $(-1.1 \mathrm{ppm}),{ }^{[128]}$ curtailing the plausible present species to the lithium containing dimer $\mathbf{1 A}$ and the monomers $\mathbf{1 C} / \mathbf{1} \mathbf{C}^{\star}$. Both aggregates should clearly be distinguishable via DOSY NMR spectroscopy

The ${ }^{1} \mathrm{H}$ and ${ }^{7} \mathrm{Li}$-DOSY-ECC-MW-determination of 10 gives a $\mathrm{MW}$ of $\mathrm{MW}_{\text {det }}=403 \mathrm{~g} / \mathrm{mol}$ for the ${ }^{1} \mathrm{H}$ - und $387 \mathrm{~g} / \mathrm{mol}$ for the ${ }^{7} \mathrm{Li}$-nucleus (Fig. 2-34 vide infra). ${ }^{28}$ This low $\mathrm{MW}$ discriminates both the lithium free aggregates 1B $\left(\mathrm{MW}_{\mathrm{err}}=26 \%\right), 1 \mathrm{D}\left(\mathrm{MW}_{\mathrm{err}}=-17 \%\right), \mathbf{E}$ $\left(\mathrm{MW}_{\mathrm{err}}=-31 \%\right)$ and also dimeric 1A $\left(\mathrm{MW}_{\mathrm{err}}=48 \%\right)$. Most interestingly the crystal structure 1C does not keep its full integrity in THF- $d_{8}$ solution reflected by an unacceptable high error of the $\mathrm{MW}\left(\mathrm{MW}_{\text {calc }}=459 \mathrm{~g} / \mathrm{mol}, \mathrm{MW}_{\text {det }}=403 \mathrm{~g} / \mathrm{mol}, \mathrm{MW}_{\mathrm{err}}=\right.$ $12 \%)$. The smaller contact ion pair $1 \mathrm{C}^{\star}$ derived from $\mathbf{1 C}$ by the loss of a single THF molecule matches best the determined $\mathrm{MW}\left(\mathrm{MW}_{\text {calc }}=387 \mathrm{~g} / \mathrm{mol}, \mathrm{MW}_{\text {det }}=403 \mathrm{~g} / \mathrm{mol}\right.$, $\left.\mathrm{MW}_{\mathrm{err}}=-4 \%\right)$. Mulvey et al. already mentioned the labile THF coordination at $\mathbf{1 C}$ as the expected integrated ${ }^{1} \mathrm{H}$ NMR intensity THF:amide decreases from 3:1 to 2:1 when the crystals are dried in vacuo. ${ }^{[72]}$ From that they concluded that the powerful regioselective magnesiating ability of $\mathbf{1 0}$ might be a consequence of a coordinately unsaturated $\mathrm{Mg}$ that "could facilitate the pre-coordination of the functionalized aromatic substrate prior to magnesiation". ${ }^{772]}$

Our DOSY-ECC-MW-determination fully supports this hypothesis. The labile THF ligand at the magnesium atom could be a result of steric overload from the rigid TMP ligand. Searching the Cambridge Crystallographic Database ${ }^{29}$ emphasizes Mg typically to be coordinated by four to six ligands. Enlarging the bulk of the various substituents however results also in 3-coordinated magnesium amides. This is often the case when bulky $\mathrm{SiMe}_{3}$ groups $^{[135]}$ and especially when TMP ligands are involved. ${ }^{[105 a, 136]}$ These investigations confirm that lithium chloride indeed co-coordinates to the magnesium amides in solution as observed in the ${ }^{i} \mathrm{Pr}$-Turbo-Hauser base 9. ${ }^{[137]}$ A detactable population of the solvent separated lithium cation Li2 can also be excluded for Turbo-Hauser base $\mathbf{1 0}\left(M W_{\text {calc }}=\right.$ $\left.295 \mathrm{~g} / \mathrm{mol}, M W_{\text {det }}=387 \mathrm{~g} / \mathrm{mol}, M W_{\text {err }}=-31 \%\right)$.

\footnotetext{
${ }^{28}$ The $\mathrm{MW}_{\text {det }}$ values are dispayed as an average value, derived from DOSY-ECC-MW measurements at different temperatures, see A-Table 30 and A-Table 31 in the appendix.

${ }^{29}$ CSD version 5.36 (Updated Nov 2014)
} 


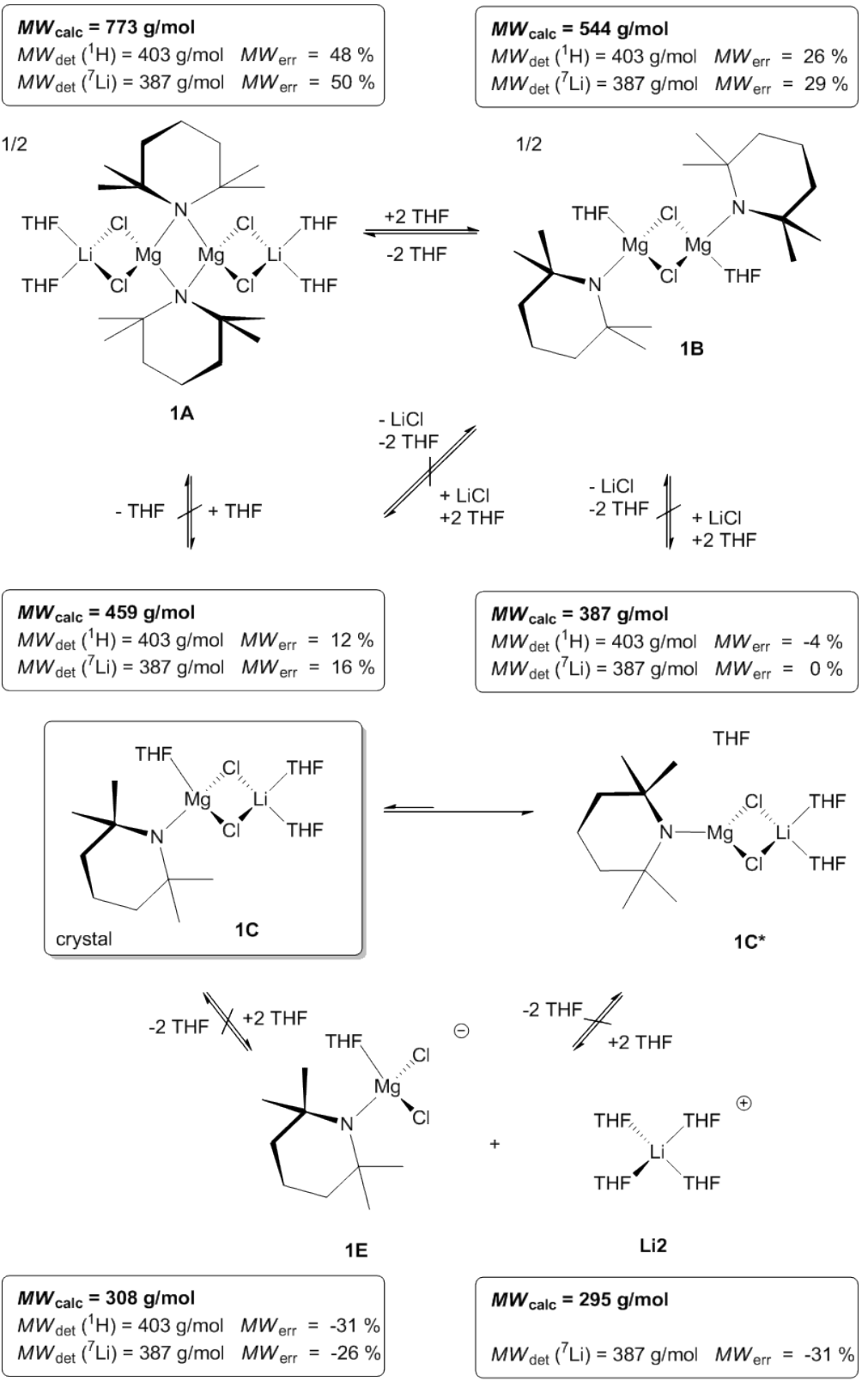

Fig. 2-34. ${ }^{1} \mathrm{H}$ - and ${ }^{7} \mathrm{Li}$-DOSY-ECC-MW-determination of crystalloid 10 re-dissolved in THF- $d_{8}(20 \mathrm{~mm})$. 


\section{SUMMARY AND OUTLOOK}

In the first part of this $\mathrm{Ph}$. D. thesis the new DOSY-ECC-MW-determination methodology was introduced. This method determines molecular weights of unknown solutes in THF- $d_{8}$ and TOL- $d_{8}$ solution with exceptional accurately und reliability with a maximum deviation of $\pm 9 \%$ and an average error of only $4 \%$ (Table $3-1$ ). Furthermore, the influence of the internal reference, shape, concentration, temperature, heavy atoms and deuterated compounds on the MW-determination was validated in detail. ${ }^{[6]}$ Due to the normalized diffusion coefficients one internal reference is sufficient. Compared to the ICC method, this interrelation has the huge advantage that it is unnecessary to introduce multiple references into the same NMR sample. Signal overlapping, analyte-reference interaction problems, wasting chemicals and deuterated solvents can be avoided. Due to the normalized diffusion coefficients everyone is able to use the ECCs, independent of the NMR device, without the necessity of recording new calibration curves. This work facilitated consecutive research in which new ECCs for a range of further commonly used NMR solvents like DMSO- $d_{6}, \mathrm{C}_{6} \mathrm{D}_{12}, \mathrm{C}_{6} \mathrm{D}_{6}, \mathrm{CDCl}_{3}$ and $\mathrm{CD}_{2} \mathrm{Cl}_{2}$ were introduced. ${ }^{[4]}$ Additionally, future investigations will include the preparation of ECCs derived from molecules with high densities which contain e.g. heavy halides or transition metals. This extension could promote the ECC-method into a widely applicable standard technique to elucidate solution state structures. ${ }^{30}$

Table 3-1. RMS errors for DOSY-MW-determination of small molecules by different methods.

\begin{tabular}{cc}
\hline Method & RMS error / \% \\
\hline Stokes-Einstein $^{[101]}$ & 45 \\
Gierer-Wirtz $^{[101]}$ & 28 \\
Chen $^{[101]}$ & 18 \\
Evans $^{[101]}$ & 15 \\
Crutchfield $^{[103]}$ & 11 \\
ECC $^{[6]}$ & 4
\end{tabular}

\footnotetext{
${ }^{30} \mathrm{~A}$ simple Excel spreadsheet that implements the calculation of $\log D_{\mathrm{x}, \mathrm{norm}}$, allowing to estimate MWs of analytes from their diffusion coefficients is available at http://www.stalke.chemie.uni-goettingen.de/ mw_det_calc/mw_det_calc.xlsx
} 
In the second part of this Ph. D. thesis DOSY-ECC-MW-determinations were performed on several organometallic and metal amide compounds. For the first time it was possible to determine the donor-base-free solution structure of LDA in toluene (Fig. 3-1). ${ }^{[5]}$ It was shown that at room temperature LDA adopts a trimeric and tetrameric aggregation in a 2:1 ratio. This equilibrium ranges from trimers and tetramers through pentamers to higher oligomers as the temperature decreases. ${ }^{[5]}$ Additionally, it was shown that dimeric LDA as proposed by Kim and Collum et al. ${ }^{[12 a]}$ is not present in the mixture at any temperature $\left(M W_{\text {err }}=-48 \%\right.$ and $\left.-143 \%\right)$.
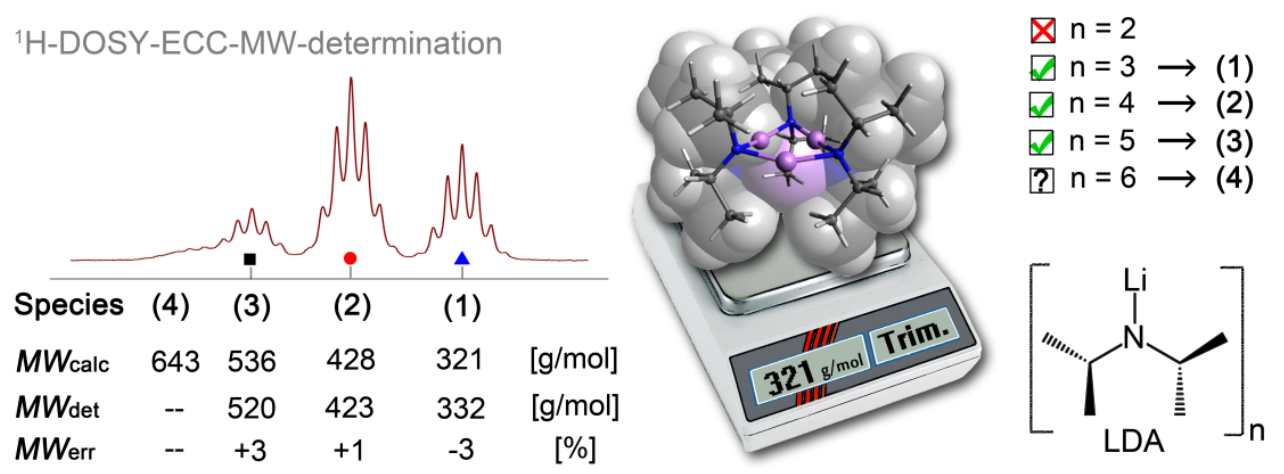

Fig. 3-1. Illustration of the DOSY-ECC-MW-determination of LDA in TOL- $d_{8}$ solution.

The ECC-approach was further used to determine the aggregation of Na-indenide. It was shown that Na-indenide is predominately a trisolvated monomer $(\mathrm{THF})_{3} \cdot \mathrm{Na}\left(\mathrm{C}_{9} \mathrm{H}_{7}\right)$ in THF- $d_{8}$ solution (Fig. 3-2). ${ }^{[6]}$ With decreasing temperature the MW increases. This behavior was attributed to a fourth THF coordination which seems to be attractive at low temperatures.
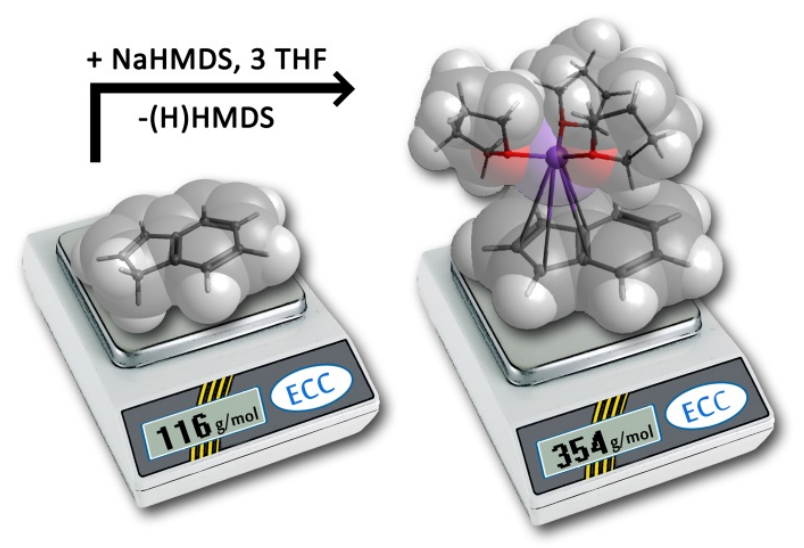

Fig. 3-2. Illustration of the DOSY-ECC-MW-determination of Na-indenide in THF- $d_{8}$ solution. 
By taking the residual proton signal of the solvent (TOL- $d_{7}$ ) as internal reference it was possible to characterize a posteriori the complex solution structure of $\left[{ }^{t} \mathrm{BuLi}\right]_{4} \cdot 4\left[\mathrm{Me}_{2} \mathrm{NC}_{6} \mathrm{H}_{4} \mathrm{Li}\right]_{4}$ in TOL- $d_{8}{ }^{[6]}$ The results from the DOSY-ECC-MWdetermination correlate very well (error smaller than $\pm 5 \%$ ) with the solution structures that were determined in a ${ }^{7} \mathrm{Li}$-EXSY NMR experiment by A.-C. Pöppler et al. in 2012. ${ }^{[19]}$

Dimeric solid state structures of MHMDS (65-68 and 71 in Fig. 3-3) with ammonia as donor base were presented and an aggregation-deaggregation mechanism was proposed. Additionally, MW-determinations on Li-, Na- and KHMDS were performed in TOL- $d_{8}$ solution at room temperature. The measurements indicate that the latter form predominately mono- to penta- solvated dimers.

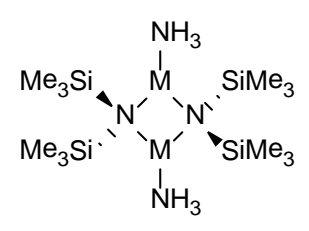

$65 \mathrm{M}=\mathrm{Li}$

$66 \mathrm{M}=\mathrm{Na}$

$67 \mathrm{M}=\mathrm{K}$

$68 M=R b$

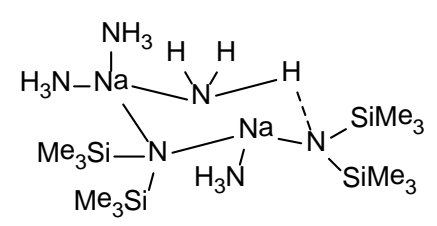

71

Fig. 3-3. Dimeric structure of MHMDS ( $\mathrm{M}=\mathrm{Li}, \mathrm{Na}, \mathrm{K}$ and $\mathrm{Rb})$ with $\mathrm{NH}_{3}$ in the solid state.

However, several aspects remain to be investigated in more detail. It should be proved how the concentration of $\mathrm{NH}_{3}$ and MHMDS and the temperature influence the solution structures. The concentration of ammonia could be easily increased e.g. by introducing gaseous ammonia at low temperature to the NMR-sample. Finally, it would be also interesting to investigate the solution structures of the heavier analogues $\mathrm{Rb}$ - and CsHMDS.

Furthermore, single crystals of Hauser base ${ }^{i} \operatorname{Pr}_{2} \mathrm{NMgCl} 7$ and Turbo Hauser base ${ }^{i} \operatorname{Pr}_{2} \mathrm{NMgCl} \cdot \mathrm{LiCl} 9$ were grown and analyzed in THF solution by DOSY-ECC-MWdeterminations. The results show that their aggregation in THF- $d_{8}$ differs significantly and that the solution composition of the existing species is highly temperature dependent. ${ }^{[137]}$ Knowing the position of the equilibrium is of essential importance for every synthetic chemist, since Hauser bases as well as their Turbo analogues are used in synthesic protocols at various temperatures $\left(-75^{\circ} \mathrm{C}\right.$ to $\left.25^{\circ} \mathrm{C}\right) .{ }^{[16,23]}$ The solution structure of 7 is best represented by the common Schlenk-equilibrium with heteroleptic M1 as the main species 
at high temperatures and homoleptic M2 at low temperatures (Fig. 3-4a). However, it was also shown that in Hauser base 7 dimeric species are also present in the THF solution although alkyl magnesium chlorides do not dimerize in that solvent. Therefore, the Hauser base 7 Schlenk-equilibrium has to be extended to dimeric amido bridged species and additionally to $\mathrm{MgCl}_{2}$ co-coordinated species which exist only at low temperatures, where an excess of $\mathrm{MgCl}_{2}$ is present.

a)

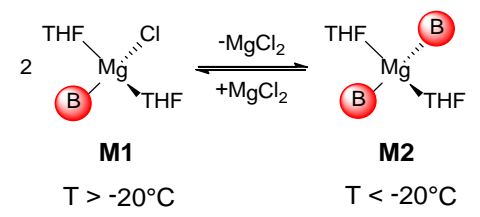

b)
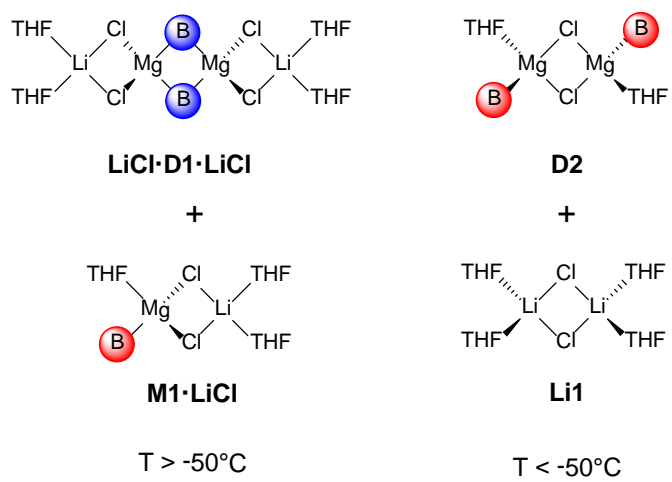

Fig. 3-4. Main species of a) Hauser base ${ }^{i} \mathrm{Pr}_{2} \mathrm{NMgCl} 7$ and b) Turbo-Hauser base ${ }^{i} \mathrm{Pr}_{2} \mathrm{NMgCl} \cdot \mathrm{LiCl} 9$ in THF$d_{8}$ solution with $\mathrm{B}={ }^{i} \operatorname{Pr}_{2} \mathrm{~N}^{-} \cdot{ }^{[137]}$

It was then demonstrated that the addition of $\mathrm{LiCl}$ to 7 has an enormous impact on the Schlenk-equilibrium. The hypothesis that the impact of $\mathrm{LiCl}$ on the higher reactivity of Turbo bases rests on the deaggregation of $\mathrm{RMgX}$ oligomers to monomers ${ }^{[23,28]}$ has to be revised. Moreover, the main advantage of $\mathrm{LiCl}$ is the ability to shift the Schlenkequilibrium from the homoleptic to the heteroleptic side. At RT monomeric $\mathbf{M l} \cdot \mathbf{L i C l}$ and dimeric $\mathbf{~ L i C l \cdot D 1 \cdot L i C l}$ are the main species in solution of $\mathbf{9}$ (Fig. 3-4b), while the letter is the most populated species at high concentration $(0.5$ to $0.6 \mathrm{M})$. Lowering the temperature below $-50^{\circ} \mathrm{C}$ results in the formation of $\mathbf{D} 2$ and the LiCl-dimer Li1. The latter stabilizes the heteroleptic dimer D2 and inhibits the formation of homoleptic $\left({ }^{i} \operatorname{Pr}_{2} \mathrm{~N}\right){ }_{2} \mathrm{Mg}$ (M2) and $\mathrm{MgCl}_{2}$. It is possible that this concept could also be applied to Turbo-Grignard reagents. 
Finally, the structure of Turbo-Hauser base TMPMgCl$\cdot \mathrm{LiCl} 10$ was investigated in THF- $d_{8}$ solution. DOSY-ECC-MW-determinations and ${ }^{1} \mathrm{H}-{ }^{7} \mathrm{Li}-\mathrm{HOESY}$ experiments confirm that lithium chloride indeed co-coordinates to the magnesium amides in solution as observed in the Turbo-Hauser base 9. ${ }^{[137]}$ A detactable population of the solvent separated lithium cation Li2 can also be excluded for Turbo-Hauser base $10\left(M W_{\text {calc }}=295 \mathrm{~g} / \mathrm{mol}, M W_{\text {det }}=\right.$ $\left.387 \mathrm{~g} / \mathrm{mol}, M W_{\text {err }}=-31 \%\right)$.

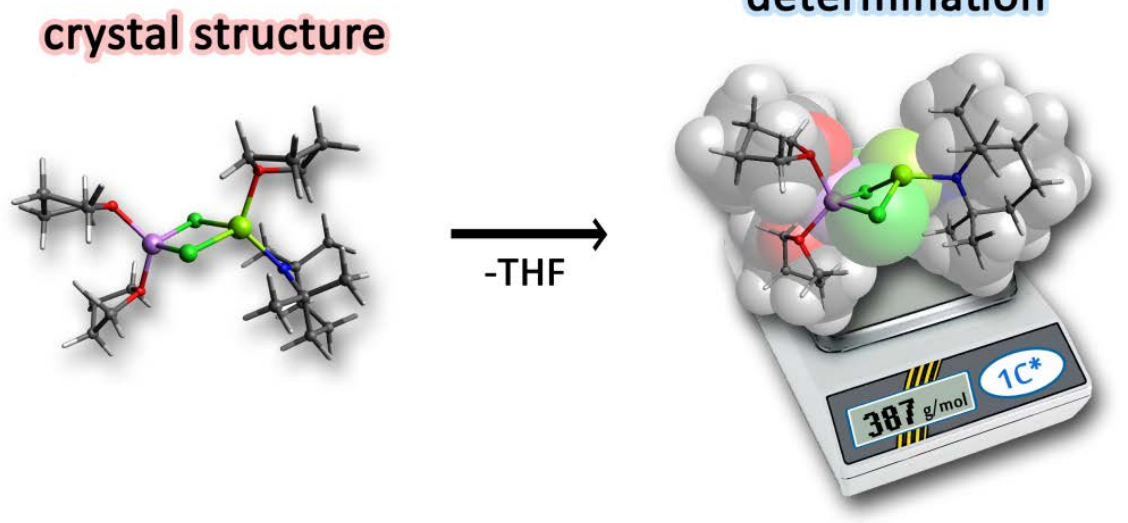

DOSY-ECC-MWdetermination

Fig. 3-5. Illustration of the DOSY-ECC-MW-determination of Turbo-Hauser base TMPMgCl.LiCl 10 in $\mathrm{THF}-d_{8}$ solution.

The results show that changing the steric demand and the electronic properties of the amide strongly controls the structural features, both in the solid state as well as in solution. While $\mathbf{1 0}$ is a monomer in the solid state and in solution, $\mathbf{9}$ is a dimer in the crystal structure and forms predominately dimeric aggregates in THF-solution. ${ }^{[137]}$ From our measurements the different reactivities can be rationalized as followed: The TMP ligand in TMPMgCl. LiCl 10 is bulky enough to prevent dimerization and to promote a $\mathrm{LiCl}$ solubilized monomer $\mathbf{1 C}^{\star}$ (Fig. 3-5). ${ }^{[137]}$ Similarly the considerable steric demand provided by the fixed methyl groups facilitates the cleavage of a labile THF ligand and induces an unsaturated magnesium site. $\operatorname{In}{ }^{i} \mathrm{Pr}_{2} \mathrm{NMgCl} \cdot \mathrm{LiCl} 9$ the rigid TMP ligand is replaced by two floppy diisopropyl groups and the methyl groups are not fixed in a static position relative to the metal. This flexibility permits both, a direct THF coordination at the Mg cation and a dimerization of the lithium stabilized monomer $\mathbf{M 1} \cdot \mathbf{L i C l}$ to form the tetranuclear dimer LiCl.D1·LiCl. The latter is at high concentrations the main aggregate in solution. ${ }^{[28,137]}$ That would also explain the limited solubility of dimeric 9 in THF $(0.6 \mathrm{M})$ compared to the TMP-Turbo-Hauser base $10(1.2 \mathrm{M})$ that is monomeric at all concentrations. Therefore, reactions of $\mathbf{1 0}$ should predominately involve a monomeric and those of $\mathbf{9}$ mainly a dimeric attack at the reactant. ${ }^{[137]}$ Beside the unsaturated magnesium atom in $\mathbf{1 0}$ this explains the 
extreme high reactivity of $\mathbf{1 0}$, reminiscent of the general higher reactivity of monomeric organometallics in comparison to their oligomeric analogues. ${ }^{[138]}$

In summary, this work illustrates that the DOSY-ECC-method adds profound conceptional value to study aggregation in solution. Additionally, the presented organometallic solution structures provide details on reaction conditions which should have a tremendous impact on modified reaction protocols. 


\section{EXPERIMENTAL PART}

\subsection{Techniques and Experiments}

\subsubsection{Handling of Air- and Moisture Sensitive Compounds}

All air- and moisture sensitive compounds were manipulated with standard Schlenk techniques ${ }^{[139]}$ either in an inert atmosphere of purified and dry argon or in an argon glove box. The glassware was dried at $140^{\circ} \mathrm{C}$, assembled hot and cooled down under high vacuum. All solvents were dried with standard drying techniques over sodium, potassium or sodium-potassium alloy and were distilled prior to use.

\subsubsection{NMR Experiments}

NMR experiments were recorded either on Bruker Avance 400 spectrometer equipped with an observe broadband probe with $\mathrm{z}$-axis gradient coil with maximum gradient strength of $57 \mathrm{G} / \mathrm{cm}$ or on Bruker Ascend 400 spectrometer equipped with an inverse broadband probe with $\mathrm{z}$-axis gradient coil with maximum gradient strength of $51 \mathrm{G} / \mathrm{cm}$. All spectra were acquired using $5 \mathrm{~mm}$ NMR tubes, which were not spun during the measurements. Chemical shifts $(\delta)$ are given in ppm relative to TMS using the residual solvent signals as internal standards. Coupling constants $(\mathrm{J})$ are reported in $\mathrm{Hz}$ and standard abbreviations indicating multiplicity are used as follows:

$\mathrm{s}=$ singlet, $\mathrm{d}=$ doublet $\mathrm{t}=$ triplet, sept $=$ septet, $\mathrm{m}=$ multiplet and $\mathrm{br}=$ broad .

\subsubsection{DOSY-NMR-Experiments}

Dry TOL- $d_{8}$ or THF- $d_{8}$ kept with $4 \AA$ molecular sieves under argon was used. The NMR samples were prepared by solving either purified or crystalline compounds $(0.015-0.120 \mathrm{M})$ and a DOSY reference under argon atmosphere in the deuterated NMR solvent. In the case of Li-, $\mathrm{Na}-$, and KHMDS gaseous $\mathrm{ND}_{3}$ was introduced to the solution (approximately one minute at $+25^{\circ} \mathrm{C}$ ). Finally, the NMR-tubes were sealed and DOSY measurements were performed at RT. For all DOSY-NMR measurements the diffusion coefficient of the analyte was normalized to the fixed diffusion value of the internal reference. All DOSY experi- 
ments were performed using a double stimulated echo sequence with bipolar gradient pulses and three spoil gradients with convection compensation (dstebpgp3s). ${ }^{[91,96]}$ The duration of the magnetic field pulse gradients was adjusted for every temperature in a range of $\delta / 2=400-3500 \mu \mathrm{s}$. The diffusion time was $\Delta=0.1 \mathrm{~s}$. The delay for gradient recovery was $0.2 \mathrm{~ms}$ and the eddy current delay $5 \mathrm{~ms}$. In each PFG NMR experiment, a series of 16 spectra on $32 \mathrm{~K}$ data points were collected. The pulse gradients were incremented from 2 to $98 \%$ of the maximum gradient strength in a linear ramp. After Fourier transformation and baseline correction, the diffusion dimension was processed with the Topspin 3.1 software. Diffusion coefficients, processed with a line broadening of $2 \mathrm{~Hz}$, were calculated by Gaussian fits with the T1/T2 software of Topspin.

\subsubsection{Computational Details}

All structures included in this $\mathrm{Ph}$. D. thesis were optimized at the B3LYP-D3/def2-SVP level of theory ${ }^{[122]}$ (the dispersion corrections were computed with Becke-Johnson type damping). ${ }^{[140]}$ The electronic energies were recomputed with the def2-TZVP basis set. ${ }^{[122 b]}$ Solvation effects were included through the use of the COSMO continuum model ${ }^{[123]}$ both in the energy and optimization runs. All stationary points were confirmed to be true minima on the potential energy surface through harmonic vibrational calculations. All thermodynamic correction terms were derived from the latter. In order to deal with the large errors associated with low-energy vibrational modes (particularly in large complexes) we applied the quasi-rigid rotor harmonic oscillator formula proposed by Grimme, ${ }^{[141]}$ with a cutoff parameter of $100 \mathrm{~cm}^{-1}$. Furthermore, corrections were included to the entropy to account for the overestimation of translational freedom in solution. The latter was included taking a cell model for the change in translational degrees of freedom. ${ }^{[134]}$ All reported energy values, unless otherwise noted, correspond to free energies. All calculations were carried out with the Orca 3.0.3 program package. ${ }^{[142]}$ 


\subsection{Synthesis and Crystallization}

\subsubsection{Donor-Base-Free LDA}

Diisopropyl amine (15.58 g, $0.15 \mathrm{~mol}, 1.07$ equiv) was dissolved in dry pentane $(150 \mathrm{~mL})$. At $0{ }^{\circ} \mathrm{C}{ }^{n} \mathrm{BuLi}(5.64 \mathrm{~mol} / \mathrm{L}, 25 \mathrm{~mL}, 0.14 \mathrm{~mol}, 1.00$ equiv) was added dropwise to the solution. After 20 min the reaction mixture was warmed up to RT and then stirred for $1 \mathrm{~h}$. The reaction mixture was then slowly cooled down to $-78^{\circ} \mathrm{C}$. After $3 \mathrm{~h}$ the mother liquor was removed via a syringe. Finally the solvent was evaporated at RT under vacuum (approx. $6 \mathrm{~h})$ to afford 4 as a white solid (10.41 g, $0.10 \mathrm{~mol}, 71 \%)$.

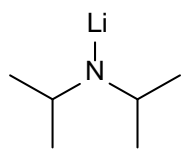

4

${ }^{1} \mathrm{H}-\mathrm{NMR}$

(400.13 MHz, THF- $\left.d_{8}, 25^{\circ} \mathrm{C}\right)$ :

${ }^{7} \mathrm{Li}-\mathrm{NMR}$

$\left(155.51 \mathrm{MHz}, \mathrm{THF}-d_{8}, 25^{\circ} \mathrm{C}\right)$ :

${ }^{13} \mathrm{C}\left\{{ }^{1} \mathrm{H}\right\}-\mathrm{NMR}$

$\left(100.62 \mathrm{MHz}, \mathrm{THF}-d_{8}, 25^{\circ} \mathrm{C}\right)$ : $\delta(\mathrm{ppm})=2.03(\mathrm{~s})$

$$
\delta(\mathrm{ppm})=26.7\left(\mathrm{~s},-\mathrm{CH}_{3}\right), 51.1(\mathrm{~s},-\mathrm{CH}) .
$$




\subsubsection{Na-Indenide}

NaHMDS (0.73 g, $4.00 \mathrm{mmol}, 1.00$ equiv) was dissolved in dry pentane (22 mL). At RT indene $(0.56 \mathrm{~g}, 4.80 \mathrm{mmol}, 1.20$ equiv) was added dropwise and the reaction mixture was warmed up to $+70^{\circ} \mathrm{C}$ and stirred for $30 \mathrm{~min}$ at this temperature. Finally, the product was washed at RT with pentane (2x $15 \mathrm{~mL}$ ) and dried under vacuum (approx. $6 \mathrm{~h}$ ) to afford 73 as reddish solid $(0.45 \mathrm{~g}, 3.24 \mathrm{mmol}, 81 \%)$.

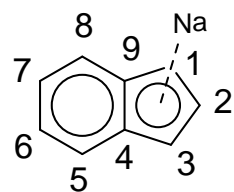

${ }^{1} \mathrm{H}-\mathrm{NMR}$

$\left(400.13 \mathrm{MHz}\right.$, THF- $\left.d_{8}, 25^{\circ} \mathrm{C}\right): \quad \delta(\mathrm{ppm})=5.90\left(\mathrm{~d},{ }^{3} \mathrm{~J}_{\mathrm{HH}}=3.1 \mathrm{~Hz}, 2 \mathrm{H}, \mathrm{H} 1 / \mathrm{H} 3\right), 6.36$

$\left(\mathrm{m}, 2 \mathrm{H}, \mathrm{H} 6 / \mathrm{H} 7,6.56\left(\mathrm{t},{ }^{3} \mathrm{~J}_{\mathrm{HH}}=3.0 \mathrm{~Hz}, 1 \mathrm{H}, \mathrm{H} 2\right), 7.27\right.$

(m, 2H, H5/H8).

${ }^{13} \mathrm{C}\left\{{ }^{1} \mathrm{H}\right\}-\mathrm{NMR}$

$\left(100.62 \mathrm{MHz}, \mathrm{THF}-d_{8}, 25^{\circ} \mathrm{C}\right): \quad \delta(\mathrm{ppm})=91.2(\mathrm{~s}, \mathrm{C} 1, \mathrm{C} 3), 112.4(\mathrm{~s}, \mathrm{C} 5, \mathrm{C} 8), 115.5(\mathrm{~s}$, C2), 118.3 (s, C6, C7), 128.4 (s, C4, C9).

\subsubsection{MHMDS-Ammoniacates ( $\mathrm{M}=\mathrm{Li}, \mathrm{Na}, \mathrm{K}, \mathrm{Rb}$ and $\mathrm{Cs}$ )}

Each of the described complexes was prepared in a similar manner. MHMDS ( $\mathrm{m}=0.5 \mathrm{~g})$ was kept under argon atmosphere due to its hygroscopic character. After cooling the flask to $-78^{\circ} \mathrm{C}$ gaseous ammonia (approx. $15 \mathrm{~mL}$ ) was condensed in the crystallization Schlenk flask till all MHMDS was dissolved. After dissolving, the ammonia solution was overfilled with dry toluene $(15 \mathrm{~mL})$. Finally the colorless solution was warmed to $+25^{\circ} \mathrm{C}$ so excess ammonia could evaporate. Afterwards storage at $-45^{\circ} \mathrm{C}$ afforded colorless crystals of $65-68$ within some days which finally were suitable for X-ray structure analysis (see chapter 4.4.1 to 4.4.5). Intermediate 71 was crystallized from a solution which was warmed up to $0^{\circ} \mathrm{C}$ instead of $+25^{\circ} \mathrm{C}$. Unfortunately, the crystals were not stable at temperatures higher than $-33^{\circ} \mathrm{C}$. This is why it was not possible to investigate in NMR measurements of re-dissolved ammoniacate crystals. 


\subsection{4 ${ }^{i} \mathrm{Pr}_{2} \mathrm{NMgCl}$}

${ }^{i} \mathrm{PrMgCl}(62.10 \mathrm{mmol}, 31.05 \mathrm{~mL}, 2 \mathrm{M}$ in THF, 1.0 equiv) was dropped to bulk diisopropylamine ${ }^{i} \operatorname{Pr}_{2} \mathrm{NH}$ (68.31 mmol, $9.60 \mathrm{~mL}, 1.1$ equiv) at RT. Stirring overnight and removal of the solvent in vacuo yielded an offwhite powder $(11.68 \mathrm{~g}, 96 \%$, considering the loss of one molecule of THF). To receive crystalline product a saturated THF solution $(24 \mathrm{~mL})$ of $[7 \cdot \mathrm{THF}]_{2}$ was prepared at RT. After reducing the solvent, addition of $5 \mathrm{~mL}$ toluene and storage at $-6^{\circ} \mathrm{C}[7 \cdot \mathrm{THF}]_{2}$ was isolated as colourless crystals which were suitable for single crystal X-ray analysis (see chapter 4.4.6).

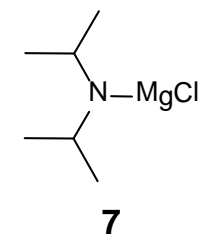

${ }^{1} \mathrm{H}-\mathrm{NMR}$

(400.13 MHz, THF- $\left.d_{8}, 25^{\circ} \mathrm{C}\right)$ :

${ }^{13} \mathrm{C}\left\{{ }^{1} \mathrm{H}\right\}-\mathrm{NMR}$

$\left(100.62 \mathrm{MHz}, \mathrm{THF}-d_{8}, 25^{\circ} \mathrm{C}\right)$ : $\delta(\mathrm{ppm})=1.01\left(\mathrm{~d},{ }^{3} \mathrm{~J}_{\mathrm{HH}}=6.1 \mathrm{~Hz}, 12 \mathrm{H},-\mathrm{CH}_{3}\right), 2.94$ (sept, ${ }^{3} \mathrm{~J}_{\mathrm{HH}}=6.4 \mathrm{~Hz}, 2 \mathrm{H},-\mathrm{CH}$ ).

$\delta(\mathrm{ppm})=26.9\left(\mathrm{~s},-\mathrm{CH}_{3}\right), 50.8(\mathrm{~s},-\mathrm{CH})$.

\subsection{5 ${ }^{i} \mathrm{Pr}_{2} \mathrm{NMgCl} \cdot \mathrm{LiCl}$}

In a modified preparation route of Armstrong, ${ }^{[28]}$ the Turbo-Hauser base $[\mathbf{9} \cdot \mathrm{THF}]_{2}$ was synthesized by reaction of LDA ( $0.43 \mathrm{~g}, 4.00 \mathrm{mmol}, 1.0$ equiv) and a suspension of $\mathrm{MgCl}_{2}$ $(0.38 \mathrm{~g}, 4.00 \mathrm{mmol}, 1.0$ equiv) in dry THF $(10 \mathrm{~mL})$ and stirring the mixture at RT overnight. Removing the solvent in vacuo and recrystallization (2x) in a 1:1 mixture of $\mathrm{THF} /$ hexane at $-45^{\circ} \mathrm{C}$ afforded $[\mathbf{9} \cdot \mathrm{THF}]_{2}$ as colourless crystals. The mother liquor was removed via a syringe at $-45^{\circ} \mathrm{C}$ and the crystals were washed with cold hexane $(2 \times 10 \mathrm{~mL})$. Finally, the structure was proven via X-Ray diffraction: Space group $P 2_{1} / n ; a=10.317 \AA$, $b=16.658 \AA, c=11.859 \AA ; \beta=109.14^{\circ}{ }^{[28]}$

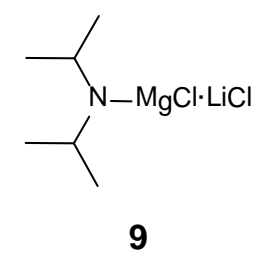

${ }^{1} \mathrm{H}-\mathrm{NMR}$

$\left(400.13 \mathrm{MHz}, \mathrm{THF}-d_{8}, 25^{\circ} \mathrm{C}\right)$ :
Monomer:

$\delta(\mathrm{ppm})=1.03\left(\mathrm{~d},{ }^{3} \mathrm{~J}_{\mathrm{HH}}=6.2 \mathrm{~Hz}, 12 \mathrm{H},-\mathrm{CH}_{3}\right), 2.93$ 
(sept, ${ }^{3} \mathrm{~J}_{\mathrm{HH}}=6.1 \mathrm{~Hz}, 2 \mathrm{H},-\mathrm{CH}$ ).

Dimer:

$\delta(\mathrm{ppm})=1.33\left(\mathrm{~d},{ }^{3} \mathrm{~J}_{\mathrm{HH}}=5.5 \mathrm{~Hz}, 12 \mathrm{H},-\mathrm{CH}_{3}\right), 3.42$ (br, $2 \mathrm{H},-\mathrm{CH})$.

${ }^{7} \mathrm{Li}-\mathrm{NMR}$

$\left(155.51 \mathrm{MHz}, \mathrm{THF}-d_{8}, 25^{\circ} \mathrm{C}\right): \quad \delta(\mathrm{ppm})=0.18(\mathrm{~s})$

${ }^{13} \mathrm{C}\left\{{ }^{1} \mathrm{H}\right\}-\mathrm{NMR}$

$\left(100.62 \mathrm{MHz}, \mathrm{THF}-d_{8}, 25^{\circ} \mathrm{C}\right): \quad \delta(\mathrm{ppm})=27.4\left(-\mathrm{CH}_{3}\right), 48.1(-\mathrm{CH}), 53.2(-\mathrm{CH})$.

\subsubsection{TMPMgCl·LiCl}

In a modified preparation route of Alvarez, ${ }^{[72]}$ the Turbo-Hauser base 10.(THF) ${ }_{3}$ was synthesized by reaction of LiTMP ( $0.59 \mathrm{~g}, 4.00 \mathrm{mmol}, 1.0$ equiv) and a suspension of $\mathrm{MgCl}_{2}$ ( $0.38 \mathrm{~g}, 4 \mathrm{mmol}, 1.0$ equiv) in dry THF $(10 \mathrm{~mL})$ and stirring the mixture at RT overnight. Removing the solvent in vacuo and recrystallization $(2 \mathrm{x})$ in a 1:1 mixture of THF/hexane at $-45^{\circ} \mathrm{C}$ afforded 10.(THF) $)_{3}$ as colourless crystals. The mother liquor was removed via a syringe at $-45^{\circ} \mathrm{C}$ and the crystals were washed with cold hexane $(2 \times 10 \mathrm{~mL})$. Finally, the structure was proven via X-Ray diffraction: Space group $P 2_{1} / c ; a=8.313 \AA, b=26.214 \AA$, $c=14.116 \AA ; \beta=120.53^{\circ} .^{[72]}$

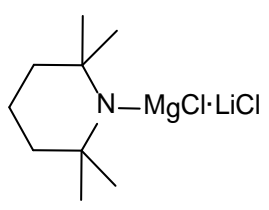

10

${ }^{1} \mathrm{H}-\mathrm{NMR}$

(400.13 MHz, THF- $\left.d_{8}, 25^{\circ} \mathrm{C}\right)$ :

$\delta(\mathrm{ppm})=1.18\left(\mathrm{~s}, 12 \mathrm{H},-\mathrm{CH}_{3}\right), 1.20\left(\mathrm{t},{ }^{3} \mathrm{~J}_{\mathrm{HH}}=6.1 \mathrm{~Hz}\right.$, $\left.4 \mathrm{H}, \beta-\mathrm{CH}_{2}\right), 1.60\left(\mathrm{~m}, 2 \mathrm{H}, \gamma-\mathrm{CH}_{2}\right)$.

${ }^{7} \mathrm{Li}-\mathrm{NMR}$

$\left(155.51 \mathrm{MHz}, \mathrm{THF}-d_{8}, 25^{\circ} \mathrm{C}\right): \quad \delta(\mathrm{ppm})=0.24(\mathrm{~s})$

${ }^{13} \mathrm{C}\left\{{ }^{1} \mathrm{H}\right\}-\mathrm{NMR}$

$\left(100.62 \mathrm{MHz}\right.$, THF- $\left.d_{8}, 25^{\circ} \mathrm{C}\right): \quad \delta(\mathrm{ppm})=21.0\left(\gamma-\mathrm{CH}_{2}\right), 36.2\left(-\mathrm{CH}_{3}\right), 43.0\left(\beta-\mathrm{CH}_{2}\right)$, $52.3\left(\alpha-\mathrm{CH}_{2}\right)$. 


\subsection{Preparation of DOSY-NMR-Samples}

\subsubsection{Internal References}

In THF- $d_{8}$ : Internal reference TMB and analyte (see A-Table 7 in the appendix) were dissolved in equimolar ratio (each $15 \mathrm{~mm}$ ) and DOSY measurements were performed immediately at $25^{\circ} \mathrm{C}$. Low temperature measurements were performed at -75 to $+60^{\circ} \mathrm{C}$ with TTS and ADAM as internal reference (each $15 \mathrm{~mm}$ ).

In TOL- $d_{8}$ : Internal reference ADAM and analyte (see A-Table 6 in the appendix) were dissolved in equimolar ratio (each $15 \mathrm{~mm}$ ) and DOSY measurements were performed immediately at $25^{\circ} \mathrm{C}$. For high concentration measurements $\mathrm{ADAM}$ and analytes were dissolved in equimolar ratio (each $120 \mathrm{~mm}$ ) to give a final concentration of $240 \mathrm{mM}$. Low temperature measurements were performed at -75 to $+100^{\circ} \mathrm{C}$ with TTS and ADAM as internal reference (each $15 \mathrm{~mm}$ ).

\subsubsection{LDA}

In THF- $d_{8}$ : Internal reference TMB and donor-base-free LDA (see chapter 4.2.1) were dissolved in equimolar ratio (each $15 \mathrm{~mm}$ ) and DOSY measurements were performed at $25^{\circ} \mathrm{C}$.

In TOL- $d_{8}$ : Internal reference ADAM and donor-base-free LDA (see chapter 4.2.1) were dissolved in equimolar ratio (each $15 \mathrm{~mm}$ ) and DOSY measurements were performed at $25^{\circ} \mathrm{C},-50^{\circ} \mathrm{C}$ and finally at $+100^{\circ} \mathrm{C}$.

\subsubsection{Na-Indenide}

Internal reference TMB and donor-base-free $\mathrm{Na}$-indenide (see chapter 0 ) were dissolved in THF- $d_{8}$ (each $15 \mathrm{~mm}$ ). DOSY spectra were collected at $25^{\circ} \mathrm{C}$ to $-50^{\circ} \mathrm{C}$ and finally at $+60^{\circ} \mathrm{C}$. 


\subsubsection{MHMDS-Ammoniacates $(\mathrm{M}=\mathrm{Li}, \mathrm{Na}$ and $\mathrm{K})$}

Internal reference ADAM and MHMDS were dissolved in TOL- $d_{8}$ (each $15 \mathrm{~mm}$ ). Under Schlenk conditions gaseous $\mathrm{ND}_{3}$ was introduced for approximately $1 \mathrm{~min}$ to the NMR sample at $25^{\circ} \mathrm{C}$. Finally, the NMR-tubes were sealed and DOSY measurements were performed at $25^{\circ} \mathrm{C}$.

\subsection{5 ${ }^{i} \mathrm{Pr}_{2} \mathrm{NMgCl}$ and ${ }^{\mathrm{i}} \mathrm{Pr}_{2} \mathrm{NMgCl} \cdot \mathrm{LiCl}$}

Internal reference $\mathrm{PhN}(20 \mathrm{~mm})$ and crystalline $[7 \cdot \mathrm{THF}]_{2}$ or $[\mathbf{9} \cdot \mathrm{THF}]_{2}(100 \mathrm{~mm})$ were dissolved in THF- $d_{8}$. DOSY spectra were collected at $25^{\circ} \mathrm{C}$ to $-100^{\circ} \mathrm{C}$.

\subsubsection{TMPMgCl·LiCl}

Internal reference $\mathrm{PhN}$ and crystalline 10.(THF) 3 (each $20 \mathrm{~mm}$ ) were dissolved in THF- $d_{8}$. DOSY spectra were collected at $25^{\circ} \mathrm{C}$ to $-75^{\circ} \mathrm{C}$.

\subsection{X-Ray Analysis}

Single crystals were selected due to their sensitivity and reactivity in inert perfluorinated oil. ${ }^{[143]}$ The X-ray data sets of $\mathbf{6 5}$ and $\mathbf{6 6}$ were collected at 100(2) K on a Bruker Smart Apex II Ultra diffractometer equipped with a Bruker Rotating Anode with Mo-K $\mathrm{K}_{\alpha}$ radiation $(\lambda=$ $0.71073 \AA$ ). The X-ray data sets of $[7 \cdot \mathrm{THF}]_{2}, \mathbf{6 7}, \mathbf{6 8}$ and 71 were collected at $100(2) \mathrm{K}$ on a Bruker Smart Apex II Quazar diffractometer with an Incoatec micro source ${ }^{[144]}$ equipped with mirror-monochromated Mo- $K_{\alpha}$ radiation $(\lambda=0.71073 \AA)$. The data were integrated with SAINT ${ }^{[145]}$ and a semi-empirical absorption correction with SADABS ${ }^{[146]}$ was applied. The structures were solved by direct methods with SHELXT ${ }^{[147]}$ and refined by full-matrix least-squares on $\mathrm{F}^{2}$ for all data with SHELXL. ${ }^{[148]}$ Non-hydrogen atoms were refined with anisotropic displacement parameters. Nitrogen-attached hydrogen atoms were located in the difference Fourier map and refined isotropically using distance similarity restraints for all $\mathrm{N}-\mathrm{H}$ and $\mathrm{H} \cdot \mathrm{H}-\mathrm{H}-1,2$-distances. All other hydrogen atoms were placed in calculated positions and refined using a riding model. ${ }^{[149]}$ 


\subsection{1 $\left[\mathrm{NH}_{3} \cdot \mathrm{LiN}\left(\mathrm{SiMe}_{3}\right)_{2}\right]_{2} \cdot \mathrm{TOL}$}

\subsubsection{Cystal Data}

Formula: $\mathrm{C}_{19} \mathrm{H}_{50} \mathrm{Li}_{2} \mathrm{~N}_{4} \mathrm{Si}_{4}, M=460.87 \mathrm{~g} / \mathrm{mol}$, triclinic space group $P \overline{1}, a=9.561(2), b=$ 11.710(2), $c=15.437(3) \AA, \alpha=92.96(2)^{\circ}, \beta=105.26(2)^{\circ}, \gamma=110.88(2)^{\circ}, V=1537.5(6) \AA^{3}$, $\mathrm{Z}=2, \mu\left(\mathrm{MoK}_{\mathrm{a}}\right)=0.205 \mathrm{~mm}^{-1}, 70826$ reflections collected, 6034 independent reflections $\left(R_{\text {int }}=1.88 \%\right), \theta_{\max }=26.02^{\circ}, 293$ parameters refined, 30 restraints used, $R_{1}[I>2 \sigma(I)]=$ $4.64 \%, w R_{2}$ (all data) $=11.87 \%$, GooF $=1.231$, largest diff. peak and hole 0.386 and $-0.322 \mathrm{e}^{-3}$.
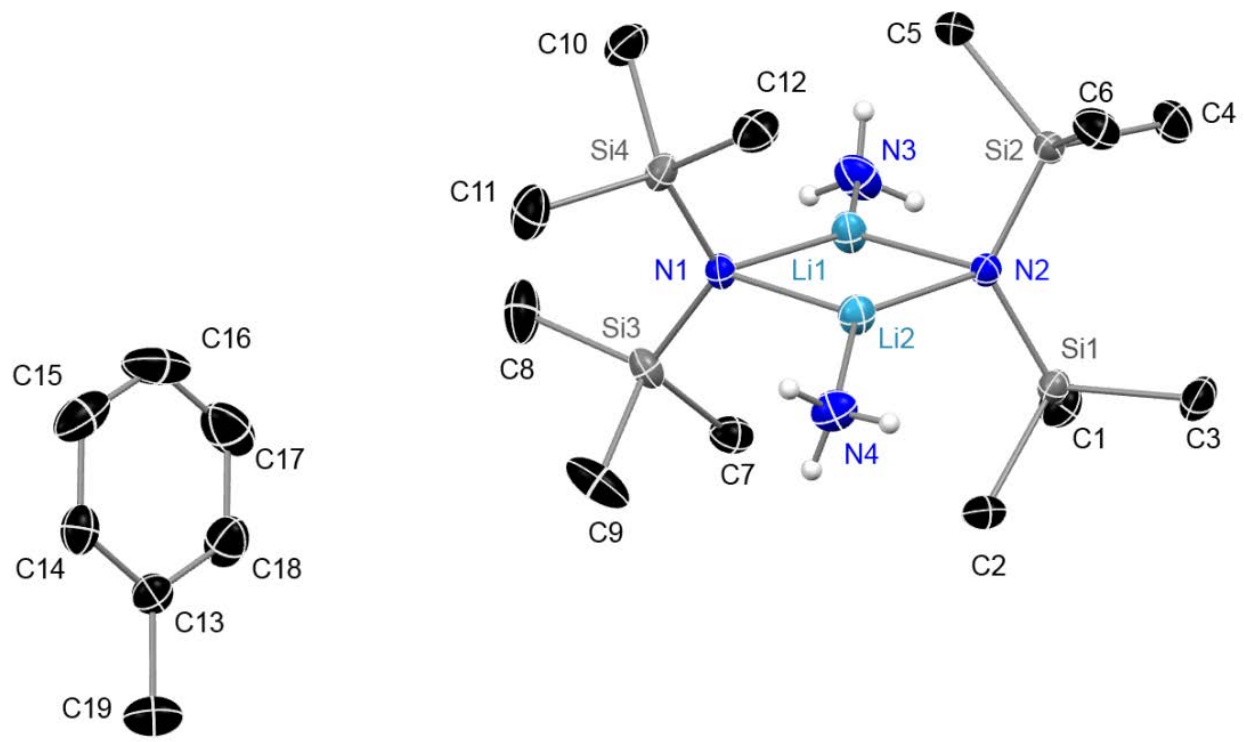

Fig. 4-1. Asymmetric unit of 65.TOL with carbon bound hydrogen atoms omitted for clarity. Anisotropic displacement parameters are depicted at the $50 \%$ probability level.

Selected bond lengths $(\AA)$ and angles $\left({ }^{\circ}\right)$ : N1-Si1 1.696(2), N1-Si2 1.698(2), N1-Li2 2.032(5), N1-Li1 2.044(5), N2-Li1 2.029(5), N2-Si3 1.695(2), N2-Si4 1.697(2), N2-Li2 2.031(5), N3-Li1 2.044(5), N4-Li2 2.046(5); Si1-N1-Si2 123.6(1), Si3-N2-Si4 122.7(1), N2-Li2-N1 105.6(2), N2-Li1-N1 105.2(2), Li2-N1-Li1 74.4(2), Li1-N2-Li2 74.7(2). 


\subsection{2 $\left[\mathrm{NH}_{3} \cdot \mathrm{NaN}\left(\mathrm{SiMe}_{3}\right)_{2}\right]_{2}$}

\subsubsection{Cystal Data}

Formula: $\mathrm{C}_{12} \mathrm{H}_{42} \mathrm{~N}_{4} \mathrm{Na}_{2} \mathrm{Si}_{4}, M=400.83 \mathrm{~g} / \mathrm{mol}$, triclinic space group $P \overline{1}, a=9.459(2), b=$ 10.088(2), $c=14.539(3) \AA ̊ ., \alpha=72.76(2)^{\circ}, \beta=84.69(2)^{\circ}, \gamma=84.69(2)^{\circ}, V=84.69(2)^{\circ} \AA^{3}, Z=$ $2, \mu\left(\mathrm{MoK}_{\alpha}\right)=0.260 \mathrm{~mm}^{-1}, 83314$ reflections collected, 5797 independent reflections $\left(R_{\text {int }}=\right.$ $2.16 \%), \theta_{\max }=27.12^{\circ}, 230$ parameters refined, 30 restraints used, $R_{1}[I>2 \sigma(I)]=3.25 \%$, $w R_{2}($ all data $)=8.51 \%$, GooF $=1.158$, largest diff. peak and hole 0.418 and $-0.301 \mathrm{e}^{-3}$.

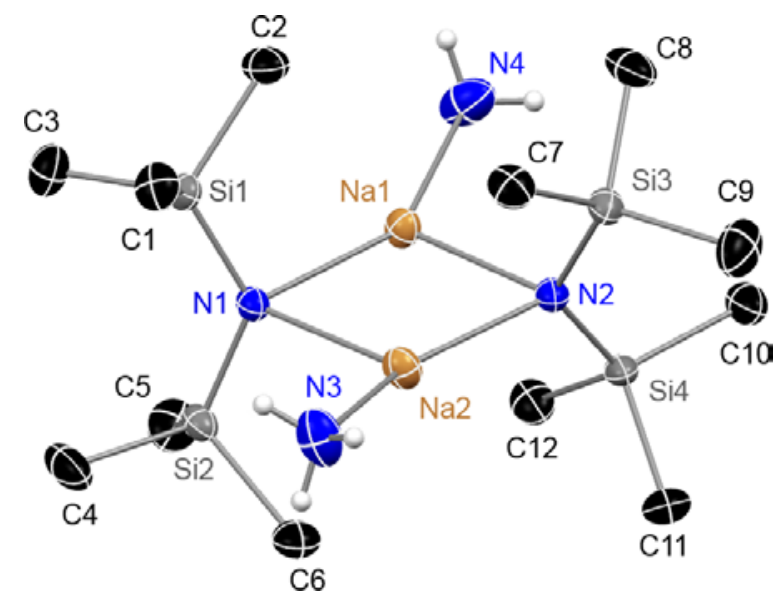

Fig. 4-2. Asymmetric unit of 66 with carbon bound hydrogen atoms omitted for clarity. Anisotropic displacement parameters are depicted at the $50 \%$ probability level.

The structure of $\mathbf{6 6}$ was refined as a twin with BASF $=0.5$. Selected bond lengths $(\AA)$ and angles $\left({ }^{\circ}\right)$ : N1-Si1 1.684(2), N1-Si1 1.684(2), N1-Si2 1.682(2), N1-Na1 2.401(2), N1-Na2 2.396(2), N2-Si3 1.683(2), N2-Si3 1.683(2), N2-Na1 2.407(2), N2-Na2 2.384(2), N3-Na2 2.402(2), N4-Na1 2.420(2); Si2-N1-Si1 127.31(9), Si2-N1-Si1 127.31(9), N1-Na1-N2 100.00(5), N2-Na2-N1 100.82(5), Na2-N1-Na1 79.53(5), Na2-N2-Na1 79.64(5). 


\subsection{3 $\left[\mathrm{NH}_{3} \cdot \mathrm{KN}\left(\mathrm{SiMe}_{3}\right)_{2}\right]_{2}$}

\subsubsection{Cystal Data}

Formula: $\mathrm{C}_{12} \mathrm{H}_{42} \mathrm{~K}_{2} \mathrm{~N}_{4} \mathrm{Si}_{4}, M=433.05 \mathrm{~g} / \mathrm{mol}$, monoclinic space group $P 2_{1} / n, a=9.339(2), b=$

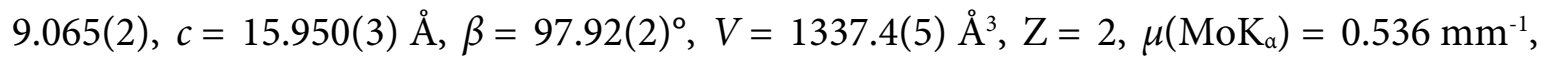
44595 reflections collected, 3059 independent reflections $\left(R_{\text {int }}=3.47 \%\right), \theta_{\max }=27.48^{\circ}, 134$ parameters refined, 127 restraints used, $R_{1}[I>2 \sigma(I)]=2.51 \%, w R_{2}($ all data $)=6.45 \%$, GooF $=$ 1.081, largest diff. peak and hole 0.272 and $-0.191 \mathrm{e}^{-3}$.

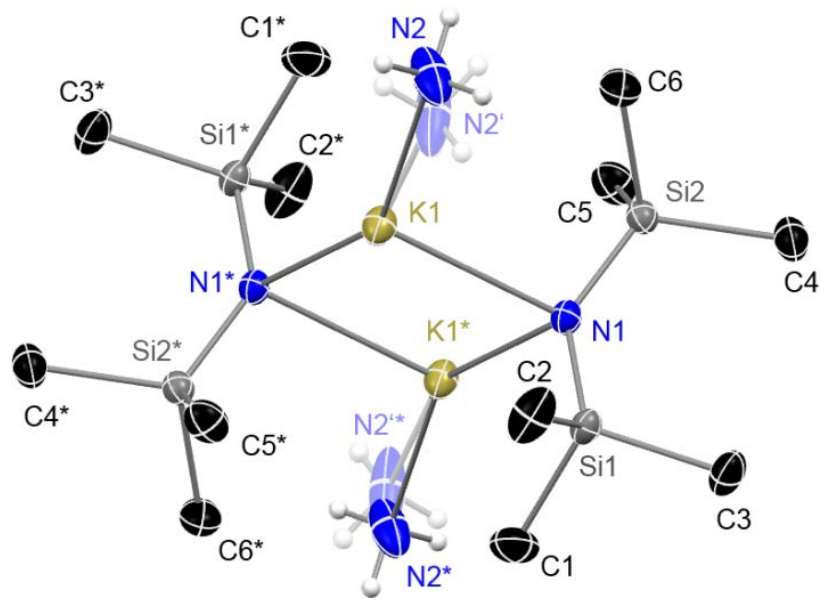

Fig. 4-3. Asymmetric unit of 67 with carbon bound hydrogen atoms omitted for clarity. Anisotropic displacement parameters are depicted at the $50 \%$ probability level.

Disorder of $\mathrm{N} 2$ and N2'was refined with restraints for $\mathrm{K}-\mathrm{N}$ and $\mathrm{H} \cdots \mathrm{K}$ 1,2-distances an occupancy ratio of 0.570:0.430. Selected bond lengths $(\AA)$ and angles $\left(^{\circ}\right)$ : N1-Si1 1.669(1), $\mathrm{N} 1-\mathrm{Si} 2$ 1.669(1), N1-K1 2.792(1), N1-K1* 2.834(1), N2-K1 2.829(6), N2'-K1 2.839(8); $\mathrm{N} 1-\mathrm{K} 1-\mathrm{N} 1 *$ 92.76(3), K1-N1-K1* 87.23(3), Si1-N1-Si2 135.46(7). Atoms highlighted with an asterisk are symmetry generated by $2-\mathrm{x}, 1-\mathrm{y}, 1-\mathrm{z}$. 


\subsection{4 $\left[\mathrm{NH}_{3} \cdot \mathrm{RbN}\left(\mathrm{SiMe}_{3}\right)_{2}\right]_{2}$}

\subsubsection{Cystal Data}

Formula: $\mathrm{C}_{12} \mathrm{H}_{42} \mathrm{~N}_{4} \mathrm{Rb}_{2} \mathrm{Si}_{4}, M=525.79 \mathrm{~g} / \mathrm{mol}$, monoclinic space group $P 2_{1} / c, a=8.825(2)$, $b=12.597(2), \quad c=12.410(3) \AA, \beta=106.88(2)^{\circ}, \quad V=12.410(3) \AA^{3}, Z=2, \mu\left(M_{o}\right)=$ $2.095 \mathrm{~mm}^{-1}, 48496$ reflections collected, 3043 independent reflections $\left(R_{\mathrm{int}}=10.21 \%\right), \theta_{\max }=$ $21.46^{\circ}, 118$ parameters refined, 40 restraints used, $R_{1}[I>2 \sigma(I)]=2.52 \%, w R_{2}$ (all data) $=$ $5.00 \%, \mathrm{GooF}=1.018$, largest diff. peak and hole 0.387 and $-0.394 \mathrm{e}^{-3}$.

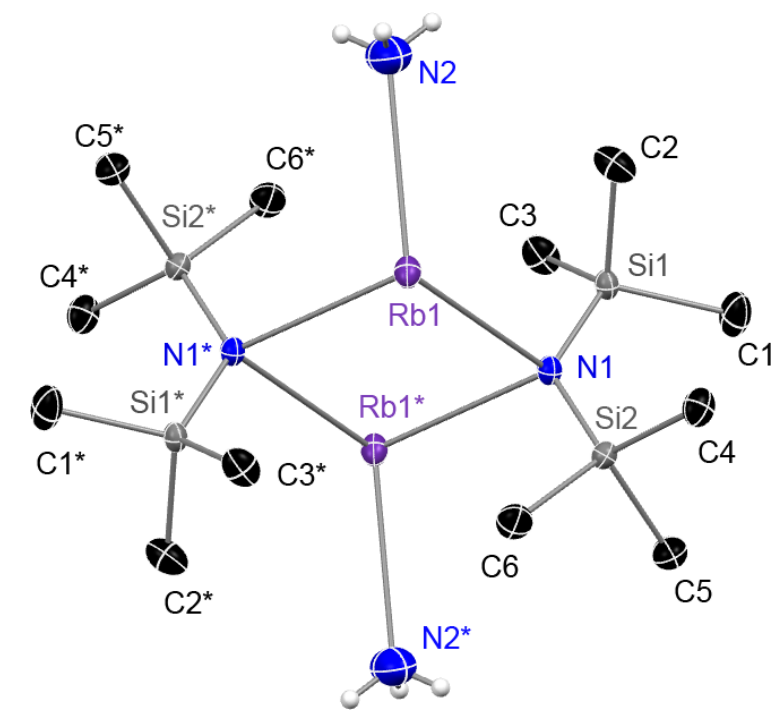

Fig. 4-4. Asymmetric unit of 68 with carbon bound hydrogen atoms omitted for clarity. Anisotropic displacement parameters are depicted at the $50 \%$ probability level.

Selected bond lengths $(\AA)$ and angles $\left(^{\circ}\right)$ : N1-Si1 1.676(2), N1-Si2 1.672(2), N1-Rb1 2.908(2), N1-Rb1* 3.016(2), N2-Rb1 3.014(3); N1-Rb1-N1* 95.83(4), Rb1-N1-Rb1* 84.17(4), Si2-N1-Si1 125.6(1). Atoms highlighted with an asterisk are symmetry generated by $-x, 1-y,-z$. 


\subsection{5 $\left(\mathrm{NH}_{3}\right)_{4} \cdot\left[\mathrm{NaN}\left(\mathrm{SiMe}_{3}\right)_{2}\right]_{2}$}

\subsubsection{Cystal Data}

Formula: $\mathrm{C}_{12} \mathrm{H}_{48} \mathrm{~N}_{6} \mathrm{Na}_{2} \mathrm{Si}_{4}, M=434.90 \mathrm{~g} / \mathrm{mol}$, monoclinic space group $P 2_{1} / n, a=19.146(2)$, $b=9.876(2), \quad c=30.726(3) \AA, \beta=91.82(2)^{\circ}, \quad V=5806.9(14) \AA^{3}, Z=8, \mu\left(M_{\alpha}{ }_{a}\right)=$ $0.242 \mathrm{~mm}^{-1}, 205785$ reflections collected, 11875 independent reflections $\left(R_{\text {int }}=6.21 \%\right)$, $\theta_{\max }=26.37^{\circ}, 529$ parameters refined, 552 restraints used, $R_{1}[I>2 \sigma(I)]=3.31 \%, w R_{2}$ (all data $=8.98 \%, \mathrm{GooF}=1.042$, largest diff. peak and hole 0.510 and $-0.232 \mathrm{e}^{-3}$.

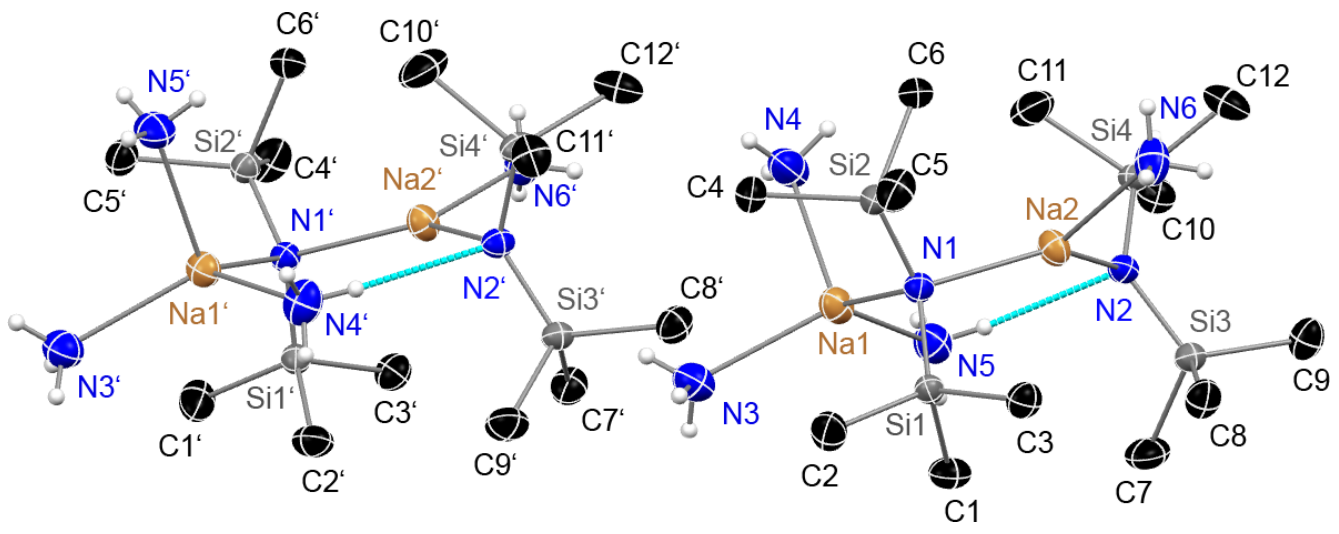

Fig. 4-5. Asymmetric unit of 71 with carbon bound hydrogen atoms omitted for clarity. The asymmetric unit contains two molecules. Anisotropic displacement parameters are depicted at the $50 \%$ probability level.

Selected bond lengths $(\AA)$ and angles $\left({ }^{\circ}\right)$ : N1-Si1 1.689(1), N1-Si2 1.685(1), N1-Na1 2.450(1), N1-Na2 2.387(1), N2-Si3 1.679 (1), N2-Si4 1.670(1), N2-Na2 2.339(1), N3-Na1 2.472(2), N4-Na1 2.469(2), N5-Na1 2.432(2), N6-Na2 2.418 (2), N5-N2 3.219(2), N5-H‥N2 2.39(1); N5-H-N2 172(2), Si2-N1-Si1 125.35(8), Si4-N2-Si3 127.95(8), Na2-N1-Na1 95.34(5), N5-Na1-N1 111.71(5), N2-Na2-N1 137.77(5); N1'-Si1' 1.691(1), N1'-Si2' 1.683(1), N1'-Na1' 2.440(1), N1'-Na2' 2.386(1), N2'-Si3' 1.679(1), N2'-Si4' 1.669(1), N2'-Na2' 2.335 (1), N3'-Na1' 2.469(2), N4'-Na1' 2.437(2), N5'-Na1' 2.477(2),

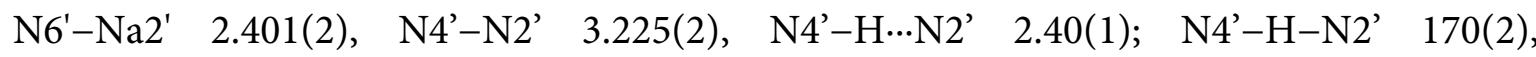
$\mathrm{Si2}-\mathrm{N1}{ }^{\prime}-\mathrm{Si1} 1$ 126.17(8), Si4'-N2'-Si3' 128.59(8), Na2'-N1'-Na1' 95.73(5), N5'-Na1'-N1' 116.92(6), N2'-Na2'-N1' 139.99(5). 


\subsection{6 $\left[(\mathrm{THF}) \mathrm{CIMg}\left(\mu-{ }^{j} \mathrm{Pr}_{2} \mathrm{~N}\right)\right]_{2}$}

\subsubsection{Crystal Data}

Formula: $\mathrm{C}_{20} \mathrm{H}_{44} \mathrm{Cl}_{2} \mathrm{Mg}_{2} \mathrm{~N}_{2} \mathrm{O}_{2}, M=464.09 \mathrm{~g} / \mathrm{mol}$, monoclinic, space group $P 2_{1} / n, a=$ 9.766(2), $b=9.648(2), c=14.138(3) \AA, \beta=110.00(2)^{\circ}, V=1251.8(4) \AA^{3}, Z=2, \mu\left(M_{o K}\right)=$ $0.327 \mathrm{~mm}^{-1}, 33818$ reflections collected, 2666 independent reflections $\left(R_{\text {int }}=3.91 \%\right), \theta_{\max }=$ $26.75^{\circ}, 230$ parameters refined, 686 restraints used, $R_{1}[I>2 \sigma(I)]=3.00 \%, w R_{2}$ (all data) $=$ $7.96 \%$, GooF $=1.067$, largest diff. peak and hole 0.285 and $-0.167 \mathrm{e}^{-3}$. Crystallographic data for compound $[7 \cdot \mathrm{THF}]_{2}$ have been deposited at the Cambridge Crystallographic Data Centre (CCDC) 1423230.

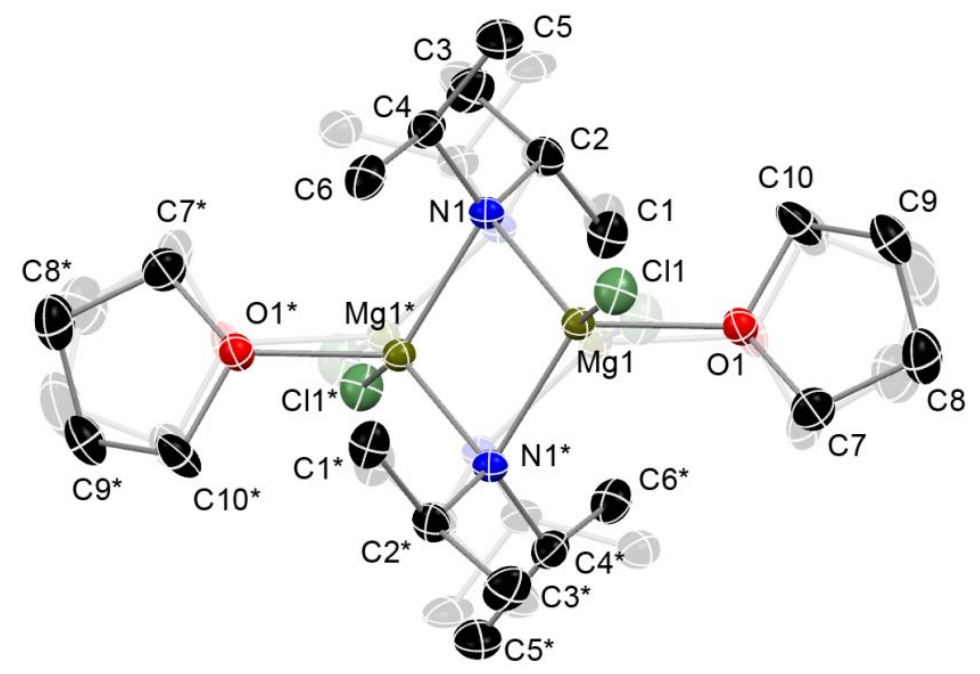

Fig. 4-6. Asymmetric unit of [7.THF $]_{2}$ with hydrogen atoms omitted for clarity. Anisotropic displacement parameters are depicted at the $50 \%$ probability level.

Selected bond lengths $(\AA)$ and angles $\left(^{\circ}\right)$ : Mg1-N1 2.142(1), Mg1-N1* 2.142(3), Mg1-Cl1 2.324(1), Mg1-O1 2.070(6), N1-C2 1.510(3), N1-C4 1.503(3), O1-Mg1-N1 113.5(2), $\mathrm{O} 1-\mathrm{Mg} 1-\mathrm{Cl} 1 \quad 91.8(1), \quad \mathrm{N} 1^{\star}-\mathrm{Mg} 1-\mathrm{N} 1 \quad 96.9(1), \quad \mathrm{Mg} 1^{\star}-\mathrm{N} 1-\mathrm{Mg} 1 \quad$ 83.2(1), $\mathrm{N} 1-\mathrm{Mg} 1-\mathrm{N1}^{*}-\mathrm{Mg} 1^{\prime} 0.0$. Atoms highlighted with an asterisk are symmetry generated by $1-\mathrm{x}, 1-\mathrm{y}, 1-\mathrm{z}$.

The structure is severely disordered and refined with distances restraints and restraints for the anisotropic displacement parameters. ${ }^{[149]}$ However, the disorder was deconvoluted and refined successfully. The occupancy ratio was refined to $0.809: 0.191$. This disorder seems to be an inherent problem with this structure, since García-Álvarez and Mulvey et al. had also problems to crystallize non-disordered crystals of the related $\mathrm{LiCl}$ coordinated compound LiCl.D1·LiCl. ${ }^{[28]}$ 


\section{$5 \quad$ Appendix}

\section{Estimating the Maximum Error of $\log D_{x, \text { norm }}$ in TOL- $d_{8}$ and THF- $d_{8}$}

All measurements were performed at $25^{\circ} \mathrm{C}$. All compounds have been measured in $15 \mathrm{mM}$ solutions of analyte and reference in an equimolar ratio. The absolute diffusion coefficients $\left(D_{\mathrm{x}}\right)$ of all compounds are different on each NMR device. But the normalized diffusion coefficients $\log D_{\mathrm{x}, \text { norm }}$ shows on all devices nearly the same value with a small average standard deviation of $\sigma=0.0028$ in TOL- $d_{8}$ and $\sigma=0.0020$ in THF- $d_{8}$, see A-Table land ATable 2. 
A-Table 1. Diffusion parameter measured on two different NMR-devices (in TOL- $d_{8}$ with ADAM as internal reference).

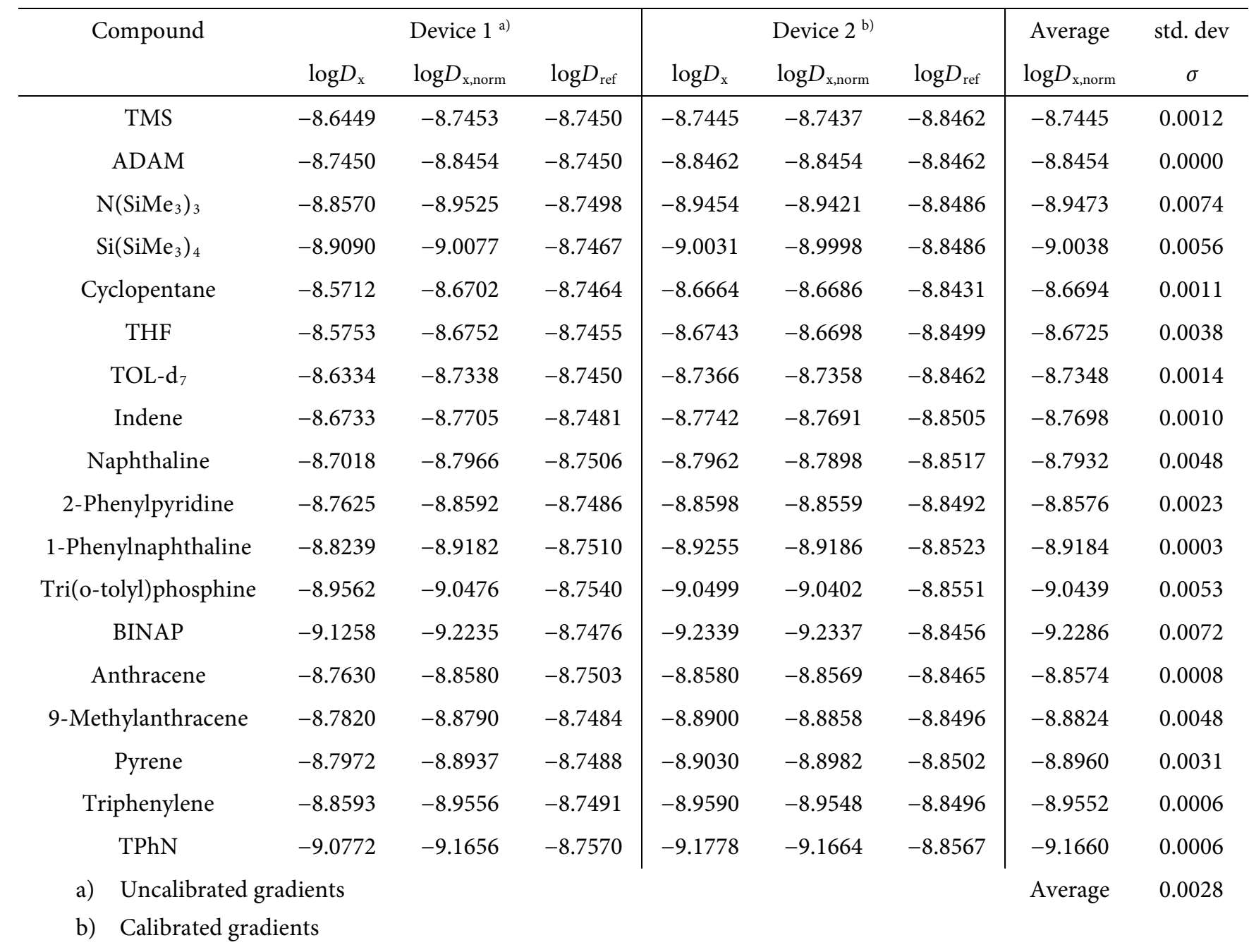


A-Table 2. Diffusion parameter measured on two different NMR-devices (in THF- $d_{8}$ and TMB as internal standard).

\begin{tabular}{|c|c|c|c|c|c|c|c|c|}
\hline \multirow[t]{2}{*}{ Compound } & \multicolumn{3}{|c|}{ Device $1^{\text {a) }}$} & \multicolumn{3}{|c|}{ Device $2^{\mathrm{b})}$} & \multirow{2}{*}{$\begin{array}{l}\text { Average } \\
\log D_{\mathrm{x}, \text { norm }}\end{array}$} & \multirow{2}{*}{$\begin{array}{c}\text { std. dev } \\
\sigma\end{array}$} \\
\hline & $\log D_{\mathrm{x}}$ & $\log D_{\mathrm{x}, \text { norm }}$ & $\log D_{\text {ref }}$ & $\log D_{\mathrm{x}}$ & $\log D_{x, \text { norm }}$ & $\log D_{\text {ref }}$ & & \\
\hline TMS & -8.5969 & -8.6993 & -8.6724 & -8.7018 & -8.7043 & -8.7724 & -8.7018 & 0.0035 \\
\hline TMB & -8.6724 & -8.7749 & -8.6724 & -8.7724 & -8.7749 & -8.7724 & -8.7749 & 0.0000 \\
\hline $\mathrm{N}\left(\mathrm{SiMe}_{3}\right)_{3}$ & -8.8091 & -8.9124 & -8.6716 & -8.8993 & -8.9018 & -8.7724 & -8.9071 & 0.0075 \\
\hline $\mathrm{Si}\left(\mathrm{SiMe}_{3}\right)_{4}$ & -8.8765 & -8.9787 & -8.6726 & -8.9767 & -8.9759 & -8.7757 & -8.9773 & 0.0020 \\
\hline Cyclopentane & -8.5381 & -8.6439 & -8.6690 & -8.6428 & -8.6435 & -8.7742 & -8.6437 & 0.0003 \\
\hline THF- $\mathrm{d}_{7}$ & -8.5303 & -8.6328 & -8.6724 & -8.6368 & -8.6393 & -8.7724 & -8.6360 & 0.0046 \\
\hline Indene & -8.6276 & -8.7326 & -8.6698 & -8.7306 & -8.7323 & -8.7731 & -8.7325 & 0.0002 \\
\hline Naphthaline & -8.6432 & -8.7458 & -8.6722 & -8.7459 & -8.7464 & -8.7744 & -8.7461 & 0.0004 \\
\hline 2-Phenylpyridine & -8.6950 & -8.7996 & -8.6702 & -8.7968 & -8.7980 & -8.7737 & -8.7988 & 0.0011 \\
\hline 1-Phenylnaphthalin & -8.7762 & -8.8799 & -8.6712 & -8.8918 & -8.8812 & -8.7854 & -8.8806 & 0.0009 \\
\hline Tri(o-tolyl)phosphine & -8.8904 & -8.9914 & -8.6739 & -8.9965 & -8.9957 & -8.7757 & -8.9935 & 0.0030 \\
\hline BINAP & -9.0678 & -9.1666 & -8.6761 & -9.1670 & -9.1661 & -8.7757 & -9.1663 & 0.0003 \\
\hline Anthracene & -8.7083 & -8.8140 & -8.6692 & -8.8118 & -8.8117 & -8.7749 & -8.8129 & 0.0016 \\
\hline Pyrene & -8.7453 & -8.8436 & -8.6765 & -8.8503 & -8.8479 & -8.7773 & -8.8457 & 0.0030 \\
\hline Triphenylene & -8.7904 & -8.8889 & -8.6763 & -8.8852 & -8.8849 & -8.7752 & -8.8869 & 0.0028 \\
\hline $\mathrm{TPhN}$ & -9.0082 & -9.1057 & -8.6774 & -9.1103 & -9.1050 & -8.7802 & -9.1054 & 0.0005 \\
\hline & & & & & & & Average & 0.0020 \\
\hline
\end{tabular}

a) Uncalibrated gradients

b) Calibrated gradients 


\section{Overview of the Used Model Compounds for ECCs}

Three dimensional models that were geometry optimized with the program Avogadro 1.1.0 have been generated. Of course the transitions between the shapes are not sharp but there are clear systematic trends that can be rationalized. A-Table 3 displays, that compact spherical (CS) molecules have nearly the same radius in all dimensions with a highly filled space. Dissipated spheres and ellipsoids (DSE) have an elongated main-axis and a less filled space. Small annelated aromatic compounds like toluene $(92 \mathrm{~g} / \mathrm{mol})$, indene $(116 \mathrm{~g} / \mathrm{mol})$ or naphthaline $(128 \mathrm{~g} / \mathrm{mol})$ with $\mathrm{MW}<150 \mathrm{~g} / \mathrm{mol}$ diffuse DSE-like. Also diphenylacetylene $(178 \mathrm{~g} / \mathrm{mol})$ that has an elongated molecule is still in the range of a DSE shape. The significance of one and two dimensional shapes begins approximately at MW $>178 \mathrm{~g} / \mathrm{mol}$. This is why the $\mathrm{ECC}_{\mathrm{ED}}$ for extended discs (ED) begins with anthracene that has a MW of $178 \mathrm{~g} / \mathrm{mol}$.

A-Table 3. Classification of all model compounds appropriate to their shapes.

\begin{tabular}{cccc}
\hline$M W_{\text {calc }}[\mathrm{g} / \mathrm{mol}]$ & $\begin{array}{c}\text { Compact Spheres } \\
{[\mathrm{g} / \mathrm{mol}]}\end{array}$ & $\begin{array}{c}\text { Dissipated Spheres and Ellipsoids } \\
{[\mathrm{g} / \mathrm{mol}]}\end{array}$ & $\begin{array}{c}\text { Expanded Discs } \\
{[\mathrm{g} / \mathrm{mol}]}\end{array}$ \\
\hline
\end{tabular}

70-90

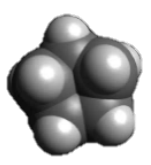

Cyclopentane (70)

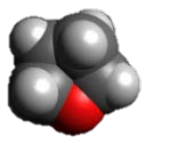

THF (72)

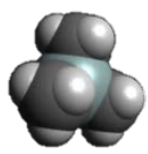

TMS (88)

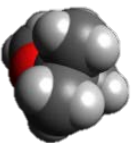

MTBE (88)

100

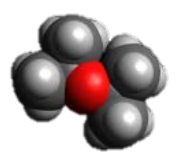

Diisopropylether (102) 


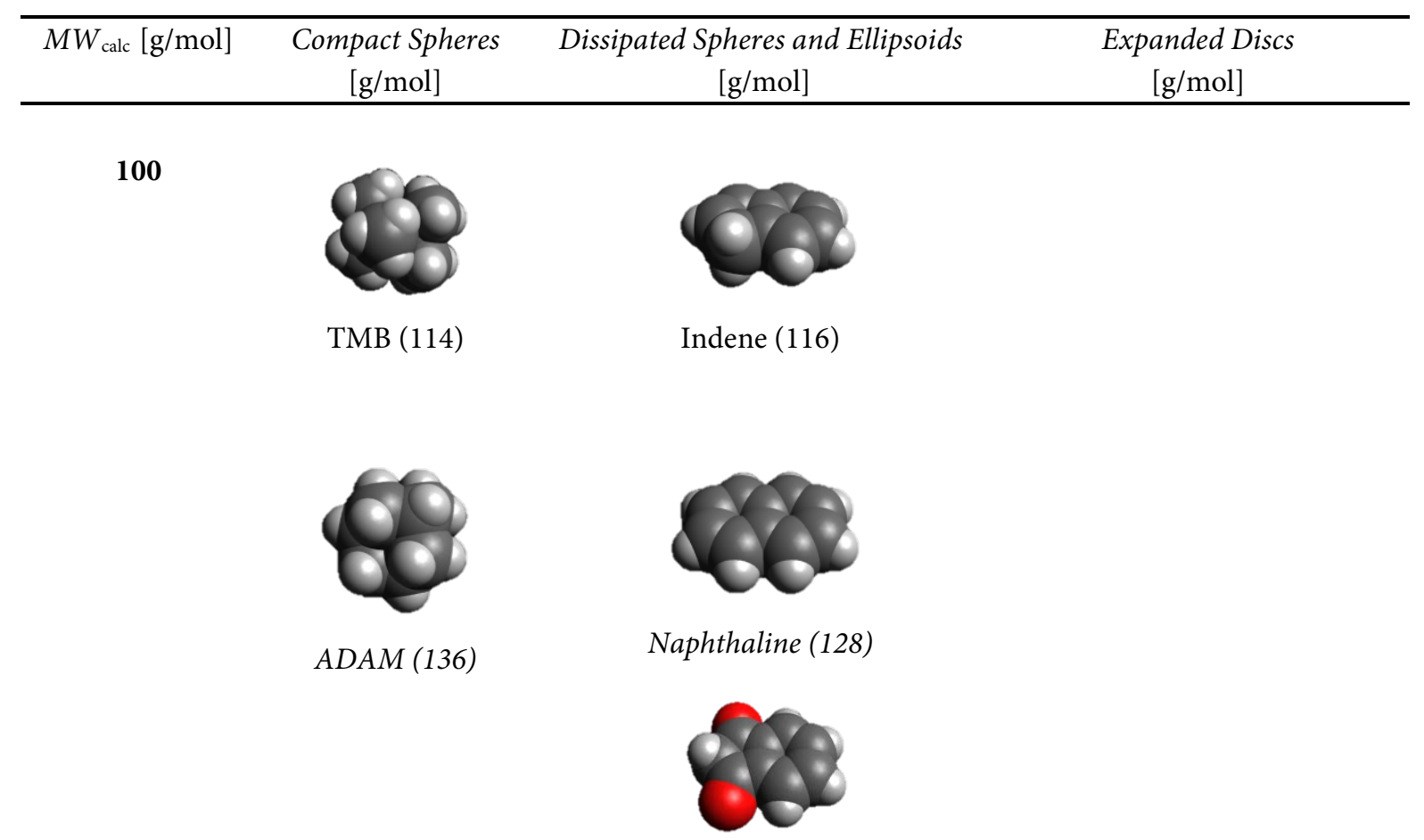

1,3-Indandione (146)

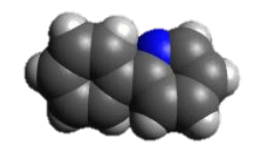

2-Phenylpyridine (155)

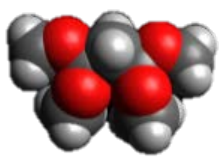

Anthracene (178)

Tetramethoxypropane (164)
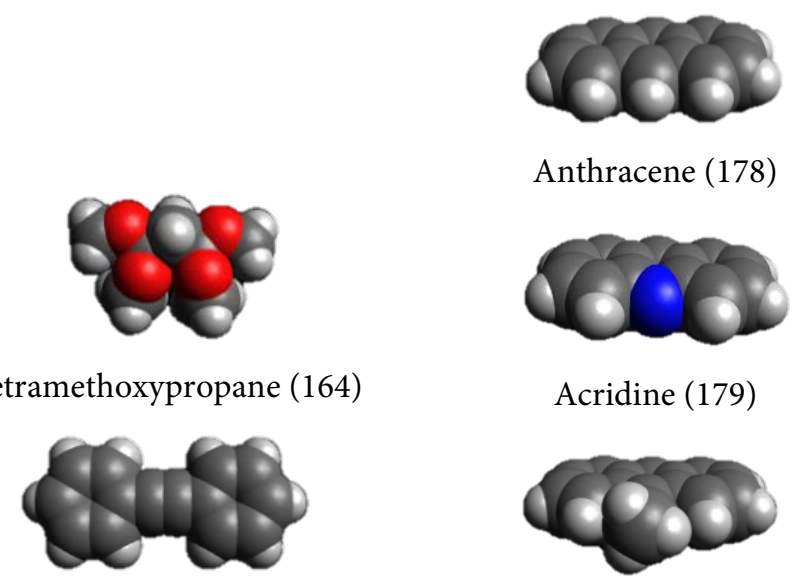

Diphenylacetylene (178)

9-Methylanthracene (192)

200
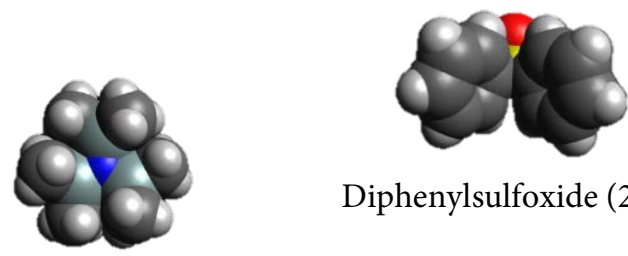

Diphenylsulfoxide (202)

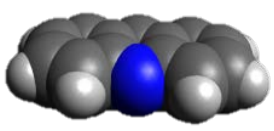

Acridine (179)
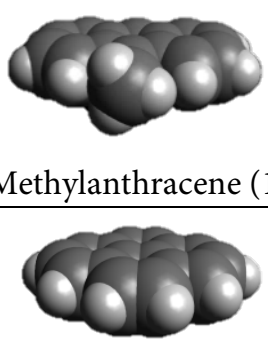

Pyrene (202)

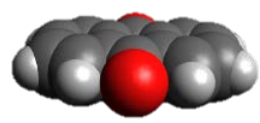

Anthtachinone (208)

$\mathrm{N}\left(\mathrm{SiMe}_{3}\right)_{3}(234)$ 


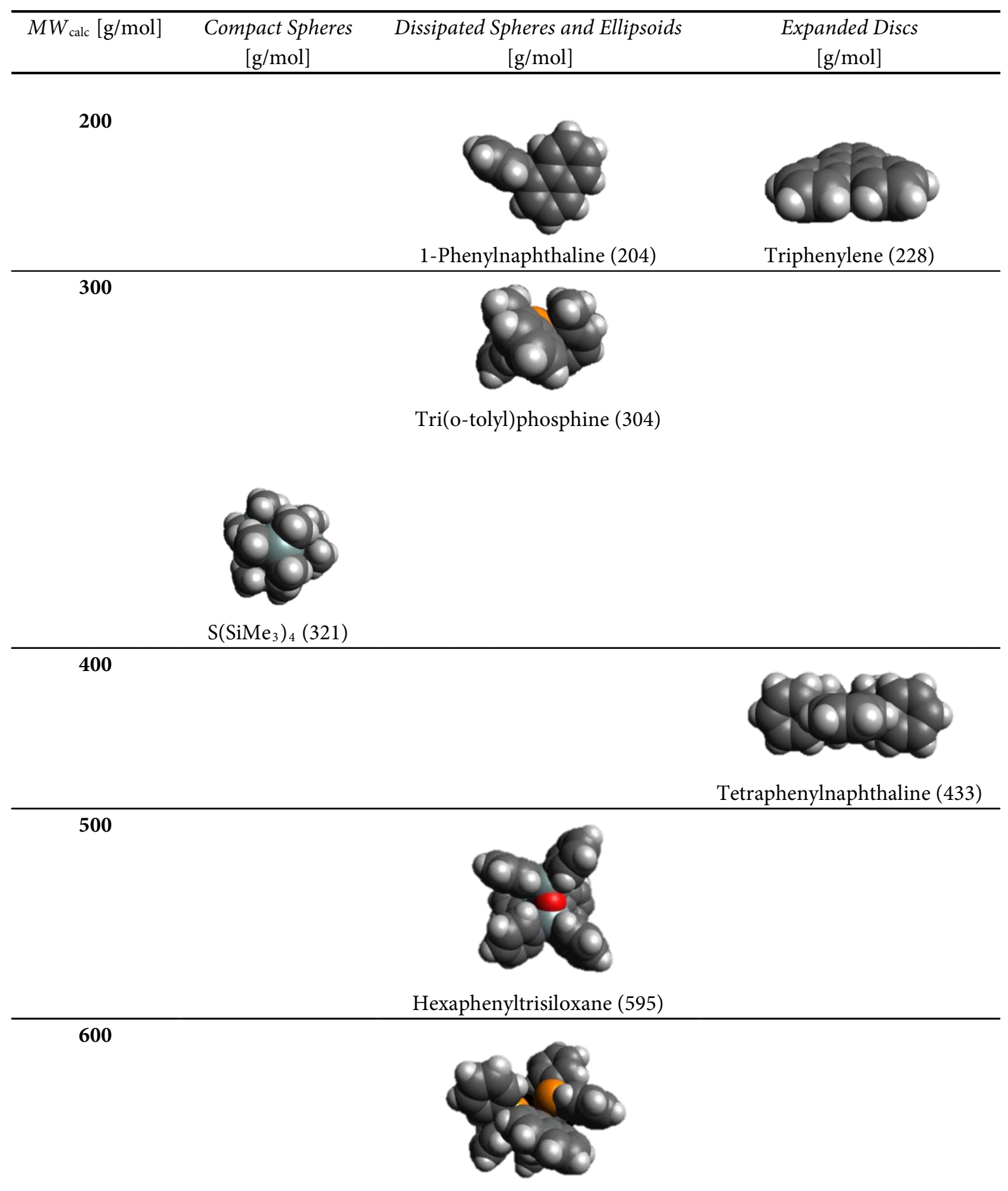

BINAP (623) 


\section{External Calibration Curves}

The maximum deviation of $\log D_{\mathrm{x}, \text { norm }}$ was 0.0075 , which is approximately the width at half maximum of a DOSY signal. This is the reason why the maximum $\Delta \log D_{\mathrm{x}, \text { norm }}$ was defined as $2 \times 0.0075$ which is reflected in the error bars in the calibration plots:
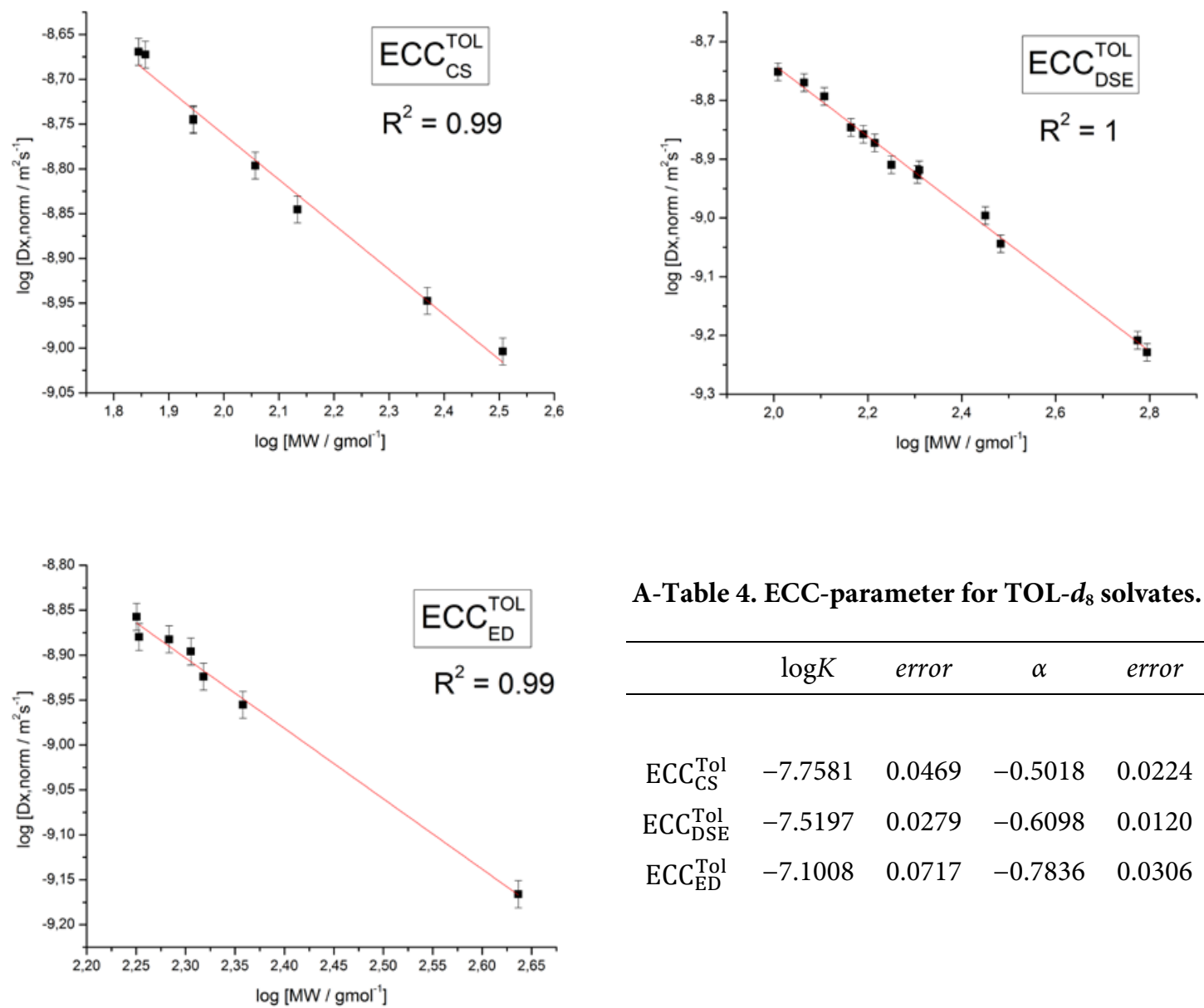

A-Table 4. ECC-parameter for TOL-d 8 solvates.

\begin{tabular}{ccccc}
\hline & $\log K$ & error & $\alpha$ & error \\
\hline & & & & \\
ECC $_{\text {CS }}^{\text {Tol }}$ & -7.7581 & 0.0469 & -0.5018 & 0.0224 \\
ECC $_{\text {DSE }}^{\text {Tol }}$ & -7.5197 & 0.0279 & -0.6098 & 0.0120 \\
ECC $_{\text {ED }}^{\text {Tol }}$ & -7.1008 & 0.0717 & -0.7836 & 0.0306
\end{tabular}

A-Figure 1. Plots of $\log D_{\mathrm{x}, \text { norm }}$ vs $\log M W$ in TOL- $d_{8}$ of all model compounds sorted by their molecular shape. The linear fits show very high accuracy indicated by corr. $\mathrm{R}^{2} \geq 0.99$. 

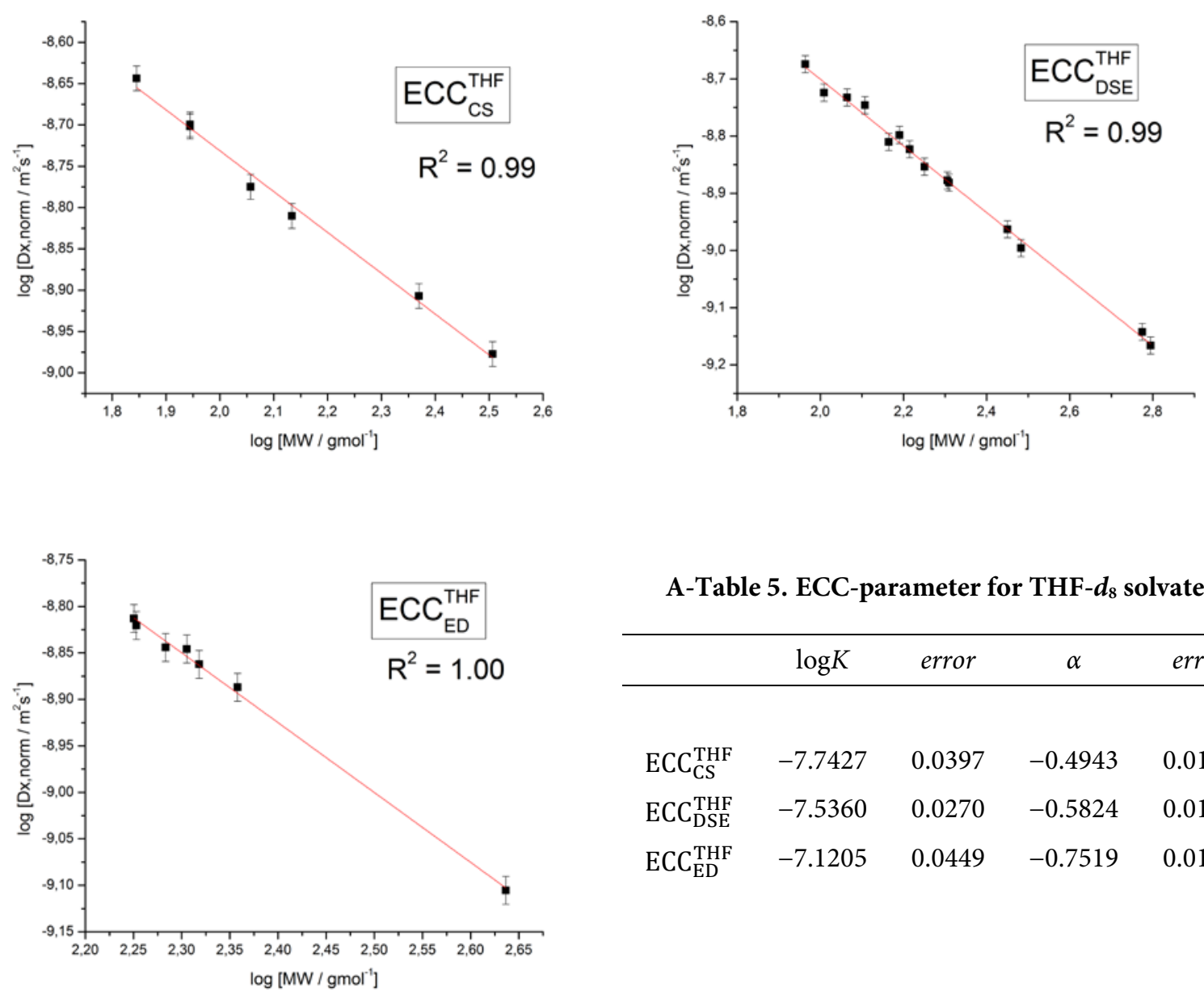

A-Table 5. ECC-parameter for THF- $d_{8}$ solvates

\begin{tabular}{lcccc}
\hline & $\log K$ & error & $\alpha$ & error \\
\hline & & & & \\
ECC $_{\text {CS }}^{\text {THF }}$ & -7.7427 & 0.0397 & -0.4943 & 0.0187 \\
ECC $_{\text {DSE }}^{\text {THF }}$ & -7.5360 & 0.0270 & -0.5824 & 0.0117 \\
ECC $_{\text {ED }}^{\text {THF }}$ & -7.1205 & 0.0449 & -0.7519 & 0.0191
\end{tabular}

A-Figure 2. Plots of $\log D_{\mathrm{x}, \mathrm{norm}} \mathrm{vs} \log M W$ in THF- $d_{8}$ of all model compounds sorted by their molecular shape. The linear fits show very high accuracy indicated by corr. $\mathrm{R}^{2} \geq 0.99$. 
A-Table 6. Overview of the used model compounds for $\mathrm{ECC}^{\mathrm{TO}}$ and their normalized diffusion

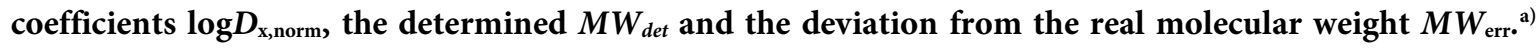
$A D A M$ was used as the internal reference with $\log D_{\text {ref,fix }}(A D A M)=-8.8454$. All compounds have been measured in $15 \mathrm{mM}$ solutions of analyte and ADAM in an equimolar ratio.

\begin{tabular}{|c|c|c|c|c|c|c|}
\hline $\begin{array}{l}M W_{\text {calc }} \\
{[\mathrm{g} / \mathrm{mol}]}\end{array}$ & Compact Spheres, ECC $C S$ & $D_{\mathrm{x}, \text { norm }}\left[\mathrm{m}^{2} / \mathrm{s}\right]$ & $\log D_{\mathrm{x}, \text { norm }}$ & $\log M W_{\text {det }}$ & $\begin{array}{c}M W_{\mathrm{det}} \\
{[\mathrm{g} / \mathrm{mol}]}\end{array}$ & $\begin{array}{c}M W_{\text {err }} \\
{[\%]}\end{array}$ \\
\hline 70 & Cyclopentane & $2.1411 \mathrm{E}-09$ & -8.6694 & 1.8157 & 65 & 7 \\
\hline 72 & $\mathrm{THF}$ & $2.1258 \mathrm{E}-09$ & -8.6725 & 1.8220 & 66 & 8 \\
\hline 88 & TMS & $1.8010 \mathrm{E}-09$ & -8.7445 & 1.9655 & 92 & -5 \\
\hline 88 & MTBE & $1.7965 \mathrm{E}-09$ & -8.7456 & 1.9676 & 93 & -5 \\
\hline 114 & TMB & $1.5985 \mathrm{E}-09$ & -8.7963 & 2.0687 & 117 & -3 \\
\hline 136 & $\mathrm{ADAM}^{\mathrm{b})}$ & $1.4277 \mathrm{E}-09$ & -8.8454 & 2.1665 & 147 & -8 \\
\hline 234 & $\mathrm{~N}\left(\mathrm{SiMe}_{3}\right)_{3}$ & $1.1290 \mathrm{E}-09$ & -8.9473 & 2.3696 & 234 & 0 \\
\hline \multirow[t]{2}{*}{321} & $\mathrm{Si}\left(\mathrm{SiMe}_{3}\right)_{4}$ & $9.9135 \mathrm{E}-10$ & -9.0038 & 2.4821 & 303 & 5 \\
\hline & \multicolumn{2}{|c|}{ Dissipated Spheres \& Ellipsoids, $E C C_{D S E}$} & & & & \\
\hline 102 & Diisopropylether & $1.7727 \mathrm{E}-09$ & -8.7514 & 2.0199 & 105 & -3 \\
\hline 116 & Indene & $1.6990 \mathrm{E}-09$ & -8.7698 & 2.0501 & 112 & 3 \\
\hline 128 & Naphthaline & $1.6099 \mathrm{E}-09$ & -8.7932 & 2.0885 & 123 & 4 \\
\hline 146 & 1,3 Indandione & $1.4257 \mathrm{E}-09$ & -8.8460 & 2.1750 & 150 & -2 \\
\hline 155 & 2-Phenylpyridine & $1.3882 \mathrm{E}-09$ & -8.8576 & 2.1941 & 156 & -1 \\
\hline 164 & Tetramethoxypropane & $1.3420 \mathrm{E}-09$ & -8.8722 & 2.2181 & 165 & -1 \\
\hline 178 & Diphenylacetylene & $1.2316 \mathrm{E}-09$ & -8.9095 & 2.2793 & 190 & -7 \\
\hline 202 & Diphenylsulfoxid & $1.1859 \mathrm{E}-09$ & -8.9260 & 2.3062 & 202 & 0 \\
\hline 204 & 1-Phenylnaphthaline & $1.2067 \mathrm{E}-09$ & -8.9184 & 2.2938 & 197 & 4 \\
\hline 304 & Tri(o-tolyl)phosphine & $9.0389 \mathrm{E}-10$ & -9.0439 & 2.4996 & 316 & -4 \\
\hline 595 & Hexaphenyltrisiloxane & $6.1914 \mathrm{E}-10$ & -9.2082 & 2.7691 & 588 & 1 \\
\hline \multirow[t]{2}{*}{623} & BINAP & $5.9079 \mathrm{E}-10$ & -9.2286 & 2.8025 & 635 & -2 \\
\hline & \multicolumn{2}{|l|}{ Expanded Discs, $E C C_{E D}$} & & & & \\
\hline 178 & Anthracene & $1.3885 \mathrm{E}-09$ & 2.2504 & -8.8574 & 2.2417 & 2 \\
\hline 179 & Acridine & $1.3190 \mathrm{E}-09$ & 2.2529 & -8.8797 & 2.2701 & -4 \\
\hline 192 & 9-Methylanthracene & $1.3110 \mathrm{E}-09$ & 2.2833 & -8.8824 & 2.2735 & 2 \\
\hline 202 & Pyrene & $1.2707 \mathrm{E}-09$ & 2.3054 & -8.8960 & 2.2908 & 3 \\
\hline 208 & Anthrachinone & $1.1915 \mathrm{E}-09$ & 2.3181 & -8.9239 & 2.3265 & -2 \\
\hline 228 & Triphenylene & $1.1087 \mathrm{E}-09$ & 2.3579 & -8.9552 & 2.3664 & -2 \\
\hline \multirow[t]{2}{*}{433} & \multirow[t]{2}{*}{ Tetraphenylnaphthaline } & $6.8229 \mathrm{E}-10$ & 2.6365 & -9.1660 & 2.6355 & 0 \\
\hline & & & & Std. dev. & $\sigma$ & 4 \\
\hline
\end{tabular}

a) When a compound had more than one signal in the ${ }^{1} \mathrm{H}-\mathrm{NMR}$, the average diffusion coefficient was used.

b) For determining the diffusion coefficient, we used the signal of the $-\mathrm{CH}_{2}$ groups with the highest intensity. 
A-Table 7. Overview of the used model compounds for $\mathrm{ECC}^{\mathrm{THF}}$ and their normalized diffusion coefficients $\log D_{\mathrm{x}, \text { norm }}$, the determined $M W_{\text {det }}$ and the deviation $M W_{\text {err. }}$ TMB was used as the internal reference with $\log D_{\text {reffix }}(\mathrm{TMB})=-8.7749$. All compounds have been measured in $15 \mathrm{mM}$ solutions of analyte and TMB in an equimolar ratio. ${ }^{\mathrm{a})}$

\begin{tabular}{|c|c|c|c|c|c|c|}
\hline $\begin{array}{l}M W_{\text {calc }} \\
{[\mathrm{g} / \mathrm{mol}]}\end{array}$ & $\begin{array}{c}\text { Compact Spheres, } \\
E C C_{C S}\end{array}$ & $D_{\mathrm{x}, \mathrm{norm}}\left[\mathrm{m}^{2} / \mathrm{s}\right]$ & $\log D_{\mathrm{x}, \text { norm }}$ & $\log M W_{\text {det }}$ & $\begin{array}{l}M W_{\text {det }} \\
{[\mathrm{g} / \mathrm{mol}]}\end{array}$ & $\begin{array}{c}M W_{\text {err }} \\
{[\%]}\end{array}$ \\
\hline 70 & Cyclopentane & $2.2713 \mathrm{E}-09$ & -8.6437 & 1.8229 & 67 & 5 \\
\hline 88 & TMS & $1.9870 \mathrm{E}-09$ & -8.7018 & 1.9404 & 87 & 1 \\
\hline 88 & MTBE & $1.9980 \mathrm{E}-09$ & -8.6994 & 1.9356 & 86 & 2 \\
\hline 114 & TMB & $1.6793 \mathrm{E}-09$ & -8.7749 & 2.0882 & 123 & -7 \\
\hline 136 & $\mathrm{ADAM}^{\mathrm{b})}$ & $1.5481 \mathrm{E}-09$ & -8.8102 & 2.1597 & 144 & -6 \\
\hline 234 & $\mathrm{~N}\left(\mathrm{SiMe}_{3}\right)_{3}$ & $1.2386 \mathrm{E}-09$ & -8.9071 & 2.3557 & 227 & 3 \\
\hline \multirow[t]{2}{*}{321} & $\mathrm{Si}\left(\mathrm{SiMe}_{3}\right)_{4}$ & $1.0537 \mathrm{E}-09$ & -8.9773 & 2.4977 & 315 & 2 \\
\hline & \multicolumn{2}{|c|}{ Dissipated Spheres \& Ellipsoids, ECC $C_{D S E}$} & & & & \\
\hline 92 & Toluol & $2.1175 \mathrm{E}-09$ & -8.6742 & 1.9543 & 90 & 2 \\
\hline 102 & Diisopropylether & $1.8871 \mathrm{E}-09$ & -8.7242 & 2.0402 & 110 & -8 \\
\hline 116 & Indene & $1.8515 \mathrm{E}-09$ & -8.7325 & 2.0544 & 113 & 2 \\
\hline 128 & Naphthaline & $1.7943 \mathrm{E}-09$ & -8.7461 & 2.0778 & 120 & 7 \\
\hline 146 & 1,3 Indandione & $1.5478 \mathrm{E}-09$ & -8.8103 & 2.1880 & 154 & -6 \\
\hline 155 & 2-Phenylpyridine & $1.5921 \mathrm{E}-09$ & -8.7980 & 2.1670 & 147 & 5 \\
\hline 164 & Tetramethoxypropane & $1.5028 \mathrm{E}-09$ & -8.8231 & 2.2100 & 162 & 1 \\
\hline 178 & Diphenylacetylene & $1.4013 \mathrm{E}-09$ & -8.8535 & 2.2622 & 183 & -3 \\
\hline 202 & Diphenylsulfoxid & $1.3256 \mathrm{E}-09$ & -8.8776 & 2.3035 & 201 & 0 \\
\hline 204 & 1-Phenylnaphthaline & $1.3146 \mathrm{E}-09$ & -8.8812 & 2.3098 & 204 & 0 \\
\hline 304 & Tri(o-tolyl)phosphine & $1.0100 \mathrm{E}-09$ & -8.9957 & 2.5063 & 321 & -6 \\
\hline 595 & Hexaphenyltrisiloxane & $7.2042 \mathrm{E}-10$ & -9.1424 & 2.7583 & 573 & 4 \\
\hline \multirow[t]{2}{*}{623} & BINAP & $6.8215 \mathrm{E}-10$ & -9.1661 & 2.7990 & 629 & -1 \\
\hline & \multicolumn{2}{|l|}{ Expanded Discs, ECC $E D$} & & & & \\
\hline 178 & Anthracene & $1.5386 \mathrm{E}-09$ & -8.8129 & 2.2509 & 178 & 0 \\
\hline 179 & Acridine & $1.5119 \mathrm{E}-09$ & -8.8205 & 2.2610 & 182 & -2 \\
\hline 192 & 9-Methylanthracene & $1.4321 \mathrm{E}-09$ & -8.8440 & 2.2923 & 196 & -2 \\
\hline 202 & Pyrene & $1.4265 \mathrm{E}-09$ & -8.8457 & 2.2946 & 197 & 2 \\
\hline 208 & Anthrachinone & $1.3734 \mathrm{E}-09$ & -8.8622 & 2.3165 & 207 & 0 \\
\hline 228 & Triphenylene & $1.2975 \mathrm{E}-09$ & -8.8869 & 2.3493 & 224 & 2 \\
\hline \multirow[t]{2}{*}{433} & Tetraphenylnaphthaline & $7.8459 \mathrm{E}-10$ & -9.1054 & 2.6399 & 436 & -1 \\
\hline & & & & Std. dev. & $\sigma$ & 4 \\
\hline
\end{tabular}

a) When a compound had more than one signal in the ${ }^{1} \mathrm{H}-\mathrm{NMR}$, the average diffusion coefficient was used.

b) For determining the diffusion coefficient, we used the signal of the $-\mathrm{CH}_{2}$ groups with the highest intensity. 


\section{Influence of High Concentration}

A-Table 8. $\mathrm{ECC}^{\mathrm{TOL}}$ from $15 \mathrm{mM}$ solutions were used to determine the $\mathrm{MW}$ of compounds that were measured in concentrated TOL- $d_{8}$ solutions $(120 \mathrm{mM})$. The deviation $M W_{\text {err }}$ is a little higher than in the dilute solutions but still in a good range. ADAM was used as the internal reference with $\log D_{\text {ref,fix }}$ $(\mathrm{ADAM})=-8.8454 .^{\mathrm{a})}$

\begin{tabular}{|c|c|c|c|c|c|c|}
\hline $\begin{array}{l}M W_{\text {calc }} \\
{[\mathrm{g} / \mathrm{mol}]}\end{array}$ & $\begin{array}{c}\text { Compact Spheres, } \\
E C C_{C S}\end{array}$ & $D_{\mathrm{x}, \text { norm }}\left[\mathrm{m}^{2} / \mathrm{s}\right]$ & $\log D_{\mathrm{x}, \text { norm }}$ & $\log M W_{\text {det }}$ & $\begin{array}{l}M W_{\mathrm{det}} \\
{[\mathrm{g} / \mathrm{mol}]}\end{array}$ & $\begin{array}{c}M W_{\text {err }} \\
{[\%]}\end{array}$ \\
\hline 70 & Cyclopentane & $2.0900 \mathrm{E}-09$ & -8.6799 & 1.8367 & 69 & 2 \\
\hline 72 & THF & $2.0805 \mathrm{E}-09$ & -8.6818 & 1.8406 & 69 & 4 \\
\hline 88 & MTBE & $1.7751 \mathrm{E}-09$ & -8.7508 & 1.9780 & 95 & -8 \\
\hline 88 & TMS & $1.8331 \mathrm{E}-09$ & -8.7368 & 1.9502 & 89 & -1 \\
\hline 136 & $\mathrm{ADAM}^{\mathrm{b})}$ & $1.4277 \mathrm{E}-09$ & -8.8454 & 2.1665 & 147 & -8 \\
\hline 234 & $\mathrm{~N}\left(\mathrm{SiMe}_{3}\right)_{3}$ & $1.1410 \mathrm{E}-09$ & -8.9427 & 2.3605 & 229 & 2 \\
\hline \multirow[t]{2}{*}{321} & $\mathrm{Si}\left(\mathrm{SiMe}_{3}\right)_{4}$ & $9.5908 \mathrm{E}-10$ & -9.0181 & 2.5108 & 324 & -1 \\
\hline & \multicolumn{2}{|c|}{ Dissipated Spheres \& Ellipsoids, ECC $C_{D S E}$} & & & & \\
\hline 102 & Diisopropylether & $1.7278 \mathrm{E}-09$ & -8.7625 & 2.0382 & 109 & -7 \\
\hline 116 & Indene & $1.6664 \mathrm{E}-09$ & -8.7782 & 2.0639 & 116 & 0 \\
\hline 146 & Indandione & $1.3971 \mathrm{E}-09$ & -8.8548 & 2.1895 & 155 & -6 \\
\hline 155 & 2-Phenylpyridine & $1.4019 \mathrm{E}-09$ & -8.8533 & 2.1871 & 154 & 1 \\
\hline 161 & HMDS & $1.3379 \mathrm{E}-09$ & -8.8736 & 2.2203 & 166 & -3 \\
\hline 164 & tetramethoxythane & $1.3153 \mathrm{E}-09$ & -8.8810 & 2.2324 & 171 & -4 \\
\hline 178 & Diphenylacetylene & $1.2020 \mathrm{E}-09$ & -8.9201 & 2.2966 & 198 & -11 \\
\hline 204 & $\mathrm{PhN}$ & $1.1174 \mathrm{E}-09$ & -8.9518 & 2.3486 & 223 & -9 \\
\hline 304 & Tri(o-tolyl)phosphine & $8.4481 \mathrm{E}-10$ & -9.0732 & 2.5477 & 353 & -16 \\
\hline \multirow[t]{2}{*}{595} & Hexaphenyltrisiloxane & $6.0717 \mathrm{E}-10$ & -9.2167 & 2.7830 & 607 & -2 \\
\hline & Expanded Discs, $E C C_{E D}$ & & & & & \\
\hline 192 & 9-Methylanthracene & $1.2259 \mathrm{E}-09$ & -8.9116 & 2.3107 & 205 & -7 \\
\hline 202 & Pyrene & $1.2195 \mathrm{E}-09$ & -8.9138 & 2.3136 & 206 & -2 \\
\hline 228 & Triphenylene & $1.1129 \mathrm{E}-09$ & -8.9535 & 2.3643 & 231 & -1 \\
\hline \multirow[t]{2}{*}{433} & Tetraphenylnaphthaline & $6.2746 \mathrm{E}-10$ & -9.2024 & 2.6819 & 481 & -11 \\
\hline & & & & Std. dev. & $\sigma$ & 5 \\
\hline
\end{tabular}

a) When a compound had more than one signal in the ${ }^{1} \mathrm{H}-\mathrm{NMR}$, the average diffusion coefficient was used.

b) For determining the diffusion coefficient, we used the signal of the $-\mathrm{CH}_{2}$ groups with the highest intensity. 


\section{Testing the Influence of the Temperature on ECCs}

A-Table 9. ECC-MW-determination of $\mathrm{Si}\left(\mathrm{SiMe}_{3}\right)_{4}(321 \mathrm{~g} / \mathrm{mol})$ in TOL- $d_{8}$ and in THF- $d_{8}$. ADAM was used as internal reference in $15 \mathrm{mM}$ solutions.

\begin{tabular}{|c|c|c|c|c|c|}
\hline $\begin{array}{c}\text { Temp } \\
{\left[{ }^{\circ} \mathrm{C}\right]}\end{array}$ & $\begin{array}{l}D_{\mathrm{x}, \mathrm{norm}} \\
{\left[\mathrm{m}^{2} / \mathrm{s}\right]} \\
\end{array}$ & $\log D_{\mathrm{x}, \text { norm }}$ & $\log M W_{\text {det }}$ & $\begin{array}{c}M W_{\text {det }} \\
{[\mathrm{g} / \mathrm{mol}]}\end{array}$ & $\begin{array}{c}M W_{\text {err }} \\
{[\%]}\end{array}$ \\
\hline 100 & $9.5854 \mathrm{E}-10$ & -9.0184 & 2.5113 & 325 & -1 \\
\hline 75 & $9.6025 \mathrm{E}-10$ & -9.0176 & 2.5097 & 323 & -1 \\
\hline 50 & $9.6687 \mathrm{E}-10$ & -9.0146 & 2.5038 & 319 & 1 \\
\hline 25 & $9.5261 \mathrm{E}-10$ & -9.0211 & 2.5166 & 329 & -2 \\
\hline 0 & $9.7043 \mathrm{E}-10$ & -9.0130 & 2.5006 & 317 & 1 \\
\hline-25 & $9.5640 \mathrm{E}-10$ & -9.0194 & 2.5132 & 326 & -2 \\
\hline-50 & $9.9297 \mathrm{E}-10$ & -9.0031 & 2.4807 & 302 & 6 \\
\hline-75 & $9.8113 \mathrm{E}-10$ & -9.0083 & 2.4911 & 310 & 3 \\
\hline \multicolumn{6}{|l|}{$\mathrm{THF}-d_{8}$} \\
\hline $\begin{array}{l}\text { Temp } \\
{\left[{ }^{\circ} \mathrm{C}\right]}\end{array}$ & $\begin{array}{l}D_{\mathrm{x}, \text { norm }} \\
{\left[\mathrm{m}^{2} / \mathrm{s}\right]} \\
\end{array}$ & $\log D_{\mathrm{x}, \text { norm }}$ & $\log M W_{\text {det }}$ & $\begin{array}{c}M W_{\text {det }} \\
{[\mathrm{g} / \mathrm{mol}]}\end{array}$ & $\begin{array}{c}M W_{\text {err }} \\
{[\%]}\end{array}$ \\
\hline 60 & $1.0540 \mathrm{E}-09$ & -8.9772 & 2.4975 & 314 & 2 \\
\hline 45 & $1.0433 \mathrm{E}-09$ & -8.9816 & 2.5065 & 321 & 0 \\
\hline 25 & $1.0671 \mathrm{E}-09$ & -8.9718 & 2.4866 & 307 & 4 \\
\hline 0 & $1.0594 \mathrm{E}-09$ & -8.9749 & 2.4930 & 311 & 3 \\
\hline-25 & $1.0766 \mathrm{E}-09$ & -8.9680 & 2.4788 & 301 & 6 \\
\hline-50 & $1.0331 \mathrm{E}-09$ & -8.9859 & 2.5151 & 327 & -2 \\
\hline-75 & $1.0710 \mathrm{E}-09$ & -8.9702 & 2.4835 & 304 & 5 \\
\hline
\end{tabular}


Calculation of the Molar Van der Waals Density $M D_{\mathrm{w}}$

A-Table 10. Calculation of the molar van der Waals density $M D_{W}$ using equation (25).

\begin{tabular}{|c|c|c|c|c|}
\hline Compound & Formula & $\begin{array}{l}M W_{\text {calc }} \\
{[\mathrm{g} / \mathrm{mol}]}\end{array}$ & $\begin{array}{c}M D_{\mathrm{W}} \\
{\left[\mathrm{g} /\left(\mathrm{mol} \cdot \mathrm{m}^{3}\right)\right]}\end{array}$ & $\begin{array}{l}\Sigma V_{\mathrm{W}} \\
{\left[\mathrm{m}^{3}\right]}\end{array}$ \\
\hline Cyclopentane & $\mathrm{C} 5 \mathrm{H} 10$ & 70 & $4.41 \mathrm{E}+29$ & $1.59 \mathrm{E}-28$ \\
\hline THF & $\mathrm{C} 4 \mathrm{H} 8 \mathrm{O}$ & 72 & $5.08 \mathrm{E}+29$ & $1.42 \mathrm{E}-28$ \\
\hline TMS & $\mathrm{C} 4 \mathrm{H} 12 \mathrm{Si}$ & 88 & $4.68 \mathrm{E}+29$ & $1.88 \mathrm{E}-28$ \\
\hline MTBE & $\mathrm{C} 5 \mathrm{H} 12 \mathrm{O}$ & 88 & $4.77 \mathrm{E}+29$ & $1.85 \mathrm{E}-28$ \\
\hline TMB & $\mathrm{C} 8 \mathrm{H} 18$ & 114 & $4.30 \mathrm{E}+29$ & $2.65 \mathrm{E}-28$ \\
\hline ADAM & $\mathrm{C} 10 \mathrm{H} 16$ & 136 & $4.61 \mathrm{E}+29$ & $2.95 \mathrm{E}-28$ \\
\hline $\mathrm{N}\left(\mathrm{SiMe}_{3}\right)_{3}$ & $\mathrm{C} 9 \mathrm{H} 27 \mathrm{NSi} 3$ & 234 & $5.00 \mathrm{E}+29$ & $4.68 \mathrm{E}-28$ \\
\hline $\mathrm{Si}\left(\mathrm{SiMe}_{3}\right)_{4}$ & $\mathrm{C} 12 \mathrm{H} 36 \mathrm{Si} 5$ & 321 & $5.00 \mathrm{E}+29$ & $6.42 \mathrm{E}-28$ \\
\hline Toluol & $\mathrm{C} 7 \mathrm{H} 8$ & 92 & $4.88 \mathrm{E}+29$ & $1.89 \mathrm{E}-28$ \\
\hline Diisopropylether & $\mathrm{C} 6 \mathrm{H} 14 \mathrm{O}$ & 102 & $4.72 \mathrm{E}+29$ & $2.16 \mathrm{E}-28$ \\
\hline Indene & $\mathrm{C} 9 \mathrm{H} 8$ & 116 & $5.05 \mathrm{E}+29$ & $2.30 \mathrm{E}-28$ \\
\hline Naphthaline & $\mathrm{C} 10 \mathrm{H} 8$ & 128 & $5.11 \mathrm{E}+29$ & $2.50 \mathrm{E}-28$ \\
\hline 1,3 Indandione & $\mathrm{C} 9 \mathrm{H} 6 \mathrm{O} 2$ & 146 & $5.89 E+29$ & $2.48 \mathrm{E}-28$ \\
\hline 2-Phenylpyridine & C11H9N & 155 & $5.31 \mathrm{E}+29$ & $2.92 \mathrm{E}-28$ \\
\hline Tetramethoxypropane & $\mathrm{C} 7 \mathrm{H} 16 \mathrm{O} 4$ & 164 & $5.61 \mathrm{E}+29$ & $2.92 \mathrm{E}-28$ \\
\hline Diphenylacetylene & $\mathrm{C} 14 \mathrm{H} 10$ & 178 & $5.18 \mathrm{E}+29$ & $3.44 \mathrm{E}-28$ \\
\hline Diphenylsulfoxid & $\mathrm{C} 12 \mathrm{H} 10 \mathrm{OS}$ & 202 & $5.91 \mathrm{E}+29$ & $3.42 \mathrm{E}-28$ \\
\hline 1-Phenylnaphthaline & $\mathrm{C} 16 \mathrm{H} 12$ & 204 & $5.15 \mathrm{E}+29$ & $3.96 \mathrm{E}-28$ \\
\hline Tri(o-tolyl)phosphine & $\mathrm{C} 21 \mathrm{H} 21 \mathrm{P}$ & 304 & $5.30 \mathrm{E}+29$ & $5.74 \mathrm{E}-28$ \\
\hline Hexaphenyltrisiloxane & $\mathrm{C} 36 \mathrm{H} 30 \mathrm{O} 3 \mathrm{Si} 3$ & 595 & $5.57 \mathrm{E}+29$ & $1.07 \mathrm{E}-27$ \\
\hline BINAP & $\mathrm{C} 44 \mathrm{H} 32 \mathrm{P} 2$ & 623 & $5.50 \mathrm{E}+29$ & $1.13 \mathrm{E}-27$ \\
\hline Anthracene & $\mathrm{C} 14 \mathrm{H} 10$ & 178 & $5.18 \mathrm{E}+29$ & $3.44 \mathrm{E}-28$ \\
\hline Acridine & $\mathrm{C} 13 \mathrm{H} 9 \mathrm{~N}$ & 179 & $5.37 \mathrm{E}+29$ & $3.33 \mathrm{E}-28$ \\
\hline 9-Methylanthracene & $\mathrm{C} 15 \mathrm{H} 12$ & 192 & $5.11 \mathrm{E}+29$ & $3.76 \mathrm{E}-28$ \\
\hline Pyrene & $\mathrm{C} 16 \mathrm{H} 10$ & 202 & $5.25 \mathrm{E}+29$ & $3.85 \mathrm{E}-28$ \\
\hline Anthrachinone & $\mathrm{C} 14 \mathrm{H} 8 \mathrm{O} 2$ & 208 & $5.74 \mathrm{E}+29$ & $3.62 \mathrm{E}-28$ \\
\hline Triphenylene & $\mathrm{C} 18 \mathrm{H} 12$ & 228 & $5.21 \mathrm{E}+29$ & $4.37 \mathrm{E}-28$ \\
\hline Tetraphenylnaphthaline & $\mathrm{C} 34 \mathrm{H} 24$ & 433 & $5.19 E+29$ & $8.34 \mathrm{E}-28$ \\
\hline 1-Hexylchloride & $\mathrm{C} 6 \mathrm{H} 13 \mathrm{Cl}$ & 120 & $5.49 \mathrm{E}+29$ & $2.18 \mathrm{E}-28$ \\
\hline 1-Octylchloride & $\mathrm{C} 8 \mathrm{H} 17 \mathrm{Cl}$ & 149 & $5.29 \mathrm{E}+29$ & $2.82 \mathrm{E}-28$ \\
\hline 1-Decylchloride & $\mathrm{C} 10 \mathrm{H} 21 \mathrm{Cl}$ & 177 & $5.13 E+29$ & $3.45 \mathrm{E}-28$ \\
\hline 1-Propylbromide & $\mathrm{C} 3 \mathrm{H} 7 \mathrm{Br}$ & 123 & $9.66 \mathrm{E}+29$ & $1.27 \mathrm{E}-28$ \\
\hline Dibromoanthracene & $\mathrm{C} 14 \mathrm{H} 8 \mathrm{Br} 2$ & 336 & $8.71 \mathrm{E}+29$ & $3.86 \mathrm{E}-28$ \\
\hline Triphenylmethylbromid & $\mathrm{C} 19 \mathrm{H} 15 \mathrm{Br}$ & 323 & $6.45 \mathrm{E}+29$ & $5.01 \mathrm{E}-28$ \\
\hline
\end{tabular}




\section{DOSY-Spectrum of LDA in TOL- $d_{8}$ at $-50^{\circ} \mathrm{C}$}

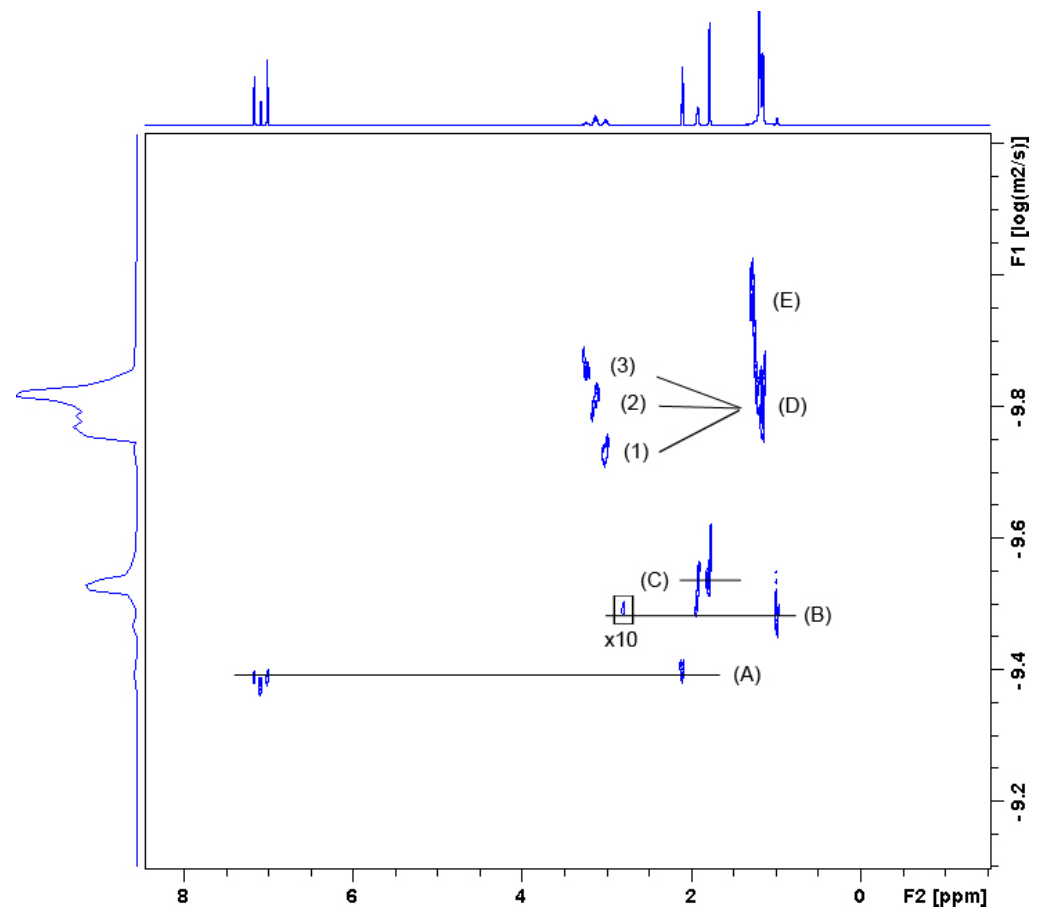

A-Figure 3. DOSY-Spectrum of LDA in TOL- $d_{8}$ at $-50^{\circ} \mathrm{C}$.

A-Table 11. DOSY-ECC-MW-determination of LDA in TOL- $d_{8}$ at $-50^{\circ} \mathrm{C}$.

\begin{tabular}{|c|c|c|c|c|c|c|}
\hline Entry & $\begin{array}{l}\text { Compound } \\
\qquad(\mathrm{g} / \mathrm{mol})\end{array}$ & $\begin{array}{l}D / 10^{-10} \\
{\left[\mathrm{~m}^{2} / \mathrm{s}\right]}\end{array}$ & $\log D_{\mathrm{x}}$ & $\log D_{\mathrm{x}, \mathrm{norm}}$ & $\begin{array}{l}M W_{\operatorname{det}}{ }^{\mathrm{a})} \\
{[\mathrm{g} / \mathrm{mol}]}\end{array}$ & $\begin{array}{c}M W_{\text {err }} \\
{[\%]}\end{array}$ \\
\hline (A) & TOL- $d_{8}(92)^{\mathrm{b}}$ & 3.723 & -9.4291 & -8.6992 & 86 & 7 \\
\hline (B) & Diisopropylamine (101) & 3.402 & -9.4683 & -8.7383 & 100 & 1 \\
\hline (C) & $\operatorname{ADAM}(136)^{c)}$ & 2.659 & -9.5753 & -8.8454 & 147 & -8 \\
\hline (D) & Mix of (1), (2), (3) & & & & & \\
\hline (1) & Trimer (321) & 1.632 & -9.7873 & -9.0574 & 332 & -3 \\
\hline (2) & Tetramer (428) & 1.409 & -9.8511 & -9.1212 & 423 & 1 \\
\hline (3) & Pentamer (536) & 1.243 & -9.9055 & -9.1756 & 520 & 3 \\
\hline (E) & Higher oligomers & & & & & \\
\hline
\end{tabular}

a) All MWs were calculated by using ECC $_{\text {DSE }}$.

b) Deuterated species diffuse like their protonated counterparts.

c) The MW of ADAM, a highly spherical and compact molecule, was calculated by using ECC Cs. $_{\text {. }}$ 
A-Table 12. Relative proton intensities of the $\alpha-\mathrm{CH}$ signals of LDA in TOL- $d_{8}$ at various temperatures. ${ }^{\text {a) }}$

\begin{tabular}{ccccc}
\hline $\begin{array}{c}\text { Temp } \\
{\left[{ }^{\circ} \mathrm{C}\right]}\end{array}$ & $\begin{array}{c}\text { Trimer } \\
A\end{array}$ & $\begin{array}{c}\text { Tetramer } \\
B\end{array}$ & $\begin{array}{c}\text { Pentamer } \\
C\end{array}$ & $\begin{array}{c}\text { Oligomer } \\
D\end{array}$ \\
\hline-75 & 4 & 8 & 5 & 5 \\
-50 & 5 & 8 & 5 & 4 \\
-25 & 6 & 8 & 4 & 4 \\
0 & 10 & 8 & 2 & 2 \\
+25 & 12 & 8 & & $4^{\mathrm{b})}$ \\
+50 & 12 & 8 & & $3^{\mathrm{b})}$ \\
+75 & 28 & 8 & $2^{\mathrm{b})}$ \\
+100 & \multicolumn{5}{|c}{ One coalesced signal }
\end{tabular}

a) Sum of the $\mathrm{a}-\mathrm{CH}$ protons: trimer $(6 \mathrm{H})$, tetramer $(8 \mathrm{H})$, pentamer $(10 \mathrm{H})$. Due to signal overlap the sum of the integrals is not always constant.

b) Pentamer and higher oligomers are not separated.

\section{${ }^{1} \mathrm{H}$-DOSY-ECC-MW-determination parameters of MHMDS ( $\mathrm{M}=\mathrm{Li}, \mathrm{Na}$ and $\mathrm{K}$ ) in TOL- $d_{8}$ with ammonia as donor base}

A-Table 13. DOSY-ECC-MW-determination parameters of MHMDS in TOL- $d_{8}(15 \mathrm{~mm})$ with ammonia as donor base. ADAM was used as internal reference $(15 \mathrm{mM})$ and $\mathrm{ECC}_{\mathrm{DSE}}$ to determine the MWs.

\begin{tabular}{lccc}
\hline & LiHMDS & NaHMDS & KHMDS \\
\hline$D_{\mathrm{x}}$ & $9.4180 \mathrm{E}-10$ & $8.8640 \mathrm{E}-10$ & $8.1480 \mathrm{E}-10$ \\
$\log D_{\mathrm{x}}$ & -9.0260 & -9.0524 & -9.0889 \\
$\log D_{\mathrm{x}, \text { norm }}$ & -9.0643 & -9.1356 & -9.1243 \\
$D_{\text {ref }}(\mathrm{ADAM})$ & $1.5590 \mathrm{E}-09$ & $1.7290 \mathrm{E}-09$ & $1.5485 \mathrm{E}-09$ \\
$\log D_{\text {ref }}(\mathrm{ADAM})$ & -8.8072 & -8.7622 & -8.8101 \\
$M W_{\text {det }}[\mathrm{g} / \mathrm{mol}]$ & 341 & 447 & 428
\end{tabular}


A-Table 14. DOSY-ECC-MW-determination of LiHMDS in TOL-d $(15 \mathrm{~mm})$ with ammonia as donor base. ADAM was used as internal reference $(15 \mathrm{mM})$ and $\mathrm{ECC}_{\mathrm{DSE}}$ to determine the MWs.

\begin{tabular}{ccccc}
\hline & Species & $\begin{array}{c}M W_{\text {calc }} \\
{[\mathrm{g} / \mathrm{mol}]}\end{array}$ & $\begin{array}{c}M W_{\text {err }} \\
{[\mathrm{g} / \mathrm{mol}]}\end{array}$ & $\begin{array}{c}M W_{\text {det }} \\
341 \mathrm{~g} / \mathrm{mol}\end{array}$ \\
\hline A & {$\left[\mathrm{LiHMDS}_{2}\right.$} & 335 & $-\mathbf{2}$ & \\
B & $\left(\mathrm{NH}_{3}\right) \cdot[\mathrm{LiHMDS}]_{2}$ & 352 & $\mathbf{3}$ & \\
C & $\left(\mathrm{NH}_{3}\right)_{2} \cdot[\mathrm{LiHMDS}]_{2}$ & 369 & 7 & \\
D & $\left(\mathrm{NH}_{3}\right)_{3} \cdot[\mathrm{LiHMDS}]_{2}$ & 386 & 12 & \\
E/F & $\left(\mathrm{NH}_{3}\right)_{4} \cdot[\mathrm{LiHMDS}]_{2}$ & 403 & 15 \\
G & $\left(\mathrm{NH}_{3}\right)_{5} \cdot[\mathrm{LiHMDS}]_{2}$ & 420 & 19 \\
H & $\left(\mathrm{NH}_{3}\right)_{3} \cdot$ LiHMDS & 218 & -56
\end{tabular}

A-Table 15. DOSY-ECC-MW-determination of NaHMDS in TOL-d $(15 \mathrm{~mm})$ with ammonia as donor base. ADAM was used as internal reference $(15 \mathrm{mM})$ and $\mathrm{ECC}_{\mathrm{DSE}}$ to determine the MWs.

\begin{tabular}{ccccc}
\hline & Species & $\begin{array}{c}M W_{\text {calc }} \\
{[\mathrm{g} / \mathrm{mol}]}\end{array}$ & $\begin{array}{c}M W_{\text {err }} \\
{[\mathrm{g} / \mathrm{mol}]}\end{array}$ & $\begin{array}{c}M W_{\text {det }} \\
447 \mathrm{~g} / \mathrm{mol}\end{array}$ \\
\hline A & {$[\mathrm{NaHMDS}]_{2}$} & 367 & -22 & \\
B & $\left(\mathrm{NH}_{3}\right) \cdot[\mathrm{NaHMDS}]_{2}$ & 384 & -16 & \\
C & $\left(\mathrm{NH}_{3}\right)_{2} \cdot[\mathrm{NaHMDS}]_{2}$ & 401 & -11 & -7 \\
D & $\left(\mathrm{NH}_{3}\right)_{3} \cdot[\mathrm{NaHMDS}]_{2}$ & 418 & $-\mathbf{2}$ & \\
E/F & $\left(\mathrm{NH}_{3}\right)_{4} \cdot[\mathrm{NaHMDS}]_{2}$ & 435 & $-\mathbf{3}$ & \\
G & $\left(\mathrm{NH}_{3}\right)_{5} \cdot[\mathrm{NaHMDS}]_{2}$ & 452 & $\mathbf{1}$ & \\
H & $\left(\mathrm{NH}_{3}\right)_{3} \cdot \mathrm{NaHMDS}$ & 234 & -90
\end{tabular}

A-Table 16. DOSY-ECC-MW-determination of KHMDS in TOL-d $(15 \mathrm{~mm})$ with ammonia as donor base. ADAM was used as internal reference $(15 \mathrm{mM})$ and $\mathrm{ECC}_{\mathrm{DSE}}$ to determine the MWs.

\begin{tabular}{ccccc}
\hline & Species & $\begin{array}{c}M W_{\text {calc }} \\
{[\mathrm{g} / \mathrm{mol}]}\end{array}$ & $\begin{array}{c}M W_{\text {err }} \\
{[\mathrm{g} / \mathrm{mol}]}\end{array}$ & $\begin{array}{c}M W_{\text {det }} \\
428 \mathrm{~g} / \mathrm{mol}\end{array}$ \\
\hline & & & & \\
A & {$\left[\mathrm{KHMDS}_{2}\right.$} & 399 & $-\mathbf{7}$ & \\
B & $\left(\mathrm{NH}_{3}\right) \cdot[\mathrm{KHMDS}]_{2}$ & 416 & $\mathbf{- 3}$ & \\
C & $\left(\mathrm{NH}_{3}\right)_{2} \cdot[\mathrm{KHMDS}]_{2}$ & 433 & $\mathbf{1}$ & \\
D & $\left(\mathrm{NH}_{3}\right)_{3} \cdot[\mathrm{KHMDS}]_{2}$ & 450 & $\mathbf{5}$ & \\
E/F & $\left(\mathrm{NH}_{3}\right)_{4} \cdot[\mathrm{KHMDS}]_{2}$ & 467 & $\mathbf{8}$ & \\
G & $\left(\mathrm{NH}_{3}\right)_{5} \cdot[\mathrm{KHMDS}]_{2}$ & 484 & 12 & \\
H & $\left(\mathrm{NH}_{3}\right)_{3} \cdot \mathrm{KHMDS}$ & 251 & -71
\end{tabular}




\section{${ }^{1} \mathrm{H}$ spectra of ${ }^{\mathrm{i}} \mathrm{Pr}_{2} \mathrm{NMgCl} 7$ in THF- $d_{8}$}

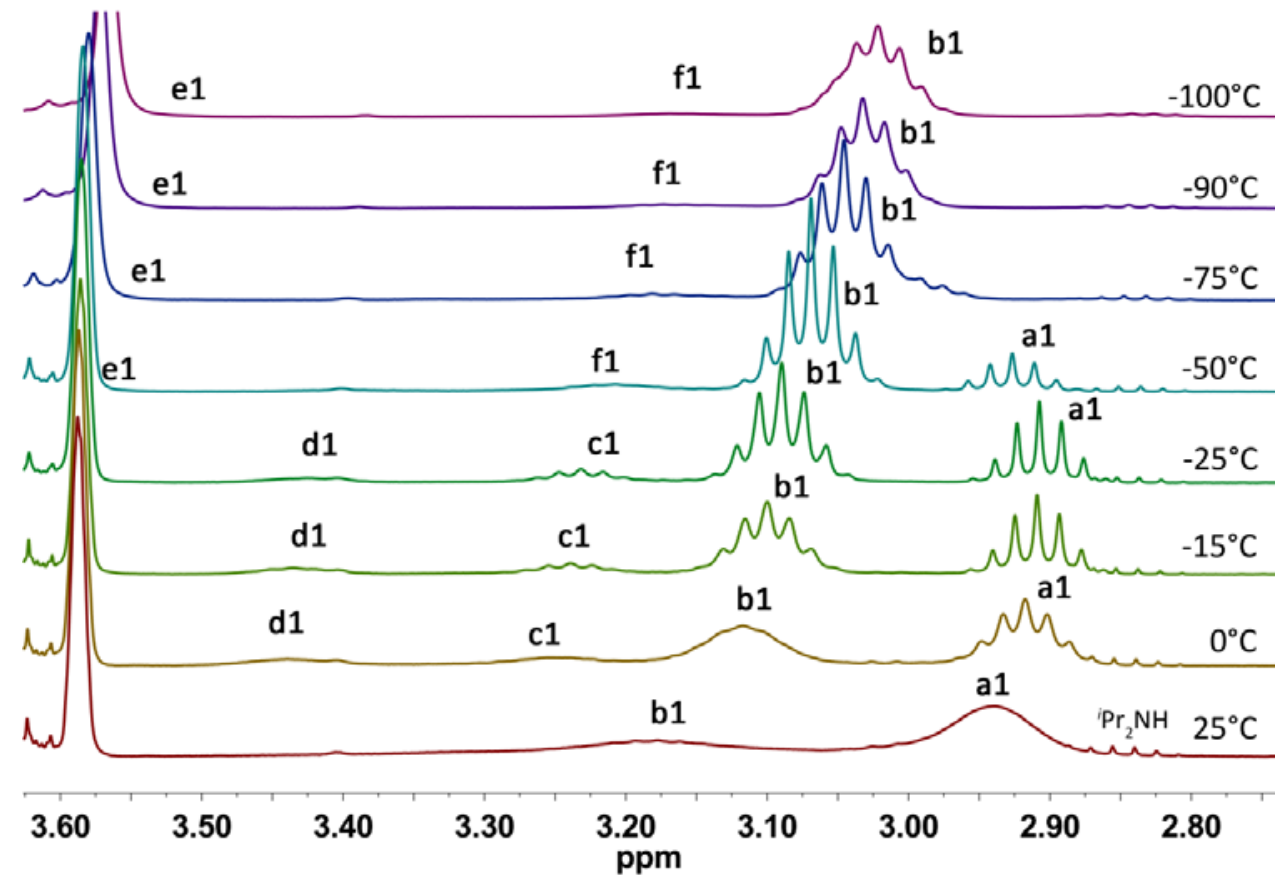

A-Figure 4. ${ }^{1} \mathrm{H}$ spectrum of ${ }^{\mathrm{i}} \mathrm{Pr}_{2} \mathrm{NMgCl} 7(0.10 \mathrm{M})$ in THF- $d_{8}$. ( $\alpha-\mathrm{CH}$ region).

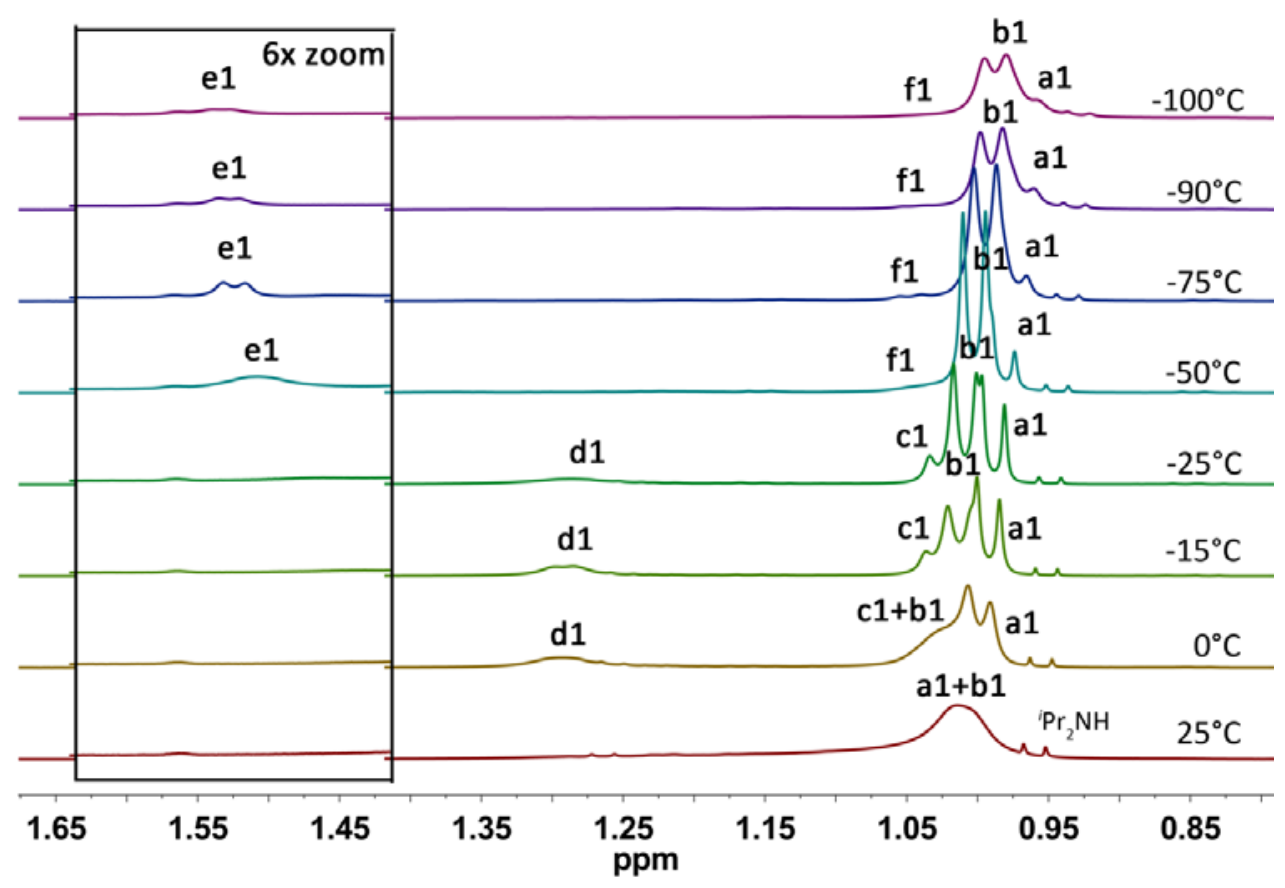

A-Figure 5. ${ }^{1} \mathrm{H}$ spectrum of ${ }^{\mathrm{i}} \mathrm{Pr}_{2} \mathrm{NMgCl} 7(0.10 \mathrm{M})$ in THF- $d_{8} .\left(-\mathrm{CH}_{3}\right.$ region $)$. 


\section{${ }^{1} \mathrm{H}$ and ${ }^{7} \mathrm{Li}$ spectra of ${ }^{\mathrm{i}} \mathrm{Pr}_{2} \mathrm{NMgCl} \cdot \mathrm{LiCl} 9$ in THF- $d_{8}$}

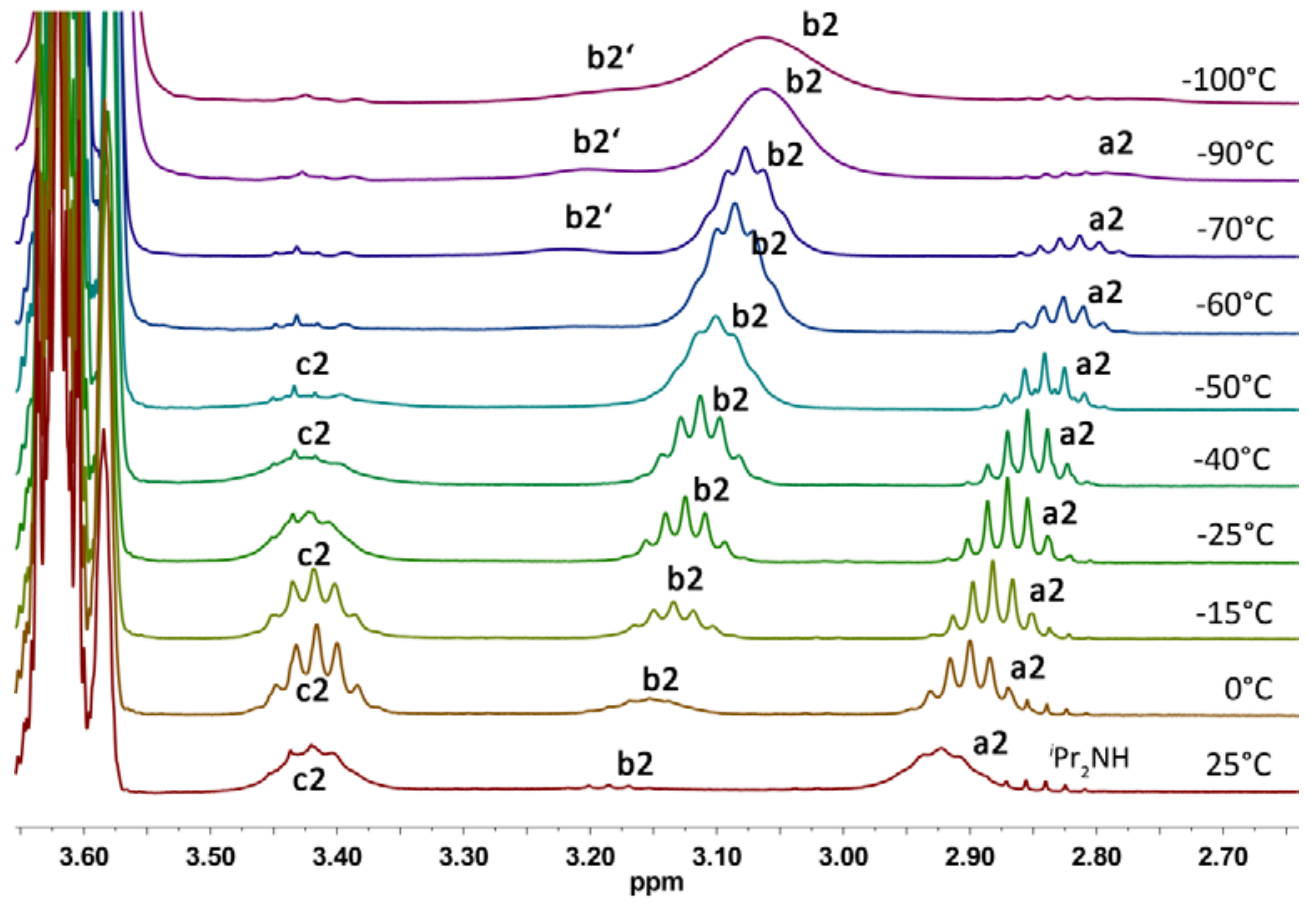

A-Figure 6. ${ }^{1} \mathrm{H}$ spectrum of ${ }^{i} \operatorname{Pr}_{2} \mathrm{NMgCl} \cdot \mathrm{LiCl} 9(0.10 \mathrm{M})$ in $\mathrm{THF}-d_{8} .(\alpha-\mathrm{CH}$ region).

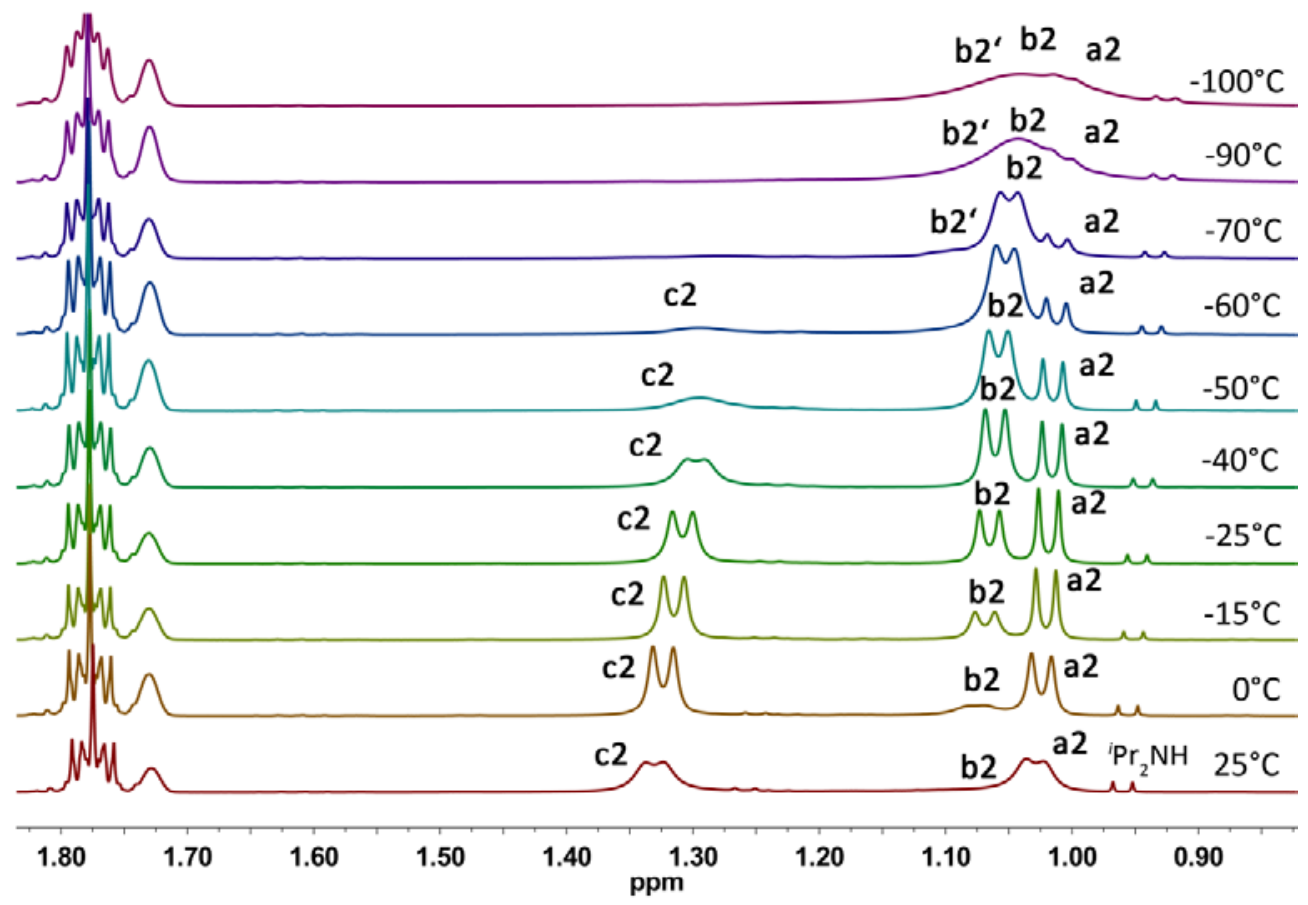

A-Figure 7. ${ }^{1} \mathrm{H}$ spectrum of ${ }^{i} \mathrm{Pr}_{2} \mathrm{NMgCl} \cdot \mathrm{LiCl} 9(0.10 \mathrm{M})$ in $\mathrm{THF}-d_{8} .\left(-\mathrm{CH}_{3}\right.$ region). 


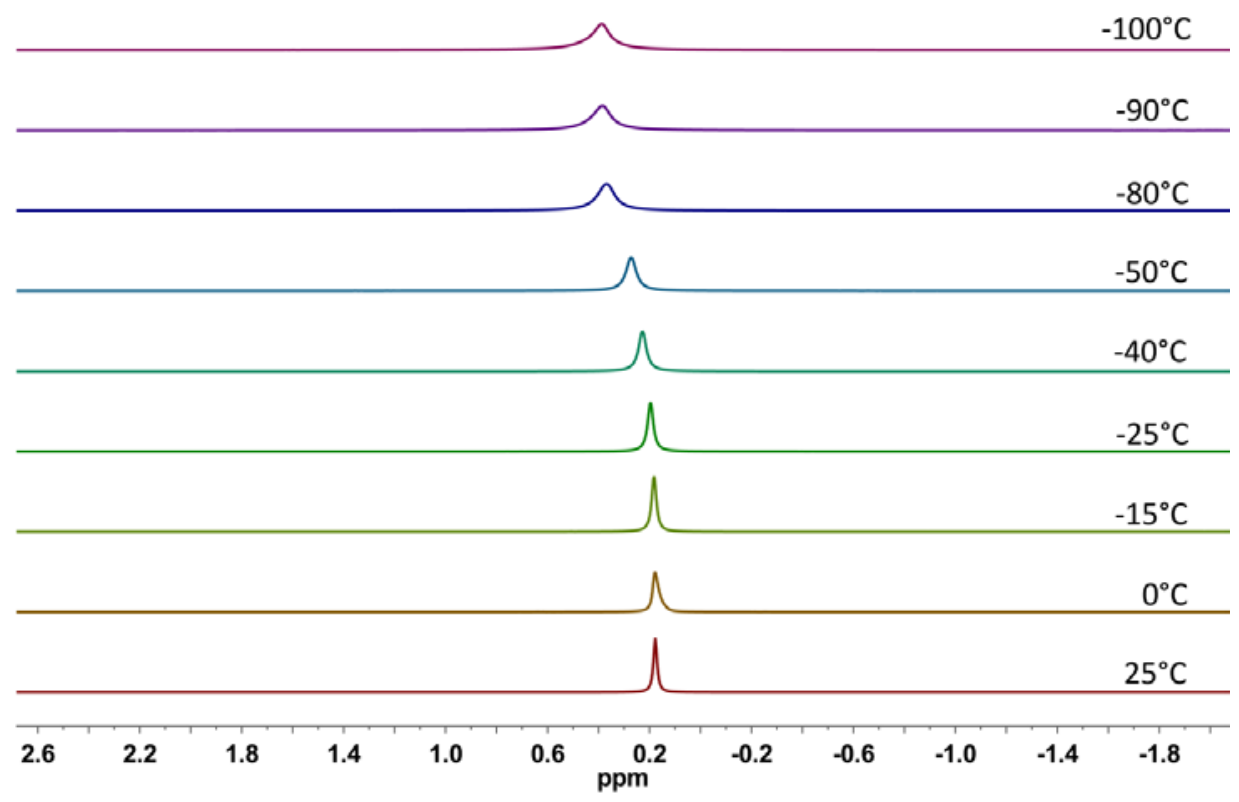

A-Figure 8. ${ }^{7} \mathrm{Li}$ spectra of ${ }^{i} \operatorname{Pr}_{2} \mathrm{NMgCl} \cdot \mathrm{LiCl} 9(0.10 \mathrm{M})$ in THF- $d_{8}$.

\section{${ }^{7} \mathrm{Li}$ Spectra of $\mathrm{LiCl}$ in THF- $d_{8}$}

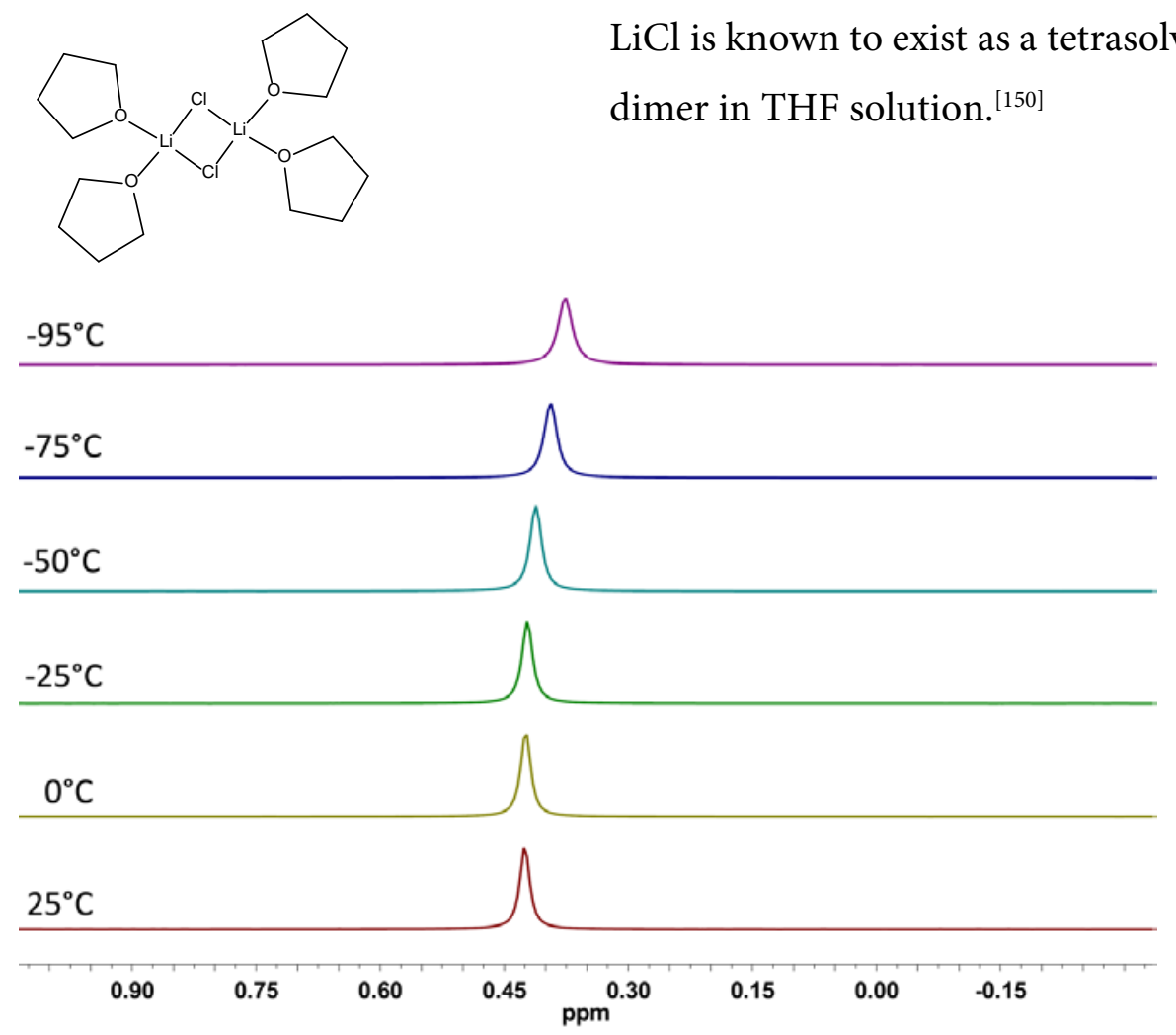

A-Figure 9. ${ }^{7} \mathrm{Li}$ spectra of $\mathrm{LiCl}(0.03 \mathrm{M})$ in THF- $d_{8}$. 


\section{${ }^{1} \mathrm{H}$ and ${ }^{7} \mathrm{Li}$ Spectra of Lithiumdiisopropylamide (LDA) in THF- $d_{8}$}

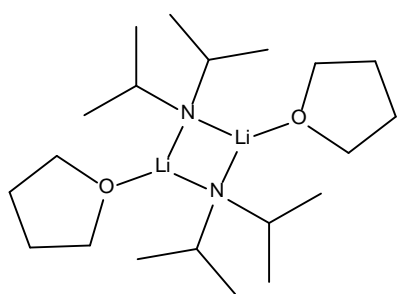

LDA is known to exist exclusively as a disolvated dimer in THF solution. ${ }^{[151]}$

Lithium species of 9 resonances at a chemical shift region of $0.18 \mathrm{ppm}$ to $0.38 \mathrm{ppm}$ in the ${ }^{7} \mathrm{Li}$ experiment.

LDA can be excluded to be present in solution of $\mathbf{9}$, because it resonances at around $2 \mathrm{ppm}$.

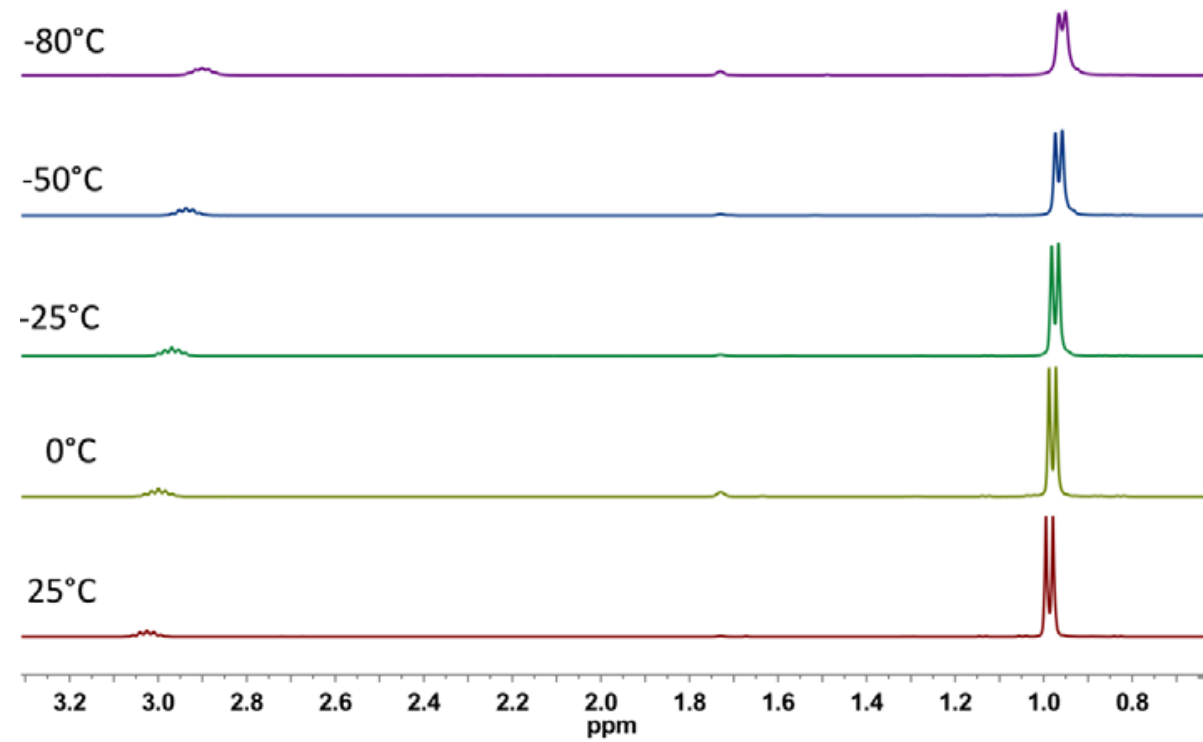

A-Figure 10. ${ }^{1} \mathrm{H}$ spectrum of LDA $(0.10 \mathrm{M})$ in THF- $d_{8}$. 


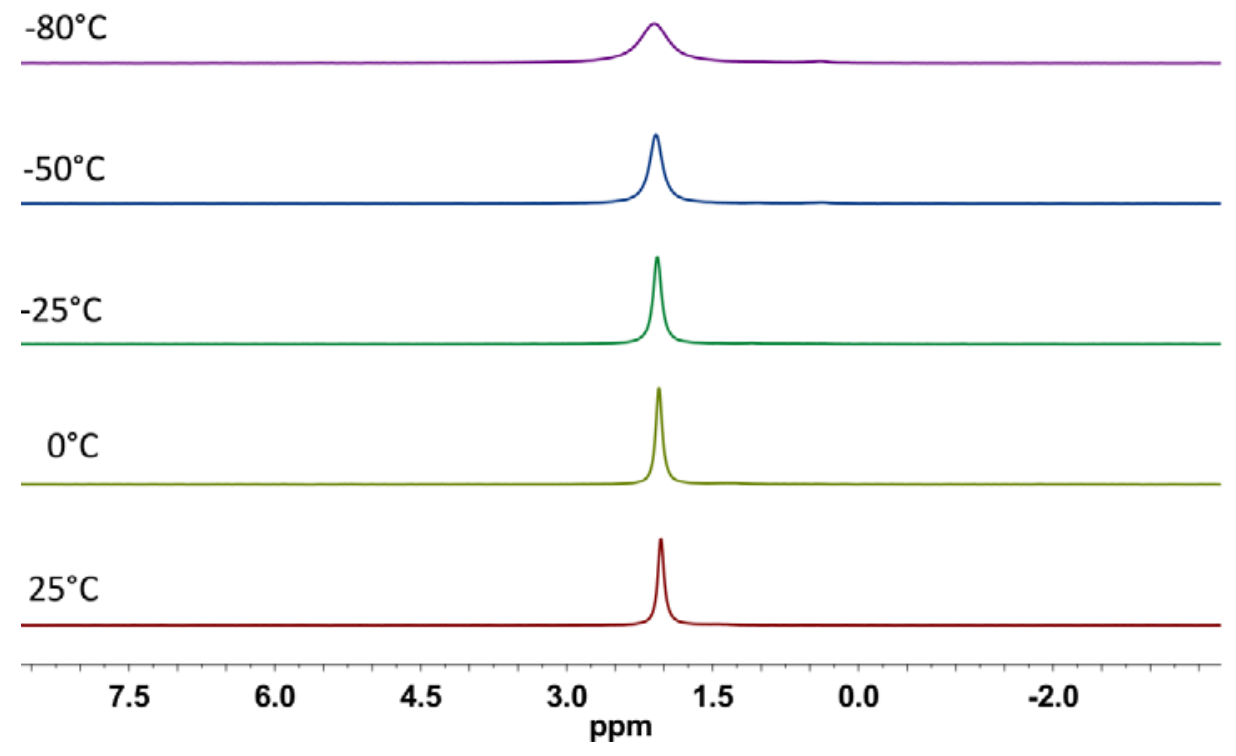

A-Figure 11. ${ }^{7} \mathrm{Li}$ spectrum of LDA $(0.10 \mathrm{M})$ in $\mathrm{THF}-d_{8}$.

\section{${ }^{1} \mathrm{H}$-DOSY-ECC-MW-determination parameters of ${ }^{\mathrm{i}} \mathrm{Pr}_{2} \mathrm{NMgCl} 7$}

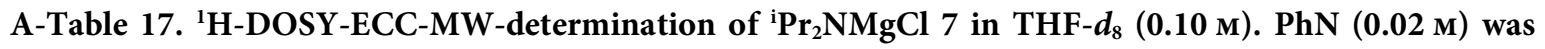
used as internal reference with $\log D_{\text {ref,fix }}(\mathrm{PhN})=\mathbf{- 8 . 8 8 1 2}$. The accuracy of this method is in the range of $M W_{\text {err }}< \pm 9 \%$.

\begin{tabular}{|c|c|c|c|c|c|}
\hline \multirow[t]{2}{*}{${ }^{1} \mathrm{H}-\mathrm{DOSY}$} & \multicolumn{5}{|r|}{$25^{\circ} \mathrm{C}$} \\
\hline & Signal & a & Species & $M W_{\text {calc }}[\mathrm{g} / \mathrm{mol}]$ & $M W_{\mathrm{err}}[\%]$ \\
\hline$D_{\mathrm{x}}(\mathrm{a})$ & $1.1250 \mathrm{E}-09$ & & $\mathrm{MgCl}_{2} \cdot \mathrm{D} 1(\mathrm{D} 2) \cdot \mathrm{MgCl}_{2}$ & 655 & 50 \\
\hline $\log D_{\mathrm{x}}(\mathrm{a})$ & -8.9488 & & D1 & 464 & 30 \\
\hline $\log D_{\mathrm{x}, \text { norm }}(\mathrm{a})$ & -8.9999 & & $\mathrm{M} 1 \cdot\left(\mathrm{MgCl}_{2}\right)_{2} \cdot \mathrm{M} 1$ & 943 & 65 \\
\hline$D_{\text {ref }}(\mathrm{PhN})$ & $1.4787 \mathrm{E}-09$ & & $(\mu-\mathrm{Cl} 3)-\mathrm{M} 1 \cdot \mathrm{MgCl} 2$ & 616 & 47 \\
\hline $\log D_{\text {ref }}(\mathrm{PhN})$ & -8.8301 & & $\mathrm{D} 2$ & 464 & 30 \\
\hline \multirow[t]{3}{*}{$M W_{\text {det }}[\mathrm{g} / \mathrm{mol}]$} & 326 & & M2 & 369 & 12 \\
\hline & & & M1 & 304 & -7 \\
\hline & Signal & $\mathrm{b}$ & Species & $M W_{\text {calc }}[\mathrm{g} / \mathrm{mol}]$ & $M W_{\text {err }}[\%]$ \\
\hline$D_{\mathrm{x}}(\mathrm{b})$ & $1.0600 \mathrm{E}-09$ & & $\mathrm{MgCl}_{2} \cdot \mathrm{D} 1(\mathrm{D} 2) \cdot \mathrm{MgCl}_{2}$ & 655 & 45 \\
\hline $\log D_{\mathrm{x}}(\mathrm{b})$ & -8.9747 & & D1 & 464 & 22 \\
\hline $\log D_{x, \text { norm }}(\mathrm{b})$ & -9.0258 & & $\mathrm{M} 1 \cdot\left(\mathrm{MgCl}_{2}\right)_{2} \cdot \mathrm{M} 1$ & 943 & 62 \\
\hline$D_{\text {ref }}(\mathrm{PhN})$ & $1.4787 \mathrm{E}-09$ & & $(\mu-\mathrm{Cl} 3)-\mathrm{M} 1 \cdot \mathrm{MgCl} 2$ & 616 & 41 \\
\hline $\log D_{\text {ref }}(\mathrm{PhN})$ & -8.8301 & & D2 & 464 & 22 \\
\hline \multirow[t]{2}{*}{$M W_{\text {det }}[\mathrm{g} / \mathrm{mol}]$} & 361 & & M2 & 369 & 2 \\
\hline & & & M1 & 304 & -19 \\
\hline
\end{tabular}




\begin{tabular}{|c|c|c|c|c|c|}
\hline & Signal & $\mathrm{a}$ & Species & $M W_{\text {calc }}[\mathrm{g} / \mathrm{mol}]$ & $\begin{array}{c}-15^{\circ} \mathrm{C} \\
M W_{\text {err }}[\%]\end{array}$ \\
\hline$D_{\mathrm{x}}(\mathrm{a})$ & $5.3190 \mathrm{E}-10$ & & $\mathrm{MgCl}_{2} \cdot \mathrm{D} 1(\mathrm{D} 2) \cdot \mathrm{MgCl}_{2}$ & 655 & 53 \\
\hline $\log D_{\mathrm{x}}(\mathrm{a})$ & -9.2742 & & $\mathrm{D} 1$ & 464 & 34 \\
\hline $\log D_{\mathrm{x}, \text { norm }}(\mathrm{a})$ & -8.9833 & & $\mathrm{M} 1 \cdot\left(\mathrm{MgCl}_{2}\right)_{2} \cdot \mathrm{M} 1$ & 943 & 68 \\
\hline$D_{\text {ref }}(\mathrm{PhN})$ & $6.7280 \mathrm{E}-10$ & & $(\mu-\mathrm{Cl} 3)-\mathrm{M} 1 \cdot \mathrm{MgCl} 2$ & 616 & 50 \\
\hline $\log D_{\text {ref }}(\mathrm{PhN})$ & -9.1721 & & D2 & 464 & 34 \\
\hline \multirow[t]{3}{*}{$M W_{\text {det }}[\mathrm{g} / \mathrm{mol}]$} & 305 & & M2 & 369 & 17 \\
\hline & & & M1 & 304 & 0 \\
\hline & Signal & $\mathrm{b}$ & Species & $M W_{\text {calc }}[\mathrm{g} / \mathrm{mol}]$ & $M W_{\mathrm{err}}[\%]$ \\
\hline$D_{\mathrm{x}}(\mathrm{b})$ & $4.6230 \mathrm{E}-10$ & & $\mathrm{MgCl}_{2} \cdot \mathrm{D} 1(\mathrm{D} 2) \cdot \mathrm{MgCl}_{2}$ & 655 & 41 \\
\hline $\log D_{\mathrm{x}}(\mathrm{b})$ & -9.3351 & & D1 & 464 & 16 \\
\hline $\log D_{\mathrm{x}, \text { norm }}(\mathrm{b})$ & -9.0442 & & $\mathrm{M} 1 \cdot\left(\mathrm{MgCl}_{2}\right)_{2} \cdot \mathrm{M} 1$ & 943 & 59 \\
\hline$D_{\text {ref }}(\mathrm{PhN})$ & $6.7280 \mathrm{E}-10$ & & $(\mu-\mathrm{Cl} 3)-\mathrm{M} 1 \cdot \mathrm{MgCl} 2$ & 616 & 37 \\
\hline $\log D_{\text {ref }}(\mathrm{PhN})$ & -9.1721 & & $\mathrm{D} 2$ & 464 & 16 \\
\hline \multirow[t]{3}{*}{$M W_{\text {det }}[\mathrm{g} / \mathrm{mol}]$} & 389 & & M2 & 369 & -5 \\
\hline & & & M1 & 304 & -28 \\
\hline & Signal & c & Species & $M W_{\text {calc }}[\mathrm{g} / \mathrm{mol}]$ & $M W_{\mathrm{err}}[\%]$ \\
\hline$D_{\mathrm{x}}(\mathrm{c})$ & $4.2840 \mathrm{E}-10$ & & $\mathrm{MgCl}_{2} \cdot \mathrm{D} 1(\mathrm{D} 2) \cdot \mathrm{MgCl}_{2}$ & 655 & 32 \\
\hline $\log D_{\mathrm{x}}(\mathrm{c})$ & -9.3682 & & D1 & 464 & 5 \\
\hline $\log D_{\mathrm{x}, \text { norm }}(\mathrm{c})$ & -9.0772 & & $\mathrm{M} 1 \cdot\left(\mathrm{MgCl}_{2}\right)_{2} \cdot \mathrm{M} 1$ & 943 & 53 \\
\hline$D_{\text {ref }}(\mathrm{PhN})$ & $6.7280 \mathrm{E}-10$ & & $(\mu-\mathrm{Cl} 3)-\mathrm{M} 1 \cdot \mathrm{MgCl} 2$ & 616 & 28 \\
\hline $\log D_{\text {ref }}(\mathrm{PhN})$ & -9.1721 & & $\mathrm{D} 2$ & 464 & 5 \\
\hline \multirow[t]{3}{*}{$M W_{\text {det }}[\mathrm{g} / \mathrm{mol}]$} & 443 & & M2 & 369 & -20 \\
\hline & & & M1 & 304 & -46 \\
\hline & Signal & $\mathrm{d}$ & Species & $M W_{\text {calc }}[\mathrm{g} / \mathrm{mol}]$ & $M W_{\text {err }}[\%]$ \\
\hline$D_{\mathrm{x}}(\mathrm{d})$ & $4.3580 \mathrm{E}-10$ & & $\mathrm{MgCl}_{2} \cdot \mathrm{D} 1(\mathrm{D} 2) \cdot \mathrm{MgCl}_{2}$ & 655 & 34 \\
\hline $\log D_{\mathrm{x}}(\mathrm{d})$ & -9.3607 & & D1 & 464 & 7 \\
\hline $\log D_{\mathrm{x}, \text { norm }}(\mathrm{d})$ & -9.0698 & & $\mathrm{M} 1 \cdot\left(\mathrm{MgCl}_{2}\right)_{2} \cdot \mathrm{M} 1$ & 943 & 54 \\
\hline$D_{\text {ref }}(\mathrm{PhN})$ & $6.7280 \mathrm{E}-10$ & & $(\mu-\mathrm{Cl} 3)-\mathrm{M} 1 \cdot \mathrm{MgCl} 2$ & 616 & 30 \\
\hline $\log D_{\text {ref }}(\mathrm{PhN})$ & -9.1721 & & $\mathrm{D} 2$ & 464 & 7 \\
\hline \multirow[t]{4}{*}{$M W_{\text {det }}[\mathrm{g} / \mathrm{mol}]$} & 430 & & M2 & 369 & -17 \\
\hline & & & M1 & 304 & -41 \\
\hline & & & & & $-25^{\circ} \mathrm{C}$ \\
\hline & Signal & $\mathrm{a}$ & Species & $M W_{\text {calc }}[\mathrm{g} / \mathrm{mol}]$ & $M W_{\text {err }}[\%]$ \\
\hline$D_{\mathrm{x}}(\mathrm{a})$ & $4.2320 \mathrm{E}-10$ & & $\mathrm{MgCl}_{2} \cdot \mathrm{D} 1(\mathrm{D} 2) \cdot \mathrm{MgCl}_{2}$ & 655 & 52 \\
\hline $\log D_{\mathrm{x}}(\mathrm{a})$ & -9.3735 & & D1 & 464 & 33 \\
\hline $\log D_{\mathrm{x}, \text { norm }}(\mathrm{a})$ & -8.9883 & & $\mathrm{M} 1 \cdot\left(\mathrm{MgCl}_{2}\right)_{2} \cdot \mathrm{M} 1$ & 943 & 67 \\
\hline$D_{\text {ref }}(\mathrm{PhN})$ & $5.4157 \mathrm{E}-10$ & & $(\mu-\mathrm{Cl} 3)-\mathrm{M} 1 \cdot \mathrm{MgCl} 2$ & 616 & 49 \\
\hline
\end{tabular}




\begin{tabular}{|c|c|c|c|c|c|}
\hline $\log D_{\text {ref }}(\mathrm{PhN})$ & -9.2663 & & D2 & 464 & 33 \\
\hline \multirow[t]{3}{*}{$M W_{\text {det }}[\mathrm{g} / \mathrm{mol}]$} & 312 & & M2 & 369 & 16 \\
\hline & & & M1 & 304 & -2 \\
\hline & Signal & $\mathrm{b}$ & Species & $M W_{\text {calc }}[\mathrm{g} / \mathrm{mol}]$ & $M W_{\text {err }}[\%]$ \\
\hline$D_{\mathrm{x}}(\mathrm{b})$ & $3.8110 \mathrm{E}-10$ & & $\mathrm{MgCl}_{2} \cdot \mathrm{D} 1(\mathrm{D} 2) \cdot \mathrm{MgCl}_{2}$ & 655 & 43 \\
\hline $\log D_{\mathrm{x}}(\mathrm{b})$ & -9.4190 & & $\mathrm{D} 1$ & 464 & 20 \\
\hline $\log D_{\mathrm{x}, \text { norm }}(\mathrm{b})$ & -9.0338 & & $\mathrm{M} 1 \cdot\left(\mathrm{MgCl}_{2}\right)_{2} \cdot \mathrm{M} 1$ & 943 & 60 \\
\hline$D_{\text {ref }}(\mathrm{PhN})$ & $5.4157 \mathrm{E}-10$ & & $(\mu-\mathrm{Cl} 3)-\mathrm{M} 1 \cdot \mathrm{MgCl} 2$ & 616 & 39 \\
\hline $\log D_{\text {ref }}(\mathrm{PhN})$ & -9.2663 & & $\mathrm{D} 2$ & 464 & 20 \\
\hline \multirow[t]{3}{*}{$M W_{\operatorname{det}}[\mathrm{g} / \mathrm{mol}]$} & 373 & & M2 & 369 & -1 \\
\hline & & & M1 & 304 & -23 \\
\hline & Signal & c & Species & $M W_{\text {calc }}[\mathrm{g} / \mathrm{mol}]$ & $M W_{\text {err }}[\%]$ \\
\hline$D_{\mathrm{x}}(\mathrm{c})$ & $3.3890 \mathrm{E}-10$ & & $\mathrm{MgCl}_{2} \cdot \mathrm{D} 1(\mathrm{D} 2) \cdot \mathrm{MgCl}_{2}$ & 655 & 30 \\
\hline $\log D_{\mathrm{x}}(\mathrm{c})$ & -9.4699 & & D1 & 464 & 2 \\
\hline $\log D_{\mathrm{x}, \text { norm }}(\mathrm{c})$ & -9.0848 & & $\mathrm{M} 1 \cdot\left(\mathrm{MgCl}_{2}\right)_{2} \cdot \mathrm{M} 1$ & 943 & 52 \\
\hline$D_{\text {ref }}(\mathrm{PhN})$ & $5.4157 \mathrm{E}-10$ & & $(\mu-\mathrm{Cl} 3)-\mathrm{M} 1 \cdot \mathrm{MgCl} 2$ & 616 & 26 \\
\hline $\log D_{\text {ref }}(\mathrm{PhN})$ & -9.2663 & & $\mathrm{D} 2$ & 464 & 2 \\
\hline \multirow[t]{3}{*}{$M W_{\text {det }}[\mathrm{g} / \mathrm{mol}]$} & 456 & & M2 & 369 & -24 \\
\hline & & & M1 & 304 & -50 \\
\hline & Signal & $\mathrm{d}$ & Species & $M W_{\text {calc }}[\mathrm{g} / \mathrm{mol}]$ & $M W_{\text {err }}[\%]$ \\
\hline$D_{\mathrm{x}}(\mathrm{d})$ & $3.4580 \mathrm{E}-10$ & & $\mathrm{MgCl}_{2} \cdot \mathrm{D} 1(\mathrm{D} 2) \cdot \mathrm{MgCl}_{2}$ & 655 & 33 \\
\hline $\log D_{\mathrm{x}}(\mathrm{d})$ & -9.4612 & & D1 & 464 & 5 \\
\hline $\log D_{\mathrm{x}, \text { norm }}(\mathrm{d})$ & -9.0760 & & $\mathrm{M} 1 \cdot\left(\mathrm{MgCl}_{2}\right)_{2} \cdot \mathrm{M} 1$ & 943 & 53 \\
\hline$D_{\text {ref }}(\mathrm{PhN})$ & $5.4157 \mathrm{E}-10$ & & $(\mu-\mathrm{Cl} 3)-\mathrm{M} 1 \cdot \mathrm{MgCl} 2$ & 616 & 28 \\
\hline $\log D_{\text {ref }}(\mathrm{PhN})$ & -9.2663 & & $\mathrm{D} 2$ & 464 & 5 \\
\hline \multirow[t]{4}{*}{$M W_{\text {det }}[\mathrm{g} / \mathrm{mol}]$} & 441 & & M2 & 369 & -20 \\
\hline & & & M1 & 304 & -45 \\
\hline & & & & & $-50^{\circ} \mathrm{C}$ \\
\hline & Signal & $\mathrm{a}$ & Species & $M W_{\text {calc }}[\mathrm{g} / \mathrm{mol}]$ & $M W_{\text {err }}[\%]$ \\
\hline$D_{\mathrm{x}}(\mathrm{a})$ & $2.3360 \mathrm{E}-10$ & & $\mathrm{MgCl}_{2} \cdot \mathrm{D} 1(\mathrm{D} 2) \cdot \mathrm{MgCl}_{2}$ & 655 & 53 \\
\hline $\log D_{\mathrm{x}}(\mathrm{a})$ & -9.6315 & & D1 & 464 & 33 \\
\hline $\log D_{\mathrm{x}, \text { norm }}(\mathrm{a})$ & -8.9862 & & $\mathrm{M} 1 \cdot\left(\mathrm{MgCl}_{2}\right)_{2} \cdot \mathrm{M} 1$ & 943 & 67 \\
\hline$D_{\text {ref }}(\mathrm{PhN})$ & $2.9747 \mathrm{E}-10$ & & $(\mu-\mathrm{Cl} 3)-\mathrm{M} 1 \cdot \mathrm{MgCl} 2$ & 616 & 50 \\
\hline $\log D_{\text {ref }}(\mathrm{PhN})$ & -9.5266 & & D2 & 464 & 33 \\
\hline \multirow[t]{2}{*}{$M W_{\text {det }}[\mathrm{g} / \mathrm{mol}]$} & 309 & & M2 & 369 & 16 \\
\hline & & & M1 & 304 & -2 \\
\hline
\end{tabular}




\begin{tabular}{|c|c|c|c|c|c|}
\hline & Signal & $\mathrm{b}$ & Species & $M W_{\text {calc }}[\mathrm{g} / \mathrm{mol}]$ & $M W_{\text {err }}[\%]$ \\
\hline$D_{\mathrm{x}}(\mathrm{b})$ & $2.1800 \mathrm{E}-10$ & & $\mathrm{MgCl}_{2} \cdot \mathrm{D} 1(\mathrm{D} 2) \cdot \mathrm{MgCl}_{2}$ & 655 & 47 \\
\hline $\log D_{\mathrm{x}}(\mathrm{b})$ & -9.6615 & & D1 & 464 & 25 \\
\hline $\log D_{\mathrm{x}, \text { norm }}(\mathrm{b})$ & -9.0162 & & $\mathrm{M} 1 \cdot\left(\mathrm{MgCl}_{2}\right)_{2} \cdot \mathrm{M} 1$ & 943 & 63 \\
\hline$D_{\text {ref }}(\mathrm{PhN})$ & $2.9747 \mathrm{E}-10$ & & $(\mu-\mathrm{Cl} 3)-\mathrm{M} 1 \cdot \mathrm{MgCl} 2$ & 616 & 43 \\
\hline $\log D_{\text {ref }}(\mathrm{PhN})$ & -9.5266 & & D2 & 464 & 25 \\
\hline \multirow[t]{3}{*}{$M W_{\text {det }}[\mathrm{g} / \mathrm{mol}]$} & 348 & & M2 & 369 & 6 \\
\hline & & & M1 & 304 & -14 \\
\hline & Signal & $\mathrm{c}+\mathrm{d}$ & no signal & & \\
\hline
\end{tabular}

\begin{tabular}{|c|c|c|c|c|c|}
\hline & Signal & $\mathrm{e}$ & Species & $M W_{\text {calc }}[\mathrm{g} / \mathrm{mol}]$ & $M W_{\text {err }}[\%]$ \\
\hline$D_{\mathrm{x}}(\mathrm{e})$ & $1.9850 \mathrm{E}-10$ & & $\mathrm{MgCl}_{2} \cdot \mathrm{D} 1(\mathrm{D} 2) \cdot \mathrm{MgCl}_{2}$ & 655 & 24 \\
\hline $\log D_{\mathrm{x}}(\mathrm{e})$ & -9.7022 & & D1 & 464 & -7 \\
\hline $\log D_{\mathrm{x}, \text { norm }}(\mathrm{e})$ & -9.0569 & & $\mathrm{M} 1 \cdot\left(\mathrm{MgCl}_{2}\right)_{2} \cdot \mathrm{M} 1$ & 943 & 47 \\
\hline$D_{\text {ref }}(\mathrm{PhN})$ & $2.9747 \mathrm{E}-10$ & & $(\mu-\mathrm{Cl} 3)-\mathrm{M} 1 \cdot \mathrm{MgCl}_{2}$ & 616 & 19 \\
\hline $\log D_{\text {ref }}(\mathrm{PhN})$ & -9.5266 & & $\mathrm{D} 2$ & 464 & -7 \\
\hline$M W_{\text {det,corr }}[\mathrm{g} / \mathrm{mol}]$ & 497 & & M2 & 369 & -35 \\
\hline \multirow[t]{2}{*}{$M W_{\text {det }}[\mathrm{g} / \mathrm{mol}]$} & 409 & & M1 & 304 & -63 \\
\hline & Signal & $\mathrm{f}$ & Species & $M W_{\text {calc }}[\mathrm{g} / \mathrm{mol}]$ & $M W_{\text {err }}[\%]$ \\
\hline$D_{\mathrm{x}}(\mathrm{f})$ & $1.9330 \mathrm{E}-10$ & & $\mathrm{MgCl}_{2} \cdot \mathrm{D} 1(\mathrm{D} 2) \cdot \mathrm{MgCl}_{2}$ & 655 & 35 \\
\hline $\log D_{\mathrm{x}}(\mathrm{f})$ & -9.7138 & & D1 & 464 & 8 \\
\hline $\log D_{x, \text { norm }}(f)$ & -9.0684 & & $\mathrm{M} 1 \cdot\left(\mathrm{MgCl}_{2}\right)_{2} \cdot \mathrm{M} 1$ & 943 & 55 \\
\hline$D_{\text {ref }}(\mathrm{PhN})$ & $2.9747 \mathrm{E}-10$ & & $(\mu-\mathrm{Cl} 3)-\mathrm{M} 1 \cdot \mathrm{MgCl} 2$ & 616 & 31 \\
\hline $\log D_{\text {ref }}(\mathrm{PhN})$ & -9.5266 & & $\mathrm{D} 2$ & 464 & 8 \\
\hline \multirow[t]{4}{*}{$M W_{\text {det }}[\mathrm{g} / \mathrm{mol}]$} & 428 & & M2 & 369 & -16 \\
\hline & & & M1 & 304 & -41 \\
\hline & & & & & $-70^{\circ} \mathrm{C}$ \\
\hline & Signal & $\mathrm{a}$ & Species & $M W_{\text {calc }}[\mathrm{g} / \mathrm{mol}]$ & $M W_{\mathrm{err}}[\%]$ \\
\hline$D_{\mathrm{x}}(\mathrm{a})$ & $1.1650 \mathrm{E}-10$ & & $\mathrm{MgCl}_{2} \cdot \mathrm{D} 1(\mathrm{D} 2) \cdot \mathrm{MgCl}_{2}$ & 655 & 55 \\
\hline $\log D_{\mathrm{x}}(\mathrm{a})$ & -9.9337 & & D1 & 464 & 36 \\
\hline $\log D_{\mathrm{x}, \text { norm }}(\mathrm{a})$ & -8.9763 & & $\mathrm{M} 1 \cdot\left(\mathrm{MgCl}_{2}\right)_{2} \cdot \mathrm{M} 1$ & 943 & 68 \\
\hline$D_{\text {ref }}(\mathrm{PhN})$ & $1.4503 \mathrm{E}-10$ & & $(\mu-\mathrm{Cl} 3)-\mathrm{M} 1 \cdot \mathrm{MgCl} 2$ & 616 & 52 \\
\hline $\log D_{\text {ref }}(\mathrm{PhN})$ & -9.8385 & & $\mathrm{D} 2$ & 464 & 36 \\
\hline \multirow[t]{2}{*}{$M W_{\text {det }}[\mathrm{g} / \mathrm{mol}]$} & 297 & & M2 & 369 & 19 \\
\hline & & & M1 & 304 & 2 \\
\hline
\end{tabular}




\begin{tabular}{|c|c|c|c|c|c|}
\hline & Signal & $\mathrm{b}$ & Species & $M W_{\text {calc }}[\mathrm{g} / \mathrm{mol}]$ & $M W_{\mathrm{err}}[\%]$ \\
\hline$D_{\mathrm{x}}(\mathrm{b})$ & $1.0720 \mathrm{E}-10$ & & $\mathrm{MgCl}_{2} \cdot \mathrm{D} 1(\mathrm{D} 2) \cdot \mathrm{MgCl}_{2}$ & 655 & 48 \\
\hline $\log D_{\mathrm{x}}(\mathrm{b})$ & -9.9698 & & D1 & 464 & 26 \\
\hline $\log D_{\mathrm{x}, \text { norm }}(\mathrm{b})$ & -9.0125 & & $\mathrm{M} 1 \cdot\left(\mathrm{MgCl}_{2}\right)_{2} \cdot \mathrm{M} 1$ & 943 & 64 \\
\hline$D_{\text {ref }}(\mathrm{PhN})$ & $1.4503 \mathrm{E}-10$ & & $(\mu-\mathrm{Cl} 3)-\mathrm{M} 1 \cdot \mathrm{MgCl} 2$ & 616 & 44 \\
\hline $\log D_{\text {ref }}(\mathrm{PhN})$ & -9.8385 & & $\mathrm{D} 2$ & 464 & 26 \\
\hline \multirow[t]{3}{*}{$M W_{\text {det }}[\mathrm{g} / \mathrm{mol}]$} & 343 & & M2 & 369 & 7 \\
\hline & & & M1 & 304 & -13 \\
\hline & Signal & $c+d$ & no signal & & \\
\hline
\end{tabular}

\begin{tabular}{|c|c|c|c|c|c|}
\hline & Signal & e & Species & $M W_{\text {calc }}[\mathrm{g} / \mathrm{mol}]$ & $M W_{\text {err }}[\%]$ \\
\hline$D_{\mathrm{x}}(\mathrm{e})$ & $9.1980 \mathrm{E}-11$ & & $\mathrm{MgCl}_{2} \cdot \mathrm{D} 1(\mathrm{D} 2) \cdot \mathrm{MgCl}_{2}$ & 655 & 17 \\
\hline $\log D_{\mathrm{x}}(\mathrm{e})$ & $-1.0036 \mathrm{E}+01$ & & D1 & 464 & -17 \\
\hline $\log D_{\mathrm{x}, \text { norm }}(\mathrm{e})$ & -9.0790 & & $\mathrm{M} 1 \cdot\left(\mathrm{MgCl}_{2}\right)_{2} \cdot \mathrm{M} 1$ & 943 & 42 \\
\hline$D_{\text {ref }}(\mathrm{PhN})$ & $1.4503 \mathrm{E}-10$ & & $(\mu-\mathrm{Cl} 3)-\mathrm{M} 1 \cdot \mathrm{MgCl}_{2}$ & 616 & 12 \\
\hline $\log D_{\text {ref }}(\mathrm{PhN})$ & -9.8385 & & $\mathrm{D} 2$ & 464 & -17 \\
\hline$M W_{\text {det,corr }}[\mathrm{g} / \mathrm{mol}]$ & 542 & & M2 & 369 & -47 \\
\hline \multirow[t]{2}{*}{$M W_{\text {det }}[\mathrm{g} / \mathrm{mol}]$} & 446 & & M1 & 304 & -78 \\
\hline & Signal & $\mathrm{f}$ & Species & $M W_{\text {calc }}[\mathrm{g} / \mathrm{mol}]$ & $M W_{\text {err }}[\%]$ \\
\hline$D_{\mathrm{x}}(\mathrm{f})$ & $8.4910 \mathrm{E}-11$ & & $\mathrm{MgCl}_{2} \cdot \mathrm{D} 1(\mathrm{D} 2) \cdot \mathrm{MgCl}_{2}$ & 655 & 22 \\
\hline $\log D_{\mathrm{x}}(\mathrm{f})$ & $-1.0071 \mathrm{E}+01$ & & D1 & 464 & -10 \\
\hline $\log D_{x, \text { norm }}(f)$ & -9.1137 & & $\mathrm{M} 1 \cdot\left(\mathrm{MgCl}_{2}\right)_{2} \cdot \mathrm{M} 1$ & 943 & 46 \\
\hline$D_{\text {ref }}(\mathrm{PhN})$ & $1.4503 \mathrm{E}-10$ & & $(\mu-\mathrm{Cl} 3)-\mathrm{M} 1 \cdot \mathrm{MgCl}^{(4 \mathrm{THF})}$ & 544 & 6 \\
\hline $\log D_{\text {ref }}(\mathrm{PhN})$ & -9.8385 & & $\mathrm{D} 2$ & 464 & -10 \\
\hline \multirow[t]{4}{*}{$M W_{\text {det }}[\mathrm{g} / \mathrm{mol}]$} & 512 & & M2 & 369 & -39 \\
\hline & & & M1 & 304 & -68 \\
\hline & Signal & $\mathrm{a}$ & too weak in intensity & & $-80^{\circ} \mathrm{C}$ \\
\hline & Signal & $\mathrm{b}$ & Species & $M W_{\text {calc }}[\mathrm{g} / \mathrm{mol}]$ & $M W_{\text {err }}[\%]$ \\
\hline$D_{\mathrm{x}}(\mathrm{b})$ & $7.3170 \mathrm{E}-11$ & & $\mathrm{MgCl}_{2} \cdot \mathrm{D} 1(\mathrm{D} 2) \cdot \mathrm{MgCl}_{2}$ & 655 & 47 \\
\hline $\log D_{\mathrm{x}}(\mathrm{b})$ & $-1.0136 \mathrm{E}+01$ & & D1 & 464 & 25 \\
\hline $\log D_{\mathrm{x}, \text { norm }}(\mathrm{b})$ & -9.0147 & & $\mathrm{M} 1 \cdot\left(\mathrm{MgCl}_{2}\right)_{2} \cdot \mathrm{M} 1$ & 943 & 63 \\
\hline$D_{\text {ref }}(\mathrm{PhN})$ & $9.9497 \mathrm{E}-11$ & & $(\mu-\mathrm{Cl} 3)-\mathrm{M} 1 \cdot \mathrm{MgCl} 2$ & 616 & 44 \\
\hline $\log D_{\text {ref }}(\mathrm{PhN})$ & $-1.0002 \mathrm{E}+01$ & & $\mathrm{D} 2$ & 464 & 25 \\
\hline \multirow[t]{3}{*}{$M W_{\text {det }}[\mathrm{g} / \mathrm{mol}]$} & 346 & & M2 & 369 & 6 \\
\hline & & & M1 & 304 & -14 \\
\hline & Signal & $c+d$ & no signal & & \\
\hline
\end{tabular}




\begin{tabular}{|c|c|c|c|c|c|}
\hline & Signal & e & Species & $M W_{\text {calc }}[\mathrm{g} / \mathrm{mol}]$ & $M W_{\text {err }}[\%]$ \\
\hline$D_{\mathrm{x}}(\mathrm{e})$ & $5.4260 \mathrm{E}-11$ & & $\mathrm{MgCl}_{2} \cdot \mathrm{D} 1(\mathrm{D} 2) \cdot \mathrm{MgCl}_{2}$ & 655 & -7 \\
\hline $\log D_{\mathrm{x}}(\mathrm{e})$ & $-1.0266 \mathrm{E}+01$ & & D1 & 464 & -51 \\
\hline $\log D_{\mathrm{x}, \text { norm }}(\mathrm{e})$ & -9.1445 & & $\mathrm{M} 1 \cdot\left(\mathrm{MgCl}_{2}\right)_{2} \cdot \mathrm{M} 1$ & 943 & 25 \\
\hline$D_{\text {ref }}(\mathrm{PhN})$ & $9.9497 \mathrm{E}-11$ & & $(\mu-\mathrm{Cl} 3)-\mathrm{M} 1 \cdot \mathrm{MgCl}_{2}$ & 616 & -14 \\
\hline $\log D_{\text {ref }}(\mathrm{PhN})$ & $-1.0002 \mathrm{E}+01$ & & $\mathrm{D} 2$ & 464 & -51 \\
\hline$M W_{\text {det,corr }}[\mathrm{g} / \mathrm{mol}]$ & 703 & & M2 & 369 & -91 \\
\hline \multirow{2}{*}{$M W_{\text {det }}[\mathrm{g} / \mathrm{mol}]$} & 578 & & M1 & 304 & -131 \\
\hline & Signal & $\mathrm{f}$ & Species & $M W_{\text {calc }}[\mathrm{g} / \mathrm{mol}]$ & $M W_{\text {err }}[\%]$ \\
\hline$D_{\mathrm{x}}(\mathrm{f})$ & $5.2290 \mathrm{E}-11$ & & $\mathrm{MgCl}_{2} \cdot \mathrm{D} 1(\mathrm{D} 2) \cdot \mathrm{MgCl}_{2}$ & 655 & 6 \\
\hline $\log D_{\mathrm{x}}(\mathrm{f})$ & $-1.0282 \mathrm{E}+01$ & & D1 & 464 & -33 \\
\hline $\log D_{\mathrm{x}, \mathrm{norm}}(\mathrm{f})$ & -9.1606 & & $\mathrm{Ml} \cdot\left(\mathrm{MgCl}_{2}\right)_{2} \cdot \mathrm{M} 1$ & 943 & 35 \\
\hline$D_{\text {ref }}(\mathrm{PhN})$ & $9.9497 \mathrm{E}-11$ & & $(\mu-\mathrm{Cl} 3)-\mathrm{M} 1 \cdot \mathrm{MgCl} 2$ & 616 & 0 \\
\hline $\log D_{\text {ref }}(\mathrm{PhN})$ & $-1.0002 \mathrm{E}+01$ & & D2 & 464 & -33 \\
\hline \multirow[t]{4}{*}{$M W_{\text {det }}[\mathrm{g} / \mathrm{mol}]$} & 616 & & M2 & 369 & -67 \\
\hline & & & M1 & 304 & -102 \\
\hline & Signal & $\mathrm{a}$ & too weak in intensity & & $-90^{\circ} \mathrm{C}$ \\
\hline & Signal & $\mathrm{b}$ & Species & $M W_{\text {calc }}[\mathrm{g} / \mathrm{mol}]$ & $M W_{\mathrm{err}}[\%]$ \\
\hline$D_{\mathrm{x}}(\mathrm{b})$ & $4.5770 \mathrm{E}-11$ & & $\mathrm{MgCl}_{2} \cdot \mathrm{D} 1(\mathrm{D} 2) \cdot \mathrm{MgCl}_{2}$ & 655 & 47 \\
\hline $\log D_{\mathrm{x}}(\mathrm{b})$ & $-1.0339 \mathrm{E}+01$ & & D1 & 464 & 26 \\
\hline $\log D_{\mathrm{x}, \text { norm }}(\mathrm{b})$ & -9.0139 & & $\mathrm{M} 1 \cdot\left(\mathrm{MgCl}_{2}\right)_{2} \cdot \mathrm{M} 1$ & 943 & 63 \\
\hline$D_{\text {ref }}(\mathrm{PhN})$ & $6.2133 \mathrm{E}-11$ & & $(\mu-\mathrm{Cl} 3)-\mathrm{M} 1 \cdot \mathrm{MgCl} 2$ & 616 & 44 \\
\hline $\log D_{\text {ref }}(\mathrm{PhN})$ & $-1.0207 \mathrm{E}+01$ & & D2 & 464 & 26 \\
\hline \multirow[t]{6}{*}{$M W_{\text {det }}[\mathrm{g} / \mathrm{mol}]$} & 345 & & M2 & 369 & 7 \\
\hline & & & M1 & 304 & -13 \\
\hline & Signal & $c+d$ & no signal & & \\
\hline & Signal & $e+f$ & too weak in intensity & & \\
\hline & Signal & $\mathrm{a}$ & too weak in intensity & & $-100^{\circ} \mathrm{C}$ \\
\hline & Signal & $\mathrm{b}$ & Species & $M W_{\text {calc }}[\mathrm{g} / \mathrm{mol}]$ & $M W_{\text {err }}[\%]$ \\
\hline$D_{\mathrm{x}}(\mathrm{b})$ & $2.6840 \mathrm{E}-11$ & & $\mathrm{MgCl}_{2} \cdot \mathrm{D} 1(\mathrm{D} 2) \cdot \mathrm{MgCl}_{2}$ & 655 & 48 \\
\hline $\log D_{\mathrm{x}}(\mathrm{b})$ & $-1.0571 \mathrm{E}+01$ & & $\mathrm{D} 1$ & 464 & 26 \\
\hline $\log D_{\mathrm{x}, \mathrm{norm}}(\mathrm{b})$ & -9.0122 & & $\mathrm{M} 1 \cdot\left(\mathrm{MgCl}_{2}\right)_{2} \cdot \mathrm{M} 1$ & 943 & 64 \\
\hline$D_{\text {ref }}(\mathrm{PhN})$ & $3.6287 \mathrm{E}-11$ & & $(\mu-\mathrm{Cl} 3)-\mathrm{M} 1 \cdot \mathrm{MgCl} 2$ & 616 & 44 \\
\hline $\log D_{\text {ref }}(\mathrm{PhN})$ & $-1.0440 \mathrm{E}+01$ & & D2 & 464 & 26 \\
\hline \multirow[t]{4}{*}{$M W_{\text {det }}[\mathrm{g} / \mathrm{mol}]$} & 342 & & M2 & 369 & 7 \\
\hline & & & M1 & 304 & -13 \\
\hline & Signal & $\mathrm{c}+\mathrm{d}$ & no signal & & \\
\hline & Signal & $e+f$ & too weak in intensity & & \\
\hline
\end{tabular}




\section{${ }^{1} \mathrm{H}$-DOSY-ECC-MW-determination parameters of ${ }^{\mathrm{i}} \mathrm{Pr}_{2} \mathrm{NMgCl} \cdot \mathrm{LiCl} 9$}

A-Table 18. ${ }^{1} \mathrm{H}$-DOSY-ECC-MW-determination of ${ }^{\mathrm{i}} \mathrm{Pr}_{2} \mathrm{NMgCl} \cdot \mathrm{LiCl} 9$ in THF- $d_{8}(0.10 \mathrm{M}) . \mathrm{PhN}(0.02 \mathrm{M})$ was used as internal reference with $\log D_{\text {ref, fix }}(\mathrm{PhN})=-8.8812$. The accuracy of this method is in the range of $M W_{\text {err }}< \pm 9 \%$.

\begin{tabular}{|c|c|c|c|c|c|}
\hline \multirow[t]{2}{*}{${ }^{1} \mathrm{H}-\mathrm{DOSY}$} & & & & & $25^{\circ} \mathrm{C}$ \\
\hline & Signal & $\mathrm{a} 2$ & Species & $M W_{\text {calc }}[\mathrm{g} / \mathrm{mol}]$ & $M W_{\mathrm{err}}[\%]$ \\
\hline$D_{\mathrm{x}}(\mathrm{a} 2)$ & $9.2820 \mathrm{E}-10$ & & $\mathrm{LiCl} \cdot \mathrm{D} 1 \cdot \mathrm{LiCl}$ & 693 & 38 \\
\hline $\log D_{\mathrm{x}}(\mathrm{a} 2)$ & -9.0324 & & D1 & 464 & 8 \\
\hline $\log D_{\mathrm{x}, \text { norm }}(\mathrm{a} 2)$ & -9.0693 & & $\mathrm{LiCl} \cdot \mathrm{D} 2 \cdot \mathrm{LiCl}$ & 693 & 38 \\
\hline$D_{\text {ref }}(\mathrm{PhN})$ & $1.4313 \mathrm{E}-09$ & & D2 & 464 & 8 \\
\hline $\log D_{\text {ref }}(\mathrm{PhN})$ & -8.8443 & & $\mathrm{M} 1 \cdot \mathrm{LiCl}$ & 419 & -3 \\
\hline \multirow[t]{2}{*}{$M W_{\text {det }}[\mathrm{g} / \mathrm{mol}]$} & 429 & & M1 & 304 & -41 \\
\hline & & & M0 & 268 & -60 \\
\hline
\end{tabular}

\begin{tabular}{|c|c|c|c|c|c|}
\hline & Signal & $\mathrm{b} 2$ & too low & intensity & \\
\hline & Signal & $\mathrm{c} 2$ & Species & $M W_{\text {calc }}[\mathrm{g} / \mathrm{mol}]$ & $M W_{\text {err }}[\%]$ \\
\hline$D_{\mathrm{x}}(\mathrm{c} 2)$ & $8.2560 \mathrm{E}-10$ & & $\mathrm{LiCl} \cdot \mathrm{D} 1 \cdot \mathrm{LiCl}$ & 693 & 24 \\
\hline $\log D_{\mathrm{x}}(\mathrm{c} 2)$ & -9.0832 & & D1 & 464 & -13 \\
\hline $\log D_{\mathrm{x}, \text { norm }}(\mathrm{c} 2)$ & -9.1202 & & $\mathrm{LiCl} \cdot \mathrm{D} 2 \cdot \mathrm{LiCl}$ & 693 & 24 \\
\hline$D_{\text {ref }}(\mathrm{PhN})$ & $1.4313 \mathrm{E}-09$ & & $\mathrm{D} 2$ & 464 & -13 \\
\hline $\log D_{\text {ref }}(\mathrm{PhN})$ & -8.8443 & & $\mathrm{M} 1 \cdot \mathrm{LiCl}$ & 419 & -25 \\
\hline \multirow[t]{2}{*}{$M W_{\text {det }}[\mathrm{g} / \mathrm{mol}]$} & 525 & & M1 & 304 & -73 \\
\hline & & & M0 & 268 & -96 \\
\hline
\end{tabular}

\begin{tabular}{|c|c|c|c|c|c|}
\hline & Signal & $\mathrm{a} 2$ & Species & $M W_{\text {calc }}[\mathrm{g} / \mathrm{mol}]$ & $M W_{\mathrm{err}}[\%]$ \\
\hline$D_{\mathrm{x}}(\mathrm{a} 2)$ & $6.1450 \mathrm{E}-10$ & & $\mathrm{LiCl} \cdot \mathrm{D} 1 \cdot \mathrm{LiCl}$ & 693 & 39 \\
\hline $\log D_{\mathrm{x}}(\mathrm{a} 2)$ & -9.2115 & & D1 & 464 & 9 \\
\hline $\log D_{\mathrm{x}, \text { norm }}(\mathrm{a} 2)$ & -9.0647 & & $\mathrm{LiCl} \cdot \mathrm{D} 2 \cdot \mathrm{LiCl}$ & 693 & 39 \\
\hline$D_{\text {ref }}(\mathrm{PhN})$ & $9.3753 \mathrm{E}-10$ & & D2 & 464 & 9 \\
\hline $\log D_{\text {ref }}(\mathrm{PhN})$ & -9.0280 & & $\mathrm{M} 1 \cdot \mathrm{LiCl}$ & 419 & -1 \\
\hline \multirow[t]{3}{*}{$M W_{\text {det }}[\mathrm{g} / \mathrm{mol}]$} & 421 & & M1 & 304 & -39 \\
\hline & & & M0 & 268 & -58 \\
\hline & Signal & b2 & Species & $M W_{\text {calc }}[\mathrm{g} / \mathrm{mol}]$ & $M W_{\mathrm{err}}[\%]$ \\
\hline$D_{\mathrm{x}}(\mathrm{b} 2)$ & $5.5940 \mathrm{E}-10$ & & $\mathrm{LiCl} \cdot \mathrm{D} 1 \cdot \mathrm{LiCl}$ & 693 & 29 \\
\hline $\log D_{\mathrm{x}}(\mathrm{b} 2)$ & -9.2523 & & D1 & 464 & -7 \\
\hline $\log D_{\mathrm{x}, \text { norm }}(\mathrm{b} 2)$ & -9.1055 & & $\mathrm{LiCl} \cdot \mathrm{D} 2 \cdot \mathrm{LiCl}$ & 693 & 29 \\
\hline$D_{\text {ref }}(\mathrm{PhN})$ & $9.3753 \mathrm{E}-10$ & & D2 & 464 & -7 \\
\hline $\log D_{\text {ref }}(\mathrm{PhN})$ & -9.0280 & & $\mathrm{M} 1 \cdot \mathrm{LiCl}$ & 419 & -18 \\
\hline$M W_{\text {det }}[\mathrm{g} / \mathrm{mol}]$ & 495 & & M1 & 304 & -63 \\
\hline & & & 144 & & \\
\hline
\end{tabular}




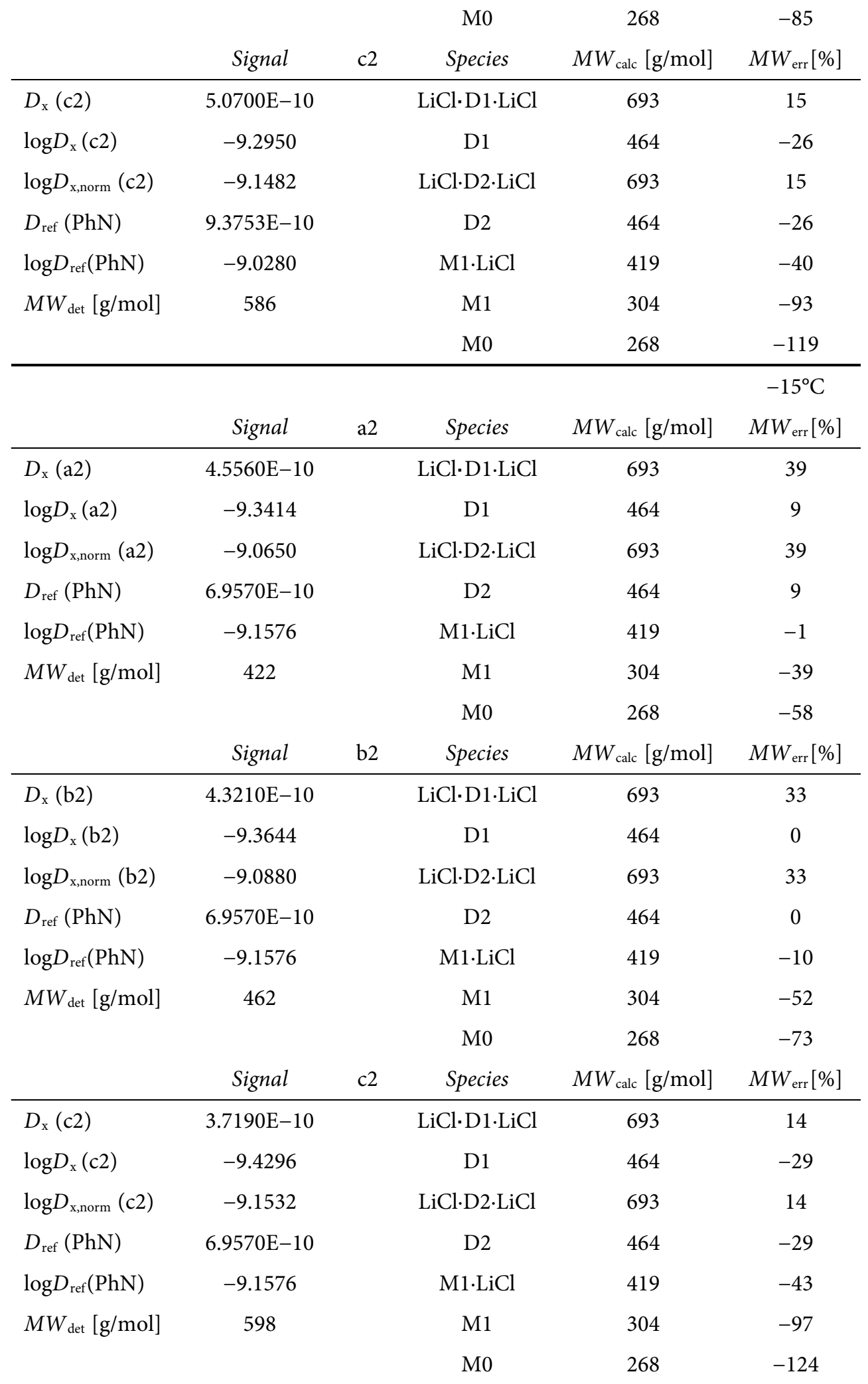




\begin{tabular}{|c|c|c|c|c|c|}
\hline & Signal & $\mathrm{a} 2$ & Species & $M W_{\text {calc }}[\mathrm{g} / \mathrm{mol}]$ & $\begin{array}{c}-40^{\circ} \mathrm{C} \\
M W_{\mathrm{err}}[\%]\end{array}$ \\
\hline$D_{\mathrm{x}}(\mathrm{a} 2)$ & $2.6880 \mathrm{E}-10$ & & $\mathrm{LiCl} \cdot \mathrm{D} 1 \cdot \mathrm{LiCl}$ & 693 & 39 \\
\hline $\log D_{\mathrm{x}}(\mathrm{a} 2)$ & -9.5706 & & D1 & 464 & 9 \\
\hline $\log D_{\mathrm{x}, \text { norm }}(\mathrm{a} 2)$ & -9.0658 & & $\mathrm{LiCl} \cdot \mathrm{D} 2 \cdot \mathrm{LiCl}$ & 693 & 39 \\
\hline$D_{\text {ref }}(\mathrm{PhN})$ & $4.1120 \mathrm{E}-10$ & & D2 & 464 & 9 \\
\hline $\log D_{\text {ref }}(\mathrm{PhN})$ & -9.3859 & & $\mathrm{M} 1 \cdot \mathrm{LiCl}$ & 419 & -1 \\
\hline \multirow[t]{3}{*}{$M W_{\text {det }}[\mathrm{g} / \mathrm{mol}]$} & 423 & & M1 & 304 & -39 \\
\hline & & & M0 & 268 & -58 \\
\hline & Signal & b2 & Species & $M W_{\text {calc }}[\mathrm{g} / \mathrm{mol}]$ & $M W_{\mathrm{err}}[\%]$ \\
\hline$D_{\mathrm{x}}(\mathrm{b} 2)$ & $2.5450 \mathrm{E}-10$ & & $\mathrm{LiCl} \cdot \mathrm{D} 1 \cdot \mathrm{LiCl}$ & 693 & 33 \\
\hline $\log D_{\mathrm{x}}(\mathrm{b} 2)$ & -9.5943 & & D1 & 464 & 0 \\
\hline $\log D_{\mathrm{x}, \text { norm }}(\mathrm{b} 2)$ & -9.0896 & & $\mathrm{LiCl} \cdot \mathrm{D} 2 \cdot \mathrm{LiCl}$ & 693 & 33 \\
\hline$D_{\text {ref }}(\mathrm{PhN})$ & $4.1120 \mathrm{E}-10$ & & $\mathrm{D} 2$ & 464 & 0 \\
\hline $\log D_{\text {ref }}(\mathrm{PhN})$ & -9.3859 & & $\mathrm{M} 1 \cdot \mathrm{LiCl}$ & 419 & -11 \\
\hline \multirow[t]{3}{*}{$M W_{\text {det }}[\mathrm{g} / \mathrm{mol}]$} & 465 & & M1 & 304 & -53 \\
\hline & & & M0 & 268 & -74 \\
\hline & Signal & $\mathrm{c} 2$ & Species & $M W_{\text {calc }}[\mathrm{g} / \mathrm{mol}]$ & $M W_{\mathrm{err}}[\%]$ \\
\hline$D_{\mathrm{x}}(\mathrm{c} 2)$ & $2.1570 \mathrm{E}-10$ & & $\mathrm{LiCl} \cdot \mathrm{D} 1 \cdot \mathrm{LiCl}$ & 693 & 11 \\
\hline $\log D_{\mathrm{x}}(\mathrm{c} 2)$ & -9.6661 & & D1 & 464 & -33 \\
\hline $\log D_{\mathrm{x}, \text { norm }}(\mathrm{c} 2)$ & -9.1614 & & $\mathrm{LiCl} \cdot \mathrm{D} 2 \cdot \mathrm{LiCl}$ & 693 & 11 \\
\hline$D_{\text {ref }}(\mathrm{PhN})$ & $4.1120 \mathrm{E}-10$ & & $\mathrm{D} 2$ & 464 & -33 \\
\hline $\log D_{\text {ref }}(\mathrm{PhN})$ & -9.3859 & & $\mathrm{M} 1 \cdot \mathrm{LiCl}$ & 419 & -48 \\
\hline \multirow[t]{2}{*}{$M W_{\text {det }}[\mathrm{g} / \mathrm{mol}]$} & 618 & & M1 & 304 & -103 \\
\hline & & & M0 & 268 & -131 \\
\hline
\end{tabular}

\begin{tabular}{|c|c|c|c|c|c|}
\hline & Signal & $\mathrm{a} 2$ & Species & $M W_{\text {calc }}[\mathrm{g} / \mathrm{mol}]$ & $M W_{\mathrm{err}}[\%]$ \\
\hline$D_{\mathrm{x}}(\mathrm{a} 2)$ & $1.3470 \mathrm{E}-10$ & & $\mathrm{LiCl} \cdot \mathrm{D} 1 \cdot \mathrm{LiCl}$ & 693 & 37 \\
\hline $\log D_{\mathrm{x}}(\mathrm{a} 2)$ & -9.8706 & & D1 & 464 & 5 \\
\hline $\log D_{\mathrm{x}, \text { norm }}(\mathrm{a} 2)$ & -9.0748 & & $\mathrm{LiCl} \cdot \mathrm{D} 2 \cdot \mathrm{LiCl}$ & 693 & 37 \\
\hline$D_{\text {ref }}(\mathrm{PhN})$ & $2.1037 \mathrm{E}-10$ & & D2 & 464 & 5 \\
\hline $\log D_{\text {ref }}(\mathrm{PhN})$ & -9.6770 & & $\mathrm{M} 1 \cdot \mathrm{LiCl}$ & 419 & -5 \\
\hline \multirow[t]{3}{*}{$M W_{\text {det }}[\mathrm{g} / \mathrm{mol}]$} & 439 & & M1 & 304 & -44 \\
\hline & & & M0 & 268 & -64 \\
\hline & Signal & $\mathrm{b} 2$ & Species & $M W_{\text {calc }}[\mathrm{g} / \mathrm{mol}]$ & $M W_{\mathrm{err}}[\%]$ \\
\hline$D_{\mathrm{x}}(\mathrm{b} 2)$ & $1.3190 \mathrm{E}-10$ & & $\mathrm{LiCl} \cdot \mathrm{D} 1 \cdot \mathrm{LiCl}$ & 693 & 34 \\
\hline $\log D_{\mathrm{x}}(\mathrm{b} 2)$ & -9.8798 & & D1 & 464 & 2 \\
\hline $\log D_{\mathrm{x}, \text { norm }}(\mathrm{b} 2)$ & -9.0839 & & $\mathrm{LiCl} \cdot \mathrm{D} 2 \cdot \mathrm{LiCl}$ & 693 & 34 \\
\hline$D_{\text {ref }}(\mathrm{PhN})$ & $2.1037 \mathrm{E}-10$ & & D2 & 464 & 2 \\
\hline
\end{tabular}




\begin{tabular}{|c|c|c|c|c|c|}
\hline $\log D_{\text {ref }}(\mathrm{PhN})$ & -9.6770 & & $\mathrm{M} 1 \cdot \mathrm{LiCl}$ & 419 & -9 \\
\hline \multirow{3}{*}{$M W_{\text {det }}[\mathrm{g} / \mathrm{mol}]$} & 455 & & M1 & 304 & -50 \\
\hline & & & M0 & 268 & -70 \\
\hline & Signal & $\mathrm{c} 2$ & Species & $M W_{\text {calc }}[\mathrm{g} / \mathrm{mol}]$ & $M W_{\text {err }}[\%]$ \\
\hline$D_{\mathrm{x}}(\mathrm{c} 2)$ & $1.0610 \mathrm{E}-10$ & & $\mathrm{LiCl} \cdot \mathrm{D} 1 \cdot \mathrm{LiCl}$ & 693 & 5 \\
\hline $\log D_{\mathrm{x}}(\mathrm{c} 2)$ & -9.9743 & & D1 & 464 & -42 \\
\hline $\log D_{\mathrm{x}, \mathrm{norm}}(\mathrm{c} 2)$ & -9.1785 & & $\mathrm{LiCl} \cdot \mathrm{D} 2 \cdot \mathrm{LiCl}$ & 693 & 5 \\
\hline$D_{\text {ref }}(\mathrm{PhN})$ & $2.1037 \mathrm{E}-10$ & & D2 & 464 & -42 \\
\hline $\log D_{\text {ref }}(\mathrm{PhN})$ & -9.6770 & & $\mathrm{M} 1 \cdot \mathrm{LiCl}$ & 419 & -58 \\
\hline \multirow[t]{4}{*}{$M W_{\text {det }}[\mathrm{g} / \mathrm{mol}]$} & 661 & & M1 & 304 & -117 \\
\hline & & & M0 & 268 & -147 \\
\hline & & & & & $-70^{\circ} \mathrm{C}$ \\
\hline & Signal & a2 & Species & $M W_{\text {calc }}[\mathrm{g} / \mathrm{mol}]$ & $M W_{\mathrm{err}}[\%]$ \\
\hline$D_{\mathrm{x}}(\mathrm{a} 2)$ & $9.9060 \mathrm{E}-11$ & & $\mathrm{LiCl} \cdot \mathrm{D} 1 \cdot \mathrm{LiCl}$ & 693 & 40 \\
\hline $\log D_{\mathrm{x}}(\mathrm{a} 2)$ & $-1.0004 \mathrm{E}+01$ & & D1 & 464 & 10 \\
\hline $\log D_{\text {x,norm }}(\mathrm{a} 2)$ & -9.0625 & & $\mathrm{LiCl} \cdot \mathrm{D} 2 \cdot \mathrm{LiCl}$ & 693 & 40 \\
\hline$D_{\text {ref }}(\mathrm{PhN})$ & $1.5037 \mathrm{E}-10$ & & D2 & 464 & 10 \\
\hline $\log D_{\text {ref }}(\mathrm{PhN})$ & -9.8228 & & $\mathrm{M} 1 \cdot \mathrm{LiCl}$ & 419 & 0 \\
\hline \multirow[t]{3}{*}{$M W_{\text {det }}[\mathrm{g} / \mathrm{mol}]$} & 418 & & M1 & 304 & -37 \\
\hline & & & M0 & 268 & -56 \\
\hline & Signal & b2 & Species & $M W_{\text {calc }}[\mathrm{g} / \mathrm{mol}]$ & $M W_{\text {err }}[\%]$ \\
\hline$D_{\mathrm{x}}(\mathrm{b} 2)$ & $9.7030 \mathrm{E}-11$ & & $\mathrm{LiCl} \cdot \mathrm{D} 1 \cdot \mathrm{LiCl}$ & 693 & 38 \\
\hline $\log D_{\mathrm{x}}(\mathrm{b} 2)$ & $-1.0013 \mathrm{E}+01$ & & D1 & 464 & 7 \\
\hline $\log D_{\mathrm{x}, \text { norm }}(\mathrm{b} 2)$ & -9.0714 & & $\mathrm{LiCl} \cdot \mathrm{D} 2 \cdot \mathrm{LiCl}$ & 693 & 38 \\
\hline$D_{\text {ref }}(\mathrm{PhN})$ & $1.5037 \mathrm{E}-10$ & & D2 & 464 & 7 \\
\hline $\log D_{\text {ref }}(\mathrm{PhN})$ & -9.8228 & & $\mathrm{M} 1 \cdot \mathrm{LiCl}$ & 419 & -3 \\
\hline \multirow[t]{3}{*}{$M W_{\text {det }}[\mathrm{g} / \mathrm{mol}]$} & 433 & & M1 & 304 & -42 \\
\hline & & & M0 & 268 & -62 \\
\hline & Signal & c2 & Species & $M W_{\text {calc }}[\mathrm{g} / \mathrm{mol}]$ & $M W_{\mathrm{err}}[\%]$ \\
\hline$D_{\mathrm{x}}(\mathrm{c} 2)$ & $7.5890 \mathrm{E}-11$ & & $\mathrm{LiCl} \cdot \mathrm{D} 1 \cdot \mathrm{LiCl}$ & 693 & 5 \\
\hline $\log D_{\mathrm{x}}(\mathrm{c} 2)$ & $-1.0120 \mathrm{E}+01$ & & D1 & 464 & -42 \\
\hline $\log D_{\mathrm{x}, \mathrm{norm}}(\mathrm{c} 2)$ & -9.1782 & & $\mathrm{LiCl} \cdot \mathrm{D} 2 \cdot \mathrm{LiCl}$ & 693 & 5 \\
\hline$D_{\text {ref }}(\mathrm{PhN})$ & $1.5037 \mathrm{E}-10$ & & & D2 & 464 \\
\hline $\log D_{\text {ref }}(\mathrm{PhN})$ & -9.8228 & & $\mathrm{M} 1 \cdot \mathrm{LiCl}$ & 419 & -58 \\
\hline \multirow[t]{2}{*}{$M W_{\text {det }}[\mathrm{g} / \mathrm{mol}]$} & 660 & & M1 & 304 & -117 \\
\hline & & & M0 & 268 & -147 \\
\hline
\end{tabular}




\begin{tabular}{|c|c|c|c|c|c|}
\hline & Signal & $\mathrm{a} 2$ & \multicolumn{2}{|c|}{ too low in intensity } & $-90^{\circ} \mathrm{C}$ \\
\hline & Signal & $\mathrm{b} 2$ & Species & $M W_{\text {calc }}[\mathrm{g} / \mathrm{mol}]$ & $M W_{\text {err }}[\%]$ \\
\hline$D_{\mathrm{x}}(\mathrm{b} 2)$ & $4.2360 \mathrm{E}-11$ & & $\mathrm{LiCl} \cdot \mathrm{D} 1 \cdot \mathrm{LiCl}$ & 693 & 33 \\
\hline $\log D_{\mathrm{x}}(\mathrm{b} 2)$ & $-1.0373 \mathrm{E}+01$ & & D1 & 464 & 0 \\
\hline $\log D_{\mathrm{x}, \text { norm }}(\mathrm{b} 2)$ & -9.0890 & & $\mathrm{LiCl} \cdot \mathrm{D} 2 \cdot \mathrm{LiCl}$ & 693 & 33 \\
\hline$D_{\text {ref }}(\mathrm{PhN})$ & $6.8357 \mathrm{E}-11$ & & D2 & 464 & 0 \\
\hline $\log D_{\text {ref }}(\mathrm{PhN})$ & $-1.0165 \mathrm{E}+01$ & & $\mathrm{M} 1 \cdot \mathrm{LiCl}$ & 419 & -11 \\
\hline \multirow[t]{5}{*}{$M W_{\text {det }}[\mathrm{g} / \mathrm{mol}]$} & 464 & & M1 & 304 & -53 \\
\hline & & & M0 & 268 & -73 \\
\hline & Signal & $\mathrm{c} 2$ & \multicolumn{2}{|c|}{ no signal } & \\
\hline & Signal & $\mathrm{a} 2$ & \multicolumn{2}{|c|}{ too low in intensity } & $-100^{\circ} \mathrm{C}$ \\
\hline & Signal & $\mathrm{b} 2$ & Species & $M W_{\text {calc }}[\mathrm{g} / \mathrm{mol}]$ & $M W_{\text {err }}[\%]$ \\
\hline$D_{\mathrm{x}}(\mathrm{b} 2)$ & $2.9330 \mathrm{E}-11$ & & $\mathrm{LiCl} \cdot \mathrm{D} 1 \cdot \mathrm{LiCl}$ & 693 & 35 \\
\hline $\log D_{\mathrm{x}}(\mathrm{b} 2)$ & $-1.0533 \mathrm{E}+01$ & & D1 & 464 & 3 \\
\hline $\log D_{\mathrm{x}, \text { norm }}(\mathrm{b} 2)$ & -9.0818 & & $\mathrm{LiCl} \cdot \mathrm{D} 2 \cdot \mathrm{LiCl}$ & 693 & 35 \\
\hline$D_{\text {ref }}(\mathrm{PhN})$ & $4.6550 \mathrm{E}-11$ & & D2 & 464 & 3 \\
\hline $\log D_{\text {ref }}(\mathrm{PhN})$ & $-1.0332 \mathrm{E}+01$ & & $\mathrm{M} 1 \cdot \mathrm{LiCl}$ & 419 & -8 \\
\hline \multirow[t]{3}{*}{$M W_{\text {det }}[\mathrm{g} / \mathrm{mol}]$} & 451 & & M1 & 304 & -48 \\
\hline & & & M0 & 268 & -69 \\
\hline & Signal & $\mathrm{b} 2^{\prime}$ & Species & $M W_{\text {calc }}[\mathrm{g} / \mathrm{mol}]$ & $M W_{\text {err }}[\%]$ \\
\hline$D_{\mathrm{x}}\left(\mathrm{b} 2^{\mathrm{c}}\right)$ & $2.3890 \mathrm{E}-11$ & & $\mathrm{LiCl} \cdot \mathrm{D} 1 \cdot \mathrm{LiCl}$ & 693 & 7 \\
\hline $\log D_{\mathrm{x}}\left(\mathrm{b} 2^{\mathrm{c}}\right)$ & $-1.0622 \mathrm{E}+01$ & & D1 & 464 & -38 \\
\hline $\log D_{\mathrm{x}, \text { norm }}\left(\mathrm{b} 2^{\prime}\right)$ & -9.1709 & & $\mathrm{LiCl} \cdot \mathrm{D} 2 \cdot \mathrm{LiCl}$ & 693 & 7 \\
\hline$D_{\text {ref }}(\mathrm{PhN})$ & $4.6550 \mathrm{E}-11$ & & $\mathrm{D} 2$ & 464 & -38 \\
\hline $\log D_{\text {ref }}(\mathrm{PhN})$ & $-1.0332 \mathrm{E}+01$ & & $\mathrm{M} 1 \cdot \mathrm{LiCl}$ & 419 & -53 \\
\hline \multirow[t]{3}{*}{$M W_{\text {det }}[\mathrm{g} / \mathrm{mol}]$} & 641 & & M1 & 304 & -111 \\
\hline & & & M0 & 268 & -140 \\
\hline & Signal & $\mathrm{c} 2$ & \multicolumn{2}{|c|}{ no signal } & \\
\hline
\end{tabular}




\section{${ }^{7}$ Li-DOSY-ECC-MW-determination parameters of ${ }^{\mathrm{i}} \mathrm{Pr}_{2} \mathrm{NMgCl}$ - $\mathrm{LiCl} 9$}

A-Table 19. ${ }^{7} \mathrm{Li}$-DOSY-ECC-MW-determination of ${ }^{\mathrm{i}} \mathrm{Pr}_{2} \mathrm{NMgCl} \cdot \mathrm{LiCl} 9$ in THF- $\mathrm{d}_{8}(0.10 \mathrm{M})$. $\mathrm{PhN}(0.02 \mathrm{M})$ was used as internal reference with $\log D_{\text {ref,fix }}(\mathrm{PhN})=-8.8812$. The accuracy of this method is in the range of $M W_{\text {err }}< \pm 9 \%$.

\begin{tabular}{|c|c|c|c|c|}
\hline${ }^{7} \mathrm{Li}-\mathrm{DOSY}$ & & & & $25^{\circ} \mathrm{C}$ \\
\hline & & Species & $M W_{\text {calc }}[\mathrm{g} / \mathrm{mol}]$ & $M W_{\text {err }}[\%]$ \\
\hline$D(\mathrm{Li})$ & $8.7220 \mathrm{E}-10$ & $\mathrm{LiCl} \cdot \mathrm{D} 1 \cdot \mathrm{LiCl}$ & 693 & 31 \\
\hline $\log D_{\mathrm{x}}(\mathrm{Li})$ & -9.0594 & $\mathrm{LiCl} \cdot \mathrm{D} 2 \cdot \mathrm{LiCl}$ & 693 & 31 \\
\hline $\log D_{\mathrm{x}, \text { norm }}(\mathrm{Li})$ & -9.0963 & $\mathrm{M} 1 \cdot \mathrm{LiCl}$ & 419 & -14 \\
\hline$D_{\text {ref }}(\mathrm{PhN})$ & $1.4313 \mathrm{E}-09$ & LiCl-Dimer & 373 & -28 \\
\hline $\log D_{\text {ref }}(\mathrm{PhN})$ & -8.8443 & $\mathrm{Li}(\mathrm{THF}) 4$ & 295.38 & -62 \\
\hline \multirow[t]{3}{*}{$M W_{\text {det }}$} & 478 & & & \\
\hline & & & & $+0^{\circ} \mathrm{C}$ \\
\hline & & Species & $M W_{\text {calc }}[\mathrm{g} / \mathrm{mol}]$ & $M W_{\mathrm{err}}[\%]$ \\
\hline$D(\mathrm{Li})$ & $5.5920 \mathrm{E}-10$ & $\mathrm{LiCl} \cdot \mathrm{D} 1 \cdot \mathrm{LiCl}$ & 693 & 29 \\
\hline $\log D_{\mathrm{x}}(\mathrm{Li})$ & -9.2524 & $\mathrm{LiCl} \cdot \mathrm{D} 2 \cdot \mathrm{LiCl}$ & 693 & 29 \\
\hline $\log D_{x, \text { norm }}(\mathrm{Li})$ & -9.1056 & $\mathrm{M} 1 \cdot \mathrm{LiCl}$ & 419 & -18 \\
\hline$D_{\text {ref }}(\mathrm{PhN})$ & $9.3753 \mathrm{E}-10$ & LiCl-Dimer & 373 & -33 \\
\hline $\log D_{\text {ref }}(\mathrm{PhN})$ & -9.0280 & $\mathrm{Li}(\mathrm{THF}) 4$ & 295.38 & -68 \\
\hline \multirow[t]{3}{*}{$M W_{\text {det }}$} & 496 & & & \\
\hline & & & & $-15^{\circ} \mathrm{C}$ \\
\hline & & Species & $M W_{\text {calc }}[\mathrm{g} / \mathrm{mol}]$ & $M W_{\mathrm{err}}[\%]$ \\
\hline$D(\mathrm{Li})$ & $4.1750 \mathrm{E}-10$ & $\mathrm{LiCl} \cdot \mathrm{D} 1 \cdot \mathrm{LiCl}$ & 693 & 29 \\
\hline $\log D_{\mathrm{x}}(\mathrm{Li})$ & -9.3793 & $\mathrm{LiCl} \cdot \mathrm{D} 2 \cdot \mathrm{LiCl}$ & 693 & 29 \\
\hline $\log D_{\mathrm{x}, \text { norm }}(\mathrm{Li})$ & -9.1030 & $\mathrm{M} 1 \cdot \mathrm{LiCl}$ & 419 & -17 \\
\hline$D_{\text {ref }}(\mathrm{PhN})$ & $6.9570 \mathrm{E}-10$ & LiCl-Dimer & 373 & -31 \\
\hline $\log D_{\text {ref }}(\mathrm{PhN})$ & -9.1576 & $\operatorname{Li}(\mathrm{THF}) 4$ & 295.38 & -66 \\
\hline \multirow[t]{3}{*}{$M W_{\mathrm{det}}$} & 490 & & & \\
\hline & & & & $-40^{\circ} \mathrm{C}$ \\
\hline & & Species & $M W_{\text {calc }}[\mathrm{g} / \mathrm{mol}]$ & $M W_{\text {err }}[\%]$ \\
\hline$D(\mathrm{Li})$ & $2.5810 \mathrm{E}-10$ & $\mathrm{LiCl} \cdot \mathrm{D} 1 \cdot \mathrm{LiCl}$ & 693 & 34 \\
\hline $\log D_{\mathrm{x}}(\mathrm{Li})$ & -9.5882 & $\mathrm{LiCl} \cdot \mathrm{D} 2 \cdot \mathrm{LiCl}$ & 693 & 34 \\
\hline $\log D_{\mathrm{x}, \text { norm }}(\mathrm{Li})$ & -9.0835 & $\mathrm{M} 1 \cdot \mathrm{LiCl}$ & 419 & -8 \\
\hline$D_{\text {ref }}(\mathrm{PhN})$ & $4.1120 \mathrm{E}-10$ & LiCl-Dimer & 373 & -22 \\
\hline $\log D_{\text {ref }}(\mathrm{PhN})$ & -9.3859 & $\mathrm{Li}(\mathrm{THF}) 4$ & 295.38 & -54 \\
\hline$M W_{\text {det }}$ & 454 & & & \\
\hline
\end{tabular}




\begin{tabular}{|c|c|c|c|c|}
\hline & & & & $-60^{\circ} \mathrm{C}$ \\
\hline & & Species & $M W_{\text {calc }}[\mathrm{g} / \mathrm{mol}]$ & $M W_{\mathrm{err}}[\%]$ \\
\hline$D(\mathrm{Li})$ & $1.4340 \mathrm{E}-10$ & $\mathrm{LiCl} \cdot \mathrm{D} 1 \cdot \mathrm{LiCl}$ & 693 & 43 \\
\hline $\log D_{\mathrm{x}}(\mathrm{Li})$ & -9.8435 & $\mathrm{LiCl} \cdot \mathrm{D} 2 \cdot \mathrm{LiCl}$ & 693 & 43 \\
\hline $\log D_{\mathrm{x}, \text { norm }}(\mathrm{Li})$ & -9.0476 & $\mathrm{M} 1 \cdot \mathrm{LiCl}$ & 419 & 6 \\
\hline$D_{\text {ref }}(\mathrm{PhN})$ & $2.1037 \mathrm{E}-10$ & LiCl-Dimer & 373 & -6 \\
\hline $\log D_{\text {ref }}(\mathrm{PhN})$ & -9.6770 & $\mathrm{Li}(\mathrm{THF}) 4$ & 295 & -33 \\
\hline \multirow{3}{*}{$M W_{\mathrm{det}}$} & 394 & & & \\
\hline & & & & $-70^{\circ} \mathrm{C}$ \\
\hline & & Species & $M W_{\text {calc }}[\mathrm{g} / \mathrm{mol}]$ & $M W_{\mathrm{err}}[\%]$ \\
\hline$D(\mathrm{Li})$ & $1.0740 \mathrm{E}-10$ & $\mathrm{LiCl} \cdot \mathrm{D} 1 \cdot \mathrm{LiCl}$ & 693 & 48 \\
\hline $\log D_{\mathrm{x}}(\mathrm{Li})$ & -9.9690 & $\mathrm{LiCl} \cdot \mathrm{D} 2 \cdot \mathrm{LiCl}$ & 693 & 48 \\
\hline $\log D_{\mathrm{x}, \mathrm{norm}}(\mathrm{Li})$ & -9.0273 & $\mathrm{M} 1 \cdot \mathrm{LiCl}$ & 419 & 13 \\
\hline$D_{\text {ref }}(\mathrm{PhN})$ & $1.5037 \mathrm{E}-10$ & LiCl-Dimer & 373 & 3 \\
\hline $\log D_{\text {ref }}(\mathrm{PhN})$ & -9.8228 & $\mathrm{Li}(\mathrm{THF}) 4$ & 295 & -23 \\
\hline \multirow[t]{3}{*}{$M W_{\text {det }}$} & 364 & & & \\
\hline & & & & $-90^{\circ} \mathrm{C}$ \\
\hline & & Species & $M W_{\text {calc }}[\mathrm{g} / \mathrm{mol}]$ & $M W_{\mathrm{err}}[\%]$ \\
\hline$D(\mathrm{Li})$ & $4.7090 \mathrm{E}-11$ & $\mathrm{LiCl} \cdot \mathrm{D} 1 \cdot \mathrm{LiCl}$ & 693 & 44 \\
\hline $\log D_{\mathrm{x}}(\mathrm{Li})$ & $-1.0327 \mathrm{E}+01$ & $\mathrm{LiCl} \cdot \mathrm{D} 2 \cdot \mathrm{LiCl}$ & 693 & 44 \\
\hline $\log D_{\mathrm{x}, \text { norm }}(\mathrm{Li})$ & -9.0431 & $\mathrm{M} 1 \cdot \mathrm{LiCl}$ & 419 & 8 \\
\hline$D_{\text {ref }}(\mathrm{PhN})$ & $6.8357 \mathrm{E}-11$ & LiCl-Dimer & 373 & -4 \\
\hline $\log D_{\text {ref }}(\mathrm{PhN})$ & $-1.0165 \mathrm{E}+01$ & $\mathrm{Li}(\mathrm{THF}) 4$ & 295 & -31 \\
\hline \multirow[t]{3}{*}{$M W_{\text {det }}$} & 387 & & & \\
\hline & & & & $-100^{\circ} \mathrm{C}$ \\
\hline & & Species & $M W_{\text {calc }}[\mathrm{g} / \mathrm{mol}]$ & $M W_{\mathrm{err}}[\%]$ \\
\hline$D(\mathrm{Li})$ & $3.2170 \mathrm{E}-11$ & $\mathrm{LiCl} \cdot \mathrm{D} 1 \cdot \mathrm{LiCl}$ & 693 & 44 \\
\hline $\log D_{\mathrm{x}}(\mathrm{Li})$ & $-1.0493 \mathrm{E}+01$ & $\mathrm{LiCl} \cdot \mathrm{D} 2 \cdot \mathrm{LiCl}$ & 693 & 44 \\
\hline $\log D_{x, \text { norm }}(\mathrm{Li})$ & -9.0417 & $\mathrm{M} 1 \cdot \mathrm{LiCl}$ & 419 & 8 \\
\hline$D_{\text {ref }}(\mathrm{PhN})$ & $4.6550 \mathrm{E}-11$ & LiCl-Dimer & 373 & -3 \\
\hline $\log D_{\text {ref }}(\mathrm{PhN})$ & $-1.0332 \mathrm{E}+01$ & $\mathrm{Li}(\mathrm{THF}) 4$ & 295.38 & -30 \\
\hline$M W_{\text {det }}$ & 385 & & & \\
\hline
\end{tabular}


A-Table 20. DOSY-ECC-MW-determination of anhydrous LiCl in THF- $d_{8} \quad(0.015 \mathrm{M})$. 2,2,3,3tetramethylbutane $(\mathrm{TMB})$ was used as internal reference with $\log D_{\text {ref,fix }}(\mathrm{TMB})=-8.7749$.

\begin{tabular}{lcccccc}
\hline & $25^{\circ} \mathrm{C}$ & $0^{\circ} \mathrm{C}$ & $-25^{\circ} \mathrm{C}$ & $-50^{\circ} \mathrm{C}$ & $-75^{\circ} \mathrm{C}$ & $a v$. \\
\hline & & & & & & \\
$D_{\text {ref }}(\mathrm{TMB})$ & $2.003 \mathrm{E}-09$ & $1.319 \mathrm{E}-09$ & $8.821 \mathrm{E}-10$ & $4.806 \mathrm{E}-10$ & $2.299 \mathrm{E}-10$ & \\
$D_{\mathrm{x}}(\mathrm{Li})$ & $1.102 \mathrm{E}-09$ & $7.318 \mathrm{E}-10$ & $4.790 \mathrm{E}-10$ & $2.631 \mathrm{E}-10$ & $1.204 \mathrm{E}-10$ & \\
$M W_{\text {calc }}[\mathrm{g} / \mathrm{mol}]$ & 373 & 373 & 373 & 373 & 373 & 373 \\
$M W_{\text {det }}[\mathrm{g} / \mathrm{mol}]$ & 374 & 369 & 382 & 377 & 401 & 381 \\
$M W_{\text {err }}[\%]$ & 0 & 1 & -2 & -1 & -7 & -2
\end{tabular}

Aggregate M1·( $\left.\mathbf{M g C l}_{2}\right)_{2} \cdot \mathbf{M 1}\left(M W_{\text {calc }}=943 \mathrm{~g} / \mathrm{mol}\right)$ show an increased van der Waals value of $M D_{\mathrm{W}}=6.26 \cdot 10^{29}$ (A-Table 21). This aggregate would be underestimated in MW by approximately $10 \%, \Delta M W=94 \mathrm{~g} / \mathrm{mol}$. However, the highest MW we could observe was $M W_{\text {det }}=578 \mathrm{~g} / \mathrm{mol}$ so aggregate $\mathbf{M 1} \cdot\left(\mathbf{M g C l}_{2}\right)_{2} \cdot \mathbf{M 1}$ can be excluded to be present in solution of 7 .

A-Table 21. Calculation of the molar van der Waals density $M D_{w}$ of all ${ }^{i} \mathrm{Pr}_{2} \mathrm{NMgCl} 7$ species.

\begin{tabular}{|c|c|c|c|c|}
\hline Species & & Formula & $\begin{array}{l}M W_{\text {calc }} \\
{[\mathrm{g} / \mathrm{mol}]}\end{array}$ & $\begin{array}{c}M D_{\mathrm{w}} / 10^{29} \\
{\left[\mathrm{~g} /\left(\mathrm{mol} \cdot \mathrm{m}^{3}\right)\right]}\end{array}$ \\
\hline & $\mathrm{M1} \cdot\left(\mathrm{MgCl}_{2}\right)_{2} \cdot \mathrm{M} 1$ & $\mathrm{C} 36 \mathrm{H} 76 \mathrm{Cl} 6 \mathrm{Mg} 4 \mathrm{~N} 2 \mathrm{O} 6$ & 943 & 6.26 \\
\hline e1 & $\mathrm{MgCl}_{2} \cdot \mathrm{D} 1(\mathrm{D} 2) \cdot \mathrm{MgCl}_{2}$ & $\mathrm{C} 20 \mathrm{H} 44 \mathrm{Cl} 6 \mathrm{Mg} 4 \mathrm{~N} 2 \mathrm{O} 2$ & 655 & 6.98 \\
\hline $\mathrm{f} 1$ & $(\mu-\mathrm{Cl} 3)-\mathrm{M} 1 \cdot \mathrm{MgCl} 2$ & C26H54Cl3Mg2NO5 & 616 & 5.95 \\
\hline $\mathrm{d} 1$ & D1 & $\mathrm{C} 20 \mathrm{H} 44 \mathrm{Cl} 2 \mathrm{Mg} 2 \mathrm{~N} 2 \mathrm{O} 2$ & 464 & 5.76 \\
\hline $\mathrm{c} 1$ & D2 & $\mathrm{C} 20 \mathrm{H} 44 \mathrm{Cl} 2 \mathrm{Mg} 2 \mathrm{~N} 2 \mathrm{O} 2$ & 464 & 5.76 \\
\hline b1 & M2 & $\mathrm{C} 20 \mathrm{H} 44 \mathrm{MgN} 2 \mathrm{O} 2$ & 369 & 4.99 \\
\hline a1 & M1 & $\mathrm{C} 14 \mathrm{H} 30 \mathrm{ClMgNO} 2$ & 304 & 5.58 \\
\hline
\end{tabular}

Compound $\mathbf{M g C l}_{2} \cdot \mathbf{D 1}(\mathbf{D 2}) \cdot \mathbf{M g C l}_{2}\left(M W_{\text {calc }}=655 \mathrm{~g} / \mathrm{mol}\right)$ has a very huge van der Waals density. This is why this aggregate would be highly underestimated in MW by approximately $20 \%, \Delta M W=131 \mathrm{~g} / \mathrm{mol}$. For species e1 a $\mathrm{MW}$ of $M W_{\mathrm{det}}=578 \mathrm{~g} / \mathrm{mol}$ was measured that is in the $20 \%$ region of compound $\mathbf{M g C l}_{2} \cdot \mathbf{D 1}(\mathbf{D} 2) \cdot \mathbf{M g C l}_{2}$. This is why a density correction was done (see chapter 2.1.7). The results are shown in A-Table 22: 
A-Table 22. Density correction of species e1.

\begin{tabular}{cccccc}
\hline Species e1 & $T\left[{ }^{\circ} \mathrm{C}\right]$ & $\begin{array}{c}M W_{\text {calc }} \\
{[\mathrm{g} / \mathrm{mol}]}\end{array}$ & $\begin{array}{c}M W_{\text {det }} \\
{[\mathrm{g} / \mathrm{mol}]}\end{array}$ & $X_{\text {corr }}$ & $\begin{array}{c}M W_{\text {det,corr }} \\
{[\mathrm{g} / \mathrm{mol}]}\end{array}$ \\
\hline $\mathbf{M g C l}_{2} \cdot \mathbf{D 1}(\mathbf{D 2}) \cdot \mathbf{M g C l}_{2}$ & -80 & 655 & 578 & 1.22 & 703 \\
$M D_{\mathrm{W}}=6.98 \cdot 10^{29}$ & -70 & 655 & 446 & 1.22 & 542 \\
& -60 & 655 & 409 & 1.22 & 497
\end{tabular}

A-Table 23. Calculation of the molar van der Waals density $M D_{w}$ of all ${ }^{i} \operatorname{Pr}_{2} \mathrm{NMgCl} \cdot \mathrm{LiCl} 9$ species.

\begin{tabular}{|c|c|c|c|c|}
\hline Species & & Formula & $\begin{array}{l}M W_{\text {calc }} \\
{[\mathrm{g} / \mathrm{mol}]}\end{array}$ & $\begin{array}{c}M D_{\mathrm{w}} / 10^{29} \\
{\left[\mathrm{~g} /\left(\mathrm{mol} \cdot \mathrm{m}^{3}\right)\right]}\end{array}$ \\
\hline$b^{\prime}$ & $\mathrm{M1} \cdot(\mathrm{LiCl})_{2} \cdot \mathrm{M} 1$ & $\mathrm{C} 28 \mathrm{H} 60 \mathrm{Cl} 4 \mathrm{Li} 2 \mathrm{Mg} 2 \mathrm{~N} 2 \mathrm{O} 4$ & 693 & 5.85 \\
\hline \multirow[t]{2}{*}{ c } & $\mathrm{LiCl} \cdot \mathrm{D} 1 \cdot \mathrm{LiCl}$ & $\mathrm{C} 28 \mathrm{H} 60 \mathrm{Cl} 4 \mathrm{Li} 2 \mathrm{Mg} 2 \mathrm{~N} 2 \mathrm{O} 4$ & 693 & 5.85 \\
\hline & D1 & $\mathrm{C} 20 \mathrm{H} 44 \mathrm{Cl} 2 \mathrm{Mg} 2 \mathrm{~N} 2 \mathrm{O} 2$ & 464 & 5.76 \\
\hline $\mathrm{b}$ & D2 & $\mathrm{C} 20 \mathrm{H} 44 \mathrm{Cl} 2 \mathrm{Mg} 2 \mathrm{~N} 2 \mathrm{O} 2$ & 464 & 5.76 \\
\hline a & $\mathrm{M1} \cdot \mathrm{LiCl}$ & $\mathrm{C} 18 \mathrm{H} 38 \mathrm{Cl} 2 \mathrm{LiMgNO} 3$ & 418 & 5.70 \\
\hline \multirow[t]{4}{*}{$\mathrm{Li}$} & Li1 & $\mathrm{C} 16 \mathrm{H} 32 \mathrm{Cl} 2 \mathrm{Li} 2 \mathrm{O} 4$ & 373 & 5.64 \\
\hline & M1 & $\mathrm{C} 14 \mathrm{H} 30 \mathrm{ClMgNO} 2$ & 304 & 5.58 \\
\hline & Li2 & $\mathrm{C} 16 \mathrm{H} 32 \mathrm{LiO} 4$ & 295 & 4.99 \\
\hline & M0 & $\mathrm{C} 10 \mathrm{H} 22 \mathrm{Cl} 2 \mathrm{MgNO}$ & 268 & 6.30 \\
\hline
\end{tabular}

In the case of ${ }^{i} \mathrm{Pr}_{2} \mathrm{NMgCl} \cdot \mathrm{LiCl} 9$ all van der Waals densities are in the desired range (ATable 23) of $M D_{\mathrm{W}}=4.3 \cdot 10^{29} \mathrm{~g} /\left(\mathrm{mol} \cdot \mathrm{m}^{3}\right)$ and $M D_{\mathrm{W}}=5.9 \cdot 10^{29} \mathrm{~g} /\left(\mathrm{mol} \cdot \mathrm{m}^{3}\right)$. Only species M0 $\left(M W_{\text {calc }}=268 \mathrm{~g} / \mathrm{mol}\right)$ would be underestimated in MW by approximately $20 \%$, $\Delta M W=54 \mathrm{~g} / \mathrm{mol}$. However, the smallest $M W$ we could observe was $M W_{\text {det }}=364 \mathrm{~g} / \mathrm{mol}$, so species M0 can be excluded to be present in solution of $\mathbf{9}$. 


\section{Optimized Structures from theoretical calculations}

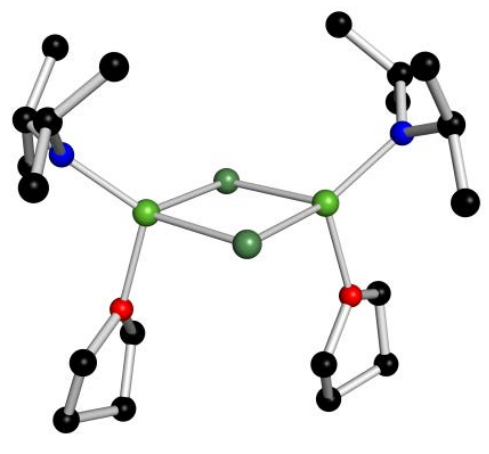

D2-cis

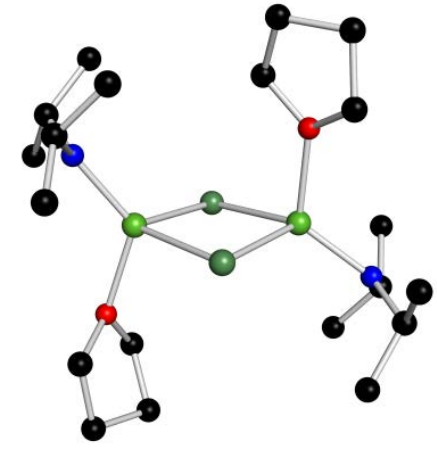

D2-trans

A-Figure 12. B3LYP-D3/def2-SVP optimized structures of D2 (cis and trans).

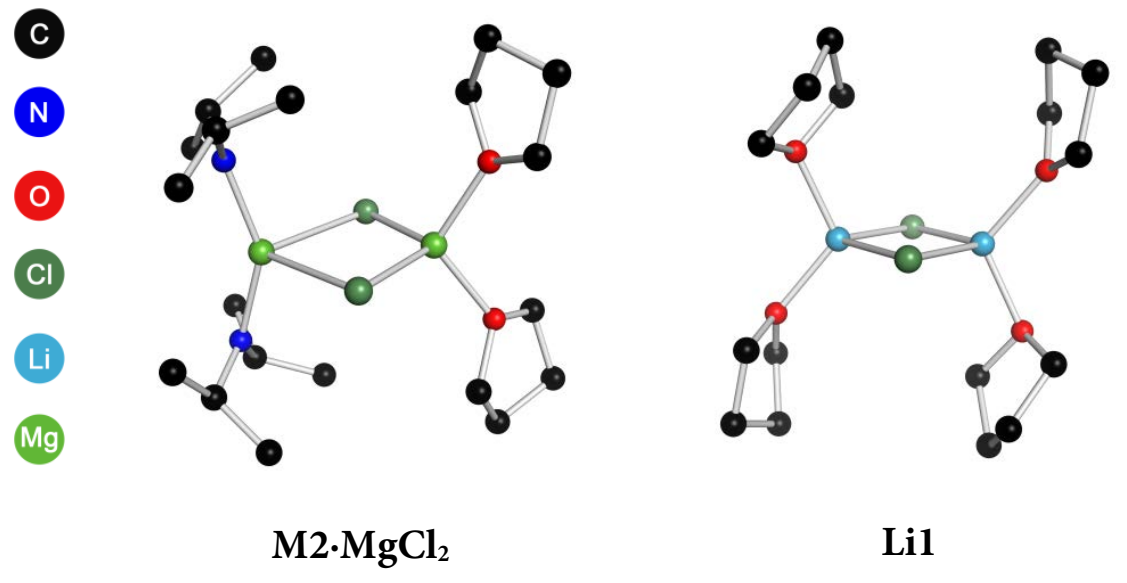

A-Figure 13. B3LYP-D3/def2-SVP optimized structures of $\mathbf{M 2} \cdot \mathbf{M g C l}_{\mathbf{2}}$ and $\mathbf{L i 1}$. 


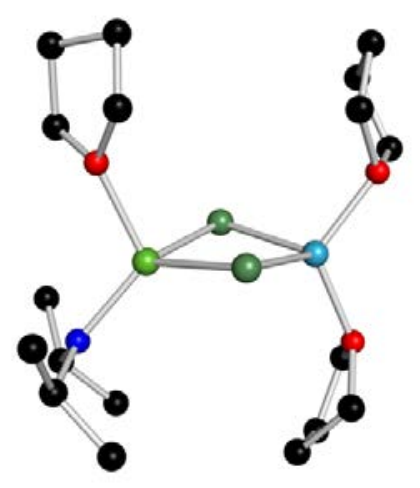

\section{M1·LiCl}

A-Figure 14. B3LYP-D3/def2-SVP optimized structure of M1·LiCl.

C

(N)

(O)

(CI)

(Li)

Mg

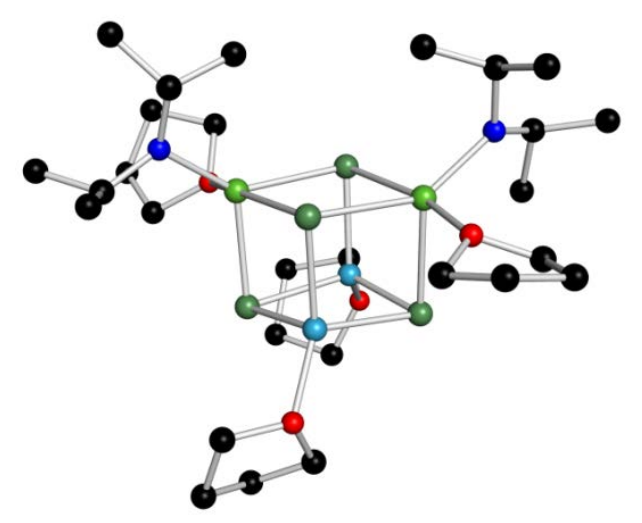

Li1·D2-cube(A)

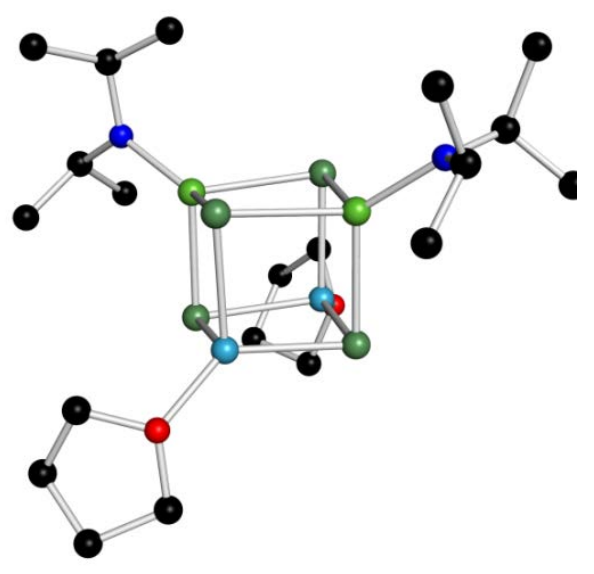

Li1·D2-cube(C)

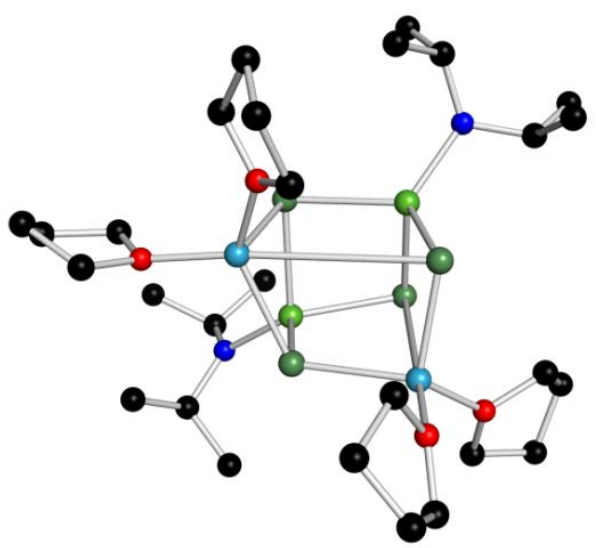

Li1·D2-cube(B)

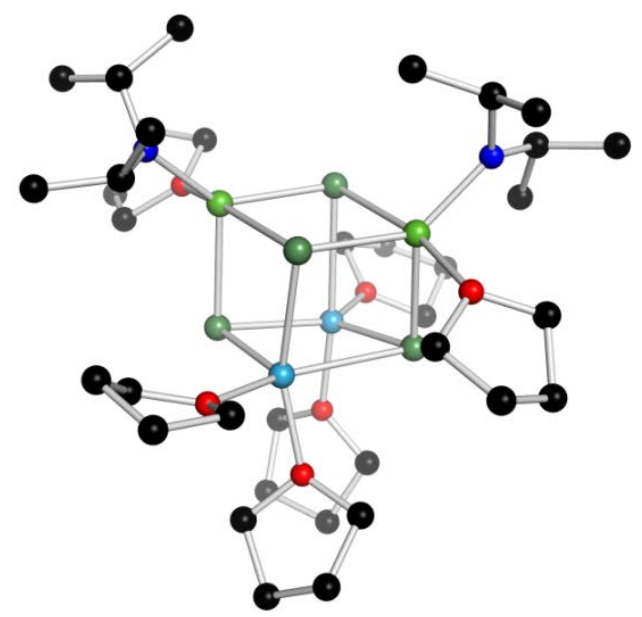

Li1·D2-cube(D)

A-Figure 15. B3LYP-D3/def2-SVP optimized structures of Li1·D2-cube(A-D). 


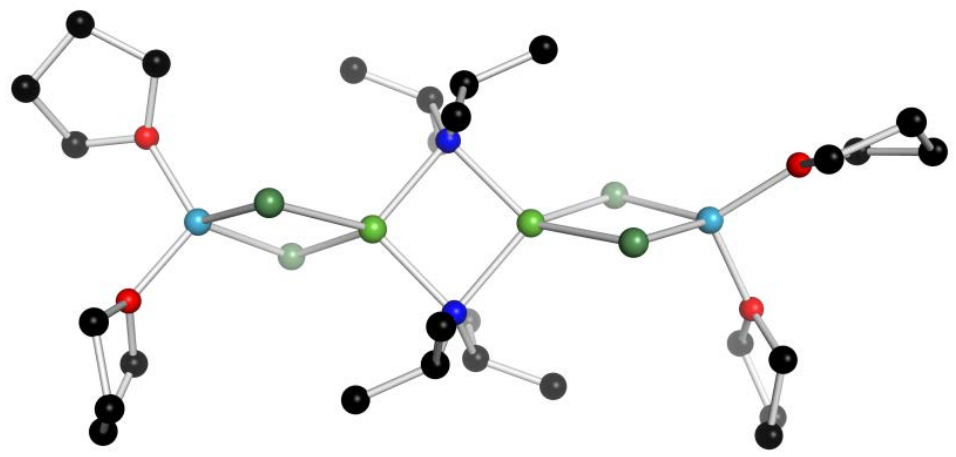

LiCl·D 1·LiCl

A-Figure 16. B3LYP-D3/def2-SVP optimized structures of LiCl·D1·LiCl.
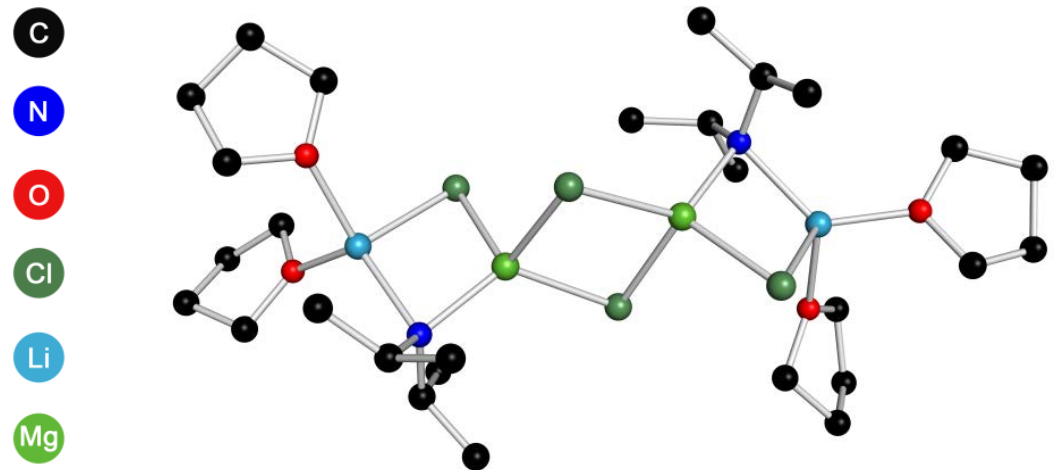

\section{$\mathrm{LiCl} \cdot \mathrm{D} 2^{*} \cdot \mathrm{LiCl}$}

A-Figure 17. B3LYP-D3/def2-SVP optimized structures of LiCl·D2* ${ }^{\star} \mathbf{L i C l}$.

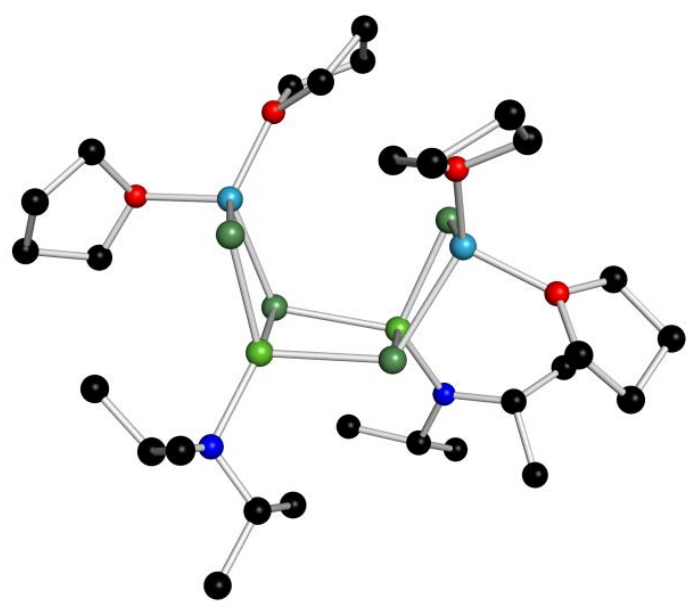

LiCl-D2·LiCl-boat

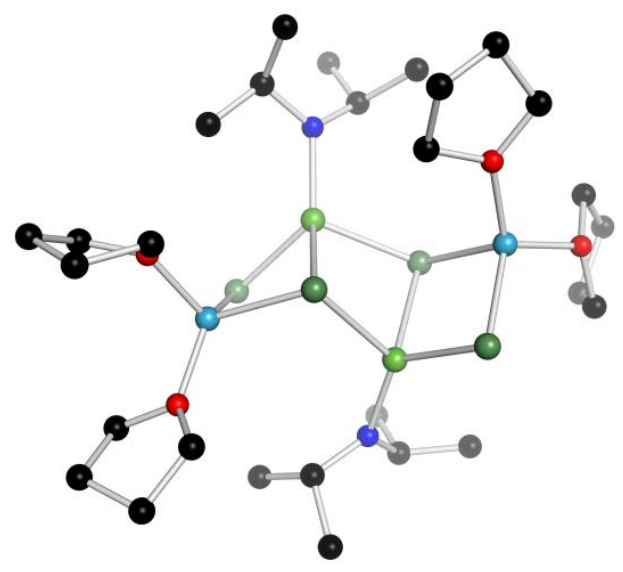

LiCl-D2.LiCl-ladder

A-Figure 18. B3LYP-D3/def2-SVP optimized structures of LiCl-D2·LiCl (boat and ladder). 


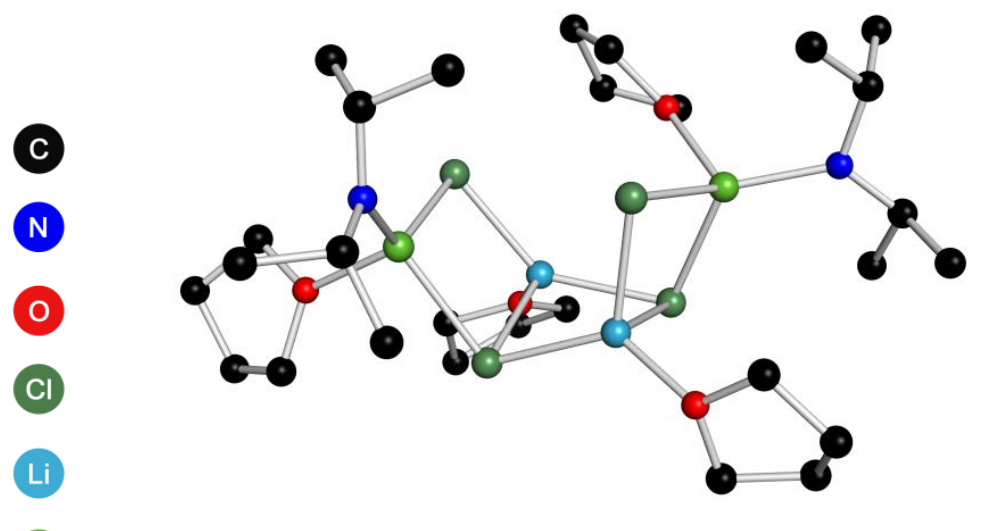

Mg

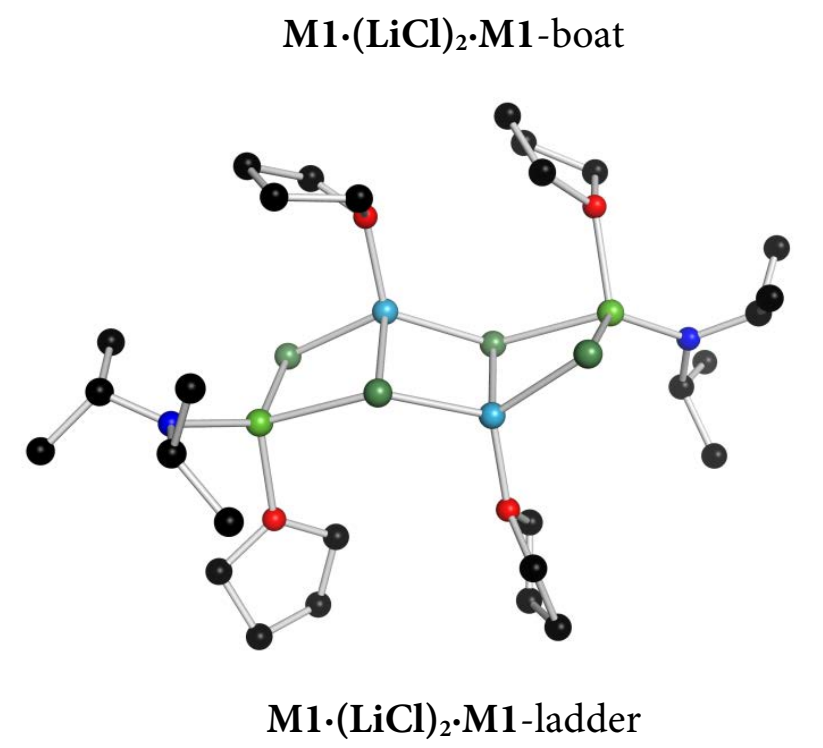

A-Figure 19. B3LYP-D3/def2-SVP optimized structures of M1·(LiCl) ${ }_{2} \cdot \mathrm{M} 1$ (boat and ladder). 


\section{Free Enthalpies of computed species}

A-Table 24. Relative free enthalpies $\Delta G$ in $\mathrm{kJ} / \mathrm{mol}$. (left: D2-cis and right Cube D was used as reference)

\begin{tabular}{|c|c|c|c|c|c|c|c|}
\hline$T\left[{ }^{\circ} \mathrm{C}\right]$ & D2-cis & D2-trans & $\mathrm{M} 2 \cdot \mathrm{MgCl}_{2}$ & Cube A & Cube B & Cube C & Cube D \\
\hline-90 & 0 & 5.5 & 53.7 & 10.6 & 20.4 & 51.5 & 0.0 \\
\hline-85 & 0 & 5.6 & 53.7 & 9.1 & 18.9 & 48.4 & 0.0 \\
\hline-80 & 0 & 5.7 & 53.6 & 7.5 & 17.3 & 45.2 & 0.0 \\
\hline-75 & 0 & 5.7 & 53.6 & 5.9 & 15.7 & 42.1 & 0.0 \\
\hline-70 & 0 & 5.8 & 53.6 & 4.4 & 14.2 & 39.1 & 0.0 \\
\hline-65 & 0 & 5.9 & 53.6 & 2.8 & 12.6 & 36.0 & 0.0 \\
\hline-60 & 0 & 6.0 & 53.6 & 1.3 & 11.1 & 32.9 & 0.0 \\
\hline-55 & 0 & 6.0 & 53.6 & 0.0 & 9.8 & 30.1 & 0.3 \\
\hline-50 & 0 & 6.1 & 53.6 & 0.0 & 9.8 & 28.6 & 1.8 \\
\hline-45 & 0 & 6.2 & 53.5 & 0.0 & 9.8 & 27.0 & 3.4 \\
\hline-40 & 0 & 6.3 & 53.5 & 0.0 & 9.8 & 25.5 & 4.9 \\
\hline-35 & 0 & 6.3 & 53.5 & 0.0 & 9.8 & 24.0 & 6.5 \\
\hline-30 & 0 & 6.4 & 53.5 & 0.0 & 9.8 & 22.5 & 8.0 \\
\hline-25 & 0 & 6.5 & 53.4 & 0.0 & 9.8 & 21.0 & 9.6 \\
\hline-20 & 0 & 6.6 & 53.4 & 0.0 & 9.9 & 19.5 & 11.1 \\
\hline-15 & 0 & 6.7 & 53.4 & 0.0 & 9.9 & 18.0 & 12.7 \\
\hline-10 & 0 & 6.7 & 53.4 & 0.0 & 9.9 & 16.5 & 14.2 \\
\hline-5 & 0 & 6.8 & 53.3 & 0.0 & 9.9 & 15.0 & 15.7 \\
\hline 0 & 0 & 6.9 & 53.3 & 0.0 & 9.9 & 13.5 & 17.3 \\
\hline 5 & 0 & 7.0 & 53.3 & 0.0 & 9.9 & 12.0 & 18.8 \\
\hline 10 & 0 & 7.1 & 53.3 & 0.0 & 10.0 & 10.5 & 20.3 \\
\hline 15 & 0 & 7.1 & 53.2 & 0.0 & 10.0 & 9.1 & 21.9 \\
\hline 20 & 0 & 7.2 & 53.2 & 0.0 & 10.0 & 7.6 & 23.4 \\
\hline 25 & 0 & 7.3 & 53.2 & 0.0 & 10.0 & 6.1 & 24.9 \\
\hline
\end{tabular}


A-Table 25. Relative free enthalpies $\Delta G$ for mixed dimers of $\mathrm{LiCl}$ and ${ }^{i} \operatorname{Pr}_{2} \mathrm{NMgCl}$ in $\mathrm{kJ} / \mathrm{mol}$, with $\mathrm{LiCl} \cdot \mathrm{D} 1 \cdot \mathrm{LiCl}$ as reference.

\begin{tabular}{|c|c|c|c|c|c|}
\hline$T\left[{ }^{\circ} \mathrm{C}\right]$ & $\begin{array}{c}\mathrm{LiCl} \cdot \mathrm{D} 2 \cdot \mathrm{LiCl}- \\
\text { boat }\end{array}$ & $\begin{array}{c}\mathrm{LiCl} \cdot \mathrm{D} 2 \cdot \mathrm{LiCl}- \\
\text { ladder }\end{array}$ & $\begin{array}{l}M 1 \cdot(\mathrm{LiCl})_{2} \cdot \mathrm{M}- \\
\text { boat }\end{array}$ & $\begin{array}{c}M 1 \cdot(\mathrm{LiCl})_{2} \cdot \mathrm{M1}- \\
\text { ladder }\end{array}$ & $\mathrm{LiCl} \cdot \mathrm{D} 1 \cdot \mathrm{LiCl}$ \\
\hline-90 & 95.7 & 97.6 & 55.2 & 57.8 & 0.0 \\
\hline-85 & 95.7 & 97.5 & 55.1 & 57.8 & 0.0 \\
\hline-80 & 95.7 & 97.5 & 55.1 & 57.7 & 0.0 \\
\hline-75 & 95.7 & 97.4 & 55.0 & 57.6 & 0.0 \\
\hline-70 & 95.7 & 97.3 & 54.9 & 57.6 & 0.0 \\
\hline-65 & 95.7 & 97.2 & 54.9 & 57.5 & 0.0 \\
\hline-60 & 95.7 & 97.2 & 54.8 & 57.5 & 0.0 \\
\hline-55 & 95.7 & 97.1 & 54.8 & 57.4 & 0.0 \\
\hline-50 & 95.7 & 97.0 & 54.7 & 57.3 & 0.0 \\
\hline-45 & 95.7 & 97.0 & 54.6 & 57.3 & 0.0 \\
\hline-40 & 95.8 & 96.9 & 54.6 & 57.2 & 0.0 \\
\hline-35 & 95.8 & 96.8 & 54.5 & 57.1 & 0.0 \\
\hline-30 & 95.8 & 96.8 & 54.5 & 57.1 & 0.0 \\
\hline-25 & 95.8 & 96.7 & 54.4 & 57.0 & 0.0 \\
\hline-20 & 95.8 & 96.6 & 54.3 & 56.9 & 0.0 \\
\hline-15 & 95.8 & 96.6 & 54.3 & 56.9 & 0.0 \\
\hline-10 & 95.8 & 96.5 & 54.2 & 56.8 & 0.0 \\
\hline-5 & 95.9 & 96.4 & 54.2 & 56.7 & 0.0 \\
\hline 0 & 95.9 & 96.3 & 54.1 & 56.6 & 0.0 \\
\hline 5 & 95.9 & 96.3 & 54.0 & 56.6 & 0.0 \\
\hline 10 & 95.9 & 96.2 & 54.0 & 56.5 & 0.0 \\
\hline 15 & 95.9 & 96.1 & 53.9 & 56.4 & 0.0 \\
\hline 20 & 96.0 & 96.1 & 53.9 & 56.4 & 0.0 \\
\hline 25 & 96.0 & 96.0 & 53.8 & 56.3 & 0.0 \\
\hline
\end{tabular}


Free Reaction enthalpies for the reaction of D2 and Li1

A-Table 26. Free Reaction enthalpies for the reaction of $\mathrm{D} 2$ and Li1 in $\mathrm{kJ} / \mathrm{mol}$.

\begin{tabular}{|c|c|c|c|c|}
\hline$T\left[{ }^{\circ} \mathrm{C}\right]$ & CUBE A & CUBE B & CUBE C & CUBE D \\
\hline-90 & 21.4 & 31.2 & 62.2 & 10.7 \\
\hline-85 & 20.9 & 30.7 & 60.2 & 11.9 \\
\hline-80 & 20.5 & 30.3 & 58.3 & 13.0 \\
\hline-75 & 20.1 & 29.9 & 56.3 & 14.1 \\
\hline-70 & 19.6 & 29.4 & 54.3 & 15.3 \\
\hline-65 & 19.2 & 29.0 & 52.3 & 16.4 \\
\hline-60 & 18.8 & 28.6 & 50.4 & 17.5 \\
\hline-55 & 18.3 & 28.1 & 48.4 & 18.6 \\
\hline-50 & 17.9 & 27.7 & 46.5 & 19.7 \\
\hline-45 & 17.5 & 27.3 & 44.5 & 20.9 \\
\hline-40 & 17.1 & 26.9 & 42.6 & 22.0 \\
\hline-35 & 16.6 & 26.4 & 40.6 & 23.1 \\
\hline-30 & 16.2 & 26.0 & 38.7 & 24.2 \\
\hline-25 & 15.8 & 25.6 & 36.8 & 25.3 \\
\hline-20 & 15.3 & 25.2 & 34.8 & 26.5 \\
\hline-15 & 14.9 & 24.8 & 32.9 & 27.6 \\
\hline-10 & 14.5 & 24.4 & 31.0 & 28.7 \\
\hline-5 & 14.1 & 24.0 & 29.1 & 29.8 \\
\hline 0 & 13.6 & 23.5 & 27.1 & 30.9 \\
\hline 5 & 13.2 & 23.1 & 25.2 & 32.0 \\
\hline 10 & 12.8 & 22.7 & 23.3 & 33.1 \\
\hline 15 & 12.4 & 22.3 & 21.4 & 34.2 \\
\hline 20 & 11.9 & 21.9 & 19.5 & 35.3 \\
\hline 25 & 11.5 & 21.5 & 17.6 & 36.4 \\
\hline
\end{tabular}


A-Table 27. Free Reaction enthalpies for the reaction of D2 and Lil in $\mathrm{kJ} / \mathrm{mol}$.

\begin{tabular}{|c|c|c|c|c|c|}
\hline$T\left[{ }^{\circ} \mathrm{C}\right]$ & $\begin{array}{c}\mathrm{LiCl} \cdot \mathrm{D} 2 \cdot \mathrm{LiCl}- \\
\text { boat }\end{array}$ & $\begin{array}{c}\mathrm{LiCl} \cdot \mathrm{D} 2 \cdot \mathrm{LiCl}- \\
\text { ladder }\end{array}$ & $\begin{array}{c}M 1 \cdot(\mathrm{LiCl})_{2} \cdot M 1- \\
\text { boat }\end{array}$ & $\begin{array}{c}M 1 \cdot(\mathrm{LiCl})_{2} \cdot \mathrm{M1}- \\
\text { ladder }\end{array}$ & $\mathrm{LiCl} \cdot \mathrm{D} 1 \cdot \mathrm{LiCl}$ \\
\hline-90 & 37.1 & 39.1 & -3.3 & -0.7 & -58.5 \\
\hline-85 & 36.7 & 38.6 & -3.8 & -1.2 & -58.9 \\
\hline-80 & 36.3 & 38.1 & -4.3 & -1.7 & -59.4 \\
\hline-75 & 35.9 & 37.6 & -4.8 & -2.2 & -59.8 \\
\hline-70 & 35.5 & 37.1 & -5.3 & -2.6 & -60.2 \\
\hline-65 & 35.0 & 36.6 & -5.8 & -3.1 & -60.7 \\
\hline-60 & 34.6 & 36.1 & -6.3 & -3.6 & -61.1 \\
\hline-55 & 34.2 & 35.6 & -6.7 & -4.1 & -61.5 \\
\hline-50 & 33.8 & 35.1 & -7.2 & -4.6 & -61.9 \\
\hline-45 & 33.4 & 34.6 & -7.7 & -5.1 & -62.3 \\
\hline-40 & 33.0 & 34.1 & -8.2 & -5.6 & -62.8 \\
\hline-35 & 32.6 & 33.6 & -8.7 & -6.1 & -63.2 \\
\hline-30 & 32.2 & 33.1 & -9.2 & -6.6 & -63.6 \\
\hline-25 & 31.8 & 32.7 & -9.6 & -7.0 & -64.0 \\
\hline-20 & 31.4 & 32.2 & -10.1 & -7.5 & -64.4 \\
\hline-15 & 31.0 & 31.7 & -10.6 & -8.0 & -64.9 \\
\hline-10 & 30.6 & 31.2 & -11.1 & -8.5 & -65.3 \\
\hline-5 & 30.2 & 30.7 & -11.5 & -9.0 & -65.7 \\
\hline 0 & 29.8 & 30.2 & -12.0 & -9.5 & -66.1 \\
\hline 5 & 29.4 & 29.8 & -12.5 & -9.9 & -66.5 \\
\hline 10 & 29.0 & 29.3 & -12.9 & -10.4 & -66.9 \\
\hline 15 & 28.6 & 28.8 & -13.4 & -10.9 & -67.3 \\
\hline 20 & 28.2 & 28.3 & -13.9 & -11.4 & -67.7 \\
\hline 25 & 27.8 & 27.9 & -14.3 & -11.8 & -68.1 \\
\hline
\end{tabular}




\section{Free Reaction enthalpies for the reaction of $2 \mathrm{M} 1 \cdot \mathrm{LiCl}$}

A-Table 28. Free Reaction enthalpies for the reaction $2 \mathrm{M1} \cdot \mathrm{LiCl}$ in $\mathrm{kJ} / \mathrm{mol}$.

\begin{tabular}{|c|c|c|c|c|}
\hline$T\left[{ }^{\circ} \mathrm{C}\right]$ & CUBE A & CUBE B & CUBE C & CUBE D \\
\hline-90 & 42.0 & 51.7 & 82.8 & 31.3 \\
\hline-85 & 41.4 & 51.2 & 80.7 & 32.4 \\
\hline-80 & 40.9 & 50.7 & 78.6 & 33.4 \\
\hline-75 & 40.4 & 50.1 & 76.6 & 34.4 \\
\hline-70 & 39.8 & 49.6 & 74.5 & 35.5 \\
\hline-65 & 39.3 & 49.1 & 72.4 & 36.5 \\
\hline-60 & 38.8 & 48.6 & 70.4 & 37.5 \\
\hline-55 & 38.3 & 48.0 & 68.3 & 38.5 \\
\hline-50 & 37.7 & 47.5 & 66.3 & 39.6 \\
\hline-45 & 37.2 & 47.0 & 64.2 & 40.6 \\
\hline-40 & 36.7 & 46.5 & 62.2 & 41.6 \\
\hline-35 & 36.1 & 45.9 & 60.1 & 42.6 \\
\hline-30 & 35.6 & 45.4 & 58.1 & 43.6 \\
\hline-25 & 35.1 & 44.9 & 56.1 & 44.6 \\
\hline-20 & 34.5 & 44.4 & 54.0 & 45.7 \\
\hline-15 & 34.0 & 43.9 & 52.0 & 46.7 \\
\hline-10 & 33.5 & 43.4 & 50.0 & 47.7 \\
\hline-5 & 32.9 & 42.8 & 48.0 & 48.7 \\
\hline 0 & 32.4 & 42.3 & 45.9 & 49.7 \\
\hline 5 & 31.9 & 41.8 & 43.9 & 50.7 \\
\hline 10 & 31.4 & 41.3 & 41.9 & 51.7 \\
\hline 15 & 30.8 & 40.8 & 39.9 & 52.7 \\
\hline 20 & 30.3 & 40.3 & 37.9 & 53.7 \\
\hline 25 & 29.8 & 39.8 & 35.9 & 54.7 \\
\hline
\end{tabular}


A-Table 29. Free Reaction enthalpies for the reaction of $2 \mathrm{M1} \cdot \mathrm{LiCl}$ in $\mathrm{kJ} / \mathrm{mol}$.

\begin{tabular}{|c|c|c|c|c|c|}
\hline$T\left[{ }^{\circ} \mathrm{C}\right]$ & $\begin{array}{l}\mathrm{LiCl} \cdot \mathrm{D} 2 \cdot \mathrm{LiCl}- \\
\text { boat }\end{array}$ & $\begin{array}{l}\mathrm{LiCl} \cdot \mathrm{D} 2 \cdot \mathrm{LiCl}- \\
\quad \text { ladder }\end{array}$ & $\begin{array}{c}M 1 \cdot(\mathrm{LiCl}) 2 \cdot \mathrm{M} 1- \\
\text { boat }\end{array}$ & $\begin{array}{c}M 1 \cdot(\mathrm{LiCl}) 2 \cdot M 1- \\
\text { ladder }\end{array}$ & $\mathrm{LiCl} \cdot \mathrm{D} 1 \cdot \mathrm{LiCl}$ \\
\hline-90 & 57.7 & 59.7 & 17.3 & 19.9 & -37.9 \\
\hline-85 & 57.2 & 59.1 & 16.7 & 19.3 & -38.5 \\
\hline-80 & 56.7 & 58.5 & 16.1 & 18.7 & -39.0 \\
\hline-75 & 56.2 & 57.9 & 15.5 & 18.1 & -39.5 \\
\hline-70 & 55.7 & 57.3 & 14.9 & 17.6 & -40.0 \\
\hline-65 & 55.1 & 56.7 & 14.3 & 17.0 & -40.6 \\
\hline-60 & 54.6 & 56.1 & 13.7 & 16.4 & -41.1 \\
\hline-55 & 54.1 & 55.5 & 13.2 & 15.8 & -41.6 \\
\hline-50 & 53.6 & 54.9 & 12.6 & 15.2 & -42.1 \\
\hline-45 & 53.1 & 54.3 & 12.0 & 14.6 & -42.6 \\
\hline-40 & 52.6 & 53.7 & 11.4 & 14.0 & -43.2 \\
\hline-35 & 52.1 & 53.1 & 10.8 & 13.4 & -43.7 \\
\hline-30 & 51.6 & 52.6 & 10.3 & 12.8 & -44.2 \\
\hline-25 & 51.1 & 52.0 & 9.7 & 12.3 & -44.7 \\
\hline-20 & 50.6 & 51.4 & 9.1 & 11.7 & -45.2 \\
\hline-15 & 50.1 & 50.8 & 8.5 & 11.1 & -45.8 \\
\hline-10 & 49.6 & 50.2 & 7.9 & 10.5 & -46.3 \\
\hline-5 & 49.1 & 49.6 & 7.4 & 9.9 & -46.8 \\
\hline 0 & 48.6 & 49.0 & 6.8 & 9.3 & -47.3 \\
\hline 5 & 48.1 & 48.5 & 6.2 & 8.7 & -47.8 \\
\hline 10 & 47.6 & 47.9 & 5.6 & 8.2 & -48.3 \\
\hline 15 & 47.1 & 47.3 & 5.1 & 7.6 & -48.9 \\
\hline 20 & 46.6 & 46.7 & 4.5 & 7.0 & -49.4 \\
\hline 25 & 46.1 & 46.1 & 3.9 & 6.4 & -49.9 \\
\hline
\end{tabular}




\section{${ }^{1} \mathrm{H}$-DOSY-ECC-MW-determination parameters of TMPMgCl-LiCl 10 at $25^{\circ} \mathrm{C}$ to $-75^{\circ} \mathrm{C}$}

A-Table 30. ${ }^{1} \mathrm{H}$-DOSY-ECC-MW-determination of TMPMgCl-LiCl 10 in THF- $d_{8}(20 \mathrm{mM})$. PhN was used as internal reference in equimolar ratio with $\log D_{\text {ref, fix }}(\mathrm{PhN})=\mathbf{- 8 . 8 8 1 2}$.

\begin{tabular}{|c|c|c|c|c|}
\hline \multirow[t]{2}{*}{${ }^{1} \mathrm{H}$ DOSY } & & & & $25^{\circ} \mathrm{C}$ \\
\hline & & Species & $M W_{\text {calc }}[\mathrm{g} / \mathrm{mol}]$ & $M W_{\text {err }}[\%]$ \\
\hline$D_{\mathrm{x}}$ & $9.3030 \mathrm{E}-10$ & $1 \mathrm{~A}$ & 773 & 49 \\
\hline $\log D_{\mathrm{x}}$ & -9.0314 & $1 \mathrm{~B}$ & 544 & 28 \\
\hline $\log D_{\mathrm{x}, \text { norm }}$ & -9.0476 & $1 \mathrm{C}$ & 459 & 14 \\
\hline$D_{\text {ref }}(\mathrm{PhN})$ & $1.3647 \mathrm{E}-09$ & $1 C^{*}$ & 387 & -2 \\
\hline $\log D_{\text {ref }}(\mathrm{PhN})$ & -8.8650 & $1 \mathrm{D}$ & 344 & -14 \\
\hline \multirow[t]{3}{*}{$M W_{\text {det }}[\mathrm{g} / \mathrm{mol}]$} & 394 & $1 \mathrm{E}$ & 308 & -28 \\
\hline & & & & $+0^{\circ} \mathrm{C}$ \\
\hline & & Species & $M W_{\text {calc }}[\mathrm{g} / \mathrm{mol}]$ & $M W_{\text {err }}[\%]$ \\
\hline$D_{\mathrm{x}}$ & $6.7670 \mathrm{E}-10$ & $1 \mathrm{~A}$ & 773 & 50 \\
\hline $\log D_{\mathrm{x}}$ & -9.1696 & $1 \mathrm{~B}$ & 544 & 29 \\
\hline $\log D_{\mathrm{x}, \text { norm }}$ & -9.0418 & $1 \mathrm{C}$ & 459 & 16 \\
\hline$D_{\text {ref }}(\mathrm{PhN})$ & $9.7947 \mathrm{E}-10$ & $1 C^{*}$ & 387 & 0 \\
\hline $\log D_{\text {ref }}(\mathrm{PhN})$ & -9.0090 & $1 \mathrm{D}$ & 344 & -12 \\
\hline \multirow[t]{3}{*}{$M W_{\text {det }}[\mathrm{g} / \mathrm{mol}]$} & 385 & $1 \mathrm{E}$ & 308 & -25 \\
\hline & & & & $-25^{\circ} \mathrm{C}$ \\
\hline & & Species & $M W_{\text {calc }}[\mathrm{g} / \mathrm{mol}]$ & $M W_{\text {err }}[\%]$ \\
\hline$D_{\mathrm{x}}$ & $3.8980 \mathrm{E}-10$ & $1 \mathrm{~A}$ & 773 & 48 \\
\hline $\log D_{\mathrm{x}}$ & -9.4092 & $1 \mathrm{~B}$ & 544 & 26 \\
\hline $\log D_{\mathrm{x}, \text { norm }}$ & -9.0533 & $1 \mathrm{C}$ & 459 & 12 \\
\hline$D_{\text {ref }}(\mathrm{PhN})$ & $5.7933 \mathrm{E}-10$ & $1 C^{*}$ & 387 & -4 \\
\hline $\log D_{\text {ref }}(\mathrm{PhN})$ & -9.2371 & $1 \mathrm{D}$ & 344 & -17 \\
\hline \multirow[t]{3}{*}{$M W_{\text {det }}[\mathrm{g} / \mathrm{mol}]$} & 403 & $1 \mathrm{E}$ & 308 & -31 \\
\hline & & & & $-50^{\circ} \mathrm{C}$ \\
\hline & & Species & $M W_{\text {calc }}[\mathrm{g} / \mathrm{mol}]$ & $M W_{\mathrm{err}}[\%]$ \\
\hline$D_{\mathrm{x}}$ & $2.1200 \mathrm{E}-10$ & $1 \mathrm{~A}$ & 773 & 47 \\
\hline $\log D_{\mathrm{x}}$ & -9.6737 & $1 \mathrm{~B}$ & 544 & 24 \\
\hline $\log D_{\mathrm{x}, \text { norm }}$ & -9.0595 & $1 \mathrm{C}$ & 459 & 10 \\
\hline$D_{\text {ref }}(\mathrm{PhN})$ & $3.1960 \mathrm{E}-10$ & $1 C^{*}$ & 387 & -7 \\
\hline $\log D_{\text {ref }}(\mathrm{PhN})$ & -9.4954 & $1 \mathrm{D}$ & 344 & -20 \\
\hline$M W_{\text {det }}[\mathrm{g} / \mathrm{mol}]$ & 413 & $1 \mathrm{E}$ & 308 & -34 \\
\hline
\end{tabular}




\begin{tabular}{lcccc}
\hline & & & & $-75^{\circ} \mathrm{C}$ \\
& & Species & $M W_{\text {calc }}[\mathrm{g} / \mathrm{mol}]$ & $M W_{\text {err }}[\%]$ \\
$D_{\mathrm{x}}$ & $1.1030 \mathrm{E}-10$ & $1 \mathrm{~A}$ & 773 & 46 \\
$\log D_{\mathrm{x}}$ & -9.9574 & $1 \mathrm{~B}$ & 544 & 23 \\
$\log D_{\mathrm{x}, \mathrm{norm}}$ & -9.0625 & $1 \mathrm{C}$ & 459 & 9 \\
$D_{\text {ref }}(\mathrm{PhN})$ & $1.6743 \mathrm{E}-10$ & $1 \mathrm{C}^{*}$ & 387 & -8 \\
$\log D_{\text {ref }}(\mathrm{PhN})$ & -9.7762 & $1 \mathrm{D}$ & 344 & -21 \\
$M W_{\text {det }}[\mathrm{g} / \mathrm{mol}]$ & 418 & $1 \mathrm{E}$ & 308 & -36 \\
& & & & \\
\hline
\end{tabular}

\section{${ }^{7} \mathrm{Li}$-DOSY-ECC-MW-determination parameters of TMPMgCl-LiCl 10 at $25^{\circ} \mathrm{C}$ to $-75^{\circ} \mathrm{C}$}

A-Table 31. ${ }^{7} \mathrm{Li}$-DOSY-ECC-MW-determination of TMPMgCl-LiCl 10 in THF- $d_{8}(20 \mathrm{mM})$. PhN was used as internal reference in equimolar ratio with $\log D_{\text {ref, fix }}(\mathrm{PhN})=-\mathbf{8 . 8 8 1 2}$.

\begin{tabular}{|c|c|c|c|c|}
\hline \multirow[t]{2}{*}{${ }^{7} \mathrm{Li} \mathrm{DOSY}$} & & & & $25^{\circ} \mathrm{C}$ \\
\hline & & Species & $M W_{\text {calc }}[\mathrm{g} / \mathrm{mol}]$ & $M W_{\mathrm{err}}[\%]$ \\
\hline$D(\mathrm{Li})$ & $9.3330 \mathrm{E}-10$ & $1 \mathrm{~A}$ & 773 & 49 \\
\hline $\log D_{\mathrm{x}}(\mathrm{Li})$ & -9.0300 & $1 \mathrm{C}$ & 459 & 15 \\
\hline $\log D_{\mathrm{x}, \text { norm }}(\mathrm{Li})$ & -9.0462 & Li1 & 373 & -5 \\
\hline$D_{\text {ref }}(\mathrm{PhN})$ & $1.3647 \mathrm{E}-09$ & $1 C^{*}$ & 387 & -1 \\
\hline $\log D_{\text {ref }}(\mathrm{PhN})$ & -8.8650 & Li2 & 295 & -33 \\
\hline \multirow[t]{3}{*}{$M W_{\text {det }}$} & 392 & & & \\
\hline & & & & $+0^{\circ} \mathrm{C}$ \\
\hline & & Species & $M W_{\text {calc }}[\mathrm{g} / \mathrm{mol}]$ & $M W_{\text {err }}[\%]$ \\
\hline$D(\mathrm{Li})$ & $6.7490 \mathrm{E}-10$ & $1 \mathrm{~A}$ & 773 & 50 \\
\hline $\log D_{\mathrm{x}}(\mathrm{Li})$ & -9.1708 & $1 \mathrm{C}$ & 459 & 16 \\
\hline $\log D_{\mathrm{x}, \text { norm }}(\mathrm{Li})$ & -9.0430 & Li1 & 373 & -4 \\
\hline$D_{\text {ref }}(\mathrm{PhN})$ & $9.7947 \mathrm{E}-10$ & $1 C^{*}$ & 387 & 0 \\
\hline $\log D_{\text {ref }}(\mathrm{PhN})$ & -9.0090 & Li2 & 295 & -31 \\
\hline \multirow[t]{3}{*}{$M W_{\mathrm{det}}$} & 387 & & & \\
\hline & & & & $-25^{\circ} \mathrm{C}$ \\
\hline & & Species & $M W_{\text {calc }}[\mathrm{g} / \mathrm{mol}]$ & $M W_{\mathrm{err}}[\%]$ \\
\hline$D(\mathrm{Li})$ & $3.9330 \mathrm{E}-10$ & $1 \mathrm{~A}$ & 773 & 49 \\
\hline $\log D_{\mathrm{x}}(\mathrm{Li})$ & -9.4053 & $1 \mathrm{C}$ & 459 & 14 \\
\hline $\log D_{\mathrm{x}, \text { norm }}(\mathrm{Li})$ & -9.0494 & Li1 & 373 & -6 \\
\hline$D_{\text {ref }}(\mathrm{PhN})$ & $5.7933 \mathrm{E}-10$ & $1 C^{*}$ & 387 & -3 \\
\hline $\log D_{\text {ref }}(\mathrm{PhN})$ & -9.2371 & Li2 & 295 & -34 \\
\hline$M W_{\mathrm{det}}$ & 397 & & & \\
\hline
\end{tabular}




\begin{tabular}{|c|c|c|c|c|}
\hline & & & & $-50^{\circ} \mathrm{C}$ \\
\hline & & Species & $M W_{\text {calc }}[\mathrm{g} / \mathrm{mol}]$ & $M W_{\mathrm{err}}[\%]$ \\
\hline$D(\mathrm{Li})$ & $2.1940 \mathrm{E}-10$ & $1 \mathrm{~A}$ & 773 & 50 \\
\hline $\log D_{\mathrm{x}}(\mathrm{Li})$ & -9.6588 & $1 \mathrm{C}$ & 459 & 15 \\
\hline $\log D_{\mathrm{x}, \text { norm }}(\mathrm{Li})$ & -9.0446 & Li1 & 373 & -4 \\
\hline$D_{\text {ref }}(\mathrm{PhN})$ & $3.1960 \mathrm{E}-10$ & $1 C^{*}$ & 387 & -1 \\
\hline $\log D_{\text {ref }}(\mathrm{PhN})$ & -9.4954 & Li2 & 295 & -32 \\
\hline \multirow[t]{3}{*}{$M W_{\text {det }}$} & 389 & & & \\
\hline & & & & $-75^{\circ} \mathrm{C}$ \\
\hline & & Species & $M W_{\text {calc }}[\mathrm{g} / \mathrm{mol}]$ & $M W_{\text {err }}[\%]$ \\
\hline$D(\mathrm{Li})$ & $1.1840 \mathrm{E}-10$ & $1 \mathrm{~A}$ & 773 & 52 \\
\hline $\log D_{\mathrm{x}}(\mathrm{Li})$ & -9.9266 & $1 \mathrm{C}$ & 459 & 19 \\
\hline $\log D_{\mathrm{x}, \text { norm }}(\mathrm{Li})$ & -9.0317 & Li1 & 373 & 1 \\
\hline$D_{\text {ref }}(\mathrm{PhN})$ & $1.6743 \mathrm{E}-10$ & $1 C^{*}$ & 387 & 4 \\
\hline $\log D_{\text {ref }}(\mathrm{PhN})$ & -9.7762 & Li2 & 295 & -25 \\
\hline$M W_{\text {det }}$ & 370 & & & \\
\hline
\end{tabular}

\section{${ }^{1} \mathrm{H}$-DOSY-ECC-MW-determination parameters of TMP(H) at $25^{\circ} \mathrm{C}$ to $-75^{\circ} \mathrm{C}$}

A-Table 32. ${ }^{1} \mathrm{H}$-DOSY-ECC-MW-determination of the protonated amine TMP(H) in THF- $d_{8}(20 \mathrm{mM})$. $\mathrm{PhN}$ was used as internal reference in equimolar ratio with $\log D_{\text {ref,fix }}(\mathrm{PhN})=\mathbf{- 8 . 8 8 1 2}$. The $\mathrm{MW}$ of TMP(H), a highly spherical and compact molecule was calculated by using ECC $_{\mathrm{CS}}$.

\begin{tabular}{|c|c|c|c|c|}
\hline \multirow[t]{2}{*}{${ }^{1} \mathrm{H}$ DOSY } & & & & $25^{\circ} \mathrm{C}$ \\
\hline & & Species & $M W_{\text {calc }}[\mathrm{g} / \mathrm{mol}]$ & $M W_{\text {err }}[\%]$ \\
\hline$D(\operatorname{TMP}(\mathrm{H}))$ & $1.4980 \mathrm{E}-09$ & $\mathrm{TMP}(\mathrm{H})$ & 141 & -18 \\
\hline $\log D(\mathrm{TMP}(\mathrm{H}))$ & -8.824488187 & & & \\
\hline $\log D_{\mathrm{x}, \text { norm }}(\mathrm{TMP}(\mathrm{H}))$ & -8.8407 & & & \\
\hline$D_{\text {ref }}(\mathrm{PhN})$ & $1.3647 \mathrm{E}-09$ & & & \\
\hline $\log D_{\text {ref }}(\mathrm{PhN})$ & -8.8650 & & & \\
\hline \multirow[t]{3}{*}{$M W_{\text {det }}$} & 167 & & & \\
\hline & & & & $+0^{\circ} \mathrm{C}$ \\
\hline & & Species & $M W_{\text {calc }}[\mathrm{g} / \mathrm{mol}]$ & $M W_{\text {err }}[\%]$ \\
\hline$D(\operatorname{TMP}(\mathrm{H}))$ & $1.1400 \mathrm{E}-09$ & $\operatorname{TMP}(\mathrm{H})$ & 141 & -5 \\
\hline $\log D(\mathrm{TMP}(\mathrm{H}))$ & -8.943095149 & & & \\
\hline $\log D_{\mathrm{x}, \text { norm }}(\mathrm{TMP}(\mathrm{H}))$ & -8.8153 & & & \\
\hline$D_{\text {ref }}(\mathrm{PhN})$ & $9.7947 \mathrm{E}-10$ & & & \\
\hline $\log D_{\text {ref }}(\mathrm{PhN})$ & -9.0090 & & & \\
\hline$M W_{\text {det }}$ & 148 & & & \\
\hline
\end{tabular}




\begin{tabular}{|c|c|c|c|c|}
\hline & & & & $-25^{\circ} \mathrm{C}$ \\
\hline & & Species & $M W_{\text {calc }}[\mathrm{g} / \mathrm{mol}]$ & $M W_{\mathrm{err}}[\%]$ \\
\hline$D(\mathrm{TMP}(\mathrm{H}))$ & $6.7950 \mathrm{E}-10$ & $\mathrm{TMP}(\mathrm{H})$ & 141 & -3 \\
\hline $\log D(\mathrm{TMP}(\mathrm{H}))$ & -9.167810539 & & & \\
\hline $\log D_{\mathrm{x}, \text { norm }}(\mathrm{TMP}(\mathrm{H}))$ & -8.8119 & & & \\
\hline$D_{\text {ref }}(\mathrm{PhN})$ & $5.7933 \mathrm{E}-10$ & & & \\
\hline $\log D_{\text {ref }}(\mathrm{PhN})$ & -9.2371 & & & \\
\hline \multirow[t]{3}{*}{$M W_{\text {det }}$} & 146 & & & \\
\hline & & & & $-50^{\circ} \mathrm{C}$ \\
\hline & & Species & $M W_{\text {calc }}[\mathrm{g} / \mathrm{mol}]$ & $M W_{\text {err }}[\%]$ \\
\hline$D(\operatorname{TMP}(\mathrm{H}))$ & $3.8820 \mathrm{E}-10$ & $\mathrm{TMP}(\mathrm{H})$ & 141 & 4 \\
\hline $\log D(\mathrm{TMP}(\mathrm{H}))$ & -9.410944469 & & & \\
\hline $\log D_{\mathrm{x}, \text { norm }}(\mathrm{TMP}(\mathrm{H}))$ & -8.7968 & & & \\
\hline$D_{\text {ref }}(\mathrm{PhN})$ & $3.1960 \mathrm{E}-10$ & & & \\
\hline $\log D_{\text {ref }}(\mathrm{PhN})$ & -9.4954 & & & \\
\hline \multirow[t]{3}{*}{$M W_{\text {det }}$} & 136 & & & \\
\hline & & & & $-75^{\circ} \mathrm{C}$ \\
\hline & & Species & $M W_{\text {calc }}[\mathrm{g} / \mathrm{mol}]$ & $M W_{\mathrm{err}}[\%]$ \\
\hline$D(\mathrm{TMP}(\mathrm{H}))$ & $2.0622 \mathrm{E}-10$ & $\mathrm{TMP}(\mathrm{H})$ & 141 & 7 \\
\hline $\log D(\mathrm{TMP}(\mathrm{H}))$ & -9.685669217 & & & \\
\hline $\log D_{\mathrm{x}, \text { norm }}(\mathrm{TMP}(\mathrm{H}))$ & -8.7907 & & & \\
\hline$D_{\text {ref }}(\mathrm{PhN})$ & $1.6743 \mathrm{E}-10$ & & & \\
\hline $\log D_{\text {ref }}(\mathrm{PhN})$ & -9.7762 & & & \\
\hline$M W_{\text {det }}$ & 132 & & & \\
\hline
\end{tabular}


A-Table 33. Calculation of the molar van der Waals density $M D_{w}$ of all TMP species.

\begin{tabular}{lcccc}
\hline Species & Formula & $M W_{\text {calc }}$ & $\begin{array}{c}M D_{\mathrm{W}} \\
{\left[\mathrm{g} /\left(\mathrm{mol} \cdot \mathrm{m}^{3}\right)\right]}\end{array}$ & $\begin{array}{c}\Sigma V_{\mathrm{W}} \\
{\left[\mathrm{m}^{3}\right]}\end{array}$ \\
\hline & & & & \\
$\mathbf{1 A}$ & $\mathrm{C} 34 \mathrm{H} 68 \mathrm{Cl} 4 \mathrm{Li2Mg} 2 \mathrm{~N} 2 \mathrm{O} 4$ & 773 & $5.72 \mathrm{E}+29$ & $1.35 \mathrm{E}-27$ \\
$\mathbf{1 B}$ & $\mathrm{C} 26 \mathrm{H} 52 \mathrm{Cl} 2 \mathrm{Mg} 2 \mathrm{~N} 2 \mathrm{O} 2$ & 544 & $5.59 \mathrm{E}+29$ & $9.74 \mathrm{E}-28$ \\
$\mathbf{1 C}$ & $\mathrm{C} 21 \mathrm{H} 42 \mathrm{Cl} 2 \mathrm{LiMgNO} 3$ & 459 & $5.61 \mathrm{E}+29$ & $8.18 \mathrm{E}-28$ \\
$\mathbf{1 C}$ & $\mathrm{C} 17 \mathrm{H} 34 \mathrm{Cl} 2 \mathrm{LiMgNO} 2$ & 387 & $5.72 \mathrm{E}+29$ & $6.76 \mathrm{E}-28$ \\
$\mathbf{L i 1}$ & $\mathrm{C} 16 \mathrm{H} 32 \mathrm{Cl} 2 \mathrm{Li2O} 4$ & 373 & $5.64 \mathrm{E}+29$ & $6.62 \mathrm{E}-28$ \\
$\mathbf{1 D}$ & $\mathrm{C} 17 \mathrm{H} 34 \mathrm{ClMgNO} 2$ & 344 & $5.47 \mathrm{E}+29$ & $6.29 \mathrm{E}-28$ \\
$\mathbf{L i 2}$ & $\mathrm{C} 16 \mathrm{H} 32 \mathrm{LiO} 4$ & 295 & $4.99 \mathrm{E}+29$ & $5.92 \mathrm{E}-28$ \\
$\mathbf{1 E}$ & $\mathrm{C} 13 \mathrm{H} 26 \mathrm{Cl} 2 \mathrm{MgNO}$ & 308 & $6.05 \mathrm{E}+29$ & $5.09 \mathrm{E}-28$
\end{tabular}

In the DOSY-ECC-MW-determination of TMPMgCl-LiCl 10 no species with MWs lower than $308 \mathrm{~g} / \mathrm{mol}$ were observed, so aggregate $\mathbf{1 E}$ can be neglected. All other species work very well with the used ECCs. 


\section{REFERENCES}

[1] R. Neufeld, R. Michel, R. Herbst-Irmer, R. Schöne, D. Stalke, Chem. Eur. J. 2016, submitted.

[2] R. Neufeld, T. L. Teuteberg, R. Herbst-Irmer, R. A. Mata, D. Stalke, JACS 2016, accepted.

[3] R. Neufeld, D. Stalke, Chem. Eur. J. 2016, submitted.

[4] S. Bachmann, R. Neufeld, M. Dzemski, D. Stalke, Chem. Eur. J. 2016, accepted.

[5] R. Neufeld, M. John, D. Stalke, Angew. Chem. 2015, 127, 7100-7104; Angew. Chem. Int. Ed. 2015, 54, 6994-6998.

[6] R. Neufeld, D. Stalke, Chem. Sci. 2015, 6, 3354-3364

[7] R. Michel, T. Nack, R. Neufeld, J. M. Dieterich, R. A. Mata, D. Stalke, Angew. Chem. 2013, 125, 762-766; Angew. Chem. Int. Ed. 2013, 52, 734-738.

[8] a) E. Carl, D. Stalke, in Lithium Compounds in Organic Synthesis - From Fundamentals to Applications (Eds.: Renzo Luisi, V. Capriati), Wiley-VCH, Weinheim, 2014, pp. 1-31; b) T. Stey, D. Stalke, in The Chemistry of Organolithium Compounds (Eds.: Z. Rappoport, I. Marek), John Wiley \& Sons, New York, 2004, pp. 47-120.

[9] a) D. L. Comins, J. M. Nolan, I. D. Bori, Tetrahedron Lett. 2005, 46, 6697-6699; b) M. T. Reetz, in Encyclopedia of Reagents for Organic Synthesis, John Wiley \& Sons, Ltd, 2001; c) D. C. Bradley, I. M. Thomas, J. Chem. Soc. 1960, 3857-3861.

[10] R. E. Mulvey, S. D. Robertson, Angew. Chem. 2013, 125, 11682-11700; Angew. Chem. Int. Ed. 2013, 52, 11470-11487.

[11] a) R. E. Mulvey, Chem. Soc. Rev. 1991, 20, 167-209; b) D. R. Armstrong, D. Barr, W. Clegg, S. M. Hodgson, R. E. Mulvey, D. Reed, R. Snaith, D. S. Wright, J. Am. Chem. Soc. 1989, 111, 4719-4727; c) D. R. Armstrong, D. Barr, W. Clegg, R. E. Mulvey, D. Reed, R. Snaith, K. Wade, J. Chem. Soc., Chem. Comm. 1986, 869-870.

[12] a) Y. J. Kim, M. P. Bernstein, A. S. G. Roth, F. E. Romesberg, P. G. Williard, D. J. Fuller, A. T. Harrison, D. B. Collum, J. Org. Chem. 1991, 56, 4435-4439; b) E. Nakamura, K. Hashimoto, I. Kuwajima, Tetrahedron Lett. 1978, 19, 2079-2082.

[13] a) A.-C. Pöppler, M. Granitzka, R. Herbst-Irmer, Y.-S. Chen, B. B. Iversen, M. John, R. A. Mata, D. Stalke, Angew. Chem. 2014, 126, 13498-13503; Angew. Chem. Int. Ed. 2014, 53, 13282-13287; b) M. Granitzka, A.-C. Pöppler, E. K. Schwarze, D. Stern, T. Schulz, M. John, R. Herbst-Irmer, S. K. Pandey, D. Stalke, J. Am. Chem. Soc. 2012, 134, 1344-1351; c) D. B. Collum, A. J. McNeil, A. Ramirez, Angew. Chem. 2007, 17, 3060-3077; Angew. Chem. Int. Ed. 2007, 46, 3002-3017; d) J. F. Remenar, D. B. Collum, J. Am. Chem. Soc. 1998, 120, 4081-4086.

[14] a) J. L. Rutherford, D. B. Collum, J. Am. Chem. Soc. 2000, 123, 199-202; b) J. F. Remenar, B. L. Lucht, D. B. Collum, J. Am. Chem. Soc. 1997, 119, 5567-5572; c) F. E. Romesberg, D. B. Collum, J. Am. Chem. Soc. 1994, 116, 9198-9202.

[15] R. R. Fraser, M. Bresse, T. S. Mansour, J. Chem. Soc., Chem. Comm. 1983, 620-621.

[16] R. Li-Yuan Bao, R. Zhao, L. Shi, Chem. Commun. 2015, 51, 6884-6900.

[17] C. R. Hauser, H. G. Walker, J. Am. Chem. Soc. 1947, 69, 295-297.

[18] P. E. Eaton, C. H. Lee, Y. Xiong, J. Am. Chem. Soc. 1989, 111, 8016-8018.

[19] M. Shilai, Y. Kondo, T. Sakamoto, J. Chem. Soc. Perkin Trans. 1 2001, 442-444. 
[20] Y. Kondo, A. Yoshida, T. Sakamoto, J. Chem. Soc. Perkin Trans. 1 1996, 2331-2332.

[21] M. C. Whisler, S. MacNeil, V. Snieckus, P. Beak, Angew. Chem. 2004, 116, 2256-2276; Angew. Chem. Int. Ed. 2004, 43, 2206-2225.

[22] a) F. M. Piller, P. Appukkuttan, A. Gavryushin, M. Helm, P. Knochel, Angew.Chem. 2008, 120, 6907-6911; Angew. Chem. Int. Ed. 2008, 47, 6802-6806; b) J. P. Snyder, J. Am. Chem. Soc. 1995, 117, 11025-11026; c) T. Stemmler, J. E. Penner-Hahn, P. Knochel, J. Am. Chem. Soc. 1993, 115, 348-350; d) D. Stern, N. Finkelmeier, D. Stalke, Chem. Commun. 2011, 47, 2113-2115.

[23] A. Krasovskiy, V. Krasovskaya, P. Knochel, Angew. Chem. 2006, 118, 3024-3027; Angew. Chem. Int. Ed. 2006, 45, 2958-2961.

[24] a) D. Tilly, F. Chevallier, F. Mongin, P. C. Gros, Chem. Rev. 2014, 114, 1207-1257; b) T. Klatt, J. T. Markiewicz, C. Sämann, P. Knochel, J. Org. Chem. 2014, 79, 4253-4269; c) F. Mongin, A. Harrison-Marchand, Chem. Rev. 2013, 113, 7563-7727; d) G. C. Clososki, C. J. Rohbogner, P. Knochel, Angew. Chem. Int. Ed. 2007, 46, 7681-7684.

[25] J. Clayden, C. C. Stimson, M. Keenan, Chem. Commun. 2006, 1393-1394.

[26] T. Bresser, P. Knochel, Angew. Chem. 2011, 123, 1954 - 1958; Angew. Chem. Int. Ed. 2011, 50, 1914-1917.

[27] F. M. Piller, P. Knochel, Synthesis 2011, 2011, 1751-1758.

[28] D. R. Armstrong, P. García-Álvarez, A. R. Kennedy, R. E. Mulvey, J. A. Parkinson, Angew. Chem. 2010, 122, 3253-3256; Angew. Chem. Int. Ed. 2010, 49, 3185-3188.

[29] T. L. Rathman, J. A. Schwindeman, Org. Process Res. Dev. 2014, 18, 1192-1210.

[30] N. D. R. Barnett, R. E. Mulvey, W. Clegg, P. A. O'Neil, J. Am. Chem. Soc. 1991, 113, 8187-8188.

[31] Y. Ma, A. C. Hoepker, L. Gupta, M. F. Faggin, D. B. Collum, J. Am. Chem. Soc. 2010, 132, 1561015623.

[32] a) X. Sun, D. B. Collum, J. Am. Chem. Soc. 2000, 122, 2452-2458; b) J. F. Remenar, D. B. Collum, J. Am. Chem. Soc. 1997, 119, 5573-5582.

[33] M. F. Lappert, M. J. Slade, A. Singh, J. L. Atwood, R. D. Rogers, R. Shakir, J. Am. Chem. Soc. 1983, 105, 302-304.

[34] a) E. Hevia, A. R. Kennedy, R. E. Mulvey, D. L. Ramsay, S. D. Robertson, Chem. Eur. J. 2013, 19, 14069-14075; b) B. L. Lucht, D. B. Collum, J. Am. Chem. Soc. 1994, 116, 7949-7950.

[35] a) P. L. Hall, J. H. Gilchrist, A. T. Harrison, D. J. Fuller, D. B. Collum, J. Am. Chem. Soc. 1991, 113, 9575-9585; b) P. Renaud, M. A. Fox, J. Am. Chem. Soc. 1988, 110, 5702-5705.

[36] D. B. Collum, Acc. Chem. Res. 1993, 26, 227-234.

[37] F. E. Romesberg, J. H. Gilchrist, A. T. Harrison, D. J. Fuller, D. B. Collum, J. Am. Chem. Soc. 1991, 113, 5751-5757.

[38] a) J. F. Remenar, B. L. Lucht, D. Kruglyak, F. E. Romesberg, D. B. Collum, J. Org. Chem. 1997, 62, 5748-5754; b) F. E. Romesberg, D. B. Collum, J. Am. Chem. Soc. 1995, 117, 2166-2178; c) P. G. Williard, Q. Y. Liu, J. Am. Chem. Soc. 1993, 115, 3380-3381.

[39] a) R. D. Rogers, J. L. Atwood, R. Grüning, J. Organomet. Chem. 1978, 157, 229-237; b) D. Mootz, A. Zinnius, B. Böttcher, Angew. Chem. 1969, 81, 398-399; Angew. Chem. Int. Ed. 1969, 8, 378379.

[40] a) M. Driess, H. Pritzkow, M. Skipinski, U. Winkler, Organometallics 1997, 16, 5108-5112; b) J. Knizek, I. Krossing, H. Nöth, H. Schwenk, T. Seifert, Chem. Ber. 1997, 130, 1053-1062.

[41] R. Grüning, J. L. Atwood, J. Organomet. Chem. 1977, 137, 101-111. 
[42] a) K. F. Tesh, T. P. Hanusa, J. C. Huffman, Inorg. Chem. 1990, 29, 1584-1586; b) P. Williard, Acta Crystallogr. C 1988, 44, 270-272.

[43] S. Neander, U. Behrens, Z. Anorg. Allg. Chem. 1999, 625, 1429-1434.

[44] T. Fjeldberg, P. B. Hitchcock, M. F. Lappert, A. J. Thorne, J. Chem. Soc., Chem. Comm. 1984, 822824.

[45] J. C. Green, M. Payne, E. A. Seddon, R. A. Andersen, J. Chem. Soc., Dalton Trans. 1982, 887-892.

[46] a) G. C. Forbes, A. R. Kennedy, R. E. Mulvey, P. J. A. Rodger, R. B. Rowlings, J. Chem. Soc., Dalton Trans. 2001, 1477-1484; b) K. W. Henderson, P. G. Williard, Organometallics 1999, 18, 5620-5626; c) K. W. Henderson, A. E. Dorigo, Q.-Y. Liu, P. G. Williard, J. Am. Chem. Soc. 1997, 119, 11855-11863; d) H. Mack, G. Frenzen, M. Bendikov, M. S. Eisen, J. Organomet. Chem. 1997, $549,39-43$.

[47] a) C. Knapp, E. Lork, T. Borrmann, W.-D. Stohrer, R. Mews, Z. Anorg. Allg. Chem. 2005, 631, 1885-1892; b) G. C. Forbes, A. R. Kennedy, R. E. Mulvey, P. J. A. Rodger, Chem. Commun. 2001, 1400-1401; c) M. Karl, G. Seybert, W. Massa, K. Harms, S. Agarwal, R. Maleika, W. Stelter, A. Greiner, W. H. B. Neumüller, K. Dehnicke, Z. Anorg. Allg. Chem. 1999, 625, 1301-1309; d) F. T. Edelmann, F. Pauer, M. Wedler, D. Stalke, Inorg. Chem. 1992, 31, 4143-4146.

[48] a) M. S. Hill, G. Kociok-Kön, D. J. MacDougall, Inorg. Chem. 2011, 50, 5234-5241; b) P. L. Arnold, I. J. Casely, Z. R. Turner, R. Bellabarba, R. B. Tooze, Dalton Trans. 2009, 7236-7247.

[49] a) D. M. Cousins, M. G. Davidson, C. J. Frankis, D. Garcia-Vivo, M. F. Mahon, Dalton Trans. 2010, 39, 8278-8280; b) N. M. Clark, P. Garcia-Alvarez, A. R. Kennedy, C. T. O'Hara, G. M. Robertson, Chem. Commun. 2009, 5835-5837; c) P. P. Power, X. Xiaojie, J. Chem. Soc., Chem. Comm. 1984, 358-359.

[50] a) W. J. Evans, D. B. Rego, J. W. Ziller, Inorg. Chem. 2006, 45, 3437-3443; b) A. M. Domingos, G. M. Sheldrick, Acta Crystallogr. B 1974, 30, 517-519.

[51] B. Y. Kimura, T. L. Brown, J. Organomet. Chem. 1971, 26, 57-67.

[52] a) B. L. Lucht, D. B. Collum, Acc. Chem. Res. 1999, 32, 1035-1042; b) B. L. Lucht, M. P. Bernstein, J. F. Remenar, D. B. Collum, J. Am. Chem. Soc. 1996, 118, 10707-10718.

[53] P. G. Williard, M. A. Nichols, J. Am. Chem. Soc. 1991, 113, 9671-9673.

[54] a) P. Woidy, M. Buhl, F. Kraus, Dalton Trans. 2015, 44, 7332-7337; b) T. G. Müller, F. Karau, W. Schnick, F. Kraus, Angew. Chem. 2014, 126, 13913-13915; Angew. Chem. Int. Ed. 2014, 53, 1369513697; c) M. Neumeier, F. Fendt, S. Gärtner, C. Koch, T. Gärtner, N. Korber, R. M. Gschwind, Angew. Chem. 2013, 125, 4579-4582; Angew. Chem. Int. Ed. 2013, 52, 4483-4486; d) H. Nuss, M. Jansen, Angew. Chem. 2006, 118, 4476-4479; Angew. Chem. Int. Ed. 2006, 45, 4369-4371.

[55] a) J. Hey, D. M. Andrada, R. Michel, R. A. Mata, D. Stalke, Angew. Chem. 2013, 125, 1055510559; Angew. Chem. Int. Ed. 2013, 52, 10365-10369; b) R. Michel, R. Herbst-Irmer, D. Stalke, Organometallics 2010, 29, 6169-6171.

[56] P. Ji, J. Atherton, M. I. Page, J. Org. Chem. 2011, 76, 1425-1435.

[57] C. G. A. Inc., Handbook of compressed gases, Kluwer Academic Publishers Group, Dordrecht, Netherlands, 1999.

[58] A. F. Holleman, N. Wiberg, Lehrbuch der Anorganischen Chemie, Vol. 102, de Gruyter, Berlin, 2007.

[59] L. V. Coulter, J. R. Sinclair, A. G. Cole, G. C. Roper, J. Am. Chem. Soc. 1959, 81, 2986-2989.

[60] E. J. Behrman, J. Chem. Educ. 2006, 83, 1290.

[61] T. Francis, CRC Handbook of Chemistry and Physics, Vol. 87, Boca Raton, 2006. 
[62] a) T. M. Harris, C. M. Harris, J. Org. Chem. 1966, 31, 1032-1035; b) T. H. Vaughn, G. F. Hennion, R. R. Vogt, J. A. Nieuwland, J. Org. Chem. 1937, 02, 1-22; c) L. H. Baldinger, J. A. Nieuwland, J. Am. Chem. Soc. 1933, 55, 2851-2853.

[63] U. Wannagat, H. Niederprüm, Angew. Chem. 1959, 71, 574-574.

[64] J. F. Allan, W. Clegg, K. W. Henderson, L. Horsburgh, A. R. Kennedy, J. Organomet. Chem. 1998, 559, 173-179.

[65] W. Schlenk, W. Schlenk, Chem. Ber. 1929, 62, 920-924.

[66] F. W. Walker, E. C. Ashby, J. Am. Chem. Soc. 1969, 91, 3845-3850.

[67] a) Ö. Seven, M. Bolte, H.-W. Lerner, Acta Crystallogr. E 2013, 69, m424-m424; b) M. A. Nesbit, D. L. Gray, G. S. Girolami, Acta Crystallogr. E 2012, 68, m942; c) W. Ren, G. Zi, D.-C. Fang, M. D. Walter, Chem. Eur. J. 2011, 17, 12669-12682; d) A. G. Avent, C. F. Caro, P. B. Hitchcock, M. F. Lappert, Z. Li, X.-H. Wei, Dalton Trans. 2004, 1567-1577; e) A. L. Spek, P. Voorbergen, G. Schat, C. Blomberg, F. Bickelhaupt, J. Organomet. Chem. 1974, 77, 147-151; f) L. J. Guggenberger, R. E. Rundle, J. Am. Chem. Soc. 1968, 90, 5375-5378.

[68] a) E. C. Ashby, M. B. Smith, J. Am. Chem. Soc. 1964, 86, 4363-4370; b) E. C. Ashby, W. E. Becker, J. Am. Chem. Soc. 1963, 85, 118-119.

[69] M. B. Smith, W. E. Becker, Tetrahedron 1967, 23, 4215-4227.

[70] a) E. C. Ashby, J. Nackashi, G. E. Parris, J. Am. Chem. Soc. 1975, 97, 3162-3171; b) E. C. Ashby, G. E. Parris, J. Am. Chem. Soc. 1971, 93, 1206-1213; c) E. C. Ashby, S. Yu, J. Organometal. Chem. 1971, 29, 339-348.

[71] D. Seyferth, Organometallics 2009, 28, 1598-1605.

[72] P. García-Álvarez, D. V. Graham, E. Hevia, A. R. Kennedy, J. Klett, R. E. Mulvey, C. T. O'Hara, S. Weatherstone, Angew. Chem. 2008, 120, 8199-8201; Angew. Chem. Int. Ed. 2008, 47, 8079-8081.

[73] a) K.-C. Yang, C.-C. Chang, J.-Y. Huang, C.-C. Lin, G.-H. Lee, Y. Wang, M. Y. Chiang, J. Organomet. Chem. 2002, 648, 176-187; b) R. A. Bartlett, M. M. Olmstead, P. P. Power, Inorg. Chem. 1994, 33, 4800-4803.

[74] A. R. Kennedy, R. E. Mulvey, S. D. Robertson, Dalton Trans. 2010, 39, 9091-9099.

[75] A. S. Batsanov, P. D. Bolton, R. C. B. Copley, M. G. Davidson, J. A. K. Howard, C. Lustig, R. D. Price, J. Organomet. Chem. 1998, 550, 445-448.

[76] a) A. Krasovskiy, B. F. Straub, P. Knochel, Angew.Chem. 2006, 118, 165-169; Angew. Chem. Int. Ed. 2006, 45, 159-162; b) A. Krasovskiy, P. Knochel, Angew.Chem. 2004, 116, 3396-3399; Angew. Chem. Int. Ed. 2004, 43, 3333-3336.

[77] D. Li, I. Keresztes, R. Hopson, P. G. Williard, Acc. Chem. Res. 2008, 42, 270-280.

[78] a) E. O. Stejskal, J. E. Tanner, J. Chem. Phys. 1965, 42, 288-292; b) J. E. Tanner, Rev. Sci. Instrum. 1965, 36, 1086-1087; c) E. O. Stejskal, J. Chem. Phys. 1965, 43, 3597-3603.

[79] a) P. Pregosin, Acta Crystallogr. C 2013, 69, 1433-1436; b) K. A. Heisel, J. J. Goto, V. V. Krishnan, AJAC 2012, 3, 401-409; c) A. Macchioni, G. Ciancaleoni, C. Zuccaccia, D. Zuccaccia, Chem. Soc. Rev. 2008, 37, 479-489; d) P. S. Pregosin, P. G. A. Kumar, I. Fernández, Chem. Rev. 2005, 105, 2977-2998.

[80] a) I. V. Nesmelova, V. D. Fedotov, $B B A$ 1998, 1383, 311-316; b) D. Jayawickrama, S. Zink, D. V. Velde, R. I. Effiong, C. K. Larive, J. Biomol. Struct. Dyn. 1995, 13, 229-244. 
[81] a) S. Augé, P.-O. Schmit, C. A. Crutchfield, M. T. Islam, D. J. Harris, E. Durand, M. Clemancey, A.-A. Quoineaud, J.-M. Lancelin, Y. Prigent, F. Taulelle, M.-A. Delsuc, J. Phys. Chem., B. 2009, 113, 1914-1918; b) S. Viel, D. Capitani, L. Mannina, A. Segre, Biomacromolecules 2003, 4, 18431847; c) A. Chen, D. Wu, C. S. Johnson, J. Am. Chem. Soc. 1995, 117, 7965-7970.

[82] a) H. C. Gaede, K. Gawrisch, Magn. Reson. Chem. 2004, 42, 115-122; b) M. Braun, U. Hartnagel, E. Ravanelli, B. Schade, C. Böttcher, O. Vostrowsky, A. Hirsch, Eur. J. Org. Chem. 2004, 2004, 1983-2001; c) H. Ihm, J.-S. Ahn, M. S. Lah, Y. H. Ko, K. Paek, Org. Lett. 2004, 6, 3893-3896.

[83] a) S. C. Hunter, S.-J. Chen, C. A. Steren, M. G. Richmond, Z.-L. Xue, Organometallics 2015; b) M. M. Kireenko, E. A. Kuchuk, K. V. Zaitsev, V. A. Tafeenko, Y. F. Oprunenko, A. V. Churakov, E. K. Lermontova, G. S. Zaitseva, S. S. Karlov, Dalton Trans. 2015, 44, 11963-11976; c) E. Weisheim, C. G. Reuter, P. Heinrichs, Y. V. Vishnevskiy, A. Mix, B. Neumann, H.-G. Stammler, N. W. Mitzel, Chem. Eur. J. 2015, 21, 12436-12448; d) C. Su, R. Hopson, P. G. Williard, Eur. J. Inorg. Chem. 2013, 2013, 4136-4141; e) S. E. Baillie, W. Clegg, P. García-Álvarez, E. Hevia, A. R. Kennedy, J. Klett, L. Russo, Organometallics 2012, 31, 5131-5142.

[84] A. Einstein, R. Furth, A. D. Cowper, Investigations on the Theory of the Brownian Movement, Courier Dover Publications, Mineola, NY, 1956.

[85] E. L. Hahn, Phys. Rev. 1950, 80, 580-594.

[86] J. E. Tanner, J. Chem. Phys. 1970, 52, 2523-2526.

[87] D. E. Woessner, J. Chem. Phys. 1961, 34, 2057-2061.

[88] D. H. Wu, A. D. Chen, C. S. Johnson, J. Magn. Reson. 1995, 115, 260-264.

[89] S. J. Gibbs, C. S. Johnson Jr, Journal of Magnetic Resonance (1969) 1991, 93, 395-402.

[90] M. D. Pelta, G. A. Morris, M. J. Stchedroff, S. J. Hammond, Magn. Reson. Chem. 2002, 40, 147152.

[91] A. Jerschow, N. Müller, J. Magn. Reson. 1997, 125, 372-375.

[92] M. Nilsson, G. A. Morris, Chem. Commun. 2007, 933-935.

[93] K. F. Morris, C. S. Johnson, J. Am. Chem. Soc. 1992, 114, 3139-3141.

[94] M. Nilsson, A. M. Gil, I. Delgadillo, G. A. Morris, Chem. Commun. 2005, 1737-1739.

[95] B. Vitorge, D. Jeanneat, Anal. Chem. 2006, 78, 5601-5606.

[96] A. Jerschow, N. Müller, J. Magn. Reson. 1996, 123, 222-225.

[97] A. R. Waldeck, P. W. Kuchel, A. J. Lennon, B. E. Chapman, Prog. Nucl. Magn. Reson. Spectrosc. 1997, 30, 39-68.

[98] A. Spernol, K. Z. Wirtz, Z. Naturforsch., A Astrophys., Phys., Phys. Chem. 1953, 8, 522-532.

[99] H. C. Chen, S. H. Chen, J. Phys. Chem. 1984, 88, 5118-5121.

[100] F. Perrin, J. Phys. Radium 1936, 7, 1-11.

[101] R. Evans, Z. Deng, A. K. Rogerson, A. S. McLachlan, J. J. Richards, M. Nilsson, G. A. Morris, Angew. Chem. 2013, 125, 3281-3284; Angew. Chem. Int. Ed. 2013, 52, 3199-3202.

[102] D. K. Wilkins, S. B. Grimshaw, V. Receveur, C. M. Dobson, J. A. Jones, L. J. Smith, Biochemistry 1999, 38, 16424-16431.

[103] C. A. Crutchfield, D. J. Harris, J. Magn. Reson. 2007, 185, 179-182. 
[104] a) J. Guang, R. Hopson, P. G. Williard, J. Org. Chem. 2015, 80, 9102-9107; b) C. Su, R. Hopson, P. G. Williard, J. Am. Chem. Soc. 2013, 135, 14367-14379; c) G. Kagan, W. Li, R. Hopson, P. G. Williard, Org. Lett. 2009, 11, 4818-4821; d) D. Li, G. Kagan, R. Hopson, P. G. Williard, J. Am. Chem. Soc. 2009, 131, 5627-5634; e) D. Li, R. Hopson, W. Li, J. Liu, P. G. Williard, Org. Lett. 2008, 10, 909-911.

[105] a) A. J. Martinez-Martinez, D. R. Armstrong, B. Conway, B. J. Fleming, J. Klett, A. R. Kennedy, R. E. Mulvey, S. D. Robertson, C. T. O'Hara, Chem. Sci. 2014, 5, 771-781; b) D. R. Armstrong, A. R. Kennedy, R. E. Mulvey, S. D. Robertson, Dalton Trans. 2013, 42, 3704-3711; c) D. R. Armstrong, P. García-Álvarez, A. R. Kennedy, R. E. Mulvey, S. D. Robertson, Chem. Eur. J. 2011, 17, 67256730; d) P. García-Álvarez, R. E. Mulvey, J. A. Parkinson, Angew. Chem. 2011, 123, 9842-9845; Angew. Chem. Int. Ed. 2011, 50, 9668-9671.

[106] G. R. Fulmer, A. J. M. Miller, N. H. Sherden, H. E. Gottlieb, A. Nudelman, B. M. Stoltz, J. E. Bercaw, K. I. Goldberg, Organometallics 2010, 29, 2176-2179.

[107] S. Floquet, S. Brun, J.-F. Lemonnier, M. Henry, M.-A. Delsuc, Y. Prigent, E. Cadot, F. Taulelle, J. Am. Chem. Soc. 2009, 131, 17254-17259.

[108] M. A. Jacobson, I. Keresztes, P. G. Williard, J. Am. Chem. Soc. 2005, 127, 4965-4975.

[109] P. Paetzold, Chemie: Eine Einführung, Walter de Gruyter, Berlin, 2009.

[110] A. Bondi, J. Phys. Chem. 1964, 68, 441-451.

[111] The van der Waals radii were taken from: A. Bondi, J. Phys. Chem. 1964, 68, 441-451.

[112] D. Stern, N. Finkelmeier, K. Meindl, J. Henn, D. Stalke, Angew. Chem. 2010, 122, 7021-7024; Angew. Chem. Int. Ed. 2010, 49, 6869-6872.

[113] J. L. Rutherford, D. B. Collum, J. Am. Chem. Soc. 1999, 121, 10198-10202.

[114] P. Zhao, D. B. Collum, J. Am. Chem. Soc. 2003, 125, 4008-4009.

[115] R. E. Dinnebier, S. Neander, U. Behrens, F. Olbrich, Organometallics 1999, 18, 2915-2918.

[116] a) R. Michel, R. Herbst-Irmer, D. Stalke, Organometallics 2011, 30, 4379-4386; b) T. Kähler, U. Behrens, S. Neander, F. Olbrich, J. Organomet. Chem. 2002, 649, 50-54; c) R. E. Dinnebier, U. Behrens, F. Olbrich, Organometallics 1997, 16, 3855-3858.

[117] a) T. Nishinaga, D. Yamazaki, H. Stahr, A. Wakamiya, K. Komatsu, J. Am. Chem. Soc. 2003, 125, 7324-7335; b) Z. Hou, Y. Zhang, H. Tezuka, P. Xie, O. Tardif, T.-a. Koizumi, H. Yamazaki, Y. Wakatsuki, J. Am. Chem. Soc. 2000, 122, 10533-10543.

[118] R. R. Fraser, T. S. Mansour, S. Savard, J. Org. Chem. 1985, 50, 3232-3234.

[119] A.-C. Pöppler, M. M. Meinholz, H. Faßhuber, A. Lange, M. John, D. Stalke, Organometallics 2012, 31, 42-45.

[120] Y. Sarazin, S. J. Coles, D. L. Hughes, M. B. Hursthouse, M. Bochmann, Eur.J.Inorg.Chem. 2006, 3211-3220.

[121] Z. Rappoport, I. Marek, The Chemistry of Organomagnesium Compounds, Part I, John Wiley \& Sons Ltd, West Sussex, England, 2008.

[122] a) S. Grimme, J. Antony, S. Ehrlich, H. Krieg, J. Chem. Phys. 2010, 132, 154104; b) F. Weigend, R. Ahlrichs, Phys. Chem. Chem. Phys. 2005, 7, 3297-3305; c) A. D. Becke, J. Chem. Phys. 1993, 98, 5648-5652; d) A. D. Becke, Phys. Rev. A 1988, 38, 3098-3100; e) C. Lee, W. Yang, R. G. Parr, Phys. Rev. B 1988, 37, 785-789.

[123] A. Klamt, G. Schuurmann, J. Chem. Soc. Perkin Trans. 2 1993, 799-805. 
[124] a) D. Stern, M. Granitzka, T. Schulz, D. Stalke, Z. Naturforsch. 2010, 65b, 719-724; b) J. Tammiku-Taul, P. Burk, A. Tuulmets, J. Phys. Chem. A 2004, 108, 133-139.

[125] S. Sakamoto, T. Imamoto, K. Yamaguchi, Org. Lett. 2001, 3, 1793-1795.

[126] F. Blasberg, M. Bolte, M. Wagner, H.-W. Lerner, Organometallics 2012, 31, 1001-1005.

[127] a) A. V. Yakimansky, A. H. E. Müller, M. Van Beylen, Macromolecules 2000, 33, 5686-5692; b) W. Ming Keong, A. I. Popov, J. Inorg. Nucl. Chem. 1972, 34, 3615-3622; c) S. H. Bauer, T. Ino, R. F. Porter, J. Chem. Phys. 1960, 33, 685-691.

[128] C. Elschenbroich, F. Hensel, H. Hopf, Organometallchemie, Vol. 6, B. G. Teubner Verlag, Wiesbaden, 2008.

[129] a) J. Vollet, J. R. Hartig, K. Baranowska, H. Schnöckel, Organometallics 2006, 25, 2101-2103; b) U. Casellato, F. Ossola, Organometallics 1994, 13, 4105-4108.

[130] B. M. Gridley, T. J. Blundell, G. J. Moxey, W. Lewis, A. J. Blake, D. L. Kays, Chem. Commun. 2013, 49, 9752-9754.

[131] J. O. C. Jiménez-Halla, F. M. Bickelhaupt, M. Solà, J. Organomet. Chem. 2011, 696, 4104-4111.

[132] a) M. Brym, C. Jones, P. C. Junk, M. Kloth, Z. Anorg. Allg. Chem. 2006, 632, 1402-1404; b) S. Courtenay, P. Wei, D. W. Stephan, Can. J. Chem. 2003, 81, 1471-1476; c) F. Neumann, F. Hampel, P. v. R. Schleyer, Inorg. Chem. 1995, 34, 6553-6555.

[133] a) A. D. Sutton, T. Ngyuen, J. C. Fettinger, M. M. Olmstead, G. J. Long, P. P. Power, Inorg. Chem. 2007, 46, 4809-4814; b) A. S. Batsanov, M. G. Davidson, J. A. K. Howard, S. Lamb, C. Lustig, R. D. Price, Chem. Commun. 1997, 1211-1212; c) N. H. Buttrus, C. Eaborn, M. N. A. El-Kheli, P. B. Hitchcock, J. D. Smith, A. C. Sullivan, K. Tavakkoli, J. Chem. Soc., Dalton Trans. 1988, 381-391.

[134] D. Ardura, R. López, T. L. Sordo, J. Phys. Chem., B. 2005, 109, 23618-23623.

[135] a) J. Francos, B. J. Fleming, P. Garcia-Alvarez, A. R. Kennedy, K. Reilly, G. M. Robertson, S. D. Robertson, C. T. O'Hara, Dalton Trans. 2014, 43, 14424-14431; b) B. Baishya, J. Organomet. Chem. 2014, 769, 112-118.

[136] E. Hevia, A. R. Kennedy, R. E. Mulvey, S. Weatherstone, Angew.Chem. 2004, 116, 1741-1744; Angew. Chem. Int. Ed. 2004, 43, 1709-1712.

[137] R. Neufeld, T. L. Teuteberg, R. Herbst-Irmer, R. A. Mata, D. Stalke, JACS 2016, accepted.

[138] A. C. Jones, A. W. Sanders, M. J. Bevan, H. J. Reich, J. Am. Chem. Soc. 2007, 129, 3492-3493.

[139] W. Schlenk, J. Holtz, Ber. Dtsch. Chem. Ges. 1917, 50, 262-274.

[140] a) E. R. Johnson, A. D. Becke, J. Chem. Phys. 2006, 124, 174104; b) A. D. Becke, E. R. Johnson, J. Chem. Phys. 2005, 123, 154101; c) E. R. Johnson, A. D. Becke, J. Chem. Phys. 2005, 123, 024101.

[141] S. Grimme, Chem. Eur. J. 2012, 18, 9955-9964.

[142] F. Neese, WIREs Comput. Mol. Sci. 2012, 2, 73-78.

[143] a) D. Stalke, Chem. Soc. Rev. 1998, 27, 171-178; b) T. Kottke, D. Stalke, J. Appl. Crystallogr. 1993, 26, 615-619.

[144] T. Schulz, K. Meindl, D. Leusser, D. Stern, J. Graf, C. Michaelsen, M. Ruf, G. M. Sheldrick, D. Stalke, J. Appl. Crystallogr. 2009, 42, 885 - 891.

[145] SAINT v8.30c in Bruker APEX2 v2012.2, Bruker AXS Inst. Inc., Madison, USA, 2012.

[146] G. M. Sheldrick, SADABS-2015/1, Universität Göttingen, Germany, 2012.

[147] G. M. Sheldrick, Acta Crystallogr. 2015, A71, 3-8.

[148] G. M. Sheldrick, Acta Crystallogr. 2015, C71, 3-8. 
[149] a) A. Thorn, B. Dittrich, G. M. Sheldrick, Acta Cryst. 2012, A68, 448-451; b) L. Krause, R. Herbst-Irmer, G. M. Sheldrick, D. Stalke, J. Appl. Crystallogr. 2015, 48, 3-10; c) P. Müller, R. Herbst-Irmer, A. L. Spek, T. R. Schneider, M. R. Sawaya, Crystal Structure Refinement-A Crystallographer's Guide to SHELXL, Oxford University Press, Oxford (England), 2006.

[150] H. J. Reich, J. P. Borst, R. R. Dykstra, P. D. Green, J. Am. Chem. Soc. 1993, 115, 8728-8741.

[151] A. S. Galiano-Roth, D. B. Collum, J. Am. Chem. Soc. 1989, 111, 6772-6778. 


\section{Danksagung}

Viele Menschen haben zum Gelingen dieser Doktorarbeit beigetragen. Bei ihnen möchte ich mich hiermit nochmals bedanken:

An erster Stelle danke ich Herrn Prof. Dr. Dietmar Stalke für die Möglichkeit, bei ihm arbeiten und forschen zu dürfen und für die Freiheit, die er mir damit gelassen hat.

Herrn Prof. Dr. Sven Schneider danke ich für die Übernahme des Korreferats dieser Arbeit. Des Weiteren sei den Mitgliedern der Prüfungskommission gedankt.

Prof. Ricardo Mata und Thorsten Teuteberg danke ich für die wertvollen Ergebnisse aus theoretischen Rechnungen und für die tolle Zusammenarbeit.

Sebastian Bachmann, Thomas Niklas und Peter Stollberg danke ich für das Korrekturlesen dieser Arbeit. Ich danke euch ebenfalls für die vielen anregenden und teilweise auch sehr lustigen Diskussionen.

Dem gesamten Arbeitskreis danke ich für die angenehme Atmosphäre und die zahlreichen, leckeren Kaffee- und Kuchenpausen.

Den vielen „Diffraktometerschraubern“ und Martin Schlote sei ebenfalls vielmals für ihr Engagement und ihre Hilfestellung gedankt.

Michael John danke ich für die Beratung rund um das Thema NMR und Regine HerbstIrmer für die Unterstüzung rund um das Thema Kristallografie.

Ein weiterer Dank gilt Annegret Lehmberg, Benjamin Neding, Hendrik Alexander Schwenson und Sinja Pagel für die Herstellung der Hauser Base im Rahmen des MaWiPraktikums.

Des Weiteren danke ich meinen Forschungspraktikanten Markus Kinauer, Mona Wilken und Martin Dzemski für die unterstützende Hand bei der Forschungsarbeit. 
Der größte Dank gilt meiner Frau Nadine. Ohne deine Unterstützung und deinen Rückhalt hätte ich mich während meiner Promotion niemals so entfalten können. Ich bin sehr froh, dass ich dich habe.

Meinen Freunden danke ich vielmals für die Unterstützung und die schöne Ablenkung.

$\mathrm{Zu}$ guter Letzt danke ich meiner Familie. Ich danke euch für alles! 


\section{Curriculum Vitae}

\begin{tabular}{ll}
\hline Personal Data & \\
\hline Name: & Roman Neufeld \\
Date of birth: & 29.03.1985 in Pavlodar/Kazakhstan \\
Nationality: & German \\
Marital status: & Married to Nadine Wolf-Neufeld \\
Address: & Nelkenwinkel 5, 37081 Göttingen \\
E-mail: & rneufeld@uni.de
\end{tabular}

\section{Scientific Career}

09.2012-03.2016 Ph. D. thesis "DOSY External Calibration Curve Molecular Weight Determination as a Valuable Methodology in Characterizing Reactive Intermediates in Solution" in Prof. Dr. D. Stalke's workgroup at the Institute of Inorganic Chemistry at Georg-August-University Göttingen (Grade "summa cum laude")

09.2012-02.2016 Scientific researcher with Prof. Dr. D. Stalke within the GeorgAugust-University School of Science (GAUSS) doctoral programme with funding by Centre for Materials Crystallography (CMC) as part of the Danish National Research Foundation (DNRF93)

$10.2011-04.2012 \quad$ Master's thesis "Neuartige Alkalimetall-Amid-Ammoniakate" within Prof. Dr. D. Stalke's workgroup at the Institute of Inorganic Chemistry at Georg-August-University Göttingen (Grade 1.0)

10.2009-05.2012 Master of Science in Chemistry at Georg-August-University Göttingen (Grade 1.7)

04.2009-08.2009 Bachelor's thesis “Palladium-katalysierte Kupplungsreaktionen zur Synthese von C-Glycosiden" in Dr. D. B. Werz's workgroup at the Institute of Organic and Biomolecular Chemistry at Georg-AugustUniversity Göttingen (Grade 1.7)

10.2006-08.2009 Bachelor of Science in Chemistry at Georg-August-University Göttingen (Grade 2.3)

$10.2005-06.2006$ Guest student in Nanostructure Sciences at University of Kassel.

09.1997 - 06.2005 A-level at Gustav-Stresemann-Gymnasium, Bad Wildungen (Grade 2.4) 


\section{Experience in University and Abroad}

$09.2012-02.2016 \quad$ Scientific researcher with Prof. Dr. D. Stalke

$03.2013-02.2016$ Radiation protection commissioner within the group of Prof. Dr. D. Stalke

06.2015-02.2016 Supervision of practical labwork of major chemistry students (Inorganic Synthesis)

06.2014-06.2015 Supervision of practical labwork of material science students (Inorganic Material Synthesis)

09.2012-06.2014 Development of the chemisty labwork course Inorganic Material Synthesis for material science students.

$09.2007-06.2013$ IT service and network administrator at the Student Hostel ROKO, Göttingen

09.2011-06.2012 Supervision of practical labwork (incl. seminars) of medical and dental students (Chemistry for Medical and Dental Students)

04.2003 - 04.2005 Ice cream manufacturer at ice cream shop San Marco, Waldeck

Awards

07.2015 Winner of the award "Bester Vortrag" at Jung Chemiker Forum, Georg-August-University, Göttingen

06.2015

Second winner of the "Winterfeldt-Preis" at Leibniz University, Hannover

12.2014 Winner of the award "Preis für die beste nichtselbstständige Lehre am Institut für Anorganische Chemie” at Georg-August-University, Göttingen

10.2011

Winner of the "Paint Club Urban Art Battle Göttingen"

\section{Skills and Interests}

Languages German (native), English (fluent), Russian and French (basics)

EDV

Microsoft Office

Scientific data processing software (ChemDraw, Origin, Topspin, MestreNova)

Adobe Photoshop

Radiation protection Qualification: S1.1, S1.2, S1.3, S2.1, S2.2, S5, S6.1 (StrSchV) and R2.1 (RöV)

Expertise According to $\$ 5$ Chemikalienverbotsordnung

Hobbies Biking, football, table tennis, photography and image editing 


\section{Scientific Conference Participations}

1. Jung Chemiker Forum 2015, Göttingen, Germany-Talk “Akkurate Molekülgewichtsbestimmungen zur Charakterisierung reaktiver metallorganischer Verbindungen mittels einer neuen DOSY-NMR-Methode"

2. Winterfeldt-Preis 2015, Hanover, Germany-Talk (invited) "Charakterisierung reaktiver metallorganischer Verbindungen mittels einer neuen DOSY-NMR-Methode”

3. European Congress on Magnetic Resonance (EUROMAR) 2015, Prag, Czech RepublicPoster (Student Grant) “Accurate Molecular Weight Determination via DOSY-NMR”

4. 41st International Conference on Coordinaion Chemistry (ICCC-41) 2014, SingapurPoster "Alkali Metal Coordinated Hexamethyldisilazides from Liquid Ammonia"

5. Center for Materials Crystallography Conference (CMC) 2014, Göttingen, GermanyTalk "Structural Insight into Ammonia Solvated Alkalimetal-HMDS"

6. 20th EuCheMS Conference on Organometallic Chemistry (EuCOMC XX) 2013, St. Andrews, United Kingdom-Poster (Travel Grant) “Tune-up Aggregation of AlkaliHMDS with Ammonia"

7. Jung Chemiker Forum 2013, Göttingen, Germany-Poster “Ammonia, a Powerful Donorbase for Alkali Metal Hexamethyldisilazides"

\section{Scientific Publications in Print}

1. R. Neufeld, T. L. Teuteberg, R. Herbst-Irmer, R. A. Mata, D. Stalke "Solution Structures of Hauser Base ${ }^{i} \mathrm{Pr}_{2} \mathrm{NMgCl}$ and Turbo-Hauser Base ${ }^{i} \mathrm{Pr}_{2} \mathrm{NMgCl} \cdot \mathrm{LiCl}$ in THF and the Influence of LiCl on the Schlenk-Equilibrium" JACS 2016, accepted.

2. R. Neufeld, D. Stalke "Solution Structure of Turbo-Hauser Base TMPMgCl.LiCl in THF- $d_{8}$ " Chem. Eur. J. 2016, submitted.

3. R. Neufeld, R. Michel, R. Herbst-Irmer, R. Schöne, D. Stalke "Introducing a Hydrogen Bond Donor Into a Non Nucleophilic Brønsted Base: Structural insight into Ammonia Coordinated Alkali Metal Hexamethyldisilazide (HMDS, metal = Li, Na, K, Rb and Cs)" Chem. Eur. J. 2016, submitted.

4. S. Bachmann, R. Neufeld, M. Dzemski, D. Stalke "New External Calibration Curves (ECCs) for the Determination of Molecular Weights in Various Common NMR Solvents" Chem. Eur. J. 2016, accepted.

5. R. Neufeld, M. John, D. Stalke “The Donor-Base-Free Aggregation of Lithium Diisopropyl Amide in Hydrocarbons Revealed by a DOSY Method" Angew. Chem. Int. Ed. 2015, 54, 6994-6998; Angew. Chem. 2015, 127, 7100-7104, Frontispiece Cover. 


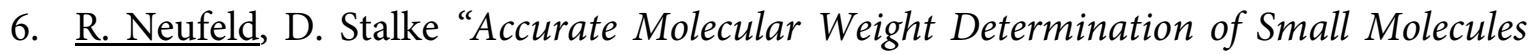
via DOSY-NMR by Using External Calibration Curves with Normalized Diffusion Coefficients" Chem. Sci. 2015, 6, 3354-3364.

7. P. P. Samuel, R. Neufeld, K. C. Mondal, H. W. Roesky, R. Herbst-Irmer, D. Stalke, S. Demeshko, F. Meyer, V. C. Rojisha, S. De, P. Parameswaran, A. C. Stuckl, W. Kaim, J. H. Christian, J. K. Bindra, N. S. Dalal "Cr(I)Cl as well as Cr+ are stabilised between two cyclic alkyl amino carbenes" Chem. Sci. 2015, 6, 3148-3153.

8. P. P. Samuel, K. C. Mondal, N. Amin Sk, H. W. Roesky, E. Carl, R. Neufeld, D. Stalke, S. Demeshko, F. Meyer, L. Ungur, L. F. Chibotaru, J. Christian, V. Ramachandran, J. van Tol, N. S. Dalal "Electronic Structure and Slow Magnetic Relaxation of LowCoordinate Cyclic Alkyl(amino) Carbene Stabilized Iron(I) Complexes” J. Am. Chem. Soc. 2014, 136, 11964-11971.

9. J. Wallbaum, R. Neufeld, D. Stalke, D. B. Werz "A Domino Approach to Dibenzopentafulvalenes by Quadruple Carbopalladation" Angew. Chem. Int. Ed. 2013, 52, 1324313246; Angew. Chem. 2013, 125, 13485-13488.

10. R. Michel, T. Nack, ․ Neufeld, J. M. Dieterich, R. A. Mata, D. Stalke "The Layered Structure of $\left[\mathrm{Na}(\mathrm{NH} 3)_{4}\right]\left[\right.$ Indenide] Containing a Square-Planar $\mathrm{Na}(\mathrm{NH} 3)_{4}{ }^{+}$Cation" Angew. Chem. Int. Ed. 2013, 52, 734-738; Angew. Chem. 2013, 125, 762-766.

11. D. C. Koester, M. Leibeling, R. Neufeld, D. B. Werz "A Pd-Catalyzed Approach to (1 $\rightarrow 6)$-Linked C-Glycosides” Org. Lett. 2010, 12, 3934-3937.

Göttingen, March 31, 2016 